\title{
Drifting 3D Underwater Wireless Sensor Networks for Pollution Monitoring
}

by

\author{
Yu Ren
}

A thesis

submitted to the Victoria University of Wellington in fulfilment of the requirements for the degree of

Doctor of Philosophy in Computer Science.

Victoria University of Wellington 



\begin{abstract}
Increasing demands by a growing population for food and oil have resulted in synchronous rises in the past 40 years of aquaculture and offshore oil drilling. Growth in these industries has highlighted the potential crippling impacts of coastal pollution, with marine farms likely to be susceptible to damage from harmful algal blooms and oil platforms to cause oil spills, as headlined by the 2010 Deepwater Horizon incident in the Gulf of Mexico.

Drifting underwater wireless sensor networks (UWSNs) represent a technology that can greatly enhance our understanding of such processes. Consisting of a swarm of untethered sensor nodes, an UWSN can be deployed over a large segment of the feature. The feature is carried through to different positions by underwater currents, the nodes, also drifting with the currents, are able to follow it and track it. To enable UWSNs, work is proceeding at multiple laboratories throughout the world on the design of localization, medium access control and routing protocols that can adapt to the problem that a drifting network topology has over short time intervals: frequent neighbour changes. However, the long-term impact that current drift has on 3D drifting UWSNs is poorly understood.

Current mobility models used to generate the motion of drifting nodes in simulation do not reflect how devices in real-life will disperse in water. At present, UWSN protocol schemes are evaluated in simulations where the current mobility of nodes is generated by unrealistic land-based random waypoint and other stochastic mobility models, or coarsely resolved numerical ocean models. Recently, a physically-inspired current mobility model known as MCM has been proposed. This is only defined along the water's surface, in 2D, however, and 3D extensions of this model have been simplistic and arbitrary.

The lack of realistic 3D current mobility models motivates this thesis to develop one so that the simulated evolution of UWSNs can more accurately reflect real-life. A consideration of the oceanographic data on which MCM is based is used to derive a 3D extension of the model that reflects observed features of the Gulf Stream current. The speed of the current declines with depth. This model is utilized to advect a drifting UWSN in an oil plume monitoring scenario and study the performance of
\end{abstract}


two pressure routing protocols, Depth Based Routing (DBR) and HydroCast, over time. Previously, these schemes had only been validated in unrealistic stochastic and depth-invariant mobility models and their time-averaged performances were only reported.

Our findings show that 3D UWSNs cannot expect to stay connected and functioning if nodes only passively drift with the currents, with results demonstrating that a fully connected network can be so dispersed after three hours that no paths to sinks exist at all. Nodes must be equipped with some form of mobility to prevent their being separated and carried out of the monitoring region. Autonomous underwater vehicles (AUVs) capable of omnidirectional motion are expensive however, and instead UWSNs consisting of profiling floats are considered in this thesis. Floats can move up and down by adjusting their buoyancy, which also allow them to move in 3D by exploiting water layers that are flowing in different directions to proceed along a desired heading. Recently, promising results in 2D path planning and formation control of floats have been presented. However, these works consider the floats to have infinite vertical velocity whereas in reality this is around 0.3 metres per second.

In this thesis, a practical node movement scheme is proposed for extending the coverage lifetime of a 3D UWSN consisting of floats. By accounting for the finite profiling velocity of floats, the scheme is able to position nodes at coverage holes with greater precision than existing 2D strategies. The scheme's performance is analysed by simulations. The results support the use of floats for achieving partial coverage, which can achieve similar levels of coverage as an AUV strategy while requiring less cost to deploy. In determining the lifetime of the network, we find that both energy and dispersion limit the lifetime of the network. Without propulsion, the nodes are carried out of and cease to cover the monitoring region. Using mobility to remain with the region, at the end of a 5 day mission duration floats have had to use up some or almost all of their battery capacity in profiling. 


\section{Acknowledgements}

First and foremost, I would like to thank Dr. Paul Teal, my supervisor. This work would never have been possible without your guidance, unending encouragement and above all faith in me.

I want to acknowledge the support of Victoria University of Wellington, the School of Engineering and Computer Science, and the Faculty of Graduate Research, in particular: Dr. Peter Andreae, Dr. Will Browne and Dr. Mark McGuinness for their helpful advice, Mark Davies for his grudging technical support, and Patricia Stein for her administrative acrobatics.

I would also like to use this opportunity to show my appreciation to my fellow labmates, notably Hang Yu, the two Craigs (Craig Watterson and Craig Anslow) and Muhammad Adeel Mahmood. It is only by having you lot as an outlet to gripe about our shared struggle that I have still have hair left to pull out.

There is another person that I want to thank, without whom I also would not have been able to complete this work, my good friend Charlie Hann. Seeing who can one-up the other, in writing, daring, scientific accomplishment and quoting Star Wars, is what gives me the motivation to do what I do.

And finally, I'd like to thank John Steinbeck, whose legacy and example have given me the world's best advice on technical writing: "the words that gather to my pen are honest, sturdy words, needing no adjectival crutches ... a close-reined, taut, economical English, unaccented and unlocalized. I put down no word that has not been judged for general understanding. Where my [audience] cannot fill in, I build up, and where my [audience] would be impatient with repetition, I cut. ... It is just as simple as that and I think it is the best prose I have ever written." 


\section{Acronyms}

ADCP Acoustic Doppler Current Profiler

ALACE Autonomous Lagrangian Circulation Explorer

ASV Autonomous Surface Vehicle

AUV Autonomous Underwater Vehicle

BER Bit Error Rate

BPSK Binary Phase Shift Keying

CTD Conductivity-Temperature-Depth

DBR Depth-Based Routing

DNR Dive aNd Rise

DVL Doppler Velocity Log

EM ElectroMagnetic

GPS Global Positioning System

HAB Harmful Algal Bloom

MCM Meandering Current Mobility model

MCM-SE Meandering Current Mobility with Surface Effects model

NIWA New Zealand's National Institute of Water and Atmospheric Research

NOAA The United States of America's National Oceanic and Atmospheric Administration

PDR Packet Delivery Ratio

QP Quadratic Program

RF Radio Frequency

RoI Region of Interest

ROMS Regional Ocean Modelling System 
STST Selective Tidal-Stream Transport

UUV Unmanned Underwater Vehicle

UWSN Underwater Wireless Sensor Network

VOCHA VOlume CHAnger

WOD World Ocean Database

WSN Wireless Sensor Network 


\section{Contents}

1 Introduction $\quad 1$

1.1 Problem statement . . . . . . . . . . . . . . . . . . . . 1

1.2 Motivations . . . . . . . . . . . . . . . . 6

1.3 Goals of this thesis . . . . . . . . . . . . . . . . . 10

1.4 Thesis contributions . . . . . . . . . . . . . . . . 11

1.5 Thesis structure . . . . . . . . . . . . . . . . . . . 12

2 Background $\quad 15$

2.1 The need for pollution monitoring . . . . . . . . . . . . . 15

2.1.1 Harmful algal blooms (HABs) . . . . . . . . . . . 15

2.1 .2 Oil spills . . . . . . . . . . . . . . . . . 17

2.2 The present state of underwater sensing . . . . . . . . . . . . 18

2.3 Drifting underwater wireless sensor networks . . . . . . . . . . 20

2.4 Motivating scenario: underwater oil plumes . . . . . . . . . . . . 21

3 A realistic 3D depth-decaying meandering current mobility model 23

3.1 Preliminaries . . . . . . . . . . . . . . . . . . . 27

3.2 The Meandering Current Mobility model (MCM) . . . . . . . . . . . 27

3.3 Previous 3D extensions of the MCM model . . . . . . . . . . . . . 35

3.4 The case for a depth-decaying current . . . . . . . . . . . . 37

3.5 The velocity profile of the current mobility model . . . . . . . . . . . 40

3.6 A 3D Gulf Stream model . . . . . . . . . . . . . . . . . . . . 45

3.7 A 3D coastal current model . . . . . . . . . . . . . . . . . 50

3.8 Summary . . . . . . . . . . . . . . . . . . . . . . 55

4 Performance of pressure routing in drifting 3D underwater sensor $\begin{array}{ll}\text { networks for oil plume monitoring } & 57\end{array}$

4.1 Pressure routing . . . . . . . . . . . . . . . . 58 
4.1.1 Depth-Based Routing (DBR) . . . . . . . . . . 61

4.1 .2 HydroCast . . . . . . . . . . . . . . . . . . . . . . . 62

4.2 Related work . . . . . . . . . . . . . . . . . 63

4.3 Simulation settings . . . . . . . . . . . . . . . 64

4.3 .1 Simulation scenario . . . . . . . . . . . . . . . . . 64

4.3 .2 Physical layer . . . . . . . . . . . . . . . . 65

4.3 .3 DBR ........................ 66

4.3 .4 HydroCast . . . . . . . . . . . . . . . . . 66

4.3 .5 Mobility . . . . . . . . . . . . . . 66

4.3 .6 Metrics ....................... 69

4.4 Performance results . . . . . . . . . . . . . . . . . 70

4.5 Summary . . . . . . . . . . . . . . . . . . . . 75

5 A practical coverage extension node movement scheme for float$\begin{array}{ll}\text { based UWSNs } & 77\end{array}$

5.1 Related work . . . . . . . . . . . . . . . . . 80

5.1 .1 Controllability results . . . . . . . . . . . . . 81

5.1.2 Well-behaved current-based schemes . . . . . . . . . 83

5.1.3 Selective Tidal-Stream Transport (STST)-based schemes . . . 87

5.1.4 General current schemes . . . . . . . . . . . . . . . 89

5.1.5 Current prediction-based schemes . . . . . . . . . . . 90

5.1 .6 In other fields . . . . . . . . . . . . . . . . . 91

5.1 .7 Summary . . . . . . . . . . . . . . . . . . . . 92

5.2 Novel movement scheme outline . . . . . . . . . . . . . . . . . . . 93

5.3 Conditions for 3D controllability . . . . . . . . . . . . . 96

5.3.1 A convex optimization problem . . . . . . . . . . . 99

5.4 Trajectory generation . . . . . . . . . . . . . . . . . . 102

5.4.1 Practical considerations . . . . . . . . . . . 106

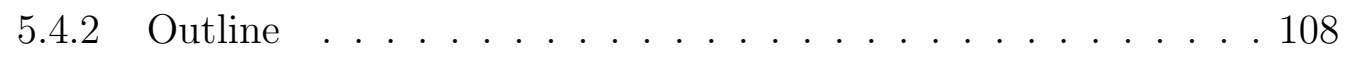

5.5 Target assignment . . . . . . . . . . . . . . . . . 109

5.6 Limitations . . . . . . . . . . . . . . . . . . . . . . . 111

5.7 Summary . . . . . . . . . . . . . . . . . . 111

6 Performance of a coverage extension node movement scheme 113

6.1 Simulation settings _ . . . . . . . . . . . . . . . . . 117

6.2 Evaluated schemes . . . . . . . . . . . . . . . . . . . . . 122

6.3 Metrics . . . . . . . . . . . . . . . . . . . . . . 127 
6.3 .1 Deployment cost . . . . . . . . . . . . . . . . . . . . . 129

6.3 .2 Coverage . . . . . . . . . . . . . . . . . . . . . . . . 129

6.3 .3 Cumulative coverage . . . . . . . . . . . . . . . 130

6.3 .4 Float energy consumption . . . . . . . . . . . . . . . . 131

6.3 .5 Distance travelled . . . . . . . . . . . . . . . . . . . . 132

6.3 .6 Hybrid AUV-float UWSNs . . . . . . . . . . . . . . . . . 132

6.3 .7 Partial AUV Coverage . . . . . . . . . . . . . . . . 133

6.4 Results . . . . . . . . . . . . . . . . . . . . . . . . 134

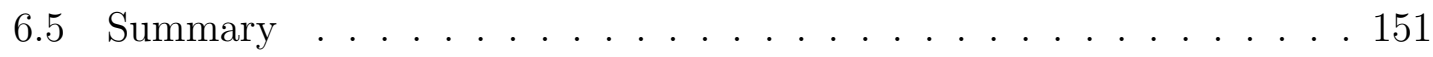

7 Conclusion and future research 153

7.1 Future work . . . . . . . . . . . . . . . . . 157

A The physical origins of ocean currents 163

A.1 Tidal and wave-induced currents . . . . . . . . . . . . . 163

A.2 Wind stress-induced currents . . . . . . . . . . . . . . . 163

A.3 Geostrophic currents . . . . . . . . . . . . . . . . 167

A.4 A depth-decaying mean current . . . . . . . . . . . 168

B Controllability analysis of Current 2 for floats 169

B.1 Controllability analysis of Current 2 in the static frame of reference . 170

B.2 Lagrangian tracking of a drifting RoI . . . . . . . . . 175

B.3 Equivalence of controllability problems in covering a drifting and static RoI . . . . . . . . . . . . . . . . . . . . . . . 179

B.4 Controllability analysis of Current 2 in the drifting frame of reference 186

$\begin{array}{ll}\text { C Network mobility simulator design } & 195\end{array}$

C.1 Motivation . . . . . . . . . . . . . . . . . . . . . . . . 195

C.2 Event simulation . . . . . . . . . . . . . . . . . . . 196

C.3 Scheme implementation . . . . . . . . . . . . . . . . . . . 197

C.4 Data logging . . . . . . . . . . . . . . . . . . . 198

D Velocity properties and energy consumption of ALACE floats 201

D.1 Model assumptions . . . . . . . . . . . . . . . . . . . . 201

D.2 Velocity limits . . . . . . . . . . . . . . . . . . . . 203

D.2.1 Forces acting on an ALACE profiling float . . . . . . . . 204

D.2.2 Terminal velocity . . . . . . . . . . . . . . . . . . 207

D.2.3 Velocity limits . . . . . . . . . . . . . . . . . . . . . 210 
D.3 Mobility electrical energy consumption . . . . . . . . . . . . . . 212

D.3.1 The energy cost of pumping . . . . . . . . . . . . . 212

D.3.2 Electrical energy usage . . . . . . . . . . . . . . . 214

$\begin{array}{ll}\text { E Supplementary mathematical results } & 217\end{array}$

E.1 Equivalence between Bower's current model and MCM . . . . . . . . 217

E.1.1 Physical significance of the MCM parameters . . . . . . . 219

E.2 Density profile computation . . . . . . . . . . . . . . . . 220 


\section{Chapter 1}

\section{Introduction}

\subsection{Problem statement}

Underwater wireless sensor networks (UWSNs) of untethered sensor nodes are a recent technology having the potential to transform how the ocean is observed. Current underwater sensing techniques are limited in the area they can observe at one time, whereas UWSNs are envisioned to enable large-scale, real-time, time-critical in-situ 3D aquatic monitoring. By drifting with the currents, they also allow features that disperse within the environment, such as pollution, to be tracked [41].

An UWSN, as shown in Figure 1.1, consists of a scalable ad-hoc network comprised of a large number, possibly hundreds, of low-cost wireless sensor nodes that are densely deployed to collaboratively monitor a region $[11,41,206]$. Each wireless sensor node is equipped with a number of sensors, a microprocessor and an acoustic modem. Power is supplied from an internal, typically battery-based, power supply, which has limited energy.

By deploying large numbers of nodes, UWSNs can provide sensing with high spatial and temporal resolution over a wide region. Unlike radio modems, whose transmissions are rapidly absorbed in water [34], acoustic modems enable nodes to communicate underwater and transmit data as soon as it is sensed. This allows for real-time monitoring, which is important in surveillance and environmental monitoring applications [11]. This is currently not possible with existing sensing methods where the nodes, for instance profiling floats, have to rise to the surface to transmit their sensed data via satellite or radio. The limited speed of underwater vehicles means that it can take several hours for the node to surface [1].

In UWSNs, data is transmitted to special sensor nodes known as sinks, which act as gateways. Underwater, the range of acoustic communications is low $[118,187]$. If 


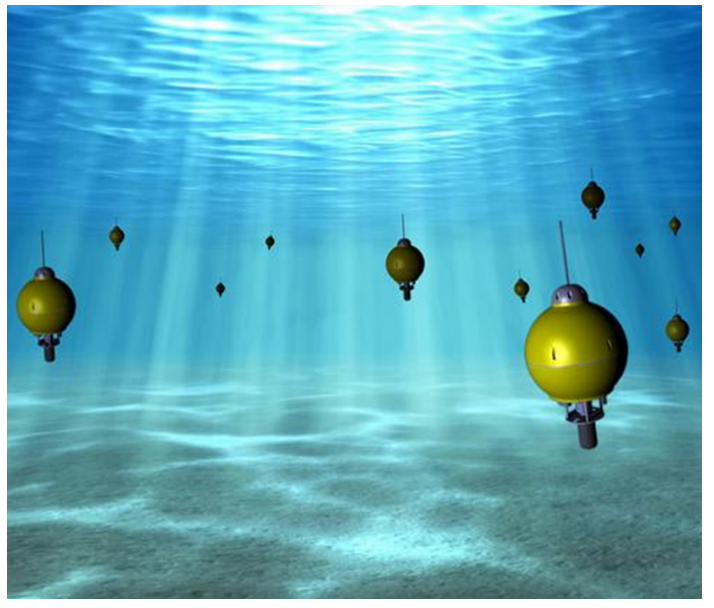

(a)

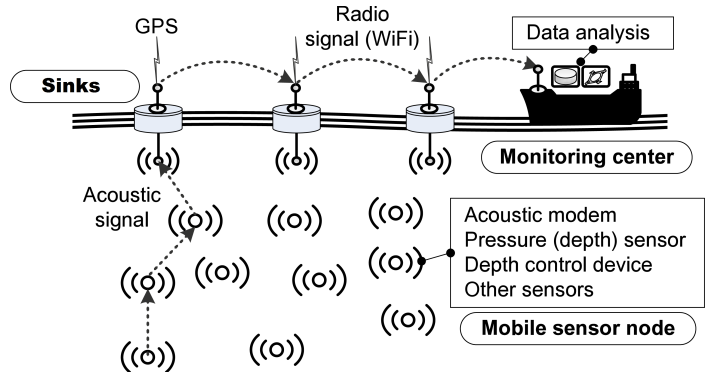

(b)

Figure 1.1: a) Drifting UWSN concept and b) architecture, consisting of wireless sensor nodes floating at different depths. Sensed data is transmitted via multi-hop routing to sink nodes. Images from [168] and [113].

a node is not within communications range of a sink, the data is sent via multi-hop routing. The data packet is transmitted to another node within range, that then transmits it to another neighbouring node until hopefully it is relayed to a sink. A packet can only reach a sink if a path actually exists. More formally, the network can be represented by a reachability graph, where the nodes of the graph are the sensors and an edge exists between any two sensors that are within communications range and can signal directly to each other [155]. A node is said to be connected if it can reach at least one sink by traversing the network's reachability graph.

UWSN nodes may be air-droppable, allowing them to be rapidly deployed to observe emerging events [112]. Once deployed, each device drifts passively with the currents. To keep the per-operation cost of nodes low, each device is equipped with an oil bladder to change its buoyancy, and hence depth. At the end of a mission, each node surfaces for ease of recovery. This significantly reduces the effective cost of the nodes as they can be reused, unlike sensors fixed to the seabed [41].

Since drifting UWSNs were first proposed as a concept in 2006 [41], they have yet to be deployed [58]. Before drifting UWSNs can be deployed, a number of challenges must be addressed, including the design of an entirely new protocol stack. On land, similar WSNs have been successfully put to use for multiple applications including sniper detection and industrial equipment monitoring [12], and protocols for these networks have been thoroughly evaluated [12]. The development of a practical UWSN is not simply a matter of directly adapting schemes from terrestrial WSNs however, 
as these protocols are unlikely to perform well due to the unique characteristics of the underwater environment [19].

UWSNs differ from terrestrial WSNs in two major respects. Nodes communicate using acoustic waves underwater, and are continuously subjected to movement from underwater currents.

Because of the skin effect induced by the high conductivity of seawater, electromagnetic $(\mathrm{EM})$ radio frequency $(\mathrm{RF})$ waves are rapidly absorbed within a few metres of propagation in water [34], making radio unsuitable for underwater communications. Acoustic communications are employed instead, with sound waves penetrating much further than RF waves. Data rates are still limited, especially at longer ranges. Acoustic waves experience absorption losses that increase with frequency. This limits the frequency range available for communications at feasible power levels to $0-100 \mathrm{kHz}$ for distances less than $100 \mathrm{~m}, 0-10 \mathrm{kHz}$ over $1-10 \mathrm{~km}$ and 0-1 kHz for distances between $10-100 \mathrm{~km}$ [187]. The low bandwidth results in low data rates, with the best research systems being able to achieve a rate-range product of $40 \mathrm{~km} \times \mathrm{kbps}$ [99], still roughly 100 times lower than between nodes on the surface, which can use RF waves to communicate [199]. Working ranges of commercial acoustic modems are often smaller, with an acoustic link data rate of $31.2 \mathrm{kbps}$ at $350 \mathrm{~m}$ being typical [118].

Node drift induces frequent changes in network topology. The speed of currents in the ocean is $\approx 0.1 \mathrm{~ms}^{-1}$ [95]. For a representative underwater communications range of $100-1000 \mathrm{~m}$ at $0.1 \mathrm{~ms}^{-1}$ nodes can potentially be displaced out of contact with another stationary sensor or node moving with the opposite velocity in the order of minutes.

These features of the underwater environment typically result in lowered performance when protocols for terrestrial WSNs are run on UWSNs. As a result the design of new schemes optimized specifically for underwater is now a major focus of UWSN research, including medium access control [175], localization [58, 190], routing [18] and synchronization protocols [189]. The impact these properties have on protocol design are illustrated below, employing routing and localization as examples.

The rapidity with which neighbours change due to current mobility means that route information quickly becomes invalidated. For this reason, routing protocols that rely on network-wide flooding to keep path information up-to-date, such as proactive routing protocols developed for terrestrial wireless sensor and ad-hoc networks, are considered unsuitable for UWSNs [18]. In proactive schemes, each node maintains a table with route information to every other device in the network. Whenever the 
neighbourhood around a node changes, this information is propagated throughout the network so that all sensors' tables are kept up-to-date. This design is viable and the messaging overhead manageable on land, as nodes are static. Hence, updates are rare and due mainly to infrequent node failures. In UWSNs, where the make-up of neighbourhoods is constantly changing due to mobility, frequent broadcasts are required. Due to the low bandwidth of communications underwater, these exchanges end up taking up a large proportion of the channel capacity, resulting in congestion and packet collisions.

Consequently, many of the routing protocols being developed for UWSNs limit the number and extent to which packets are transmitted, such as UnderWater Diffusion [112] and geographic routing protocols [113,134,200,204,205]. For instance, UnderWater Diffusion was found [112] to be able to achieve a packet delivery ratio above $90 \%$ compared to less than $30 \%$ for Direction Diffusion when each protocol was run separately in the same simulation scenario. Compared to UnderWater Diffusion that was designed to use no proactive routing message exchange and a negligible amount of on-demand floods, Direction Diffusion [85], a popular data-centric routing protocol, periodically needs to flood interests and exploratory messages to handle mobility, and induced more congestion and packet drops as a result. Nodes in geographic routing select potential forwarders for a packet based on the position of their neighbours, generally preferring devices that are closer to the destination. Nodes only require information on their neighbours, necessitating regular messaging of position updates within a 1- or 2-hop radius rather than expensive network-wide flooding in proactive and reactive protocols. Where destinations are limited to the surface the full 3D location of sensors may be unnecessary, and packets can be routed by forwarding to nodes that are higher up in depth. This is the premise of pressure routing protocols $[113,134,205]$, a subtype of underwater geographic routing protocols.

Terrestrial nodes can acquire their location with Global Position System (GPS) receivers [190], and this is the common solution on land. This is impossible for subsurface sensors as the $1.5 \mathrm{GHz}$ signals transmitted by satellites are only able to penetrate a few metres underwater [34]. GPS can still be employed by devices on the surface, and one way to localize underwater nodes is for these anchor nodes to transmit acoustically so that sensors within range of three or more anchors can trilaterate their position by the travel time of the signals. Once these sensors are localized, they can act as anchors and begin broadcasting signals. This process is then repeated, with nodes further and further hops distant from surface devices being 
localized. The messaging overhead, which must be ongoing as nodes are continually drifting, is high, and, along with routing schemes [38], the design of lightweight protocols [190], i.e. having low communications costs, remains an ongoing challenge.

Although mobility motivates the need for localization and new routing protocols, very little is known about how the network changes shape as it drifts with the currents. Proposed UWSN routing protocols have taken into account drifting nodes and simulated their motion. However, as seen earlier, the reasoning for existing routing protocols being unsuitable is that they need frequent updates due to the topology being dynamic and studies have focused on this aspect. Many works have been content with employing unrealistic stochastic land-based mobility models for the underwater current $[204,205]$ and simulating the networks for a short duration, from $200 \mathrm{~s}$ [204] to 1 hour [113]. These are sufficient to verify that the protocol is resilient to neighbour changes. No study of the state of a 3D drifting UWSN or performance of underwater routing protocols over a long period of time has been recorded, where by "long", a duration of a few hours or more is meant. In practice, present day survey missions with autonomous underwater vehicles (AUVs) last on the order of days [29], where a typical AUV has an endurance of 3 days [104], and the buoyancy driven devices employed in UWSNs have been deployed for a year at a time [160]. A UWSN should be able to provide similar duration monitoring capabilities. Yet prolonged observation of the network is needed, as current mobility causes the network to drift apart [32].

Nodes are only able to sense within a finite range. For instance a submarine can only be reliably detected within some distance of a hydrophone. To monitor all events occurring in a region of interest (RoI), the area to be monitored, nodes in an UWSN must be distributed throughout it so that each point inside the space is within sensing range of a device. The set of such points is called the coverage of the network [81]. Data sensed by a node is only useful if it can be transferred to a sink, so that the information can then be sent to an end user to be acted upon. This requires the node to be connected, meaning that there exists a communication path to a sink consisting of consecutive nodes that are within transmission and reception range of each other [65]. The definition of connected coverage, as the set of all points inside the RoI that is within sensing range of a connected node, follows naturally from these concepts. Connected coverage provides a useful measure of the liveliness or quality of service of a UWSN, being the area where events can be detected and outside users can be notified of said events [52]. The network is considered nonfunctional or not alive when a sufficient area can no longer be sensed. Currents negatively 
impact connectivity and coverage by causing nodes to drift away from the original positions they are covering and out of communications range of other devices. This requires the constant redeployment of devices via a node movement scheme so that they remain networked. The goal of such movement includes repairing coverage holes [207], areas that are no longer being monitored due to all nearby nodes having moved out of sensing range.

\subsection{Motivations}

Little attention has been paid to the impact that prolonged current drift has on UWSNs before researchers moved on to active development of UWSN protocols. However, anticipating how the network moves is a more fundamental issue, as it is needed to establish whether the drifting UWSN concept makes sense. Applications for floating UWSNs will be severely restricted if the network becomes disconnected after a few hours of drift. It also raises the question of what exactly is being sensed. As well as short-term time-critical aquatic exploration missions, drifting UWSNs were proposed for long-term non-time-critical monitoring of static regions, such as oil and gas fields [41]. In rivers or other directed flows, such as the Loop Current that runs through the (heavily exploited for oil) Gulf of Mexico, drifting nodes are expected to move out of a fixed region in one direction [109]. Even if the network is able to remain operational, with nodes routing packets to the surface, the data will no longer be of interest as it is not sensing the field.

Studies of how UWSNs drift are hampered by the fact that no adequate 3D current mobility model exists for inferring the inter-neighbour interactions among UWSN nodes. A mobility model specifies the velocities with which nodes travel at each time instant in a simulation; for floats used as drifting nodes this is the velocity of the current at each float's location [46]. A 2D network refers to a network where all nodes a deployed along a horizontal surface. A 2D mobility model defines velocities only along a horizontal surface. Terrestrial networks, which are placed along the ground, can be considered approximately 2D. UWSNs, where nodes may be suspended at different depths, are often 3D.

Currents have been defined in the literature by ocean forecast models and synthetic mobility models. Ocean forecast models, also known as numerical ocean models, consider the various forces and conservation laws affecting the ocean. These are formulated into governing equations for oceanic motions, a system of non-linear partial differential equations, that the model solves for the current velocity field [96, p. 28]. 
The current velocities are only solved at widely spaced grid points, however. The computational complexity of partial differential equation solvers increases rapidly with resolution, with a doubling of the resolution increasing the computation time of a 3D model sixteenfold [149, p. 180]. Thus, even using supercomputers and restricting the domain to a small ocean region, the resolution of typical present-day ocean forecast systems is around $1-10, \mathrm{~km}$ horizontally [96, p. 22] [37, 179]. This leads to the output of forecast systems being of limited use for UWSN simulations. These models are unable to reflect realistic neighbourhood mobility changes, important to connectivity and routing protocol performance. The spacing between neighbouring nodes in UWSNs, around $100 \mathrm{~m}$ as this is the transmission range of underwater acoustic modems, is an order of magnitude less than the model resolution [118,205]. The velocity of two neighbours inside a grid cell is found by interpolating the gridding currents, resulting in neighbour dynamics that are primarily influenced by the interpolation method chosen rather than the current. Present day use of numerical ocean models has been largely to study the mesoscale $(100 \mathrm{~km})$ mobility of eddies [137], drifters [182] and widely-spaced float arrays [42], where the distance travelled on a scale of days [42] is of interest rather than the fine $4 \mathrm{D}$ resolution needed for investigating UWSN behaviour.

Another problem with ocean forecast models is the accuracy of their subsurface current predictions. The forces and other inputs into the governing equations solved by the model need to be very accurately specified. Due to flows being typically chaotic and turbulent, any small deviation will influence the current prediction exponentially over time [141], resulting in a modelled ocean that rapidly diverges from the real ocean. Numerical ocean models retain their accuracy by continually inserting in observed ocean readings for the model parameters, in a process known as assimilation [96, p. 729]. This restricts accurate predictions to regions where such sensing infrastructure, for instance moorings, are in place. Furthermore, sensed ocean data is most readily available only for the surface via HF (High Frequency) radar and satellite data, and assimilative models such as ROMS (Regional Ocean Model System) have demonstrated poor prediction performance when modelling deep water currents where there is a large vertical complexity of the currents [179]. Past experiments using ROMS to anticipate the 3D trajectories of AUVs have resulted in significant differences between the predicted and actual trajectories. In Smith et al. [179], 29.4\% mean surfacing errors were reported. The need to have detailed knowledge of ocean conditions in the region, with which to initialize the model, also makes it difficult for the casual network researcher to set up and use such a program 
without making errors [32].

To represent the current motions, most UWSN studies have instead employed synthetic mobility models such as random waypoint [35, 204, 205] and random walk [36,119]. These models were originally developed for and have been widely utilized in the study of land-based ad hoc networks [30]. Thus, an advantage of their use is their existing implementation in network simulators like NS-2 [22, 204]. In these models, nodes move independently of each other, hence the other name for them: stochastic mobility models [32]. For instance, in random waypoint, nodes pick a random destination inside the deployment region to move to with random speed chosen uniformly between velocity limits. Upon reaching its destination, the node waits a set pause time before repeating the process [22]. This independent motion is not representative of current mobility, which is that of a flow given by a velocity field [37] and where the velocity is dependent on the position within the field. These models are rather intended for situations on land where autonomous agents are moving under their own power, such as the movement of people, and their mobile phones, about a room where the pause time denotes the restlessness of the people [92]. The restriction of nodes to the deployment region (with nodes being reflected off the boundaries of the region in the mobility model) also produces counterintuitive results. Untethered nodes dropped underwater will typically disperse and be carried far away from the box in which they were deployed. This observation is exploited to study currents in the ocean, with many oceanographic agencies running programs of dropping drifters in the form of plastic cards in a section of water to scatter and eventually wash ashore. These are tagged with the return address of the agency, so that a beachgoer discovering such a card can report it and the agency can infer ocean currents from where the drifter ended up [140, p. 117]. In models like random waypoint, nodes not only are restricted to the deployment region but, rather than drifting apart, will end up more clustered together than their initial uniform distribution, resulting in improved connectivity, lower hop count and end-to-end delay. This is because, when a snapshot of the network is taken, many nodes will be found passing through a central location on the way to their randomly generated locations [22].

Recently, a current mobility model for coastal currents has been proposed that its authors consider to be realistic [32]. In this Meandering Current Mobility model (MCM), the current is described by a function defining the current velocity at every point to arbitrary resolution. As a consequence of the current being defined in terms of its motion only, the model is known as being kinematic (rather than dynamical in 
the case where forces are considered). The authors' claim realism because kinematic models have being widely used in fluid dynamics and the model is based on actual current observations, although in Chapter 3, when the experimental data on which MCM is based is investigated further, it is found that MCM is unlikely to represent all coastal currents but only the Gulf Stream. Since being proposed in 2008, MCM has rapidly gained acceptance among the wireless underwater acoustic sensor network community, being used in studies of various routing protocols [113,134], and node movement [121] and localization schemes [59] to advect nodes in place of land-based stochastic models. MCM lacks the accuracy and coarsely resolved velocity issues limiting the applicability of stochastic and numerical ocean models respectively, resulting in the model being described as late as 2014 as "the only UWSN mobility model available in the literature" [123]. The existence of a realistic current mobility model in MCM does little to address the problem of a lack of realistic 3D current mobility models however, as MCM is only defined in 2D for the sea surface.

With the absence of current mobility models, the impact of current drift on network performance over time has only been studied realistically in 2D [32], and for 3D with terrestrial or naive mobility models [59,119]. After proposing the MCM model, Caruso et al. [32] study the dynamic coverage and connectivity over time of a 2D (deployed along the water's surface) drifting UWSN being advected by the current. The network is found to spread apart. Beginning with the network as being densely deployed in a small region, so that all nodes are connected, after 120 hours the area in which sensors are located has increased around $18000 \%$. Liu et al. [119] simulate a 3D drifting UWSN for 220 hours to see how many nodes remain within a static RoI and how much of the region remains covered. However, a simplistic random walk mobility model is employed for the current.

The lack of realistic mobility models is highlighted in separate surveys on underwater localization by Erol-Kantarci et al. [58] and Tan et al. [190], where both groups of authors identify open issues with the understanding of how current mobility affects localization performance. Erol-Kantarci et al. [58] find that more accurate mobility models are needed, while Tan et al. [190] note that the evaluation of localization protocols for networks in deep sea environments is hampered by the lack of suitable mobility models. 


\subsection{Goals of this thesis}

Ocean currents impact the data that can be communicated to and from a network, and mobility has not been investigated for physically-inspired 3D currents. This led to an initial goal for this thesis:

\section{To determine the effect of realistic current drift on routing protocols and $3 \mathrm{D}$ drifting UWSNs.}

Existing routing protocols are included in the study as they are directly affected by current advection. The role of a routing protocol is to establish paths, and the currents cause these paths to change. The routing protocols analysed in this thesis, Depth Based Routing (DBR) [205] and HydroCast [113], have also only been evaluated with simplistic land-based or depth-invariant current mobility models, and only their averaged performance has been reported. This leads to potentially misleading results, where it is unclear whether the level of performance is maintained throughout and persist afterwards. As nodes separate under drift, it is expected that end-to-end delay should increase, since packets must be relayed over longer distances to sinks, with consequent greater propagation times. In this thesis, their evolving performance over time is evaluated.

This required first achieving another goal:

2. To develop a general set of realistic 3D underwater node mobility models, representative of various ocean currents encountered at likely UWSN deployment sites.

As surveyed earlier, no realistic 3D current model with the resolution needed for analysing UWSN behaviour exists, requiring us to devise one for the study. This step was only taken reluctantly, as the author has a networking rather than an oceanographic background. It would have been preferable to use an accepted mobility model, but there was no such option. In making sure this was the only way to proceed, staff at New Zealand's National Institute of Water and Atmospheric Research (NIWA) were consulted [157]. The best current mobility model provided, coming from a numerical ocean model [167], had a horizontal resolution of $10 \mathrm{~km} .{ }^{1}$ This resolution was too low to model the neighbour interactions in an UWSN. Before

${ }^{1}$ NIWA are currently working on a model with a $1 \mathrm{~km}$ spatial scale 
proceeding to use the mobility model that we devised in network performance studies, we first validated it with an expert at NIWA [203].

In addressing Goal 1, results showed that the packet delivery rapidly degraded within a few hours due to nodes being carried by currents out of communications range with each other. The results are presented in Chapter 4. This suggests that the drifting UWSN concept needs to be discarded and that mobile nodes are required that can redeploy themselves to keep the network operational.

This led to the following goal:

\section{Investigate extending the lifetime of untethered 3D UWSNs using node mobility.}

This work looks at the number of nodes and energy consumed in mobility needed to keep a region of interest covered by a UWSN consisting of holonomic AUVs. This thesis also examines exploiting the mobility of UWSNs consisting of profiling floats for coverage. The unit cost of AUVs capable of fully omnidirectional motion is expensive, between US\$50,000-250, 000 [143]. In contrast, it is relatively simple to travel up and down in water by varying the buoyancy of a device. This type of mobility is employed by profiling floats, costing around US\$9000 [178] each. Although only vertically actuated, floats can utilize this to change their horizontal velocity due to the variation of currents with depth. By ascending or descending, the device can choose to drift at different speeds and directions given by the current velocity at that depth. Works looking at path planning for single floats indicate there is sufficient variability in the currents for floats to move to arbitrary locations [181].

\subsection{Thesis contributions}

In support of these goals, the following contributions were made:

1. This thesis derives a physically-inspired 3D mobility model for the Gulf Stream, based on the MCM model [32]. A scaled version of this model is also developed for coastal currents. 3D versions of the originally 2D MCM model have been proposed in the past but have been either depth-invariant $[57,113]$, when currents are known to change with depth [11], or no explanation for the extension has been given [14]. In our work, we go back to the oceanographic observations on which the MCM model is based to present a fully justified 3D extension for the current. 
2. Using this model, the connectivity and performance over time of a drifting 3D UWSN and pressure routing protocols are evaluated in simulation. This is the first time that their performance has been assessed in a realistic $3 \mathrm{D}$ current model.

3. A key contribution of this thesis is its investigation of profiling floats. Floats can only profile up and down, but by moving to different depths where the currents point in different directions they can travel to arbitrary positions [181]. Being an order of magnitude cheaper to fabricate, float networks have the potential to achieve similar coverage performance as much more expensive AUV networks, by also being able to move back to their initial deployment locations fully covering the RoI. Previous works have considered the problem of path planning for single $[93,180,182]$ and widely spaced 2D arrays of floats $[42,130]$, making this thesis the first to examine exploiting the mobility of UWSNs consisting of profiling floats for 3D coverage.

4. A float movement scheme is proposed with the goal of extending the time the UWSN is able to cover a 3D RoI. A major focus of our design is ensuring the scheme can be implemented in practical UWSNs. The scheme is evaluated in simulation, and compared with an AUV-based solution.

\subsection{Thesis structure}

The remainder of this thesis is organized as follows.

Chapter 2 provides additional motivations for UWSNs, in the context of pollution monitoring. This leads to an underwater oil plume tracking application being specified that acts as a unifying theme for this thesis. Evaluation scenarios employed throughout this work are based on this pollution monitoring scenario.

Chapter 3 presents our 3D current mobility models that we have developed for the

Gulf Stream (boundary currents) and coastal currents. These ocean environments are frequently the sites for pollution events, such as harmful algal blooms (HABs) that form off the coast around sewage outlets [75]. These models more realistically extend the 2D MCM model to 3D than current models in the literature, which are arbitrarily depth-invariant, by having real-life flow measurements be utilized for the vertical structure. This results in a current that is depth-decaying.

Our coastal current mobility model is utilized to advect a drifting 3D UWSN in Chapter 4. The simulated performance of pressure routing protocols and the 
connectivity state of the network is evaluated and discussed. The rapid decline in connectivity and routing protocol performance motivates the development of UWSNs consisting of self-propelled nodes that can oppose current drift. Within 3 hours, from a fully connected network, nodes have become so spread out that no paths to sinks exist.

Chapter 5 presents the practical node movement scheme we have proposed for extending the time a float-based UWSN is able to cover a 3D RoI, where profiler path planning has only been investigated for single floats or widely-spaced 2D arrays by existing works, and no works have modelled the energy consumed by floats. The pollution monitoring simulation carried out to validate the performance of our node movement scheme is described in Chapter 6. Our scheme is found to be able to sustain a level of area coverage throughout the entire length of the mission, whereas by the end of the mission other basic schemes have ceased any coverage of the RoI. Our solution is also shown to provide coverage more cost-effectively than using AUVs.

Finally, Chapter 7 summarizes the key findings of this thesis and suggests directions for future work. 


\section{Chapter 2}

\section{Background}

This chapter presents context information, to help the reader understand the spatiotemporal scale of UWSN applications. The material is illustrated utilizing the topic of pollution monitoring. The use of UWSNs for pollution monitoring is motivated in Section 2.1, which describes a growing pollution problem in the ocean. The ineffectiveness of existing sensing techniques for pollution monitoring is explored in Section 2.2, and then contrasted with the advantages of UWSN monitoring, Section 2.3. The chapter concludes in Section 2.4 with an important oil plume monitoring scenario, which will be referred back to as influencing the design of evaluation scenarios throughout the thesis.

\subsection{The need for pollution monitoring}

Our use of the ocean is a dichotomy. While we eat fish and swim in it, at the same time we dump our sewage in it and take a calculated risk in extracting oil and gas from it. Clean water is desirable in the former instances, yet we threaten to pollute the ocean with the latter uses. Wastewater introduced into the ocean can lead to harmful algal blooms (HABs), patches of anoxic or toxic water responsible for mass deaths in fisheries and even human fatalities from shellfish poisoning. Furthermore, the dangers of HABs and oil spills only appear to be increasing.

\subsubsection{Harmful algal blooms (HABs)}

Water contaminated with sewage and farm runoff contain high amounts of nitrates, phosphates and other nutrients that support increased growth of phytoplankton. When other conducive effects such as high temperature and sunshine also occur 
over such water, this can lead to critical conditions for plankton growth that allow concentrations of up to millions of algae per litre to accrue [75]. The density of plankton in the resulting algal bloom can become so great that they appear visible, forming cloudy patches on the surface of the water, an example of which is shown in Figure 2.1, spread over several hundreds of metres or more. The conditions enabling such growth may coincide only for a short while, and the bloom may disappear as rapidly as it appeared, after a few hours, days or weeks [86].

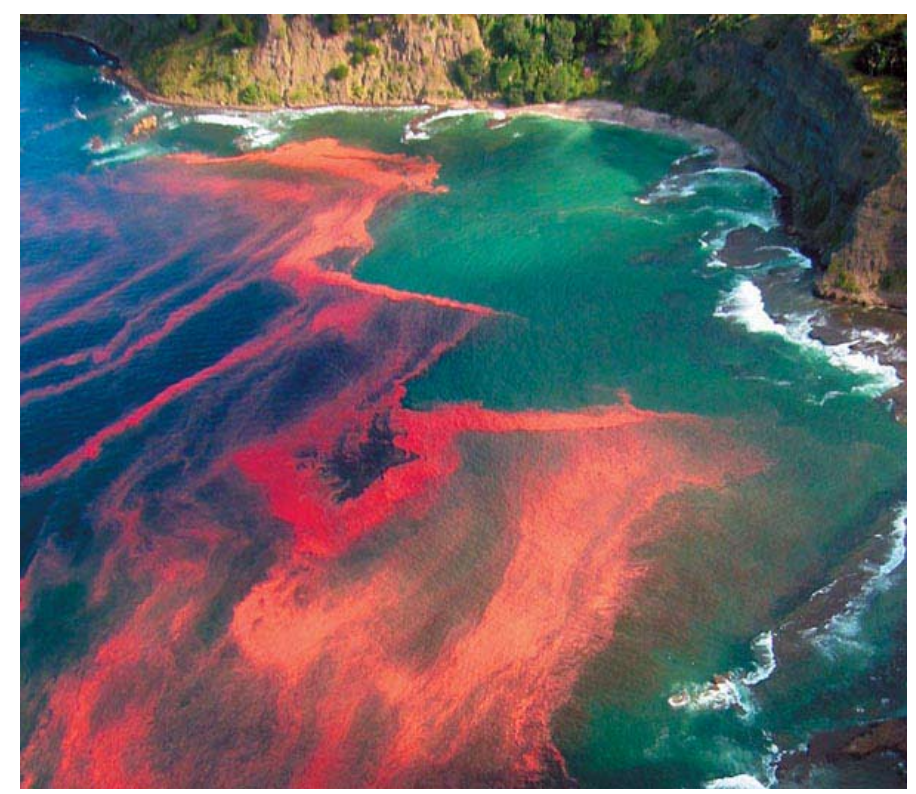

Figure 2.1: Biblical "rivers of blood": algal bloom off Leigh, near Cape Rodney in New Zealand. Image from [69].

Algal blooms are a concern because some can become harmful. As plankton die off, bacteria decomposing their bodies consume oxygen in respiration. The concentration of algae can reach such levels that the area becomes oxygen deprived, causing fish within it to suffocate [75]. This becomes especially problematic for fish that cannot move out of the way of the bloom, namely those fenced in marine farms. Aquaculture has seen rapid growth in the past 40 years, from nearly $0 \%$ of world fish production in 1970 to $40 \%$ in 2000 [76], so that for some species, like salmon, a specimen bought at a shop is more likely to have been farmed rather than caught. In part this is caused by rampant overfishing of wild stocks, which has seen the median populations of the world's fisheries declining by $83 \%$ in the last 25 years [82], and causing farming to be more profitable as fishing ships have to travel further and spend longer out at sea to return with a full load. A HAB passing through a cultivated area can be devastating. Among cases surveyed by Islam and Tanaka [87], 
a 1998 bloom along the coasts of Hong Kong and south China killed over 80\% of farmed fish, and a bloom in France wiped out an entire farm.

Some algae can also produce neurotoxins, creating a danger to humans. Because shellfish absorb and accumulate the toxins in their bodies, eating shellfish affected by an algal bloom can prove fatal. While humans can be warned to avoid shellfish during these times, sea lions and birds cannot be so fortunate [171], and have been known to die from algal bloom poisoning [75]. The frequency with which blooms occur has increased and is expected to further increase, as a growing human population produces more runoff and climate change is predicted to raise water temperatures that are more conducive to algal blooms forming [75]. For instance it is feared that the warming of the oceans will allow toxic Pyrodinium bahamense blooms to form off Australia. Currently the species is limited to waters around and north of Papua New Guinea, with the waters further southward being too cold for it to thrive. However, fossil records show that during warmer periods in the past, the algae had extended as far south as Sydney Harbour. The increase in HABs with human population growth is consistent with existing findings, such as in Hong Kong Harbour between 1976-1986. During this period, which coincided with the city's population expanding 6 -fold, the number of blooms per year increased 8-fold.

\subsubsection{Oil spills}

Another area where the amount of pollution being generated is likely to increase is in oil spills, due to the rise in offshore oil and gas exploitation. From comprising $0.10 \%$ of US oil production in 1954, the importance of offshore oil production in the petroleum industry has grown dramatically, with, as of 2011, $25 \%$ of US oil production coming from oil platforms [9]. The results of an oil spill can be disastrous as demonstrated by the 2010 British Petroleum (BP) or Deepwater Horizon incident in the Gulf of Mexico, pictured in Figure 2.2. The accident resulted in an estimated 360 to 700 million litres of oil to leak into the ocean, making it the worst marine oil spill in history [176]. Oil and the chemical dispersants used to treat it are known mutagens. Following the disaster high incidences of deformed sea life being caught were reported, from clawless crabs, shrimps with tumours on their heads to eyeless fish, with a study carried out by the University of South Florida finding in some areas that $50 \%$ of fish had lesions from a pre-spill figure of $0.01 \%$ [89,202]. In 2015 $\mathrm{BP}$ was ordered to pay 18.7 billion dollars for damages caused by the spill to the environment, the largest environmental fine in US history [164]. 


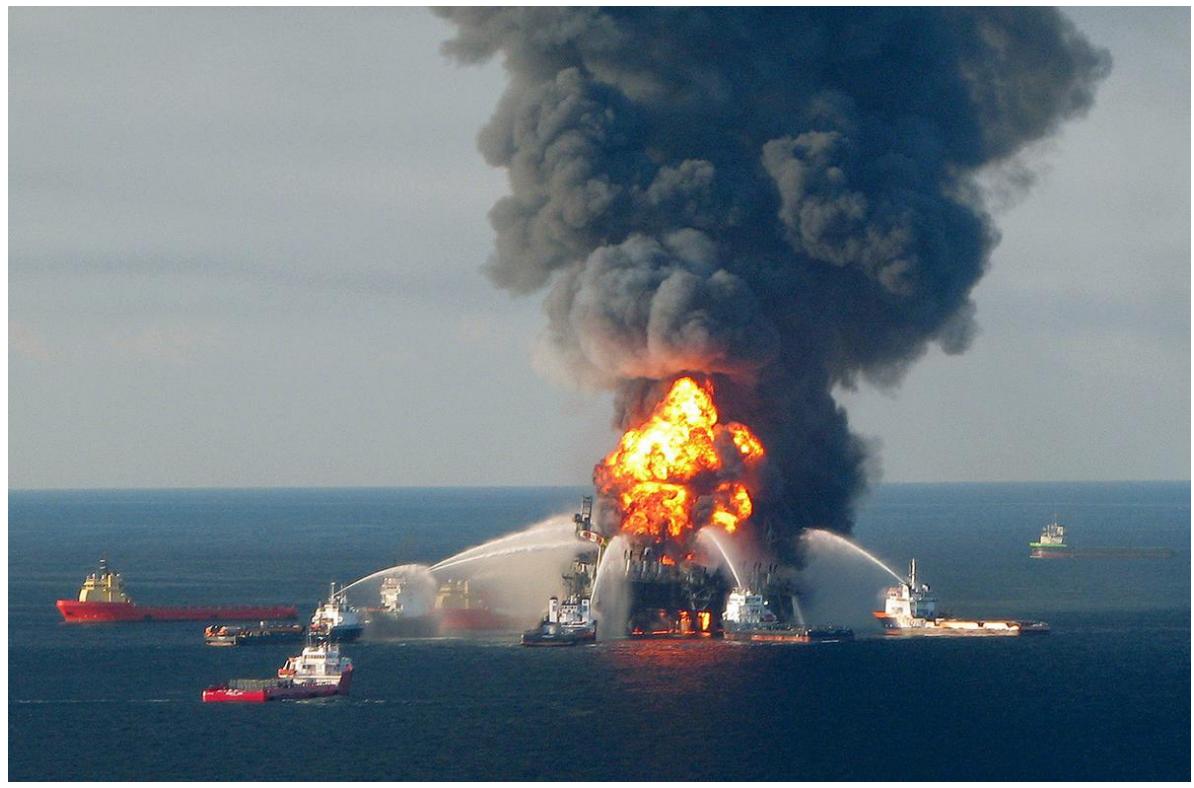

(a)

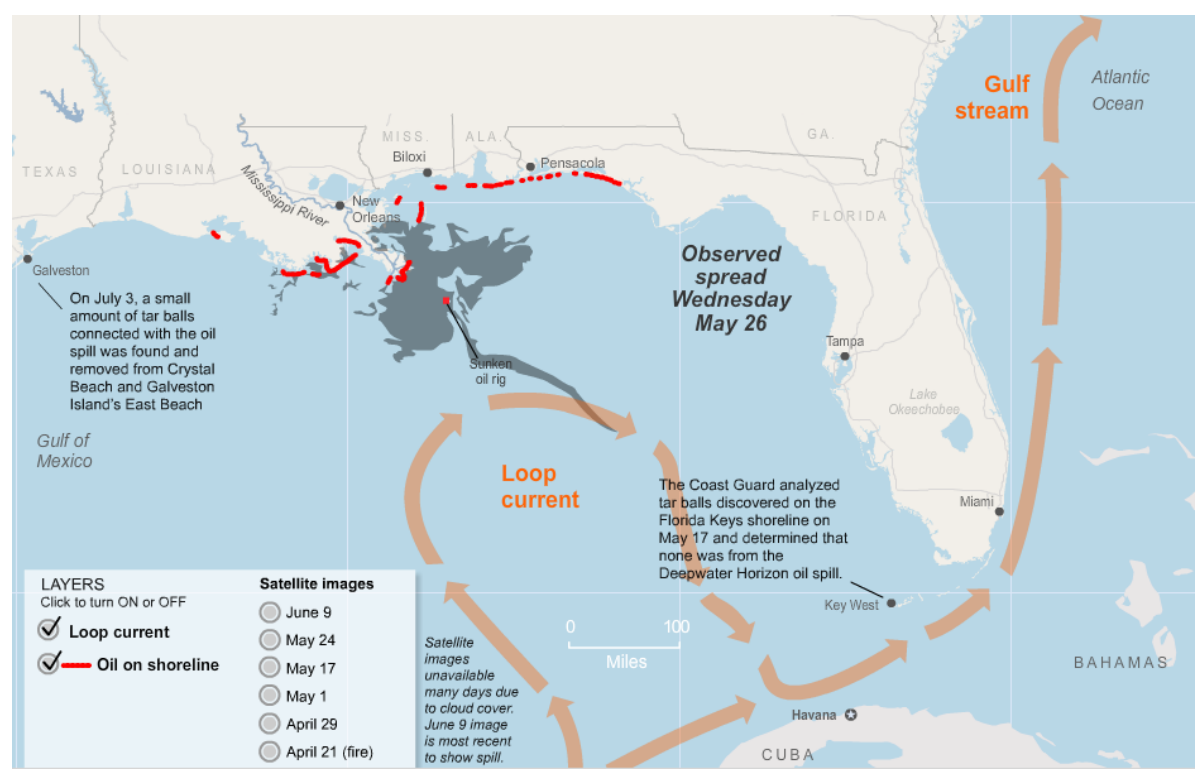

(b)

Figure 2.2: The 2010 Deepwater Horizon oil spill. (a) The burning oil platform on April 21, 2010, one day after the explosion causing the spill. Image from [191]. (b) Observed spread of oil on the surface on May 26, 2010, from [198].

\subsection{The present state of underwater sensing}

Although marine pollution incidents are only set to increase in number, existing capabilities for pollution monitoring are limited. Images from satellites or planes can 
be used to track the extent of a HAB or oil spill over a wide area, which show up as discolourations in the water. However, these observations are limited to the surface and cannot provide any information about the chemical composition of the water. For in-situ sensing, currently this is carried out using ships [107], mobile unmanned sensor platforms such as autonomous underwater vehicles (AUVs) [29] and floats [160] that are un-networked when they are underwater, and moored sensors $[41,153]$, as shown in Figure 2.3.

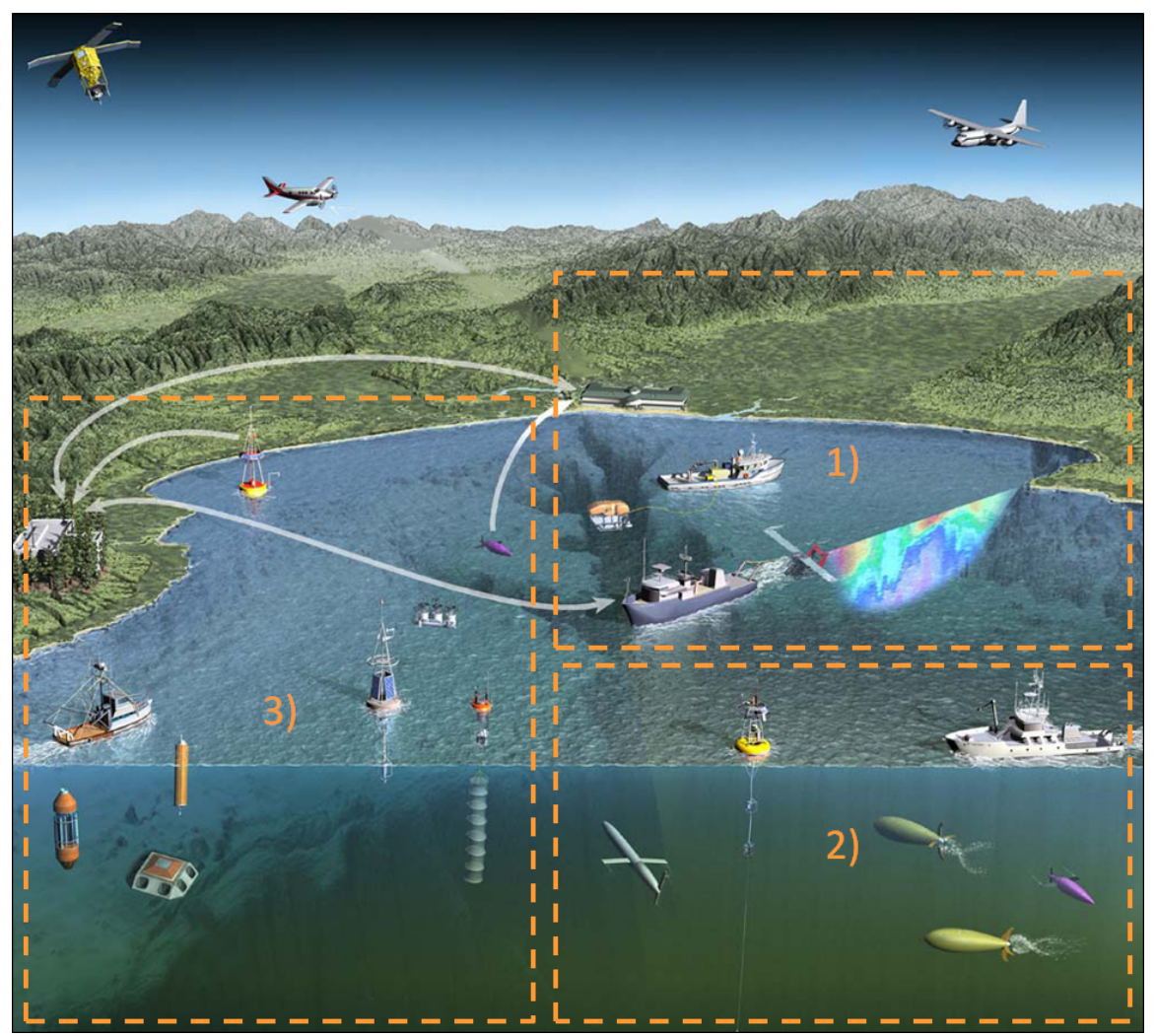

Figure 2.3: Present day methods for in-situ underwater sensing, from [21], showing clockwise from right: sensing using 1) ship-based surveys, 2) ship-deployed AUVs and 3) moored sensors and buoys, and drifters and floats. From the sky, features may be tracked visually by satellite or plane.

These approaches are severely constrained in the scale of the area they can sense and their availability.

Both ships and AUVs are expensive, and as a result are typically deployed singly [29]. A large ocean-going research vessel costs around US\$10 million to construct, as well as requiring US $\$ 25,000 /$ day in crew and fuel expenses to operate [143]. The unit cost of an AUV ranges from US\$50,000 - 250, 000 [143]. This means only a small part of a pollution feature, which may be enclosed within a $1 \mathrm{~km} \times 1 \mathrm{~km} \times 1 \mathrm{~km}$ box in the case of a HAB [43], can be sensed at any one time. A ship is able 
to assess the water quality underneath it using rosette sampling [29]. In rosette sampling, a device consisting of a circular row of buckets is lowered beneath the vessel. The buckets are opened at different depths to collect water samples, that are then analyzed on board.

Multi-vehicle arrays have been considered, where owing to the high cost of unmanned underwater vehicles (UUVs) and the ships needed to deploy them, the devices are either widely spaced apart or the number employed is small. In Fiorelli [62], an experiment with three gliders is carried out where the vehicles are tasked with sampling a distributed area while being separated $3 \mathrm{~km}$ apart. A global array of floats is maintained in the Argo program [160], where each node is on average $300 \mathrm{~km}$ apart. This highlights a second problem with UUV sensing. While underwater, devices can only communicate acoustically. This communication is limited in range to around $350 \mathrm{~m}$ [118]. An UUV can only co-ordinate with other vehicles and report back its sensing results to land after having surfaced in order to communicate via satellite. The time between surfacings may be several days [160].

For long-term studies, the conventional approach has been to deploy moored sensors [153]. Mooring lines are limited in length, restricting their use to shallow waters, and are complex to install, requiring long setup times [121]. This makes them unsuitable for sensing suddenly occurring events like an oil spill or a bloom. By the time the moored sensors have been set up the bloom, lasting a few days, may have already ended. Furthermore, since pollution is suspended in water and drifts with the underwater currents, a static field can only briefly monitor the feature before it leaves the area.

\subsection{Drifting underwater wireless sensor networks}

Drifting underwater wireless sensor networks (UWSNs) appear to overcome many of the problems related to existing sensing methods, making them well-suited to pollution monitoring. Nodes can be rapidly air-dropped onto an emerging event and then follow it as it drifts. Furthermore, as nodes are connected to end-users via sinks, sensed data can be received and acted upon immediately, rather than having to wait days for an AUV to surface [160] or months, when moored sensors are retrieved at the end of their mission [153].

With UWSNs consisting of hundreds to thousands of nodes, sensors can be placed throughout the pollution feature. Monitoring the entirety of a HAB has the advantage, as identified by Das et al. [43], of being able to measure the nutrient flux 
into the bloom and its spatiotemporal dynamics, allowing the conditions that lead to a bloom forming and to continue growing to be better understood. To achieve this, Das et al. [43] look at mapping a bloom with a single AUV that repeatedly traverses each part of the feature in various survey patterns, such as a lawnmower pattern. The AUV's speed, $1.2 \mathrm{~ms}^{-1}$, compared to the $1 \mathrm{~km} \times 1 \mathrm{~km} \times 1 \mathrm{~km}$ size of the HAB, means that each point can only be revisited with very coarse temporal resolution (once in several hours). In contrast, a UWSN promises being able to place nodes at each part of the RoI, so that all points can be continuously monitored. The poor resolution of single AUV sampling, along with other sensing methods being limited in the area they can sense has meant that present-day understanding of overall algal bloom dynamics is poor to non-existent [180].

\subsection{Motivating scenario: underwater oil plumes}

This section presents an example of where UWSN sensing could have made a significant difference. Due to its relevance, being related to the recent Deepwater Horizon oil spill, and novelty, many of the evaluation scenarios that are studied throughout this thesis address this application. In the literature, simulations evaluating drift $[32,119]$ and routing [113,205] and localization [59] protocols have been carried out for various scenarios. These scenarios are unreferenced and hence have seemingly arbitrary deployment area sizes and numbers of nodes. This thesis relates the work to a concrete scenario, making the reasons for certain currents, region of interest sizes and traffic models clear. We are the first to consider this important application.

The Deepwater Horizon disaster was not only the largest [176] and costliest [164] marine oil spill in history. It was also one of many firsts. It was the first oil spill to occur at significant depth [193]. An explosion at the rig caused oil and gas to stream, uncontrolled, directly out of the wellhead at $1500 \mathrm{~m}$ depth and into the water. In an attempt to treat the spill, dispersant was injected directly into the stream at the wellhead. This was the first large-scale application of dispersants in deep water, with no data being available on the outcome of this action [107]. The treatment led to some of the oil, rather than rising to surface, being sequestered at depth, forming drifting underwater oil plumes of suspended oil droplets as shown in Figure 2.4. These clouds were a new, previously unobserved phenomenon [29]. Plumes reaching over $35 \mathrm{~km}$ in length and $2 \mathrm{~km}$ in width between $1000-1200 \mathrm{~m}$ and $50-500 \mathrm{~m}$ depths were observed [29] and were still present months later [107]. At the time it was feared that bacteria consuming the oil, using oxygen to do so, would 
lead to anoxic conditions, giving rise to large "dead zones" inside the plumes where marine life would suffocate [68].

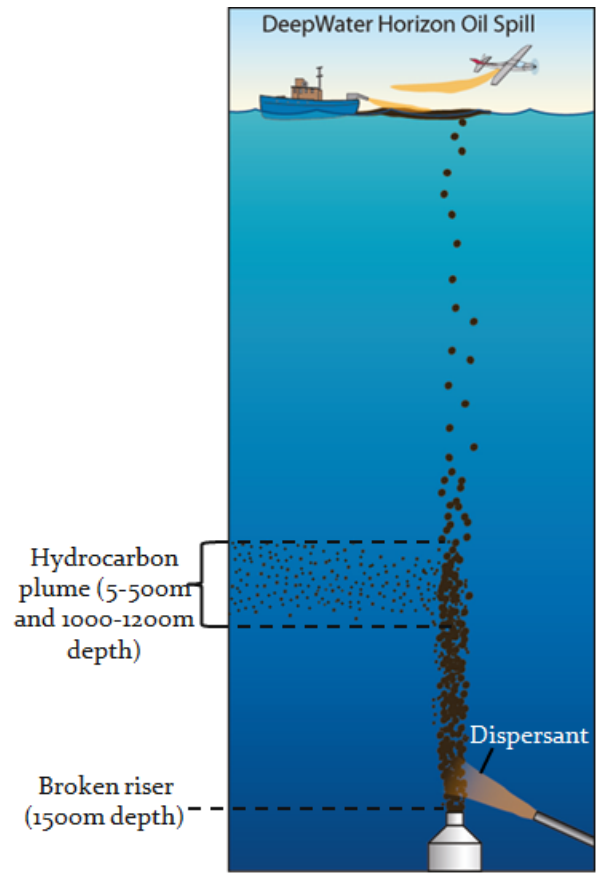

(a)

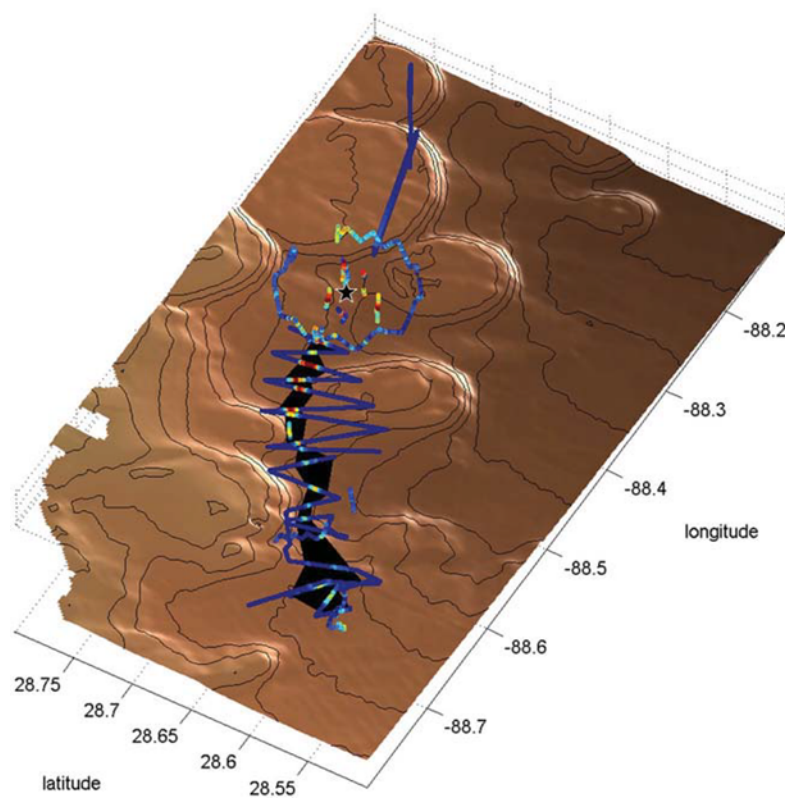

(b)

Figure 2.4: Underwater oil plumes arising from the Deepwater Horizon disaster. a) Schematic, from [107] b) Survey path (blue) taken by the Sentry AUV in [29] of the oil plume (black), showing horizontal scale, and that the AUV was only able to take transects of it.

Obscured from view unlike the surface oil spill, the plumes were not detected until a ship survey on May 15, one month after the explosion [68]. This delay can be attributed to the logistics of provisioning a ship and gathering a crew. In remote areas away from a port, travel time must also be taken into account. The plumes were surveyed by ship and single AUVs [29] that could only sample small sections of it, and the fate of oil droplet clouds remains poorly understood [193].

In this thesis, the use of UWSNs to monitor underwater oil plumes is suggested. A drifting or mobile UWSN can be deployed to continuously sense a large patch of the plume and follow it to observe its dynamics. Nodes can be airdropped into the spill immediately after the incident and self-organize into a network after landing. Potentially, if the technology had been available, a UWSN could have identified plumes days rather than a month into the incident. With this information, crews could have determined if this behaviour was intended and altered their approach to treating the spill with dispersants. 


\section{Chapter 3}

\section{A realistic 3D depth-decaying meandering current mobility model}

Thus far in this thesis, we have presented background material on UWSNs and the need for realistic 3D current mobility models to be able to accurately assess the performance of different UWSN design strategies. A network architecture definition of a UWSN was given in Section 1.1, and the new capabilities that this paradigm offers over exiting underwater sensing techniques were discussed in Chapter 2. UWSNs offer the ability to sense a wide area with high granularity, ease of deployment and reusability [113], enabling applications in pollution monitoring, distributed tactical surveillance and oil/gas field exploration [11,41]. The potential offered by these capabilities has attracted increasing research interest, with the development of network schemes (deployment strategies [32], and medium access control (MAC) [97], localization $[58,190]$, routing $[18,113,134]$ and synchronization protocols [189]) that are optimal for this type of network.

There are still many technical challenges to be overcome though [143], and UWSNs have yet to be deployed [58], resulting in these proposed protocols being evaluated in simulation. In simulations, it is important that those factors significantly influencing performance are accurately modelled, so that a useful evaluation is obtained of how the protocol is likely to perform in actual deployment [30]. Mobility is one aspect of UWSNs, where nodes are untethered and each node drifts with the currents at the speed of the fluid parcel it is occupying [44], that greatly impacts performance. Coverage evaluates the percentage of the RoI that is being sensed by at least one node and is treated in the literature as one of the most important measures for the 
quality of service (QoS) provided by a sensor network [52], whose purpose is to carry out a sensing task. Coverage depends on the node deployment, which is affected by mobility. For example, current drift can cause all nodes to move out of sensing range of a point leaving a coverage hole [207], or an uncovered part of the network.

To correctly account for the impact that mobility has on coverage, a representative current mobility model needs to be specified in the simulation. A current mobility model defines the velocity of each node due to the current. Existing 3D current models come with significant limitations, however. As surveyed in Section 1.2, 3D current models in the literature are restricted to the output of numerical ocean models $[42,130,179,182]$, which are too low resolution to reflect the interaction between neighbouring nodes, and land-based synthetic mobility models [35, 36, 119, 204, 205] such as random walk or random waypoint where nodes move independently of each other. In reality, nodes are advected underwater by a common flow, which can be represented by a current velocity field [37]. The movement of sensors is thus highly correlated and this is not reflected in land-based stochastic mobility models, although these models continue to be used. An argument for why given by authors [119] is that currents vary so widely between regions (for instance the flow in a river compared to the semi-periodic in-out tidal flow along a beach) that no current mobility model could represent all environments. Thus, while the flaws of a stochastic model are acknowledged, Liu et al. uses it to obtain generic mobility results for their redeployment protocol. Chirdchoo et al. [36] represents the currents with random walk in their work. The explanation given, with no evidence provided, is that underwater sensor nodes experience random movement due to the unpredictable ocean current.

The lack of accurate 3D current mobility models across various ocean environments has been noted in several surveys $[58,190]$. One possibility is to develop 3D current mobility models from 2D models in the literature. A number of $2 \mathrm{D}$ current mobility models have been applied to research into sensor networks for sea surface monitoring that, unlike stochastic and numerical ocean models, are more realistic and not restricted to low resolutions. These models are more likely to reflect typical environmental conditions by being derived from records of lake [121], tidal [71] and coastal flows [32], and velocities are defined at all points to arbitrary resolution by a function [32,71]. One such model, Meandering Current Mobility model (MCM) [32] has gained widespread acceptance among the UWSN scientific community and been used in performance studies of various routing protocols $[113,134]$, and node movement $[121,172]$ and localization schemes [59]. MCM is based on observations of the 
Gulf Stream current [140, p. 107] and is proposed to represent coastal currents. At the time of its publication, the authors claimed that "to the best of our knowledge this is the first physically-inspired mobility model used in the analysis of mobile underwater sensor networks [32]" and as recently as 2014 the MCM model has been described as "the only UWSN mobility model available in the literature" [123].

In this chapter, we develop our own extended 3D version of MCM that is able to describe the current velocity field across a continuum of depths rather than just the sea surface layer as in the original model. The MCM model is chosen because of the availability of hydrographic data for the Gulf Stream, its popularity, and relevance to likely areas where UWSNs are likely to be deployed. The Gulf Stream was pictured in Figure [?] for the pollution monitoring scenario motivating this thesis, and a major concern at the of the time of the Deepwater Horizon disaster was that oil would reach the Gulf Stream and be carried throughout the East Coast of the United States [198]. A number of other authors have come up with 3D versions of MCM [14,59, 113, 134], in all cases depth-invariant. These models are of questionable validity, however. No justification, whether empirical, theory-based or otherwise, was given for the 3D extensions by their respective authors, and the depth-invariant natures of the models are inconsistent with the structure of the Gulf Stream and ocean currents in general.

A key difference separating our work from these existing 3D models is that the $3 \mathrm{D}$ structure of our current is defined to reproduce the measured velocity profile of the Gulf Stream in Halkin and Rossby [72] , and so the validity of our work is based on actual readings. In the original MCM model, the current is represented by a time-varying, sinusoidally meandering jet pattern in which the velocity of the flow falls off further from the centre of the jet. The original MCM current is expressed in terms of variables controlling how time-varying the current is and its envelope (or shape). These can be adjusted to more accurately resemble a specific current by being set to match observed values, and by doing this MCM has been employed to represent currents across many different spatial scales [25,32]. However, the structure of the velocity falloff within the jet or velocity profile is fixed. In this work, we rewrite the current expression as a function of the jet velocity profile. The advantage using our expression over MCM grants UWSN researchers is the ability to tweak the velocity profile to match the underwater environment of their scenario, where we determine through rewriting the equation that the Bickley jet [23] is utilized as the velocity profile in the original MCM model. The Bickley jet is the velocity falloff in an idealized situation, the motion that arises from a jet issuing from a long narrow orifice into a fluid at rest. A 3D MCM current model with a more realistic 
velocity falloff is proposed by us, where an actual measured profile of the Gulf Stream current is employed to obtain the model's profile. Two models are developed, one for the Gulf Stream and a rescaled version of this to fit typical coastal currents. The resulting currents are depth decaying, decreasing in velocity with increasing depth, and this results in some unique effects when these models are employed in Chapter 4 to evaluate the performance of routing protocols. The slower velocity of nodes at greater depths causes them to lag behind the surface sinks in the network and rapidly lose connectivity.

The rest of this chapter is organized as follows. Sections 3.1-3.4 review background and related work. After going through some preliminaries for the notation used in this chapter in Section 3.1, the original 2D MCM model is introduced in Section 3.2. The ocean regions that MCM is intended to represent are described. 3D extensions of MCM in the literature are surveyed in Section 3.3, where it is found that existing models have portrayed the strength of the current as remaining constant with depth. Section 3.4 argues that this is likely rarely the case, based on current measurements and present-day theoretical understanding of the oceanic processes giving rise to currents, which indicate that in general currents decrease in intensity with increasing depth. Our novel 3D extension of MCM is developed from Sections 3.5 onwards. In Section 3.5, the original 2D MCM model is analysed to obtain an expression for the jet velocity profile, and the model is rewritten as a function of the profile. This form allows currents with custom velocity profiles to be specified. Section 3.6 presents our more accurate extended 3D MCM model for the Gulf Stream that has a velocity profile based on the measured structure of the Gulf Stream. Another model for coastal currents is developed by us in Section 3.7 by scaling our Gulf Stream model. The limitations of our current models are also examined in these sections, where the flaws of using scaling to represent currents arising from completely different oceanic processes than the original flow are discussed. Finally, Section 3.8 concludes the chapter.

The contributions of this chapter are summarized: 1) We derive an intuitive expression for the velocity profile of the MCM jet. This enables environment-specific profiles to be used, whereas the existing model could only reproduce Bickley jets. 2) We are the first to analyse the realism of both the original MCM model and extensions of it in the literature. Past models' assumptions of a depth-invariant flow are found to be incorrect based on oceanographic data. 3) We develop a depth-decaying current model from MCM that is likely a more accurate representation of the Gulf Stream current that MCM was designed for, due to the integration of real-life measurements 
of the current in the model, and is 3D. A scaled version of this model for coastal currents is also proposed.

\subsection{Preliminaries}

Standard convention in geophysical fluid mechanics is to define the current velocity for a local Cartesian coordinate system with respect to the rotating Earth, where $x$ is to the east, $y$ is to the north, and $z$ is up. The components of velocity in the $x, y$ and $z$-directions are denoted $u, v$ and $w$ respectively [185]. The eastwards velocity is also known as the zonal velocity, while the northwards velocity is often referred to as the meridional velocity. We similarly adopt this terminology throughout the thesis. The terms region, static or world [43] frame are also used to denote a frame that is fixed relative to the rotating Earth.

\subsection{The Meandering Current Mobility model (MCM)}

In this section, the MCM model is described. Over the course of this chapter, a more realistic, 3D version of the model is proposed. The MCM model [32] is a current mobility model for UWSN simulations designed to be representative of coastal currents. MCM is an example of a kinematic model: a model where the current velocity field is defined by a function [165]. In the MCM model, the current velocity field is specified by a streamfunction proposed by Bower [25], and later extended by Samelson to add fluctuating meander amplitudes [165], for the Gulf Stream current $[25,32]$

$$
\psi(x, y, t)=-\psi_{0} \tanh \left[\frac{y-y_{c}}{\lambda / \cos (\alpha)}\right]
$$

where the current velocities (in $\mathrm{ms}^{-1}$ ) in the $x, y$ and $z$-directions are given by

$$
\begin{gathered}
u(x, y, t)=-\frac{\partial \psi}{\partial y} \\
v(x, y, t)=\frac{\partial \psi}{\partial x} \\
w(x, y, t)=0
\end{gathered}
$$


for a vertical current velocity of zero. For reasons of consistency, we use Bower's dimensional notation for the streamfunction [25]. In Section E.1, we show that this is equivalent to the nondimensionalized streamfunction given in the paper proposing MCM [32] so that the two representations can be used interchangeably. The Gulf Stream is pictured in Figure 3.1 and is a western boundary current, part of the North Atlantic Gyre, that flows along the East Coast of the United States from the Straits of Florida to the Grand Banks off Newfoundland [140, p. 107], or a distance of approximately $3000 \mathrm{~km}$.

The velocity field describes a time-varying, sinusoidally meandering jet propagating eastward at constant speed, as shown in Figure 3.2. Visually, the current model appears similar to the Gulf Stream in Figure 3.1. The current forms a jet, a directed flow in which the current velocities at adjacent points are approximately parallel. The velocity is not constant along the cross-section of the jet but increases closer to the centre of the jet. The fixed width jet is deflected sinusoidally, where the meanders have fixed wavelength but variable amplitude that changes periodically between fixed minimum and maximum values.

The parameters in the streamfunction are related to the sinusoidally meandering current pattern by

$y_{c}=B(t) \sin \left[k\left(x-c_{x} t\right)\right]$, the $y$-coordinate of the centre of the jet at $(x, t)$.

$\alpha=\tan ^{-1}\left\{B(t) k \cos \left[k\left(x-c_{x} t\right)\right]\right\}$, the angle that the centre of the jet makes with the positive $x$-direction.

$\lambda$, the half-width of the jet in metres.

$\psi_{0}$, the scale factor: $s_{c}=\psi_{0} / \lambda$ gives the magnitude of the downstream speed in the centre of the jet, which is also the maximum downstream speed.

$c_{x}$, phase speed or speed of propagation of the jet pattern.

$L$, the wavelength of the jet pattern, where $k=2 \pi / L$ is the wave number.

$B(t)=\left\{\begin{array}{ll}B & \text { (in Bower's model) } \\ A+\epsilon \cos (\omega t) & \text { (in Samelson's model) }\end{array}\right.$, the amplitude of the jet in metres.

$T=2 \pi / \omega$, the modulation period of the time-varying jet amplitude.

These parameters control the shape, size and how time-varying the current is, and different variable sets for MCM matching various ocean environments have been 


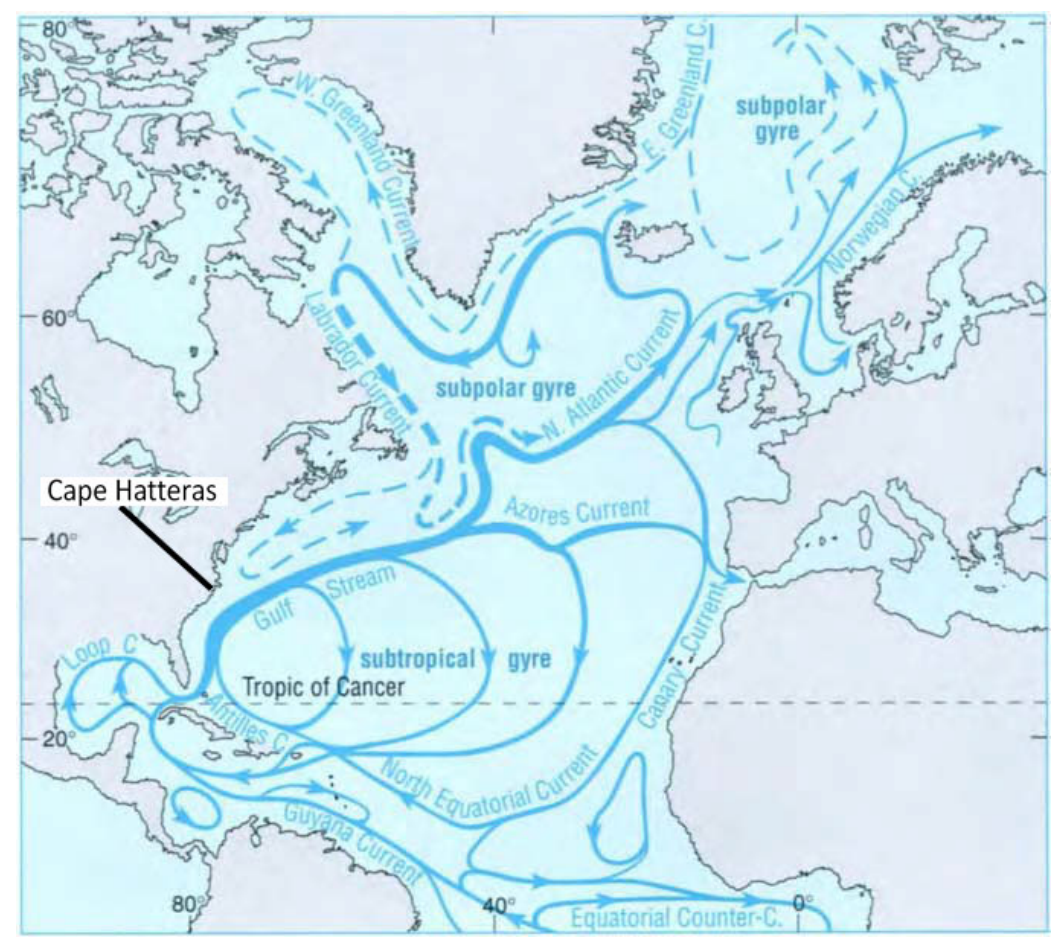

(a)

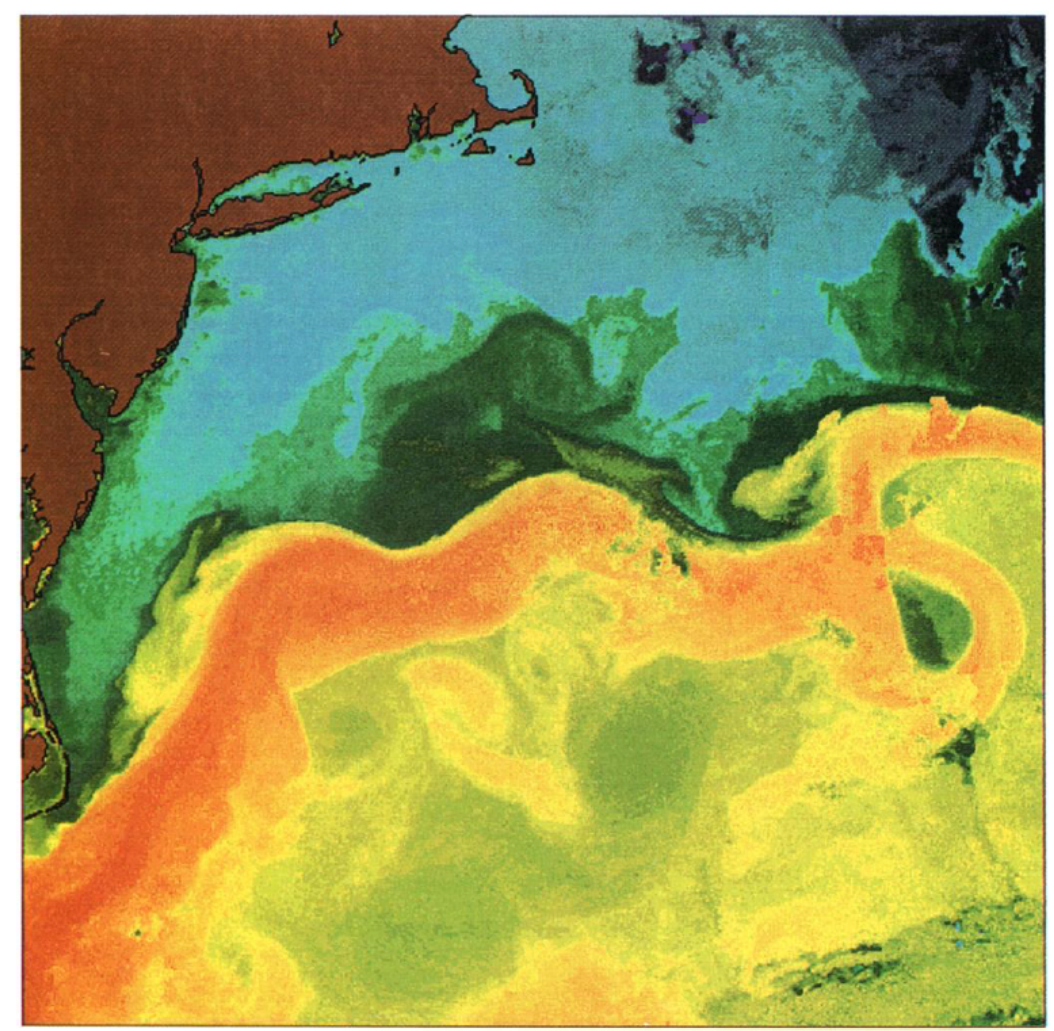

(b)

Figure 3.1: a) Gulf Stream in relation to other upper ocean circulation systems in the North Atlantic Ocean, from [140]. b) Sea Surface Temperature (SST) satellite image of the Gulf Stream current off Cape Hatteras, clearly showing as a meandering jet, from [55]. The position of Cape Hatteras, also the approximate deployment area for the floats used in Bower et al. [25] to obtain the observations on which their model is based, is annotated in a). 


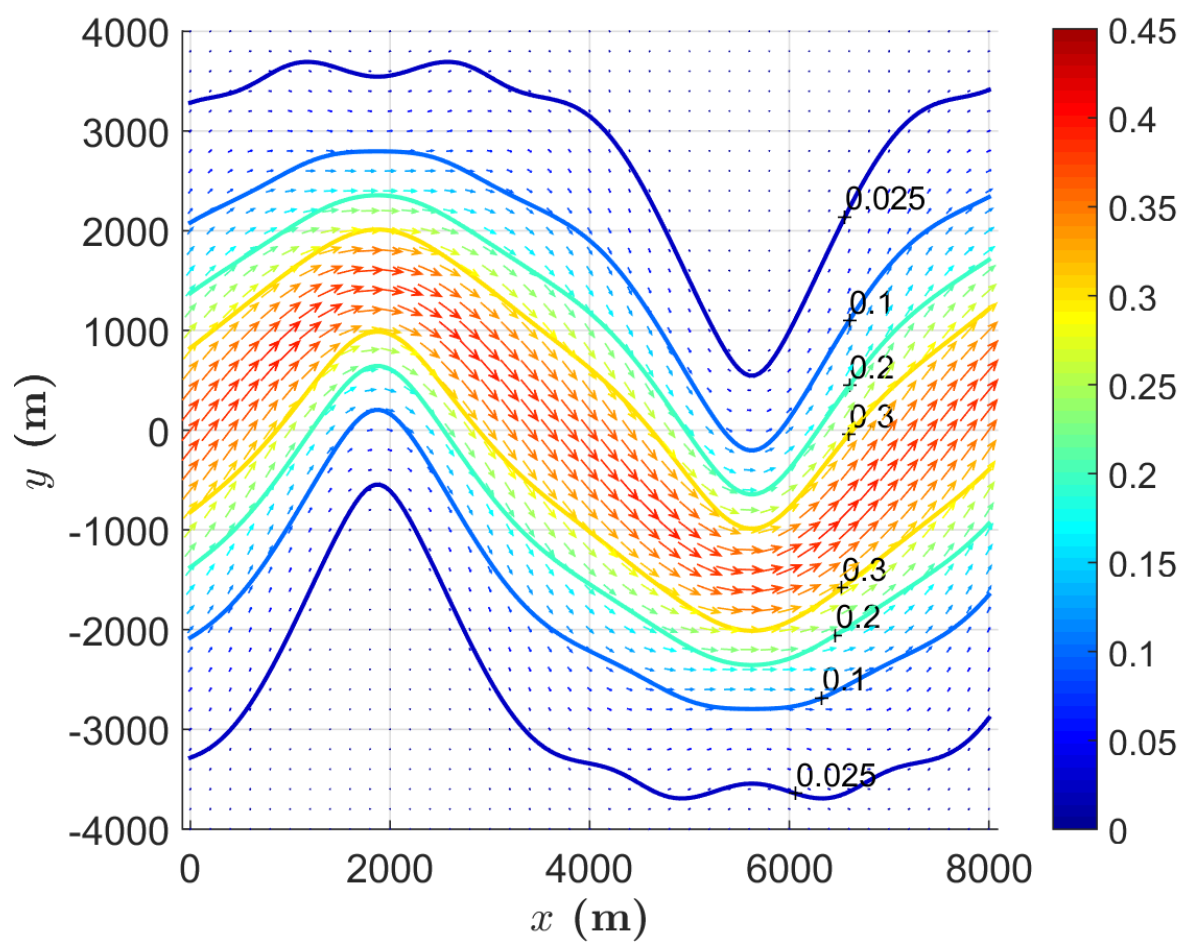

(a)

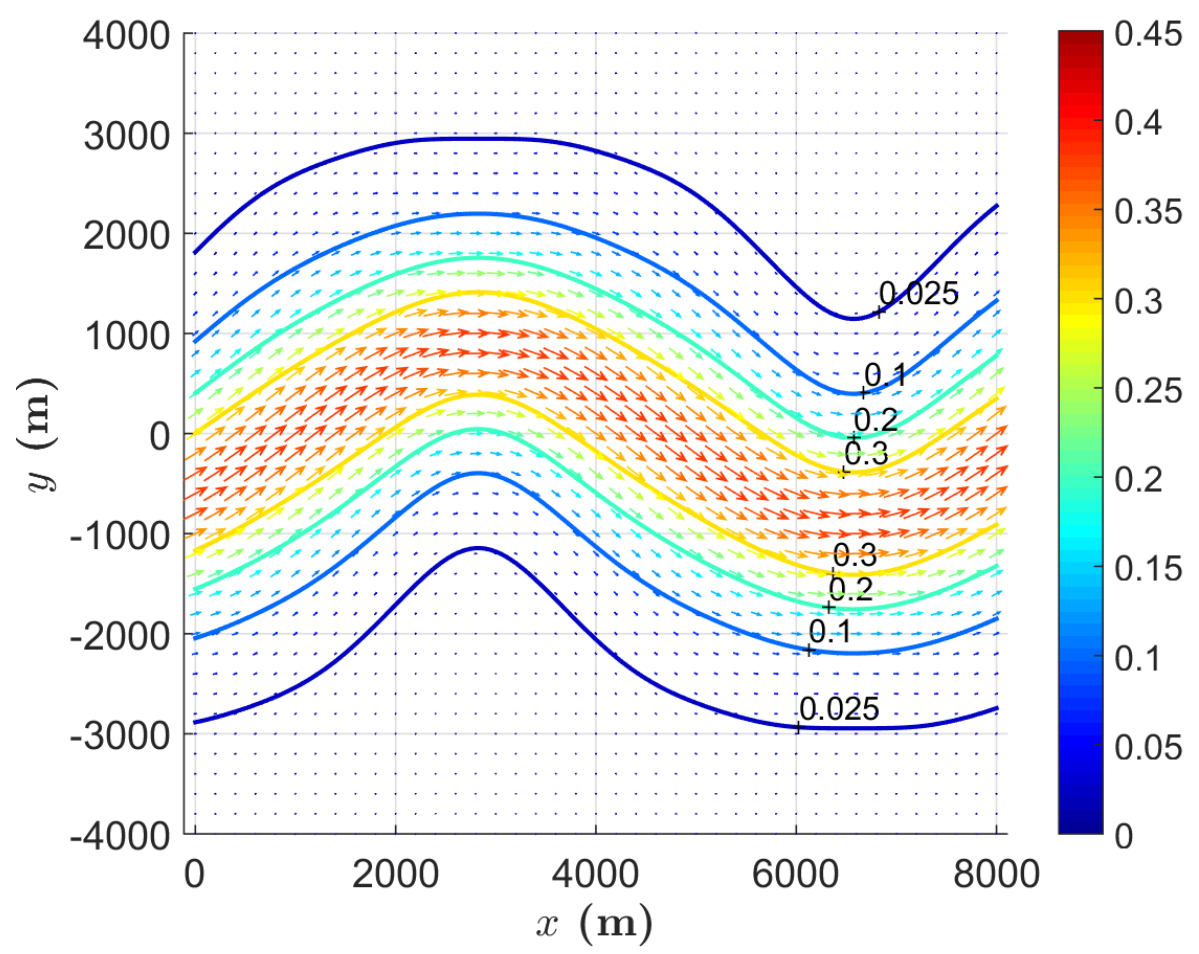

(b)

Figure 3.2: MCM current velocity field at a) $t=0$ and b) $t=5.7 \mathrm{~h}$, with (3.1) streamfunction parameters set as given by Table 3.2. Contour lines of fixed current speed and the magnitudes of individual velocity vectors, coloured based on their value on the scale to the right, show how speed decreases the further out from the jet. In the half-period spanned, the current pattern is shown, amplitude-wise, at its a) least and b) greatest extent. The jet pattern can be seen to be shifted right over time. This is due to the jet being eastward-propagating. 
proposed. The settings used by Bower [25] for the surface Gulf Stream current are listed in Table 3.1. Settings asserted to be representative of a typical coastal current are given by Caruso et al. [32], and which are repeated here in Table 3.2. The region of water between the coastline and up to $10 \mathrm{~km}$ out to sea, which is often watched by HF radar stations, is specifically identified by Caruso et al. [142] as the environment on which these settings are based. An example of the surface current velocity field in such an environment is shown in Figure 3.3 and is superficially consistent with the MCM model, displaying similar meandering directed flows that are in this case along-shore. The central speed, $2.31 \mathrm{~ms}^{-1}$, and width, $80 \mathrm{~km}$, of the Gulf Stream MCM model are consistent with (the same order as) the actual current. The Gulf Stream is typically some $100 \mathrm{~km}$ wide with surface velocities in excess of $2 \mathrm{~ms}^{-1}[140$, p. 85]. In comparison, the coastal current MCM model has a centre speed of $0.39 \mathrm{~ms}^{-1}$. This model portrays a much slower and thinner jet .

Table 3.1: Surface Gulf Stream MCM parameters laid out by Bower [25]

\begin{tabular}{lr}
\hline Parameter & Value \\
\hline Central downstream speed $\left(s_{c}\right)$ & $2.31 \mathrm{~ms}^{-1}$ \\
Wavelength $(L)$ & $400 \mathrm{~km}$ \\
Jet half-width $(\lambda)$ & $40 \mathrm{~km}$ \\
Phase speed $\left(c_{x}\right)$ & $0.12 \mathrm{~ms}^{-1}$ \\
Jet amplitude function $(B(t))$ & $B$ \\
$B$ & $50 \mathrm{~km}$ \\
\hline
\end{tabular}

The streamfunction was developed by Bower [25] after studying the trajectories of 37 current-following RAFOS floats that she had deployed in the Gulf Stream off Cape Hatteras and was able to successfully explain the broad-scale features of the paths traced by the floats. The key flow behaviours observed were: fluid parcels more readily left and were absorbed into the jet 1) at lower depths and 2) when the jet pattern was more curved, and 3) fluid parcels did not maintain a fixed position relative to the centre of the jet as they were carried downstream, but move away from the leading edge of a meander and toward a meander's trailing edge, as depicted in Figure 3.4. The leading edge here is used to denote the envelope of the meander that is further ahead along the overall downstream flow of the current looking at sections aligned perpendicular, also known as cross-stream, to the jet Sometimes fluid parcels moving toward the leading edge cross out of the jet altogether, resulting in: 4) the principal locations for entrainment into the jet and detrainment out of the 
Table 3.2: Coastal current MCM parameters laid out by Caruso et al. [32]

\begin{tabular}{lr}
\hline Parameter & Value \\
\hline Central downstream speed $\left(s_{c}\right)$ & $0.39 \mathrm{~ms}^{-1}$ \\
Wavelength $(L)$ & $7.5 \mathrm{~km}$ \\
Jet half-width $(\lambda)$ & $1 \mathrm{~km}$ \\
Phase speed $\left(c_{x}\right)$ & $0.046 \mathrm{~ms}^{-1}$ \\
Jet amplitude function $(B(t))$ & $A+\epsilon \cos (\omega t)$ \\
$A$ & $1.2 \mathrm{~km}$ \\
$\epsilon$ & $0.3 \mathrm{~km}$ \\
$\omega$ & $0.00015 \mathrm{~s}^{-1}$ \\
Time conversion factor $(\tau)$ & $2592 \mathrm{~s}$ \\
Distance conversion factor $(\xi)$ & $1 \mathrm{~km}$ \\
\hline
\end{tabular}

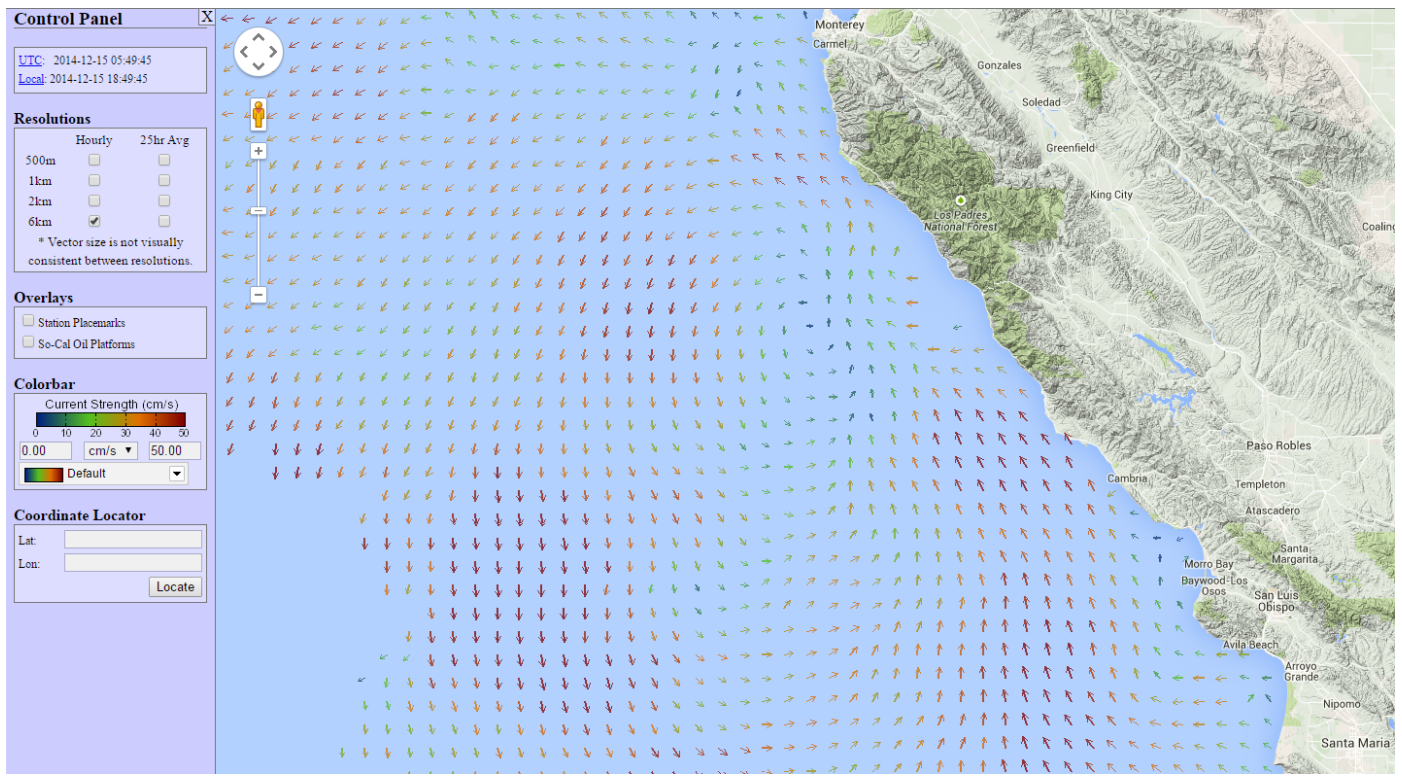

Figure 3.3: Hourly averaged surface current velocity field off the Californian Coast, obtained from high frequency (HF) radar measurements for UTC 2100, the 12th of December, 2014. Each arrow corresponds to a $6 \mathrm{~km} \times 6 \mathrm{~km}$ grid point. Image from [7]. This resource was specifically identified to the thesis author [142] by the developers of the MCM model [32] to clarify what the model represents in terms of coastal current, as where vortices and meandering jet-like structures present in the model could be seen.

jet were observed to be at the leading and trailing edges of meanders respectively.

A key insight made by Bower was to realize that this behaviour could be explained by modelling the current pattern as propagating. The jet pattern is itself moving 


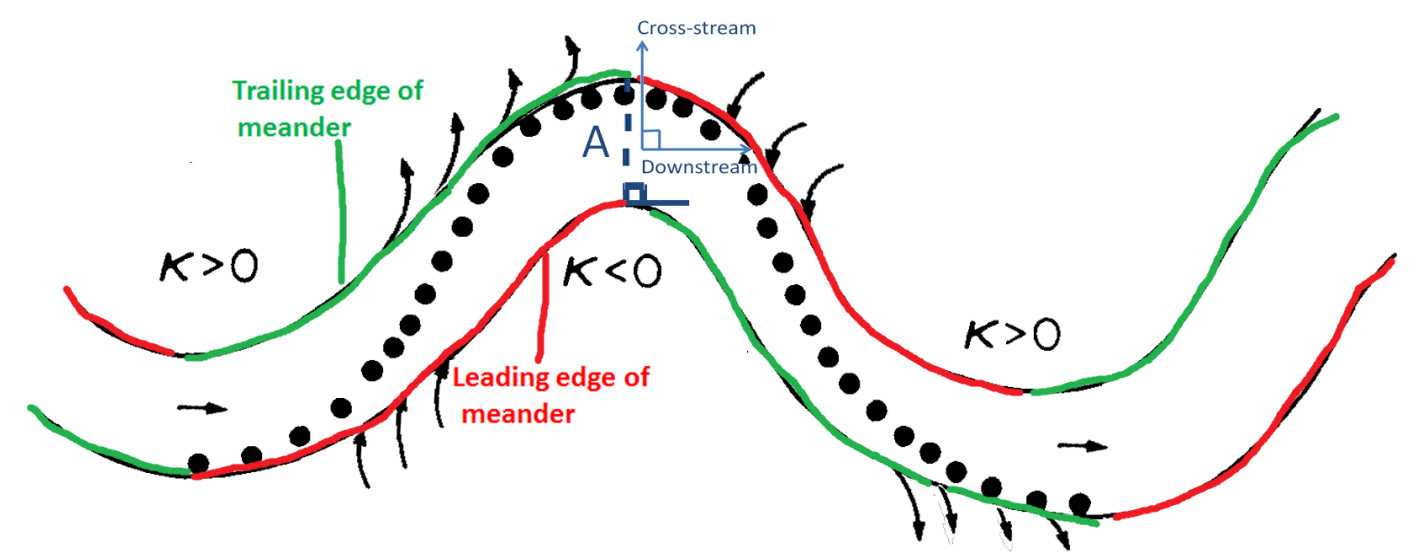

Figure 3.4: Schematic view of the horizontal motion of floats/fluid parcels in the meandering jet Gulf Stream current as observed by Bower [26]. The symbol $\kappa$ denotes the curvature of the meandering jet (bounding lines shown), and the dots correspond to a representative float trajectory moving towards the right. The dashed line (in blue) illustrates the orientation, which is normal to the jet, of the "stream" axis that forms the abscissa for graphs of mean current properties, such as Figure 3.7b). The arrows outside of the jet indicate the general pathways found by Bower where fluid parcels enter and leave the Gulf Stream. Figure modified from [26].

eastwards at a constant phase speed $c_{x}$ in the MCM model. A fluid parcel coming to a meander crest moves to the left of the jet pattern, since the jet pattern has travelled ahead of the parcel in this time. This results in fluid parcels near the leading edge of a meander traversing into a region of higher downstream speed and becoming further entrenched in the current, while fluid parcels near the trailing edge move into a region of lower downstream speed, due to the jet's velocity decreasing further from its centre. If in this region the zonal current velocity is less than $c_{x}$, the jet pattern will steadily move past the parcel and leave it behind. This results in the typical areas of detrainment and entrainment, and cross-stream movement behaviour that were observed. The vertical velocity of the MCM current is zero. This is consistent with the trajectories of the floats observed by Bower [27], where their vertical velocities were found to be negligible. Across all floats the mean vertical speed was around $0.08 \mathrm{cms}^{-1}$, compared to a horizontal speed of $67 \mathrm{cms}^{-1}$.

As identified by Samelson [165], the MCM current is divided up into three regimes in which different mobility behaviours are exhibited: a central jet, recirculating vortices and exterior retrograde relative motion. These are displayed in Figure 3.5. The central jet regime consists of the region near the centre of the jet where fluid parcels may experience some cross-stream movement, but not enough to displace the parcel out of the jet. Surrounding this area is the recirculating vortices regime where, at the outer edges of the jet, parcels' decreases in their eastward velocities at 
a meander crest allow them to be left behind by the pattern. These parcels, being outside of the current, remain stationary in the region frame until they are picked up by another meander crest passing over it at the same $y$ displacement. They then move in the direction of the jet until they are detrained at the next meander trough, and the process repeats. In the comoving frame moving at the eastward propagation speed of the meander pattern, the parcel appears to be travelling backward during the time that it is stationary in the world frame. This causes its path to appear as a closed circulation, with its trajectory within the jet being connected by a period of relative retrograde motion. Fluid parcels in the third regime are outside the current and hence stationary. In the comoving frame, this means they appear to be moving backwards.

No fluid exchange occurs between the regimes when the jet amplitude is constant. This constant amplitude was used in Bower's original formulation of MCM [25]. In fact, the movement of fluid parcels between regimes is commonly observed in the real-life current. For instance, Bower and Rossby [27] found that many of the floats they deployed near the centre of the jet (regime 1) escaped (entering regime 2) and were reabsorbed into the stream (returning to regime 1). Some mechanism for fluid exchange must exist. To introduce fluid exchange, Samelson [165] modified the amplitude of the meanders to be time-varying, so $B=B(t)=A+\epsilon \cos (\omega t)$.
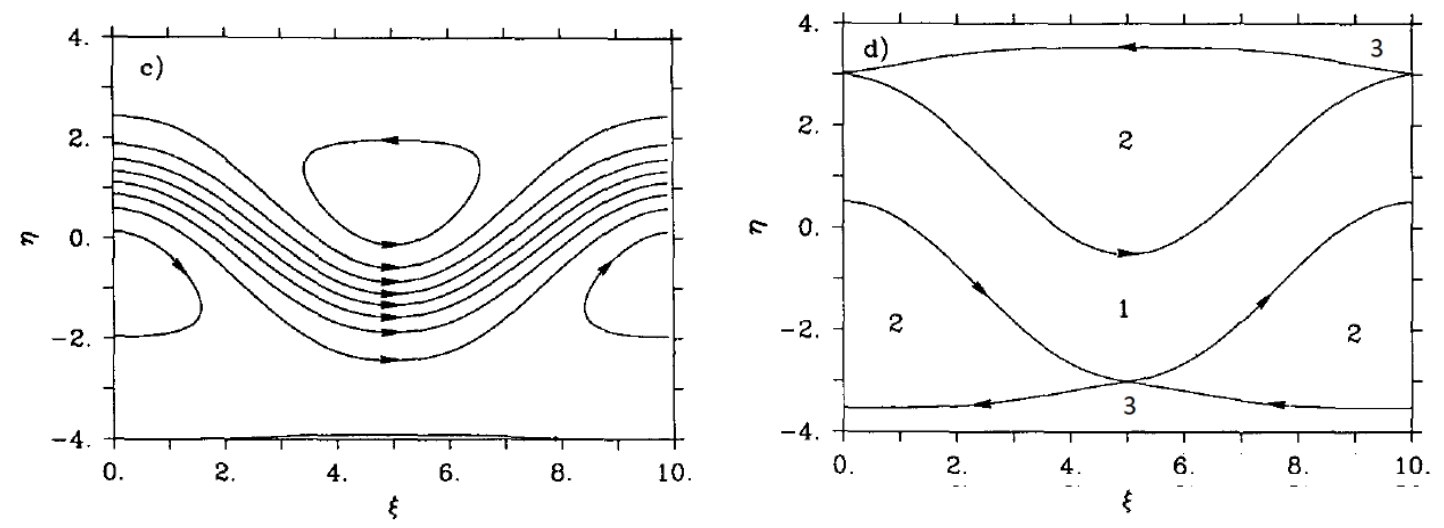

Figure 3.5: Illustration d) of MCM current regimes side-by-side with c) the MCM current, with both being depicted in the comoving frame of reference moving at the eastward propagation velocity of the current. In d), region 1 corresponds to the central jet regime, 2 to the recirculating vortices and 3 to the exterior retrograde relative motion, with the separatrices defining the boundaries between regimes. Subfigure c) plots the streamlines of the current. Streamlines are level curves of $\psi$. As the streamfunction is constant in the comoving frame, these streamlines correspond to the paths that fluid will take in this frame [144]. Image from Samelson [165]. 


\subsection{Previous 3D extensions of the MCM model}

As reviewed in the previous section, MCM is a current mobility model that is able to reproduce observed behaviours of the Gulf Stream current and with different scalings has also been proposed for coastal currents. The original MCM model only specifies the current in a 2D plane and thus is not useful for simulating UWSNs, such as of the form in Figure 1.1, that span the vertical as well. A number of UWSN current mobility models that extend MCM to 3D have since been developed. These models are surveyed in this section and their realism qualitatively assessed in Section 3.4.

Lee et al. [113] use the MCM model to evaluate the performance of the UWSN routing protocol they propose. A network deployed in a $1000 \mathrm{~m}$ deep box is simulated. The MCM model is extended to $3 \mathrm{D}$ by having the velocities at a $2 \mathrm{D}$ point, given by (3.2-3.4), also apply to all points above and below that point. Thus two nodes at different depths, but identical horizontal positions, have the same drift velocity. The streamfunction uses the parameters designated for coastal currents by Caruso et al. [32] based on the main jet speed being described as $0.3 \mathrm{~ms}^{-1}$. The model is not described in detail by Lee et al. [113]. However, in private correspondence with the authors [111], it was confirmed that the model used was the one stated above. The same 3D MCM model is also employed by Noh et al. [134].

Alvarez et al. [14] investigate reducing the energy utilized by an AUV in reaching in a destination, by having the device choose a path through areas where the current supports the vehicle in reaching its goal. For the evaluation of the path planning algorithm they propose, the authors develop a 3D version of Bower's current [27] as shown in Figure 3.6. The current is defined at three depths only, the AUV only being given these depth choices and is assumed to be able to transition between them instantaneously. The current at each layer is Bower's current, shifted horizontally according to differing offsets but otherwise remaining unchanged in strength or shape between layers. No justification is given for the 3D current being shifted. In any case, the model is not directly adaptable to use in simulating 3D UWSNs as the current is not defined continuously but only at discrete depths.

A criticism of MCM is that it is completely deterministic. The velocity is given by the derivative of the streamfunction, and for a fixed time and position the result will be the same from trial to trial. However, currents are also propelled by winds, which are ever-changing. Erol et al. [57] develop a 3D version of MCM named MCM-SE (Meandering Current Mobility with Surface Effects) that models the effect of the winds on currents near the surface by adding a stochastic component to the 

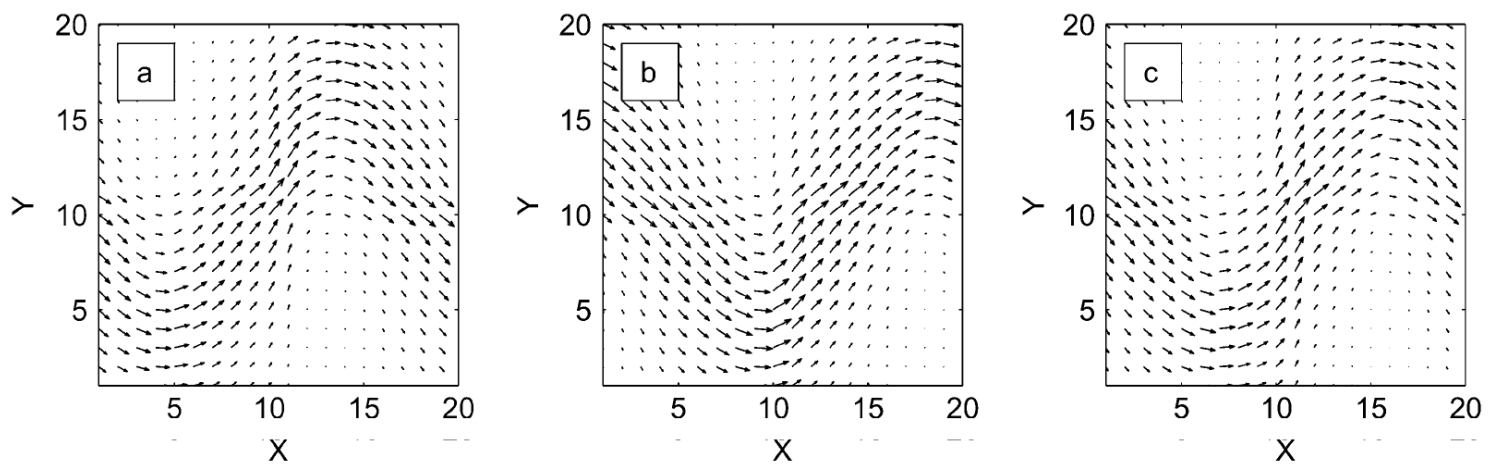

Figure 3.6: 3D extension of Bower's streamfunction [25] used as a current in [14]. In their scenario, the AUV can only move vertically to three layers, where the velocity field at each depth is the same compared to each other but shifted to the left or right. The figure shows the velocity field in for the (a) first (shallowest), (b) second and (c) third (deepest) layers at the same time instant. The depth in metres of each layer is not given and immaterial to the authors, as it is assumed that the AUV can move instantly to each layer. Image from [14].

underlying MCM velocity. Below $250 \mathrm{~m}$ depth, considered outside the influence of wind stress-induced currents, the velocity is given by the MCM model. As for [14] , the same 2D MCM flow is applied to each depth, with no rationale given for this choice. The underlying MCM flow is thus depth-invariant.

Above $250 \mathrm{~m}$ depth, an additional random velocity caused by the wind, $\left(u_{s}, v_{s}, 0\right)$, is added to that of the MCM model. This stochastic component also doesn't change with depth. The velocity, which in simulation is updated at discrete time intervals, is given by

$$
\begin{aligned}
& u_{s}(t+\Delta t)=u_{s}(t) e^{-\lambda \Delta t}+U \sqrt{1-e^{-2 \lambda \Delta t}} \zeta_{i} \\
& v_{s}(t+\Delta t)=v_{s}(t) e^{-\lambda \Delta t}+U \sqrt{1-e^{-2 \lambda \Delta t}} \xi_{i}
\end{aligned}
$$

where $\zeta_{i}$ and $\xi_{i}$ are independent pseudo-random numbers drawn from a zero-mean, unit-variance Gaussian distribution. In the equation In the equation, the new velocity depends partially on the old value. This is meant to reflect that winds do not vary wildly from instant. The same randomly generated velocity is applied to all nodes above $250 \mathrm{~m}$ depth to reflect that the same wind is blowing across the entire network. MCM-SE is employed by its authors to study the performance of localization protocols and node movement schemes [57, 59, 172]. 


\subsection{The case for a depth-decaying current}

The 3D versions of the MCM mobility model that have been published $[14,57,113,134]$ have currents whose strengths do not change with depth. This is likely to be unrealistic. The ocean environments that MCM is intended for, the Gulf Stream and coastal currents, tend to be depth-decaying.

Hydrographic measurements of the Gulf Stream current off Cape Hatteras, in the same location where Bower [27] deployed her floats and developed her model for, made by Halkin and Rossby between 1980 - 1983 [72] revealed a flow structure that decreased in velocity with increasing depth. Whereas the speed at the centre of the jet was around $2 \mathrm{~ms}^{-1}$ on the surface, by $1000 \mathrm{~m}$ depth the speed was reduced to approximately $0.2 \mathrm{~ms}^{-1}$. Such a depth range is not atypical for potential UWSNs, with Lee et al. [113] considering a $1000 \mathrm{~m}$ deep SEA Swarm and Yan and Shi [205] a $500 \mathrm{~m}$ deep network. In such a current, nodes at the top and bottom of the network would have markedly different rather than depth-invariant mobility behaviours. The mean current velocity recorded by Halkin and Rossby is reproduced in Figure 3.7. Results are presented in the stream axis oriented normal to the jet as shown in Figure 3.4. The symbol $\bar{u}$ denotes the current velocity in the cross-stream direction, which is parallel to the stream axis, and $\bar{v}$ the downstream current velocity, which is perpendicular to the stream axis.

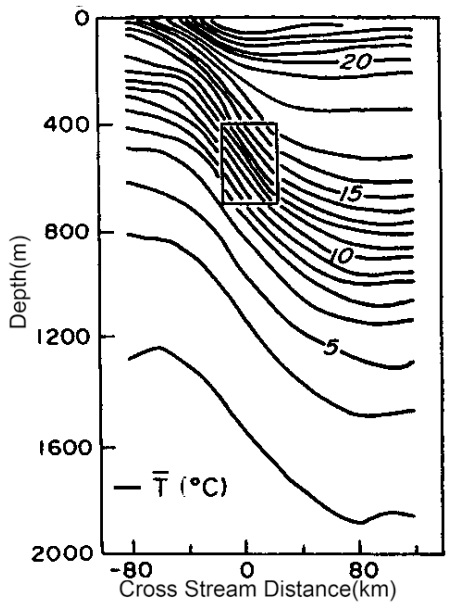

(a)

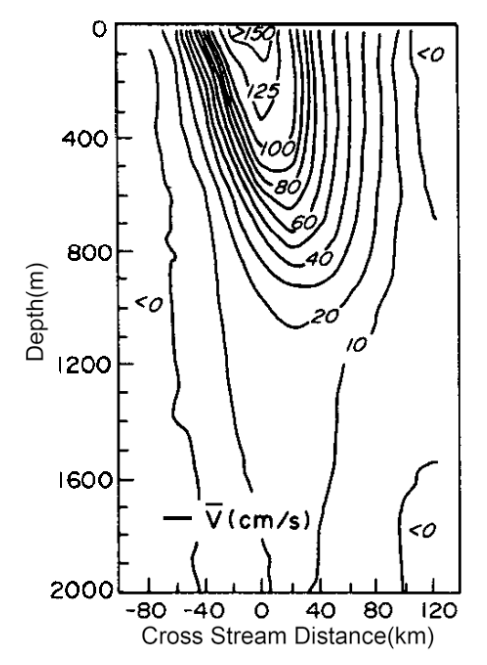

(b)

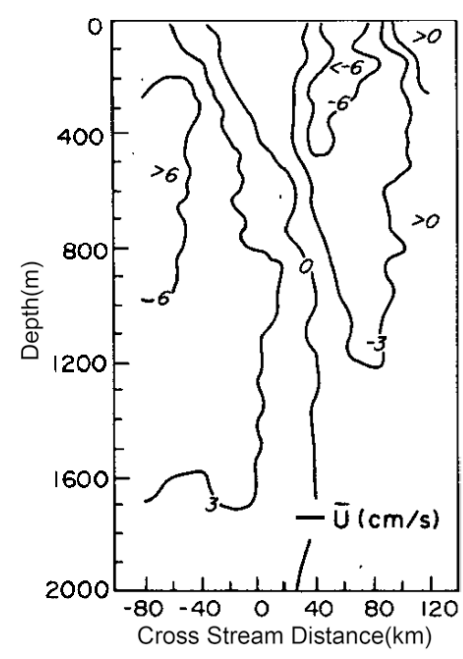

(c)

Figure 3.7: Mean a) temperature, b) downstream current velocity, c) cross-stream current velocity in the Gulf Stream at various depths and along the "stream" axis. Image from Halkin and Rossby [72]. 
Similarly, coastal currents typically fall off in intensity with depth, and in general, the speed of currents in the ocean tend to be depth-decaying [185, p. 165]. Some examples of this observed current behaviour in the literature are given. Dahl [42] randomly selected 100 location across the globe as the simulation locations for his path planning scheme, in effect conducting a worldwide sampling of ocean currents. Predicted ocean currents from the HYCOM (HYbrid Coordinate Ocean Model) database, an ocean forecast model, were obtained for each location. Dahl reported that currents tended to be weaker at lower depths and stronger near the surface. San Pedro Channel, a coastal environment off southern California, was chosen by Smith and Huynh [182] to validate their float buoyancy control algorithm. The authors noted that the current velocity forecast for the region decreased with depth, with the the velocity falling to near zero below $\sim 200 \mathrm{~m}$.

There are strong physical reasons for why currents, in particular the mean or underlying current, tend to decrease in speed with increasing depth. Two of the main components of the current velocity are Ekman currents and geostrophic currents, both of which are depth-decaying.

The wind exerts a force on the sea surface giving rise to motion of the sea surface. Movement is induced at deeper layers by this motion due to fluid friction. As water molecules from the higher layer collide with particles in the lower layer (molecular viscosity) and, more importantly underwater, macroscopic parcels of water are exchanged (eddy viscosity), they impart some of their momentum, on average accelerating the lower layer in the direction of the surface stratum [140, p. 40]. The flow that results from the blowing of a steady wind is known as the Ekman current and is shown in Figure 3.8. The flow typically extends to $10-200 \mathrm{~m}$ depth, dependent on the wind strength, in the mixed layer of the oceans $[152,186]$. In order for the current to be steady-state, the flow is directed at an angle so that the apparent Coriolis force affecting motion and wind stress force balance each other out.

The speed of the Ekman current decreases exponentially with depth as only a fraction of the kinetic energy of each layer is transferred to the layer below it [170]. Each molecule and fluid parcel is moving at the macroscopic velocity of the fluid plus a random, Brownian contribution. The mean velocity component causes the overall effect of collisions to speed up the slower layer. The stochastic component results in bodies striking each other at odd angles and causes the particles to careen off in random directions. Rather than adding to the overall directed motion, some of the energy is lost in increasing the energy of the random molecular motion of the fluid, otherwise known as heat, as it is transmitted downwards [64]. 


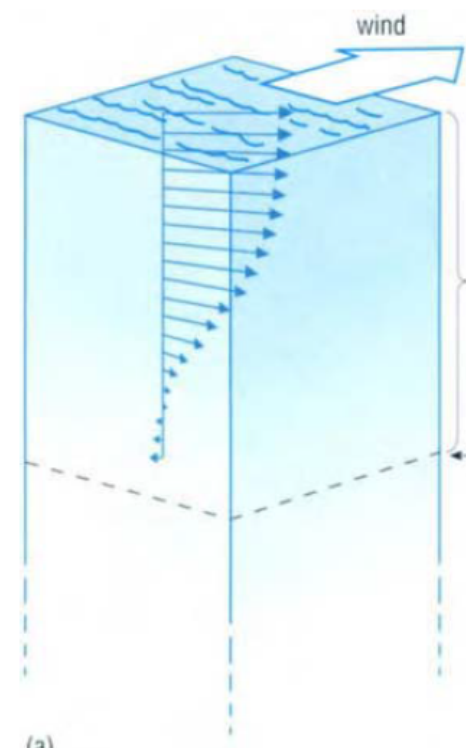

(a)

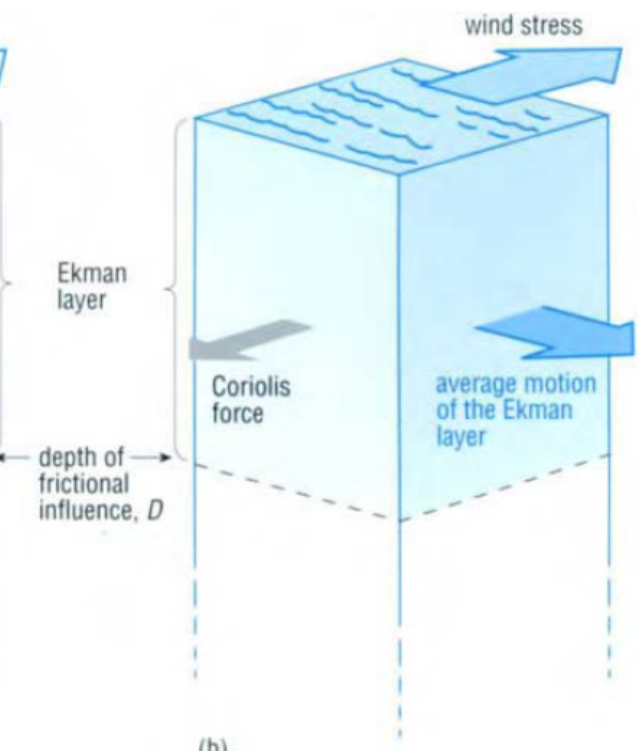

(b)

Figure 3.8: Theoretical Ekman current derived by consideration of the impact of viscosity and the Coriolis effect on an ideal ocean. Image from [140, p. 42]. The current a) is directed away and deviates further from the wind direction with depth, decreases in speed with depth and (b) the Coriolis and wind forces balance each other out.

The wind not only causes currents due to the direct actions of local winds. Because of persistent, large-scale wind systems that push and "pile up" water in areas, the oceans are not flat but at different heights [140, p. 92]. The sloping ocean surface sets up horizontal pressure gradient forces that produce geostrophic currents. Near the sea surface, the difference in pressure between the two ends of the sloping sea surface is primarily a consequence of the raised section of water. As one descends, the weights of the overlying columns of water must also be added to the pressure. The density of seawater is not constant with depth, and in most of the ocean it has been found that the water under the side with the lower sea surface increases in density more rapidly with depth [131, p. 135]. The more dense water compensates for the missing weight of the raised sea section, reducing the pressure gradient and associated geostrophic current with depth, as shown in Figure 3.9.

When the mean current over a day or more is calculated, it is the sum of the Ekman and geostrophic currents [152], being those driven by long-term processes. As both of these current components tend to decrease in strength with increasing depth, this leads to a mean current that is depth-decaying. The other current sources in the ocean operate at shorter time-scales and tend to zero out in the ensemble average. Tidal currents have a semi-diurnal or diurnal period. Inertial currents arise from 


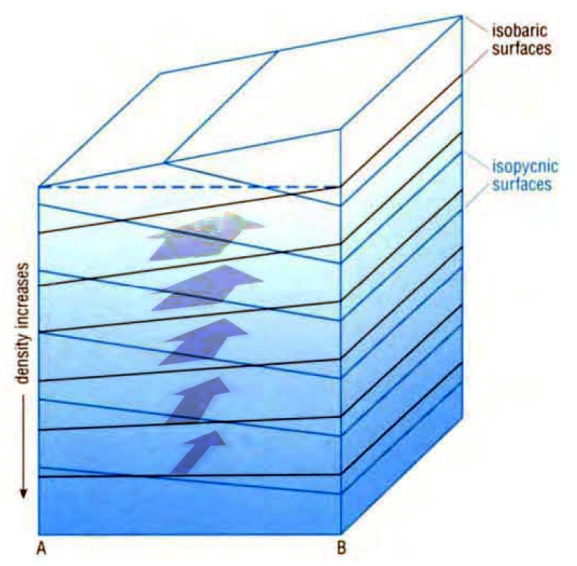

Figure 3.9: Decline in geostrophic current (grey arrows, speed expressed by the arrow width) associated with density variations in the ocean being inclined against the sea surface slope. Image from $[140]$.

the immediate value of the wind and motion due to the waves from the immediate value of the sea state, making them essentially impossible to predict and so they are typically modelled stochastically [56]. Observational data has found that these stochastic, high-frequency components have a zero mean. For more background on ocean currents refer to Appendix A.

\subsection{The velocity profile of the current mobility model}

We identified existing 3D MCM models as flawed in the last section. They defined the current at different vertical layers arbitrarily as depth-invariant, whereas the actual Gulf Stream and coastal currents being represented decrease in speed with increasing depth. In this work, we develop our own depth-decaying 3D extension of MCM that is more realistic by being based on measurements of the MCM current, the Gulf Stream.

In this section, we begin considering how real current data on the Gulf Stream can be integrated into the MCM model. A problem with MCM is that its parameters (3.1) only allow the shape, size and scale factor of the flow to be varied. Different ocean environments, such as coastal currents [32], have been modelled using MCM, where parameter values representative of that type of flow have been chosen. However, the choice of parameters does not affect the current's velocity profile, or how the velocity varies within the jet. This is an issue when the velocity profile of the real-life current 
differs significantly from the MCM current and could be more accurately represented by a modified profile. We derive in this section an expression, $U(r)$, for the velocity profile of the MCM current. Using this, a more representative velocity profile may be substituted in.

Consider the MCM streamfunction, (3.1). For a given $x$-value, the $y$-location of the centre of the jet is $y_{c}=B(t) \sin \left[k\left(x-c_{x} t\right)\right]$, and $\alpha=\tan ^{-1}\left\{B(t) k \cos \left[k\left(x-c_{x} t\right)\right]\right\}$ is the angle the centre of the jet makes with the positive $x$-direction. It is easy to recognize that $\alpha$ does represent the angle, since $\alpha=\tan ^{-1}\left(\partial y_{c} / \partial x\right)$.

Let us define an arbitrary point $\left[\begin{array}{ll}x & y\end{array}\right]^{T}$, as shown in Figure 3.10. Denote the distance of this point from the line tangent to the centre of the jet directly below the point, $\left[\begin{array}{ll}x & y_{c}\end{array}\right]^{T}$, as

$$
r(x, y, t)=\left(y-y_{c}(x, t)\right) \cos (\alpha(x, t))
$$

The value $r$ is approximately the distance of $\left[\begin{array}{ll}x & y\end{array}\right]^{T}$ from the jet along the crossstream axis. The approximation becomes more accurate where the curvature of the current is low and becomes equal to the cross-stream axis distance when the current is not curved $(\kappa=0)$.

In fact, it is impossible to uniquely determine the cross-stream axis associated with a point in the curved MCM current. The point may correspond to two different cross-stream distances on two axes at once, since the parallel curves of a sinusoid are not sinusoids themselves and the normals emanating from the centre intersect [4]. This is demonstrated in Figure 3.11 and is why we prefer using an approximation to describe the velocity profile.

The MCM streamfunction (3.1) can be expressed in terms of $r$ as

$$
\begin{aligned}
\psi(x, y, t) & =-\psi_{0} \tanh \left[\frac{y-y_{c}}{\lambda / \cos (\alpha)}\right] \\
& =-\psi_{0} \tanh \left[\frac{r}{\lambda}\right] \\
& =\mathcal{U}(r)
\end{aligned}
$$




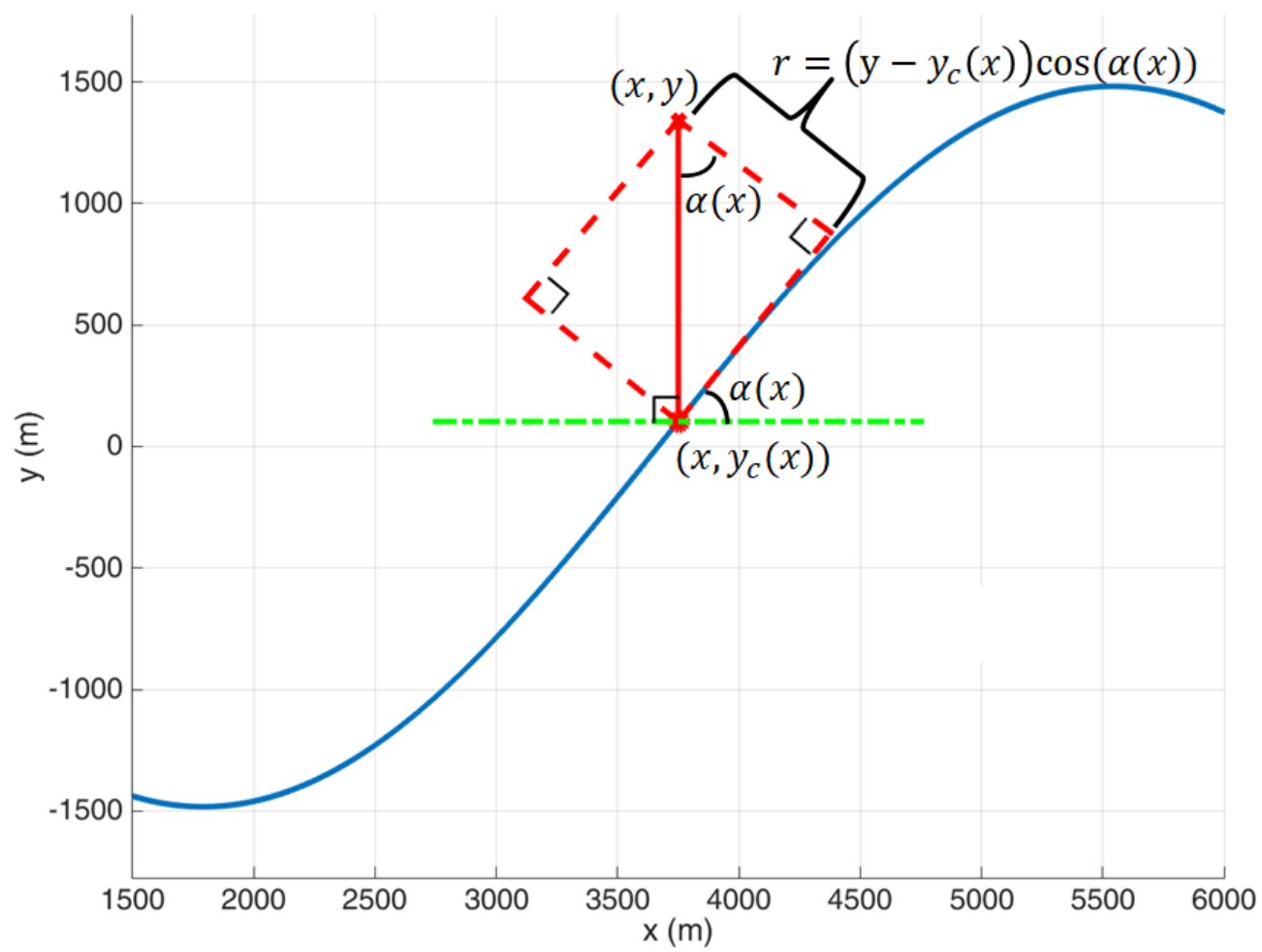

Figure 3.10: Physical significance of $r$ : distance of a point along the "approximate" cross-stream axis from the jet centre.
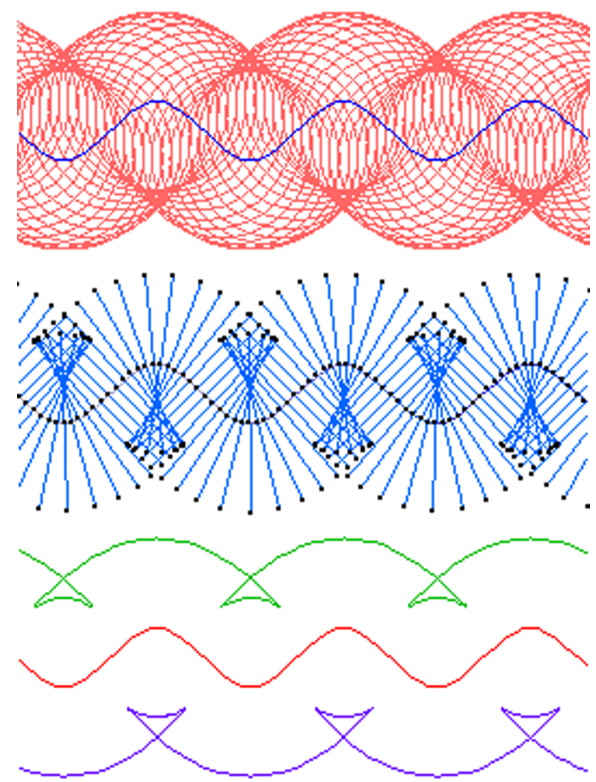

Figure 3.11: The curve parallel to a sine wave is not a sine wave. Image from [4] showing how the parallel curve of a sine wave is constructed from the locus of the end points of normal lines of the curve with fixed width. For the upper branch, near the meander troughs the normal endpoints reverse direction in the positive $x$-direction resulting in the wedge shaped loop. 
By the chain rule, the current velocities are given by

$$
\begin{aligned}
& u(r)=-\frac{\partial \psi}{\partial r} \frac{\partial r}{\partial y}=U(r) \cos (\alpha(x, t)) \\
& v(r)=\frac{\partial \psi}{\partial r} \frac{\partial r}{\partial x} \\
& =-U(r)\left(-\frac{\partial y_{c}}{\partial x} \cos (\alpha)+\left(y_{c}-y\right) \frac{\partial \alpha}{\partial x} \sin (\alpha)\right) \\
& =U(r)\left(\frac{\frac{\partial y_{c}}{\partial x}}{\sqrt{1+\left(\frac{\partial y_{c}}{\partial x}\right)^{2}}}+\frac{\partial^{2} y_{c}}{\partial x^{2}}\left(y-y_{c}\right) \cos ^{2} \alpha \sin \alpha\right) \\
& =U(r)\left(\sin (\alpha(x, t))+\frac{\partial^{2} y_{c}}{\partial x^{2}}\left(y-y_{c}\right) \cos ^{2} \alpha \sin \alpha\right) \\
& w(r)=0
\end{aligned}
$$

The expression for $v(r)$ comes about because of the trigonometric relationships [105, p. 811] $\cos \left(\tan ^{-1}\{x\}\right)=1 / \sqrt{1+x^{2}}, \frac{d}{d x}\left[\tan ^{-1}\{x\}\right]=\cos ^{2}\left(\tan ^{-1}\{x\}\right)$ and $\sin \left(\tan ^{-1}\{x\}\right)=$ $x / \sqrt{1+x^{2}}$.

The parameter

$$
U(r)=-\frac{d \mathcal{U}}{d r}=\frac{\psi_{0}}{\lambda} \operatorname{sech}^{2}\left[\frac{r}{\lambda}\right]
$$

The current speed at a point is

$$
\begin{aligned}
\|\mathbf{v}(r)\| & =\sqrt{[u(r)]^{2}+[v(r)]^{2}} \\
& =\sqrt{[U(r) \cos \alpha]^{2}+\left[U(r)\left(\sin \alpha+\frac{\partial^{2} y_{c}}{\partial x^{2}}\left(y-y_{c}\right) \cos ^{2} \alpha \sin \alpha\right)\right]^{2}} \\
& \approx \sqrt{[U(r)]^{2} \cos ^{2}(\alpha)+[U(r)]^{2} \sin ^{2}(\alpha)} \\
& =U(r)
\end{aligned}
$$

where the local curvature of the jet centre is only slowly varying, i.e., $\partial^{2} y_{c} / \partial x^{2}=0$. The value $r$ is the distance along the cross-stream axis from the jet centre when the current is not curved. Thus, the parameter $U(r)$ controls the jet velocity profile. The velocity profile is exactly $U(r)$ where the local curvature is zero, with there being a less exact correspondence in curvier sections of the current. The current velocity is in the downstream direction when the jet is not curved. Under those conditions, the angle between the jet centre and current is

$$
\cos \theta=\frac{U(r) \sin ^{2}(\alpha)+U(r) \cos ^{2}(\alpha)}{U(r)}=1
$$


so $\theta=0$ as expected.

The velocity profile utilized in MCM was given in (3.9). No explanation for why $\operatorname{sech}^{2}$ is used is provided by the authors [25,32]. It required extensive reading through the oceanographic literature to determine that the profile most lightly comes from an established relationship known as the Bickley jet. Bickley flows have been widely applied to the study of gaseous jets, mid-latitude atmospheric jets, oceanic thermocline jets, and the wake behind bluff bodies, to approximate the flow profile in such structures [188]. The Bickley profile is not based on surveys of the Gulf Stream, however, but comes from the exact solution found for the steady two-dimensional motion that arises from a jet issuing from a long narrow orifice into a fluid at rest. The ensuing flow is shown in Figure 3.12 where, far from the orifice in the $x$-direction, the velocity along the transverse axis is proportional to $\operatorname{sech}^{2}(y)$ and given by

$$
u=\left(\frac{3 M^{2}}{32 \nu x}\right)^{1 / 3} \operatorname{sech}^{2} \xi(x, y)
$$

where $\nu$ is the viscosity and $M$ is the constant momentum flux across any plane normal to the $x$-axis.

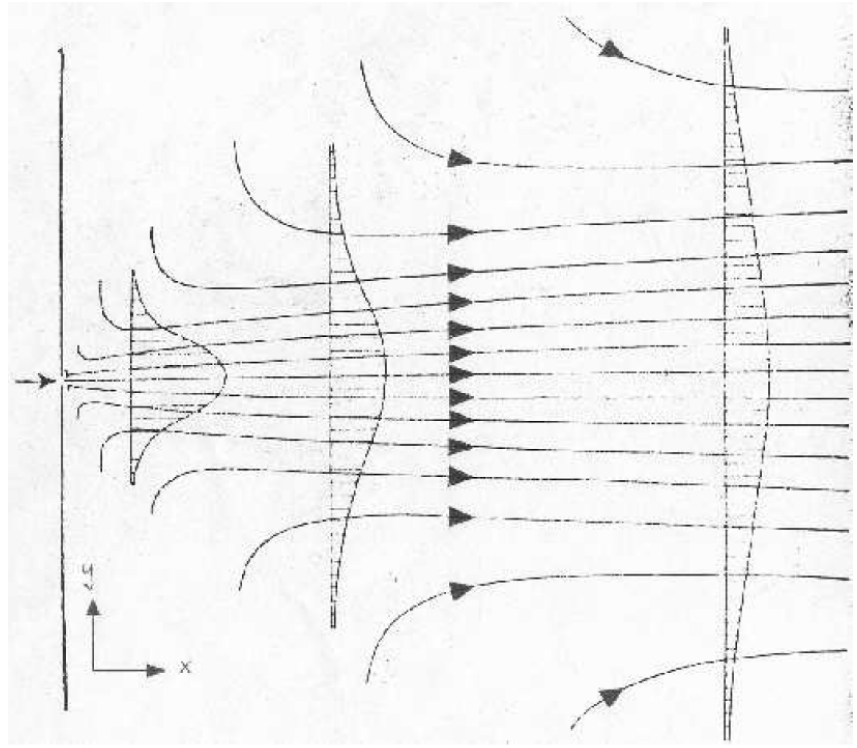

Figure 3.12: Streamlines and velocity profiles for the plane jet arising from a flow issuing from a long narrow orifice into a stationary fluid. Image from [23]. Far from the orifice, the flow is nearly parallel with the velocity profile along the $y$-axis being a function of $\operatorname{sech}^{2},|\mathbf{v}|=u=A \operatorname{sech}^{2}(B y)$. 


\subsection{A 3D Gulf Stream model}

Thus far, we have argued that existing 2D and 3D MCM models are unsuitable as representations of the 3D Gulf Stream. This is due to these models being depthinvariant, whereas the actual Gulf Stream is strongly depth-decaying, being 2D, and, as described in Section 3.5, having a jet profile based on a solution to an unrelated phenomenon. We present in this section our more realistic 3D MCM-based current mobility model for the Gulf Stream. The essence of our solution consists of using the measured average donwnstream velocity profile of the Gulf Stream diplayed in Figure $3.7 \mathrm{~b}$ as the value of the jet profile parameter, $U(r)$. Two models are developed: Current $A$ and Current $B$, a refinement of Current $A$.

The profile of the MCM current is near-constant all along the east-west length of the jet (see (3.10)), and in our proposed model is now given by Figure 3.7b. The prevalence of a single profile is consistent with the Gulf Stream's understood behaviour [80,84]. Furthermore, a separate experimental study by Bower and Rossby [27] has verified that the Halkin and Rossby mean profile applies over a large, continuous portion of the Gulf Stream. Bower and Rossby deployed 37 currentfollowing RAFOS floats into the Gulf Stream between 1984 and 1985. The floats' velocity data was used to investigate the structure of the current's velocity field. It was found that the mean downstream speed along the cross-stream axis was not significantly different from the mean speed in the profile obtained by Halkin and Rossby, and the velocity profile of the jet remained relatively constant throughout the regions measured by the floats. The authors considered this uniformity especially surprising as RAFOS measurements were made over an extensive range of downstream locations: from $34^{\circ} \mathrm{N}, 75^{\circ} \mathrm{W}$ to $40^{\circ} \mathrm{N}, 60^{\circ} \mathrm{W}$, or a distance of approximately $1500 \mathrm{~km}$. Figure 7 from their paper is displayed here as Figure 3.13 and shows how well Halkin and Rossby's profile describes Bower and Rossby's data.

Bower and Rossby's findings [27] conform to the view held by oceanographers that the flow in the Gulf Stream is relatively steady or constant. This is known as the "wiggly hose" or "rigid structure" view of the Gulf Stream [80,84]. It has arisen from studies that have found only a small fraction of the eddy kinetic energy of the current is due to changes in the velocity profile [73] and that the standard deviation of profile values is low compared to the mean over time [80].

We now discuss our two novel current models that utilize different approaches to reproduce Halkin and Rosbby's measured velocity profile in the model. 

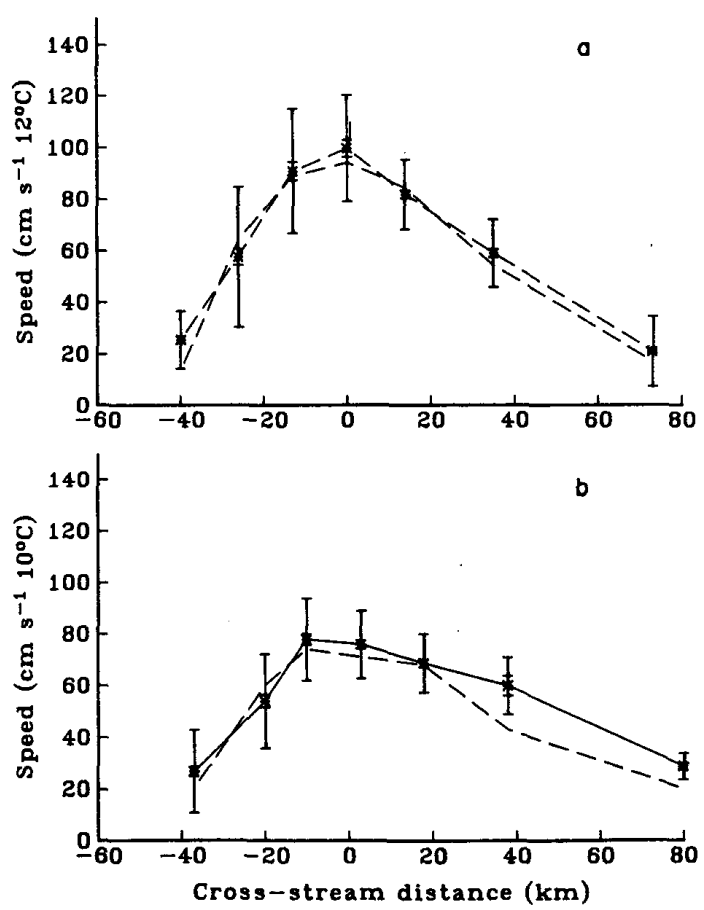

Figure 3.13: Mean downstream current speed in the Gulf Stream against cross-stream distance. The dashed line is based on measurements along a transect by Halkin and Rossby [72] in Figure 3.7b). The data for the solid line/marked points was obtained by floats deployed by Bower [25], showing close agreement with the transect data. (a) shows the speed on the $12^{\circ} \mathrm{C}$ isotherm, and (b) shows the speed on the $10^{\circ} \mathrm{C}$ surface. Bars indicate standard deviation (large bars) and standard error (small) of the mean. Figures from [26].

\section{Current A: depth-decaying scale factor}

Bower [27] originally represented 2D layers of the Gulf Stream at different depths with her model by varying the value of $s_{c}$, the maximum downstream speed in the centre of the jet. A typical speed at that depth was chosen. The values of the other parameters were kept unchanged. A direct extension of Bower's methodology to a full 3D current mobility model might be to define a continuous scaling function with depth for the scale factor, $\psi_{0}$, based on the current speeds measured in the Gulf Stream by Halkin and Rossby [72]. This is the approach taken in Current $A$.

The speeds in the centres of the jet, $s_{c}(z)$, were read off Figure $\left.3.7 \mathrm{~b}\right)$, as shown in Figure 3.14. The speeds were then divided by the velocity on the surface and interpolated to find the function for the normalization factor, $\psi_{N}(z)$, applied to the scale factor at each depth. The normalization factor is displayed in Figure 3.15. 


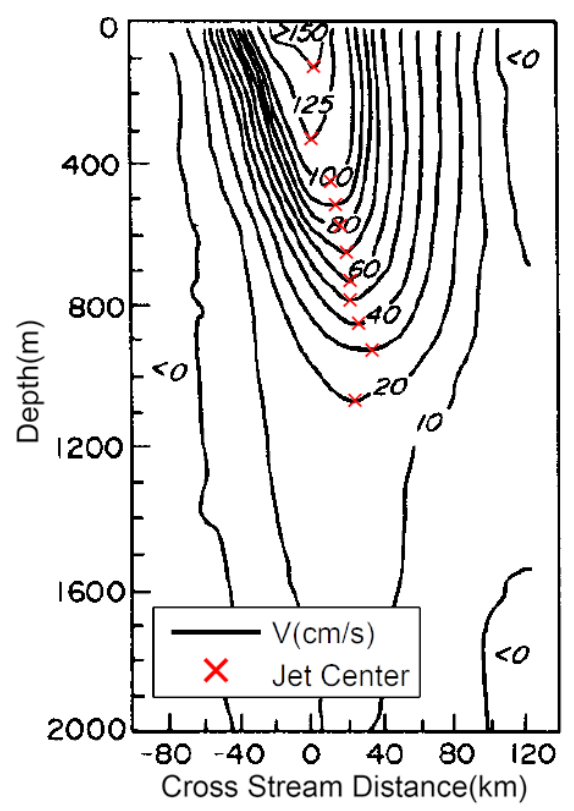

Figure 3.14: Jet centres identified in the average downstream velocity of the Gulf Stream $73^{\circ} \mathrm{W}$ measured by Halkin and Rossby [72]

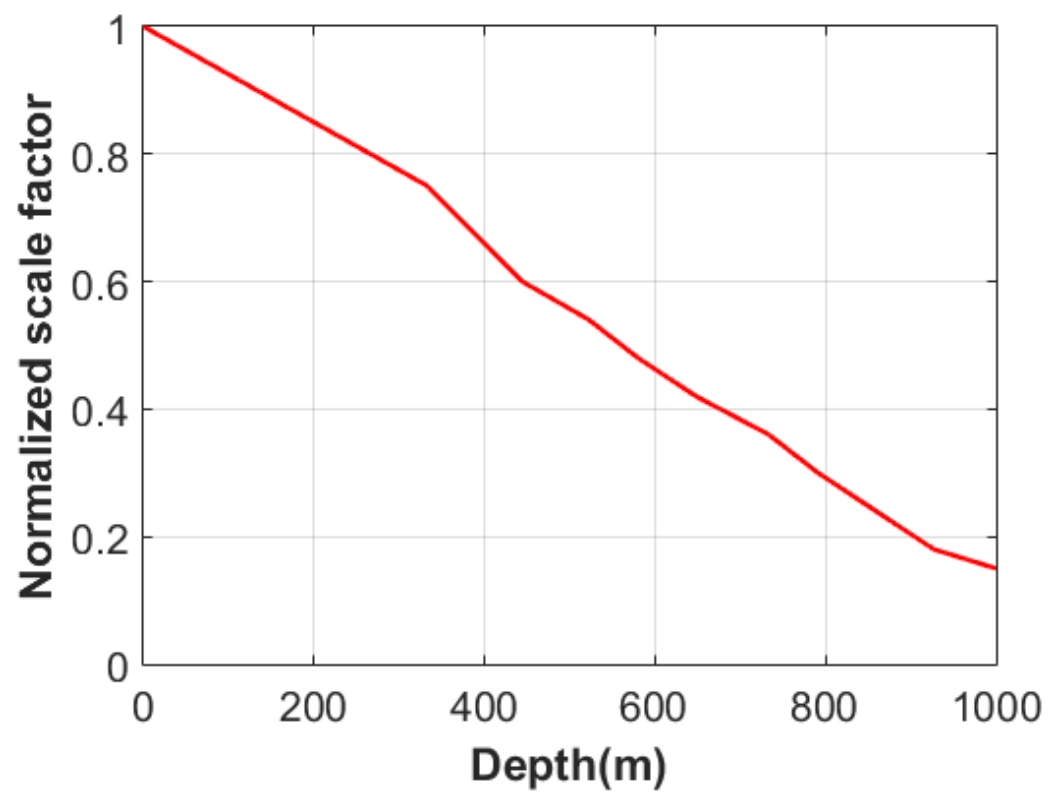

Figure 3.15: Normalization factor $\psi_{N}(z)$ for the scale factor $\psi_{0}(z)$ against depth

The scale factor is modified by the normalization factor according to

$$
\begin{aligned}
\psi_{0}(z) & =\lambda \psi_{N}(z) s_{c}(0) \\
& =\psi_{N}(z) \psi_{0}
\end{aligned}
$$

where $\psi_{0}=\lambda s_{c}(0)$ is the value of the scale factor on the surface. 
The flow in Current $A$ is now described by the function

$$
\psi(x, y, z, t)=-\psi_{N}(z) \psi_{0} \tanh \left[\frac{y-y_{c}}{\lambda / \cos (\alpha)}\right]
$$

where the current velocity is given by

$$
\begin{aligned}
u(x, y, z, t) & =-\frac{\partial \psi(x, y, z, t)}{\partial y} \\
v(x, y, z, t) & =\frac{\partial \psi(x, y, z, t)}{\partial x} \\
w(x, y, z, t) & =0
\end{aligned}
$$

As for Bower [25], all other parameters (defining the shape of the jet) besides the normalization factor remain constant with depth. The values of the other parameters are chosen to equal those used by Bower [25] to describe the surface Gulf Stream. Table 3.3 lists the values of the parameters in Current $A$.

Table 3.3: Current A parameter values

\begin{tabular}{lr}
\hline Parameter & Value \\
\hline Velocity profile, $U(r, z)$ & $\psi_{N}(z) s_{c}(0) \operatorname{sech}^{2}\left[\frac{r}{\lambda}\right]$ \\
Surface central downstream speed $\left(s_{c}(0)\right)$ & $2.31 \mathrm{~ms}^{-1}$ \\
Normalization factor $\left(\psi_{N}(z)\right)$ & As for Figure 3.15 \\
Wavelength $(L)$ & $400 \mathrm{~km}$ \\
Jet half-width $(\lambda)$ & $40 \mathrm{~km}$ \\
Phase speed $\left(c_{x}\right)$ & $0.12 \mathrm{~ms}^{-1}$ \\
Jet amplitude function $(B(t))$ & $B$ \\
$B$ & $50 \mathrm{~km}$ \\
\hline
\end{tabular}

\section{Current B: matched depth-decaying cross-stream current profile}

Current $A$ only changes the speed in the centre of the jet, not its location. The centre of the jet not only decreases in speed, but as seen in Figure 3.7b, is offset at different depths. To rectify this, in Current $B$ the velocity profile function, $U(r, z)$, is set to Figure $3.7 \mathrm{~b}$ so that all features of the real-life measured profile are captured. In adapting the plotted profile, the horizontal axis (cross-stream distance) corresponds to the values for $r$, and the vertical axis (depth) to the values for $-z$ ( $z$ being height). In reality, Figure 3.7b only gives incomplete information. To generate a $U$ that is 
defined at all cross-stream axis points, the value of $U$ at points not lying on contour lines were interpolated from the data in Figure 3.7b based on a triangulation-based linear scheme.

The current is now defined by a streamfunction

$$
\psi(x, y, z, t)=\mathcal{U}(r(x, y, t), z)
$$

where $r(x, y, t)=\left(y-y_{c}(x, t)\right) \cos (\alpha(x, t))$ and $U(r, z)=-\partial \mathcal{U} / \partial r$. The 3D velocity is given by

$$
\begin{aligned}
u(x, y, z, t) & =-\frac{\partial \psi}{\partial r} \frac{\partial r}{\partial y} \\
& =U(r(x, y, t), z) \cos \alpha \\
v(x, y, z, t) & =\frac{\partial \psi}{\partial r} \frac{\partial r}{\partial x} \\
& =U(r(x, y, t), z)\left(\sin \alpha+\frac{\partial^{2} y_{c}}{\partial x^{2}}\left(y-y_{c}\right) \cos ^{2} \alpha \sin \alpha\right), \\
w(x, y, z, t) & =0
\end{aligned}
$$

Table 3.4: Current B parameter values

\begin{tabular}{lr}
\hline Parameter & Value \\
\hline Wavelength $(L)$ & $400 \mathrm{~km}$ \\
Phase speed $\left(c_{x}\right)$ & $0.12 \mathrm{~ms}^{-1}$ \\
Jet amplitude function $(B(t))$ & $B$ \\
$B$ & $50 \mathrm{~km}$ \\
Velocity profile, $U(r, z)$ & Interpolated off Figure $3.7 \mathrm{~b}$ \\
\hline
\end{tabular}

The velocity profile of the jet in Current $B$ is, ignoring curvature effects, constant. In reality, while the Gulf Stream has what can be viewed as a "rigid structure" [80, 84] the cross-sectional flow profile of the western boundary current is still slightly fluctuating. Variability is typically represent in current mobility models by adding a stochastic component, such as random walk, to the overall fluid parcel/node velocity [37]. In random walk, a random velocity drawn from a Gaussian distribution is added to the velocity of each node. This random velocity is re-evaluated at fixed time intervals.

Measurements of the variance of the Gulf Stream profile velocity have been made, which show it to be around $25 \%[72,74]$. This provides a starting point for 
setting up the stochastic model. Ultimately, discretion was used and a random walk mobility model not incorporated into Current $B$ by us, because not all variables are known. In particular, it is unclear how frequently random walk should be updated as short-term variability was not measured in the variance studies. Halkin and Rossby [72] measured across the Gulf Stream at bimonthly intervals, with a mean and variance computed from the records over 3 years' worth of such measurements. The current was only assessed at one mooring in [74]. Usually, the random model is calibrated by comparing simulated dispersal with observed trajectories [37], however a survey program that might involve the deployment of drifters was outside of the scope of of the research grant.

The fear was that by inappropriately applying a random walk model, as many unrealistic mobility behaviours would be introduced as simply simulating nodes in the absence of this additional variability. The case of how Erol et al [57] represented wind stress-induced currents is used to illustrate this possibility. The authors extended MCM with wind stress-induced currents that were modelled as a global, randomly chosen velocity added to the overall velocity field above $250 \mathrm{~m}$ depth. This behaviour is likely to be unrealistic, as wind stress-induced currents are known to decrease in speed with depth rather than being constant. Kinetic energy from winds is transferred downwards as a result of friction between successive layers of the ocean. Each layer only transmits a fraction of its kinetic energy, with some energy lost as heat as well, so the amount of kinetic energy and the speed of the resulting current in each layer steadily decreases with depth [140,170, p. 40].

\subsection{A 3D coastal current model}

In the original MCM paper [32], Caruso et al. proposed using Bower's streamfunction (3.1) as a coastal current model by scaling the Gulf Stream-based streamfunction parameters. The coastal current values were obtained from the Gulf Stream-based streamfunction parameters defined by Cencini et al. [33] by multiplying horizontal distances by $\lambda_{\mathrm{GS}} / \lambda_{\mathrm{C}}$ and velocities by $v_{\mathrm{GS}} / v_{\mathrm{C}}$, where $\lambda_{\mathrm{GS}}=40 \mathrm{~km}$ and $v_{\mathrm{GS}}=$ $1 \mathrm{~ms}^{-1}$ are the half-width and maximum downstream velocities of the Gulf Stream respectively and $\lambda_{\mathrm{C}}=1 \mathrm{~km}$ and $v_{\mathrm{C}}=0.39 \mathrm{~ms}^{-1}$ are representative coastal current half-widths and downstream velocities chosen by Caruso et al.

We apply the same methodology to derive representations of coastal currents from our Gulf Stream models. Caruso et al.'s coastal current model parameters are assumed to be correct and used wherever possible, and other parameters are scaled 
by $\lambda_{\mathrm{GS}} / \lambda_{\mathrm{C}}$ and $v_{\mathrm{GS}} / v_{\mathrm{C}}$.

\section{Current 1: depth-decaying scale factor}

Current 1 is developed from Current $A$ by substituting in Caruso et al.'s [32] coastal current parameters (Table 3.2). The values employed for the parameters are given in Table 2 .

Table 3.5: Current 1 parameter values

\begin{tabular}{lr}
\hline Parameter & Value \\
\hline Velocity profile, $U(r, z)$ & $\psi_{N}(z) s_{c}(0) \operatorname{sech}^{2}\left[\frac{r}{\lambda}\right]$ \\
Surface central downstream speed $\left(s_{c}(0)\right)$ & $0.39 \mathrm{~ms}^{-1}$ \\
Normalization factor $\left(\psi_{N}(z)\right)$ & As for Figure 3.15 \\
Wavelength $(L)$ & $7.5 \mathrm{~km}$ \\
Jet half-width $(\lambda)$ & $1 \mathrm{~km}$ \\
Phase speed $\left(c_{x}\right)$ & $0.046 \mathrm{~ms}^{-1}$ \\
Jet amplitude function $(B(t))$ & $A+\epsilon \cos (\omega t)$ \\
$A$ & $1.2 \mathrm{~km}$ \\
$\epsilon$ & $0.3 \mathrm{~km}$ \\
$\omega$ & $0.00015 \mathrm{~s}^{-1}$ \\
\hline
\end{tabular}

\section{Current 2: matched depth-decaying cross-stream current profile}

In addition to utilizing Caruso et al.'s [32] coastal current parameters, the profile function in Current 2 is scaled. The peak speed of Caruso et al.'s coastal current is $0.046 \mathrm{~ms}^{-1}$ and the jet half-width is $1000 \mathrm{~m}$. By visual inspection of Figure 3.7b, we find that these values are approximately $1.6 \mathrm{~ms}^{-1}$ and $60 \mathrm{~km}$ respectively in the Gulf Stream current profile. Applying the horizontal displacement and velocity scaling to the Gulf Stream profile so that its peak speed and half-width matches Caruso et al.'s [32] current, the flow profile in use for Current 2 is expressed by

$$
\begin{aligned}
U(x, y, z, t) & =\frac{c_{x}}{1.6 \mathrm{~ms}^{-1}} \widetilde{U}\left(r\left(\frac{120 \mathrm{~km}}{\lambda} x, \frac{60 \mathrm{~km}}{\lambda} y, t\right), z\right) \\
& =0.029 \widetilde{U}(r(60 x, 60 y, t), z)
\end{aligned}
$$

where $\widetilde{U}(r, z)$ is given by Figure 3.7b. Table 3.6 lists the values of the parameters in Current 2. 
Table 3.6: Current 2 parameter values

\begin{tabular}{lr}
\hline Parameter & Value \\
\hline Wavelength $(L)$ & $7.5 \mathrm{~km}$ \\
Phase speed $\left(c_{x}\right)$ & $0.046 \mathrm{~ms}^{-1}$ \\
Jet amplitude function $(B(t))$ & $A+\epsilon \cos (\omega t)$ \\
$A$ & $1.2 \mathrm{~km}$ \\
Velocity profile, $U(r, z)$ & $0.029 \widetilde{U}(r(60 x, 60 y, t), z)$ \\
$\widetilde{U}(r, z)$ & Interpolated off Figure $3.7 \mathrm{~b}$ \\
\hline
\end{tabular}

Figure 3.16 displays the downstream and cross-stream current velocities at two positions. The velocities have been overlaid on top of the Halkin and Rossby data [72], which have been similarly scaled according to (3.18). At $\left[\begin{array}{lll}x & y & z\end{array}\right]^{T}=$ $\left[\begin{array}{lll}1875 & 1500 & 0\end{array}\right]^{T}$ the jet centre is horizontal so by $(3.10), \sin (\alpha)=0$ and $\cos (\alpha)=1$, $u(x, y, z, t)=U(r(x, y, t), z)$ and $v(x, y, z, t)=0$. The downstream current should then match the fixed flow profile exactly, and this is what is broadly observed (the slight differences are due to interpolation artefacts from scanning in the measured velocities). The current across the jet centre at $\left[\begin{array}{lll}x & y & z\end{array}\right]^{T}=\left[\begin{array}{lll}4500 & -882 & 0\end{array}\right]^{T}$ deviates slightly from the measured profile, with deviation depending on the curvature of the centre. Cross-stream velocities remain negligible throughout the current.

It is questionable whether scaling of a Gulf Stream model can result in an accurate coastal current model, which casts doubt on [32]. The Gulf Stream and coastal currents arise from completely different ocean processes, and so may not be adequately represented by the same model. The Gulf Stream is a fast-flowing, primarily geostrophic current set up by the varying height of the sea surface [140, p. 85], which slopes downwards from the Western Atlantic Ocean towards the East Coast of the United States. Measurements by Halkin and Rossby [72] of the Gulf Stream current found the effects of tidal and inertial currents to be minimal when compared with the overall speed of the current on the surface of around $2.31 \mathrm{~ms}^{-1}$, with the speed of tidal currents less than $0.012 \mathrm{~ms}^{-1} \mathrm{rms}$ and inertial currents $0.035 \mathrm{~ms}^{-1} \mathrm{rms}$. Coastal currents on the other hand are typically dominated by tidal currents [133]. Rather than making up a small proportion of the overall geostrophicdominated current velocity, the velocity of the tidal component is often comparable to the geostrophic component. In measurements of the current $15 \mathrm{~km}$ off Cape Cod, Massachusetts in [177], the amplitude of the tidal current was found to be $0.3 \mathrm{~ms}^{-1}$, 


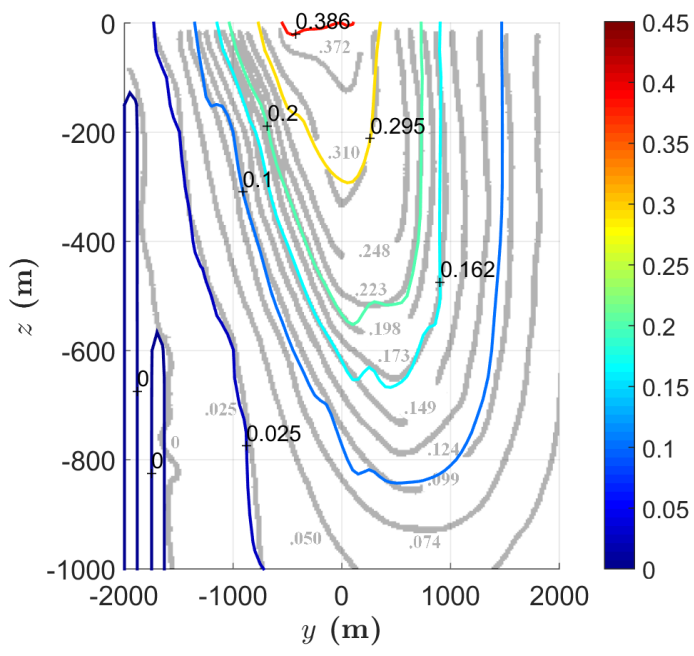

(a)

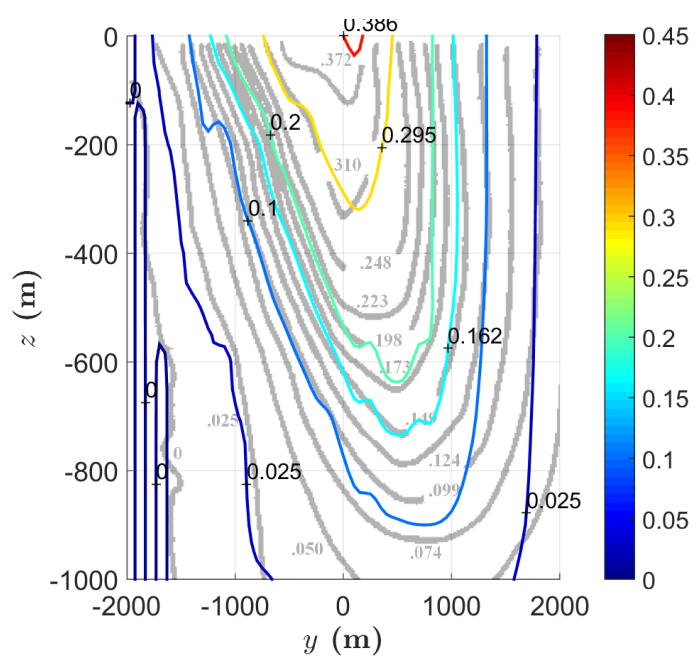

(c)

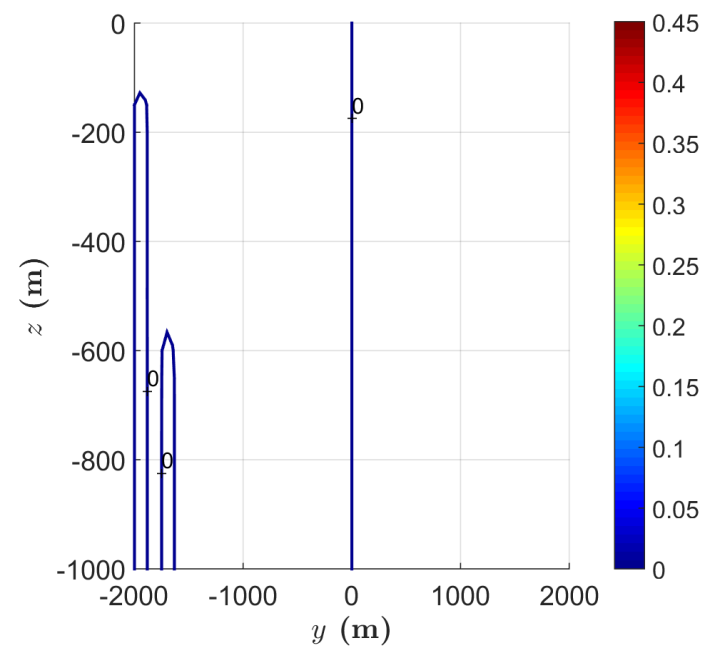

(b)

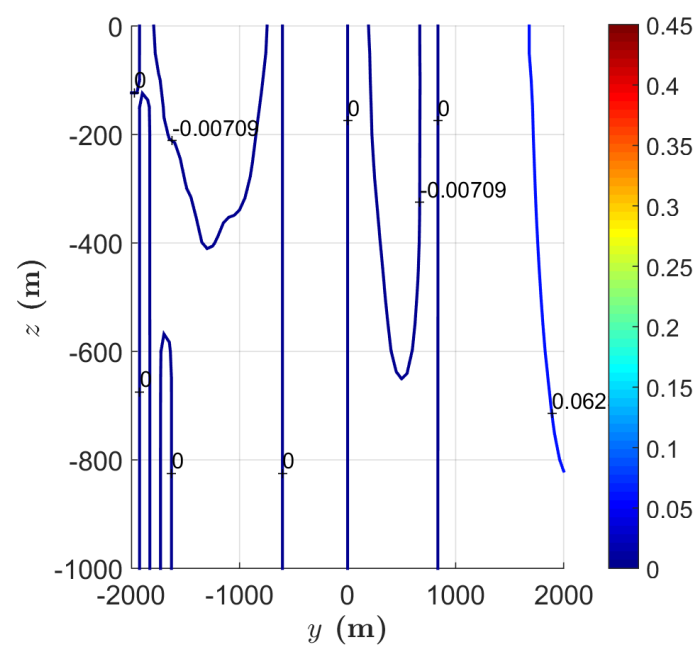

(d)

Figure 3.16: Current 2: a) downstream and b) cross-stream current velocity along the normal to the jet centre at $(1875,1500,0)$, and c) downstream and d) cross-stream current velocity along the normal to the jet centre at $(4500,-882,0)$.

while the geostrophic velocity was typically on the order of $0.2 \mathrm{~ms}^{-1}$. With the terms no longer being negligible in coastal currents, the tidal and other high-frequency current components need to be represented in the geostrophic-derived flow described by MCM.

The realistic incorporation of tidal and inertial efforts into MCM, so that it more closely resembles a coastal current it is claiming to represent, is an interesting topic for future work. This would also address the criticism that MCM is deterministic. 


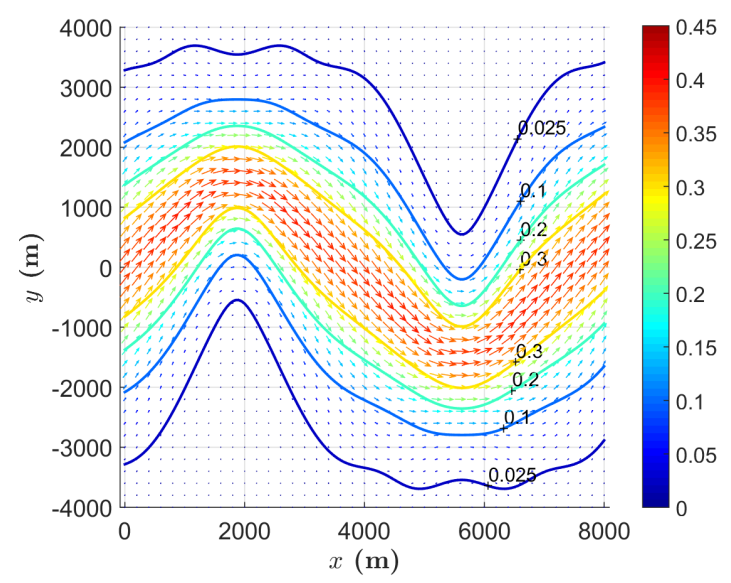

(a) Current $1, z=0 \mathrm{~m}$

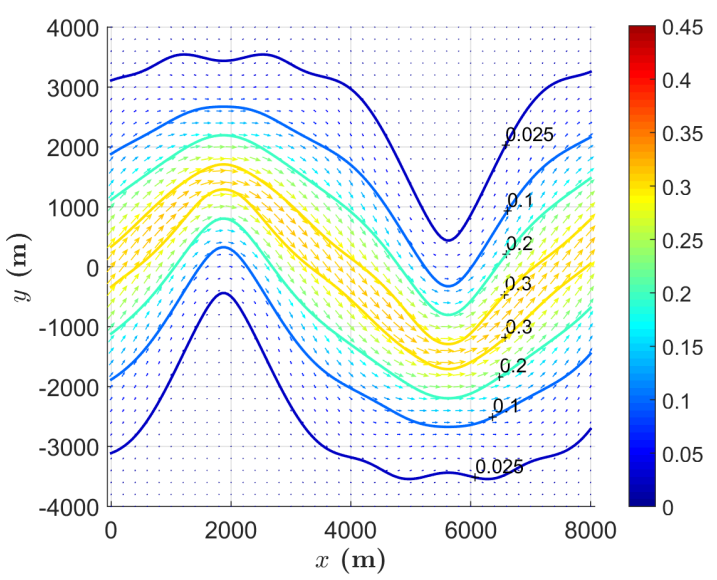

(c) Current 1, z=-250 m

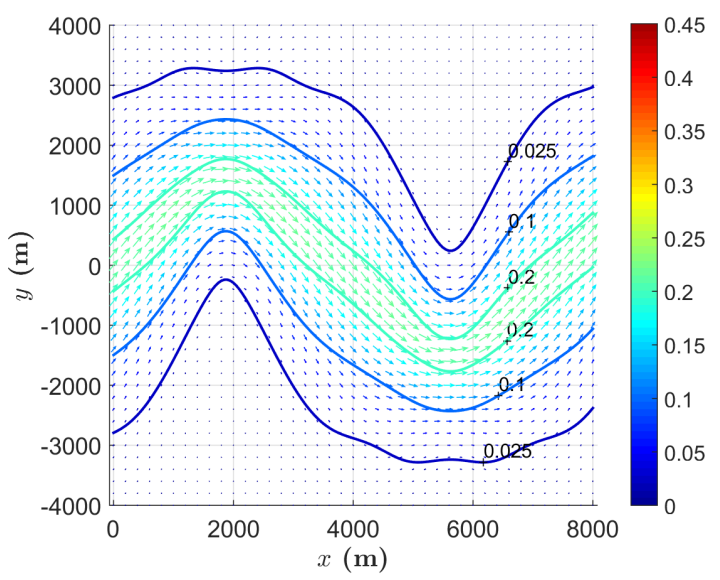

(e) Current 1, $z=-500 \mathrm{~m}$

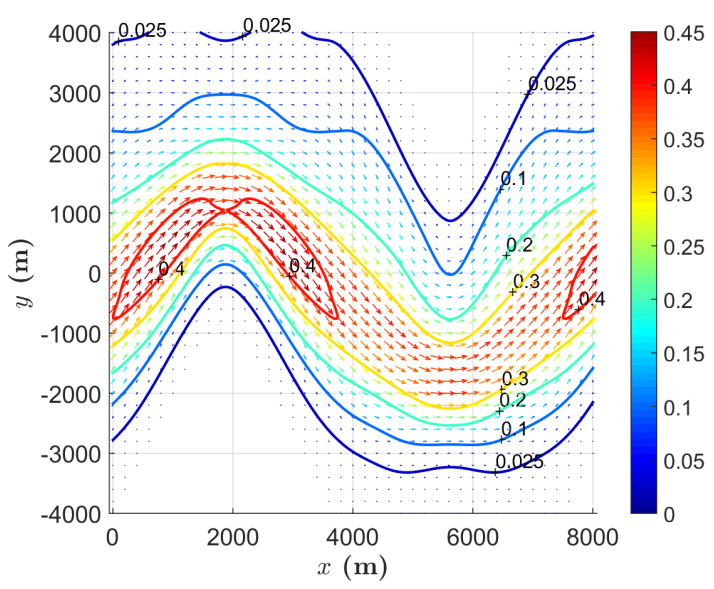

(b) Current 2, $z=0 \mathrm{~m}$

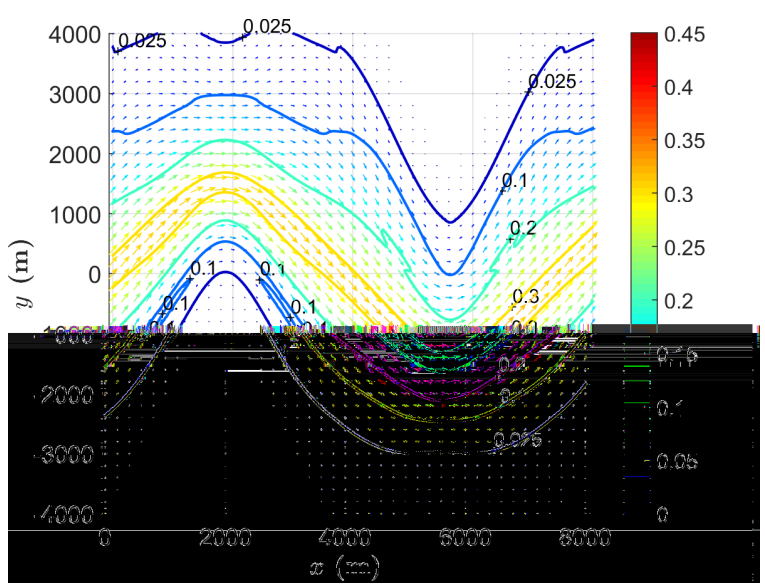

(d) Current 2, $z=-250 \mathrm{~m}$

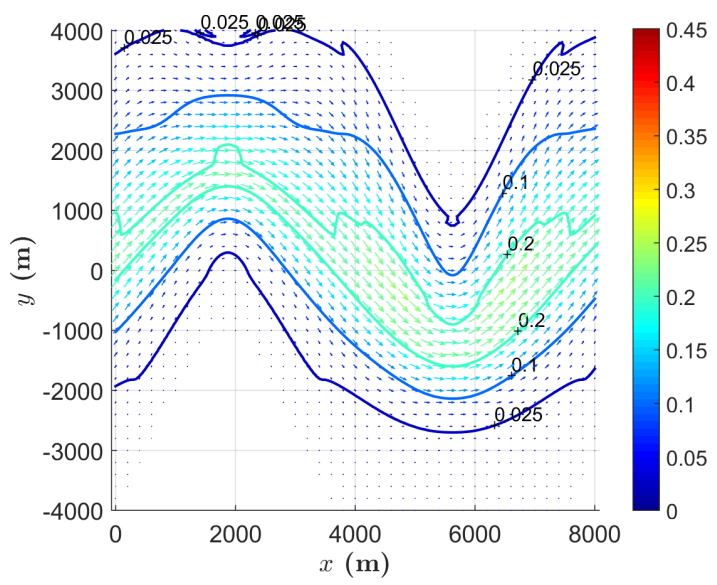

(f) Current 2, z=-500 m

Figure 3.17: Current velocity field in the region frame of reference of Current 1 at $t=0$ along heights a) $z=0 \mathrm{~m}$, c) $-250 \mathrm{~m}$, e) $-500 \mathrm{~m}$, and of Current 2 at $t=0$ along heights b) $z=0 \mathrm{~m}, \mathrm{~d}$ ) $-250 \mathrm{~m}, \mathrm{f})-500 \mathrm{~m}$. 


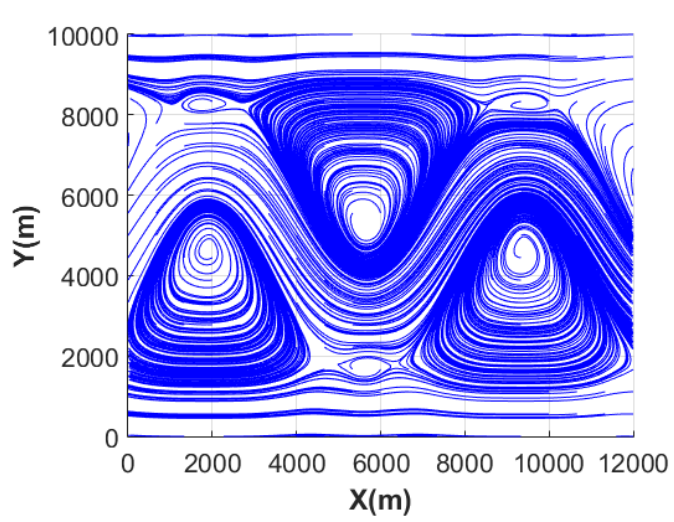

(a)

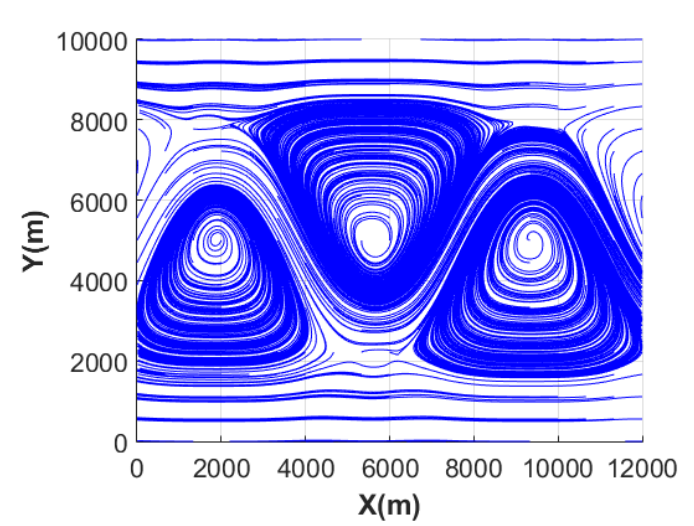

(b)

Figure 3.18: Current 1 mobility model streamlines in the comoving frame of reference at $t=0 \mathrm{~s}$ at (a)depth $=0 \mathrm{~m}, s_{c}=0.39 \mathrm{~ms}^{-1}$ and (b)depth $=500 \mathrm{~m}, s_{c}=0.22 \mathrm{~ms}^{-1}$.

In other works, tidal currents have been represented by the model [20]

$$
\begin{aligned}
& u=0 \\
& v=K \cos 2 \pi \kappa t
\end{aligned}
$$

and high-frequency components by a stochastic velocity term [37,57]. The velocities in (3.19) are added on top of the speeds resulting from other effects and are defined for a domain where the shoreline is towards the north. The model adds a global velocity component that alternates being being directed to (tidal flood currents $[136,138]$ ) and away (ebb currents) from the coastline, where the period of the oscillation coincides with the tidal period.

Velocity fields for Currents 1 and 2 at different depths are displayed in Figure 3.17, and comoving frame streamlines for Current 1 are drawn in Figure 3.18. The graphs show two of the behaviours that our depth-decaying mobility models exhibit that are novel compared to existing depth-invariant 3D MCM models. Firstly, the current velocity decreases with depth. Secondly, the recirculating vortices (see Figure 3.5) penetrate deeper into the jet at lower levels. This means that fluid parcels leave and are reabsorbed into the jet over a greater area, which matches the increase in fluid exchange with greater depth reported experimentally in the Gulf Stream [25].

\subsection{Summary}

In this chapter, we proposed physically-inspired 3D mobility models for the Gulf Stream and coastal currents based on the MCM (Meandering Current Mobility) 
model [32], a 2D mobility model. This work was motivated by the lack of other realistic 3D mobility models. Existing 3D extensions of MCM, which were surveyed in this chapter, have arbitrarily assumed currents that have constant strength with depth. In this chapter, we showed that this assumption is not generally applicable to ocean currents based on a consideration of the physics inside the ocean; and this assumption definitely does not apply to the Gulf Stream based on past hydrographic surveys showing the current to be depth-decaying. Ocean currents typically decrease in strength with increasing depth.

The depth-decaying 3D extension of the MCM model that we developed is a more realistic representation of the Gulf Stream. Our solution fits the "rigid structure" theory of the Gulf Stream and replaces the Bickley jet velocity profile of the current with a measured profile, which matches the speed of the Gulf Stream past Cape Hatteras [27]. Based on the increase in the size of the vortex regions of our current with descending depth, lower down UWSN nodes will have a greater tendency to be detrained from the current. This in real life observed behaviour [27] is not present in past 3D MCM models, due to them being depth-invariant.

The ocean environments represented by our model are of particular significance to network researchers, being where UWSN pollution monitoring will likely be located, and our models allows for the mobility behaviour of network protocols in these regions to be more accurately evaluated. Harmful algal blooms form after eutrophication caused by sewage and typically appear off the coast of built-up areas [75], such as the South California Bight [179]. The waters off Texas and Louisiana are one of the major petroleum-producing areas in the US [9] and feed into the Gulf Stream. Various measures were recommended to incorporate additional variability and the contributions of inertial and tidal effects in the current. The tuning of these effects, such as the duration of random walks, to match observed values so they can be incorporated in the overall model, is a suitable subject for future work. 


\section{Chapter 4}

\section{Performance of pressure routing in drifting 3D underwater sensor networks for oil plume monitoring}

UWSN research has been hampered by a dearth of suitable 3D current mobility models. As surveyed in Chapters 1 and 3, velocities specified by numerical ocean models are too coarsely spaced apart to resolve densely-deployed sensor networks; underwater current mobility differs significantly from land-based mobility and hence is not represented accurately by terrestrial models such as random walk; and, while a physically-inspired meandering current mobility model (MCM) has been developed for 2D, 3D extensions of it are unrealistic due to being depth-invariant. The corollary of there being few realistic 3D underwater mobility models is that we, the research community, have very little idea how many published UWSN network schemes will perform in actual deployment. This prevents network operators from effectively configuring their systems with the appropriate protocol for the task.

Pressure routing is a recent category of geographic routing protocols that holds considerable promise for underwater networking applications. Pressure routing is less bandwidth-intensive, due to forwarding being restricted (to nodes based on depth). An issue is that two of the main pressure routing protocols that have been developed, Depth Based Routing (DBR) [205] and HydroCast [113], have only been assessed in simulation using unrealistic stochastic current mobility models and a debunked depth-invariant current mobility model.

In this chapter, we evaluate the performance of DBR and HydroCast in a simulated pollution monitoring scenario, where sensed data from a subsurface pollution layer needs to be delivered by these routing protocols. The current advecting the nodes in 
the area is given by our more realistic depth-decaying 3D coastal current model that we developed in Chapter 3 (Current 1). The impact drift has on routing protocol performance is assessed by the trend in packet delivery ratio, end-to-end delay and energy consumption over time, and the impact drift has on the network topology is measured by the connectivity.

Our work has two main contributions. One, this is the first time that DBR and HydroCast have been studied in a relatively accurate 3D current mobility model. The results in this chapter thus provide a clearer indication of how these routing protocols will perform in real-life deployments. Secondly, past studies have only examined the averaged performance over the entire duration of the scenario. This is the first work to quantify the performance of the network at different periods during the simulation. In our results, we found that routing protocol performance decreased rapidly, with the packet delivery ratio of DBR falling by $96 \%$ after 3 hours with 600 nodes deployed. HydroCast was less negatively affected by current drift due to the use of void recovery flooding.

This chapter is structured as follows. Section 4.1 introduces pressure routing and the two pressure protocols being evaluated, DBR and HydroCast. Past performance studies of these protocols are discussed in Section 4.2 to highlight the novelty of our simulation in realistic current conditions. The design of our simulation study is detailed in Section 4.3. Specifics of the parameters and models used are described sufficiently for reproducibility of the results. This includes implementation of current mobility in the QualNet simulator. The simulation results are presented and discussed in Section 4.4 .

\subsection{Pressure routing}

A drifting underwater wireless sensor network is a scalable ad-hoc network made up of low-cost sensor nodes that are deployed to collaboratively monitor a region $[41,206]$. The topology of a drifting 3D UWSN is shown in Figure 4.1. Each sensor node is untethered and equipped with a buoyancy-adjusting oil bladder system to dive to their required depth in the network and to surface for recovery and reuse [113]. Once at this depth, the node senses the surrounding environment while drifting with the currents. Sensed data is sent to special sensor nodes known as sinks, which act as gateways, and deliver the data to end-users at a monitoring station [11].

By an ad-hoc network, it is meant that each node also acts as a router: forwarding on packets sent by other nodes to their destination [145]. The sequence of nodes 


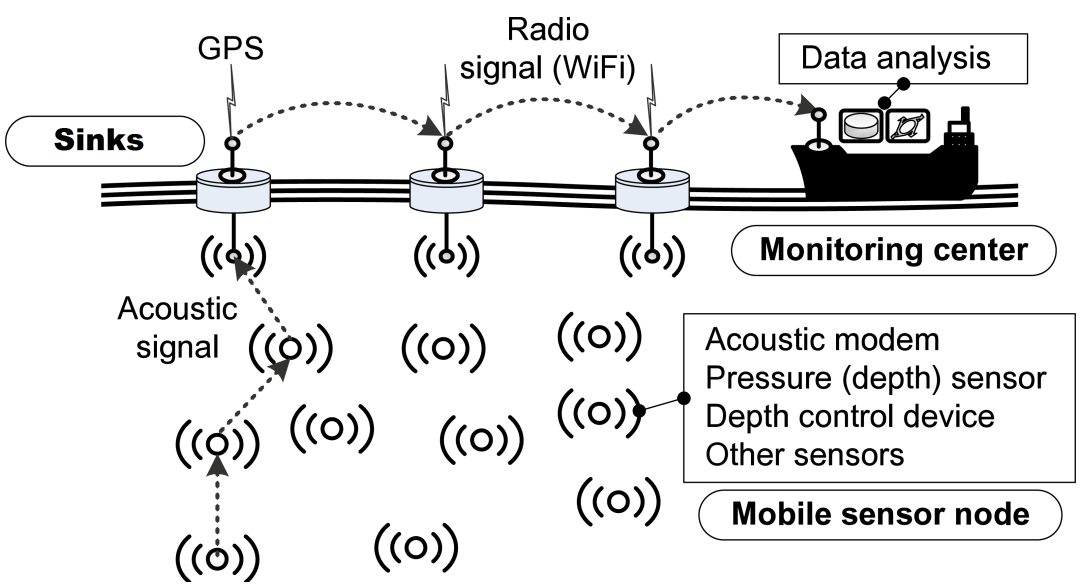

Figure 4.1: Drifting 3D underwater wireless sensor network architecture [113], displaying the location of sinks on the surface.

used to relay the packet constitutes a path. Packets may have to be transmitted over multiple hops across UWSNs, which may be several kilometres wide and several hundreds of metres to a kilometre deep [113], because of the low range of underwater acoustic communications, in the hundreds of metres [118]. The role of routing in such networks is to determine the path to a destination node [11]

Many routing protocols proposed for land-based ad-hoc networks and WSNs are unsuitable for UWSNs. For instance, reactive routing protocols such as Ad hoc On-Demand Distance Vector (AODV) routing [147] establish a route via networkwide flooding of route request packets. This ensures that if a path exists to the destination, it will overhear the packet. The packet then returns along the sequence of nodes it visited to get to the destination, to the source node, which then uses this path to route packets. However, as underwater nodes are mobile due to current advection, the nodes in these paths rapidly move out of communications range of each other. This requires a new route to be discovered, necessitating frequent flooding and making these protocols inefficient. Geographic routing protocols [114] that forward packets to nodes based on how close they are (in terms of their physical distance) to the destination are complicated by the difficulty of full-dimensional localization underwater. GPS can only be used on the sea surface, due to the rapid attenuation of RF waves underwater [190]. Nodes must trilaterate their position via the time of arrival of acoustic signals, which requires the node to be within indirect range of 3 nodes on the surface which are able to determine their location. This condition cannot always be guaranteed. Also, this is energy-intensive (communications-wise) to maintain.

This has resulted in a number of routing protocols being developed over the past 
few years specifically for UWSNs $[112,113,134,148,169,200,205]$ that attempt to address the challenges presented by mobility and the harsh underwater channel. For qualitative surveys of UWSN routing protocols, the reader is referred to $[19,53]$. One category of underwater routing protocols that has emerged is pressure routing protocols $[113,134,205]$.

Pressure routing protocols are a subclass of geographic routing protocols. They circumvent the problem of full-dimensional localization being often unobtainable underwater, by requiring of nodes' location information, only their depths. A node can easily individually obtain its depth by being equipped with an inexpensive strain gauge-based pressure sensor [100]. The measured pressure can then be converted to depth using appropriate formulae, where pressure varies reliably as a function of depth in the ocean and the accuracy of such formulae is around $0.1 \%$ [63].

Data packets from sensors are transmitted via multi-hop communication to sinks in UWSNs. In pressure routing protocols, packets are forwarded by nodes higher up in depth than the sending node. The rationale for this is that as sinks are located on the water's surface, it is highly likely a packet will be received by a sink by forwarding packets upwards. Sinks deliver data to end-users on the surface, often on land, which may be up to an ocean's width away. Hence, sinks need to communicate with the surface station via long-radio radio or satellite links, which requires them to be above water [11]. A packet is considered to be successfully delivered to the monitoring station if arrives at any sink, as each sink has the longrange communication capabilities to transmit the data to the centre [205]. Pressure routing assumes that sinks are sufficiently distributed on the surface so that the packet is received by at least one sink.

Nodes within broadcast range of the sender can decide whether to forward on a packet based on its depth, and the sender's depth embedded in the packet header. The sender can also query the depths of its neighbours to select which node should forward its packet. The advantage of pressure routing protocol is its efficiency in communications terms [113], avoiding the need for network-wide flooding of control messages to establish paths as in proactive or reactive routing protocols. In either case, a forwarding decision is made by an exchange of neighbour information only.

In this study, the performances of two pressure routing protocols are evaluated: DBR [205] and Hydrocast [113]. 


\subsubsection{Depth-Based Routing (DBR)}

The first pressure routing protocol to be proposed was Depth-Based Routing (DBR) [205]. Progress is made by packets towards the surface by only being forwarded by nodes that are higher by $d_{\text {th }} \leq 0$ or more than the sender, as depicted in Figure 4.2 .

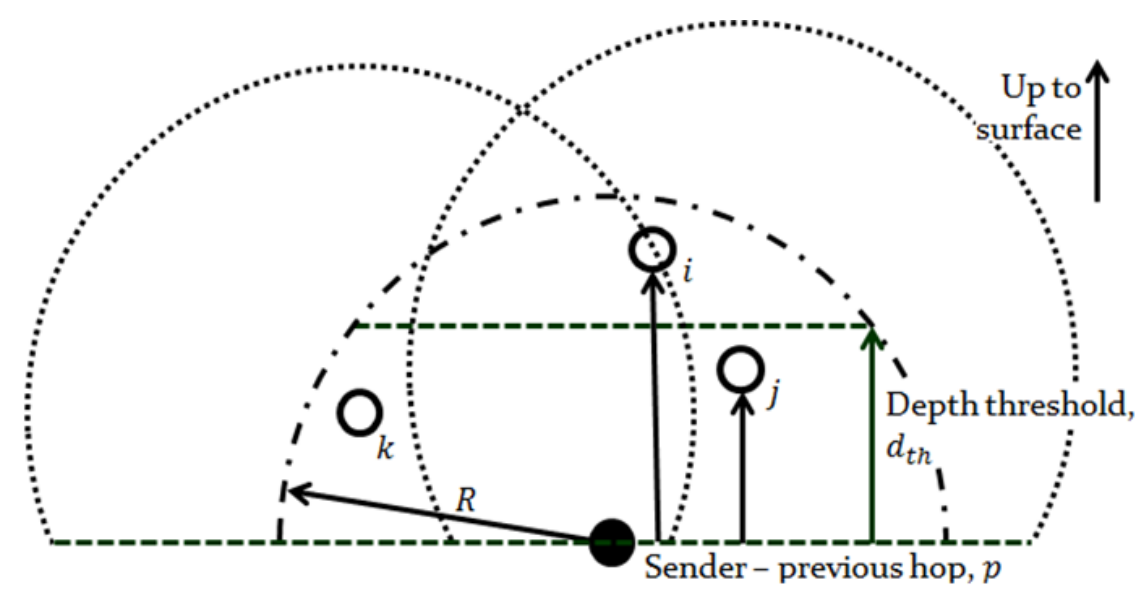

Figure 4.2: Forwarding node selection in pressure routing. The dotted circles indicate the communications / interference ranges of nodes $p, j$ and $k$, approximated by a binary disk model.

The data packet broadcast by the sender includes its depth in the header. Nodes that overheard the transmission decide to forward it if the difference between this value and the sensor's own measured depth exceeds the depth threshold, $d_{\mathrm{th}}$. A problem is that there may be several eligible neighbours. If they all forwarded the packet, it would create redundant transmissions and collisions. To avoid this, a holding time parameter is defined, $\delta$. Nodes wait a holding time that varies linearly with the depth difference:

$$
\left.T_{\mathrm{H}}=\alpha\left(R-\left(d_{\mathrm{p}}-d_{\mathrm{c}}\right)\right)\right), d_{\mathrm{th}} \leq d_{\mathrm{p}}-d_{\mathrm{c}}
$$

before forwarding the packet, where $d_{\mathrm{c}}$ is the depth of the current node and $d_{\mathrm{p}}$ is the depth of the packet's previous hop. The coefficient $\alpha=\frac{2 R}{v_{0}} \delta$, where $R$ is the maximal transmission range of the sensor and $v_{0}$ is the speed of sound underwater. Neighbours that overhear the packet before their holding time has elapsed cancel their transmission of the packet.

The holding time definition favours forwarders that are higher up, causing higher nodes closer to the sinks to forward the packet first. Lower waiting nodes that hear the following broadcast are able to cancel their now redundant transmission. If queueing and processing delays and transmission duration are negligible, then the 
packet first broadcast by node $i$ will be received in time by lower node $j$ to cancel its own transmission if $d_{j}-d_{i} \geq \delta$ [205]. A smaller value of $\delta$ can prevent more redundant broadcasts but at the expense of longer holding times. When the packet transmission delay is included, the broadcast is received in its entirety in time to prevent the lower node from sending if

$$
\alpha>\frac{t_{\mathrm{pi}}-t_{\mathrm{pj}}+t_{\mathrm{ij}}+t_{\mathrm{data}}}{d_{\mathrm{j}}-d_{\mathrm{i}}}
$$

where $t_{\mathrm{ab}}$ is the propagation delay from node $a$ to node $b$ and $t_{\mathrm{data}}$ is the transmission delay of the data packet.

\subsubsection{HydroCast}

Proposed later, HydroCast [113] avoids a number of issues that occur in DBR: redundant packet transmissions from the hidden terminal problem, long holding time delays and void areas.

The holding time in DBR does not completely prevent redundant transmissions, which results in congestion and extra energy expended in communications. A neighbour may not know to cancel its transmission because of the hidden terminal problem, where the node is in communications range of the sender to consider forwarding the packet, but is not in range of another higher neighbour that forwards the packet first, but this transmission cannot be detected and acted upon. This is illustrated in Figure 4.2 with nodes $k$ and $j$.

In HydroCast, beacons are periodically broadcast by nodes to their neighbours within a two-hop radius. Each beacon contains the node's depth and time of transmission, so based on the time of arrival the receiving node can determine its distance to the sensor. With the pairwise distance of its neighbour's neighbours known, a set of hidden terminal-free neighbours can be found by each node. We note that in practice the distances may have some error because of a lack of synchronization between the clock in the broadcasting node, labelling the transmit time, and the clock in the receiving node, recording the arrival time. Time synchronization is difficult to achieve underwater because of a lack of access to the Global Positioning System (GPS) [190] and nodes being unable to communicate their clock states directly due to the speed of underwater propagation being non-negligible [117]. Prior to transmitting, a heuristic algorithm is used to find a cluster of hidden terminal-free nodes $\Gamma$ lying above the sender that, out of the set of all its neighbours, would maximize the expected depth-wise advance to the surface if a packet was forwarded 
to them. The ID numbers of the nodes in this set are included in the packet header, and only those nodes in the forwarding set will attempt to forward the packet.

Like DBR, forwarders wait a linear holding time given by (4.1) before transmitting. In DBR, which uses a fixed value of $\alpha$, forwarders may have to wait an excessive amount of time even if there are no neighbours that would transmit first. With the forwarding set, the sender knows what are the potential forwarders. The sender can then set an optimal value for $\alpha$ that suppresses all redundant transmissions, while leading to the minimum holding time for doing so. Recall that the holding time parameter is able to cause the transmission from node $i$ to suppress a redundant broadcast from a lower sensor $j$ if it is given by (4.2). Then a dynamic value for the parameter that suppresses all transmissions can be found as

$$
\gamma=\max _{i, j \in \Gamma}\left(\frac{t_{\mathrm{pi}}-t_{\mathrm{pj}}+t_{\mathrm{ij}}+t_{\mathrm{data}}}{d_{\mathrm{j}}-d_{\mathrm{i}}}\right)
$$

This holding time parameter is also included in the header, and used by the nodes in the forwarding set for this transmission.

In greedy forwarding, a problem may arise when the node is in a void area where there are no neighbors that move the packet closer to the destination. In DBR, the protocol reaches a dead-end. However, HydroCast specifies a local lower-depth-first recovery mode where nodes that are local maxima are required to maintain a route along the floor of the void to another node of lesser depth. This enables packets to be routed out of the void area and greedy forwarding can be resumed at this point. Both local maxima and surface status can be obtained from beacon neighbour status. Details of the recovery mechanism are not available in [113].

\subsection{Related work}

Pressure routing protocols represent an innovative adaptation of geographic routing to the unique underwater environment. It is unclear what their performance in actual deployment will be, as they have only been validated in simplistic current mobility models. Past performance studies of DBR and HydroCast are surveyed in this section, and deficiencies in the realism of the mobility models employed are highlighted.

DBR was evaluated in simulation alongside Vector Based Forwarding [204] in [205]. Between 100-600 nodes, with a transmission range of $100 \mathrm{~m}$ were randomly deployed in a $500 \mathrm{~m} \times 500 \mathrm{~m} \times 500 \mathrm{~m}$ box. A single source node at the bottom of the box generated data for transmission at the rate of one packet per second, and the 
performance of the protocol for delivering this data to sinks was measured for various values of the protocol parameters: $\alpha$ and $d_{\mathrm{th}}$. Nodes moved according to an unrealistic random walk mobility model for different speed settings. The motion of nodes in this scheme was uncorrelated, whereas in real-life sensors are advected by underlying current structures such as eddies and tides, and the movement of devices that are closely spaced should be similar [51]. Nodes remained within the initial box where they were deployed. Single values of the results, such as the packet delivery ratio, representing the average over an unspecified time, were only reported.

HydroCast and DBR were simulated and their performance compared in [113]. The nodes were advected by a current described by a simplistic depth-invariant 3D extension of the MCM model [32]. Reasons why this model is likely to be inaccurate and the current represented by MCM is depth-decaying were presented in Section 3.4. A network, ranging in size from 100-450 nodes, was initially deployed in a $1000 \mathrm{~m} \times 1000 \mathrm{~m} \times 1000 \mathrm{~m}$ box. The transmission range of each sensor was $250 \mathrm{~m}$. Each node transmitted a packet containing its sensed information every 60 seconds. The network was left to drift for 1 hour and the value of the packet delivery ratio and other metrics, averaged over each node and the entire duration to give a single value for the run, presente.d c

\subsection{Simulation settings}

A simulation scenario was developed to investigate the impact that realistic current mobility has on the connectivity of 3D drifting UWSNs and these routing protocols. The simulations were run on QualNet 5.0. The network setup, such as the network size, energy consumption and physical layer used, is based on the original DBR validation simulation [205] with parts of the HydroCast simulation in [113], so that valid comparisons with these papers' results can be made. Now though, instead of a stochastic or depth-invariant current affecting the region, the flow is described by Current 1.

\subsubsection{Simulation scenario}

As for the DBR validation scenario [205], nodes are randomly deployed in a $500 \mathrm{~m}$ $\times 500 \mathrm{~m} \times 500 \mathrm{~m} 3 \mathrm{D}$ space with an approximate communications range of $100 \mathrm{~m}$. In that scenario, network traffic is provided by a source at the bottom. We retain this concept, as such monitoring may appear in oil plume tracking applications. As 
described in Section 2.4, after the Deepwater Horizon spill a $2 \mathrm{~km}$ wide body of oil was found between 1000-1200 m depth and drifting with the surrounding water. This scenario envisions an oil plume occurring along the bottom layer of the box. The network is deployed to report back in-situ measurements made by source nodes located at depth $500 \mathrm{~m}$ along the plume. The plume is an extended feature, so rather than one source node as in [205], 10 sources are randomly placed within the initial deployment area at $500 \mathrm{~m}$ depth. Ten sinks are randomly placed on the water's surface, with between $n=100-600$ other sensors making up the rest of the network.

Sources transmit on average one 50 byte packet per second, before other headers are added, at $50 \mathrm{kbps}$ [113]. The original DBR scenario data rate of $10 \mathrm{kbps}$ led to too much congestion, where network capacity was having the most effect on the performance instead of the routing protocols. The size of the data packet with all headers included except the routing header is 94 bytes, as QualNet requires adding UDP and IP headers. The UDP and IP headers are there to satisfy some unmodifiable internal code checks made by the closed-source QualNet simulator before it will send out a packet. The UDP and IP protocols do not actually run during the simulation, and the only impact these headers have is to pad out the packet size. The packet size is consistent (of the order of) with previous performance studies of the pressure routing protocols, with Yan and Shi [205] utilizing 50 byte packets, allowing our results to be compared. Packets are generated at the source every second but are not transmitted until after a short delay, uniformly randomly selected between 0 and $1 \mathrm{~s}$, to prevent synchronization.

\subsubsection{Physical layer}

In previous simulations evaluating these pressure routing protocols, a transmission range of $100 \mathrm{~m}$ was used with a node receiving any packet transmitted from within this range perfectly in the absence of collisions and not receiving the packet correctly if any collision occurred [205]. This transmission range is consistent with commercial underwater acoustic modems, such as the LinkQuest UWM1000 [118]. In this work, we define the physical layer in our simulation to reproduce this behaviour so that our results can be compared. The path loss is defined by the Urick propagation model [197], $A(d, f)=d^{k} a(f)^{d}$, where $d$ is the distance, $f$ is the signal frequency, the spreading factor $k=1.5$ and Thorp's formula is substituted for the absorption coefficient $a(f)$. The underwater propagation speed is fixed at $1500 \mathrm{~ms}^{-1}$. Rayleigh fading is assumed and the bit error rate (BER) at the receiver is calculated for BPSK (binary phase shift keying) modulation at that signal-to-noise ratio (SNR) [113]. A 
packet is only successfully received if there are no bit errors. The transmission power is set to $105 \mathrm{~dB}$ re $\mu \mathrm{Pa}$ and the receiver sensitivity and noise power to $75 \mathrm{~dB}$ so that with path loss the SNR of the signal is $0 \mathrm{~dB}$ and a packet cannot be received at a distance of $100 \mathrm{~m}$.

\subsubsection{DBR}

For determining $\alpha$ in DBR, the simulation physical layer is employed. This gives the maximal transmission range $R$ as $100 \mathrm{~m}$ and the speed of sound underwater $v_{0}$ as $1500 \mathrm{~ms}^{-1}$. The DBR data packet header is 16 bytes long in this simulation and the total size of the data packet at the physical layer is 110 bytes.

\subsubsection{HydroCast}

HydroCast is configured with the same parameters as those reported in [113]. Beacons are exchanged every 30 seconds plus a jitter that is interpreted here as an additional delay uniformly randomly selected between $0-30$ seconds. The beacon data is set to expire after 90 seconds if not updated by the same neighbour. Parameter $k$ (the number of random vectors checked for domination to determine whether the node is on the network face) is set to 20 and a Bloom filter of size 150 bits is used to store the forwarding set. The variable $\beta=0.5$ and the maximum allowable delay per hop is $\gamma=1.0 \mathrm{~s} / \mathrm{m}$.

HydroCast is evaluated with and without the recovery mechanism in this paper, labelled as HydroCast-RECOVERY and HydroCast-GREEDY respectively. With lower-depth-first recovery, packets that end up in a local maximum are flooded by nodes on the void floor surface until a higher sensor is reached and switches. The protocol then switches back to greedy forwarding. Nodes that receive a flooded packet do not wait a holding time as the forwarding set is not known in order to adaptively set $\alpha$. The sizes of the flooding and data packets at the physical layer in QualNet are 126 bytes and 161 bytes. The beacon size varies with the number of neighbors. The maximum size observed was 204 bytes.

\subsubsection{Mobility}

All nodes in the simulation move under Current 1, defined in Section 3.7. As a coastal current model, this choice is also appropriate for this oil plume monitoring scenario, since many of the offshore oil platforms in the US are located off the coasts of Texas 
and Louisiana [9]. The model accounts for the measured decline in the speed of the current on which the model is based, by adjusting the velocity scaling of the $2 \mathrm{D}$ flow structure at each layer to match the experimentally obtained values. The parameters from Table 3.5 are used for the current, where $\tau=0.03$ days, $\xi=1 \mathrm{~km}, A^{\prime}=1.2$, $c_{x}^{\prime}=0.12, k^{\prime}=2 \pi / 7.5, \omega^{\prime}=0.4$ and $\epsilon^{\prime}=0.3$, to give the appearance of a coastal current. The normalization factor is specified by Figure 3.15. The box in which the network is initially deployed is displayed in Figure 4.3, where the streamlines also highlight the difference in the current between depths.

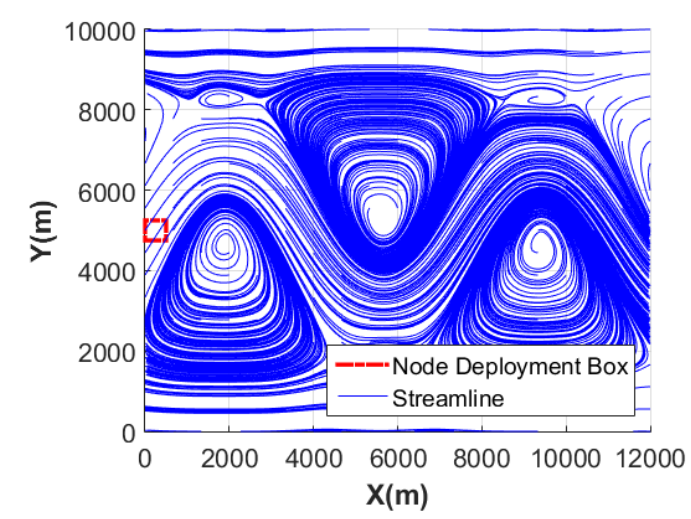

(a)

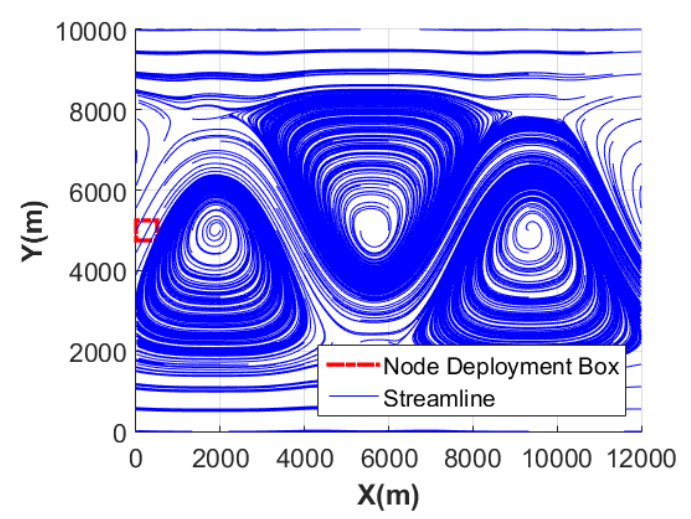

(b)

Figure 4.3: Current 1 mobility model streamlines in the comoving frame of reference at $t=0 \mathrm{~s}$ at (a)depth $=0 \mathrm{~m}, s_{c}=0.39 \mathrm{~ms}^{-1}$ and (b)depth $=500 \mathrm{~m}, s_{c}=0.22 \mathrm{~ms}^{-1}$. Model parameters after a typical coastal current: $A=1.2, c=0.12, k=2 \pi / 7.5, \omega=0.4, \epsilon=0.3$, where non-dimensional units are $1 \mathrm{~km}$ for space and 0.03 days for time.

Since the simulation of the network in realistic current mobility is the main contribution of this chapter, a more detailed explanation of how the Current 1 model is implemented in the simulator is provided. QualNet comes with implementations of random waypoint and pedestrian mobility models. Both models are called by each node at the start of the simulation, which then immediately generate all the waypoints for the node to follow for the full simulation duration. For specifying other forms of mobility, QualNet provides the ability to supply it with a waypoint file consisting of a sequence of position co-ordinates for each node, and times for the node to reach each point [166]. This file is used to initialize the simulation and defines the motion of the network for the rest of the simulation. During the simulation, nodes will move so that at the times given in the file, the sensor will be positioned at the associated co-ordinates. In between these times, the co-ordinates of a node is given by the linearly interpolated position between waypoints. No template for dynamic 
mobility exists. All mobility in QualNet is pre-generated for the entire simulation duration and does not change in response to events.

QualNet's file-based mobility model was utilized to specify the movement of nodes in the scenario. The position of nodes every $10 \mathrm{~s}$ was found by numerically integrating the velocity of each node, defined by (3.15), in MATLAB employing a fourth-order Runge-Kutta method [37]. This was carried out using MATLAB's ode45 [126] function. The co-ordinates were written to a file that was then fed into QualNet.

The function ode45 has prototype

$$
[\mathrm{T}, \mathrm{Y}, \mathrm{TE}, \mathrm{YE}, \mathrm{IE}]=\text { ode45 (odefun, tspan, } \mathrm{x} 0 \text {, options) }
$$

Given a velocity function

$$
\frac{d \mathbf{x}}{d t}=\mathbf{v}(t, \mathbf{x})
$$

ode45 solves the system of differential equations for $\mathbf{x}$ (the future node co-ordinates needed to specify the waypoint file) at times given by $\operatorname{tspan}=\left[t_{0}, t_{1}, t_{2}, \ldots, t_{\mathrm{f}}\right]$ and initial conditions $\mathbf{x}\left(t_{0}\right)=\mathbf{x} 0$, where odef un is a function handle that evaluates $\mathbf{v}(t, \mathbf{x})$. In our usage, the time span between the start and end of the simulation, divided up into $10 \mathrm{~s}$ intervals, is entered as tspan.

The function ode45 implements a fourth-order Runge-Kutta method for numerically integrating the equation. In numerical techniques, $\mathbf{x}\left(t_{1}\right)$ is estimated by dividing the interval $\left[t_{0}, t_{1}\right]$ into sample times or mesh points, $t_{0}<t_{\mathrm{a}}<t_{\mathrm{b}}<\ldots<t_{1}$. Starting from $t_{\mathrm{a}}$ an approximate value for $\mathbf{x}$ at this point, $\widetilde{\mathbf{x}}_{\mathrm{a}}$, is calculated as a function, depending on the method, of the estimated quantities of $\mathrm{x}$ at the preceding sample times. The formula is then used to find $\widetilde{\mathbf{x}}$ for successive time points. The interval between sample times is referred to as the step size.

The actual values for $\mathrm{x}$ are then considered to be given by $\widetilde{\mathbf{x}}$, where the accuracy of the approximation depends on the order of the method employed and the step size. For a fourth-order method, the error between the approximation and exact solution value is bounded by a multiple of $h^{4}$ where $h$ is the step size. For most practical purposes, the fourth-order Runge-Kutta method is indistinguishable from the actual solution [139]. Further explanation of how the Taylor series expansion of the velocity function is used to generate the method, and its accuracy can be found in [139]. The solver error tolerances used in this simulation are left unchanged from their default settings of AbsTol $=1 \times 10^{-6}$ and RelTol $=1 \times 10^{-3}$. 


\subsubsection{Metrics}

The performance of the routing protocols is evaluated by the achieved packet delivery ratio (PDR), the end-to-end delay and the energy consumption. The PDR of a source is the proportion of packets received correctly by at least one sink over the total number of packets sent. The time between when a packet is sent until the earliest time it reaches a sink comprises the end-to-end delay, and only considers delivered packets. The total energy consumption of the network is used to judge the overhead of the protocol.

The values of these metrics are assessed throughout a 3 hour period postdeployment, in order to understand the impact current mobility has on UWSN routing. Two types of simulations were run. In the first case the mobility model was processed offline to calculate the distribution of nodes at half-hourly intervals $t=\{0.0,0.5,1.0,1.5,2.0,2.5,3.0\} \mathrm{h}$ and their motion $200 \mathrm{~s}$ either side of this time. The simulation was then executed. The network was left to run for the first $200 \mathrm{~s}$, with statistics only being gathered during the latter $200 \mathrm{~s}$ once the network has stabilized. This provides a discrete snapshot of the PDR and end-to-end delay at the corresponding point in the network's drift. The scenario was run over the full 3 hour duration in the second type to record the overall energy consumption.

We also consider what impact mobility has on the network topology, independent of any protocol that may be running on the network. The node connectivity at a time instant $t$ is evaluated. A node is connected if a feasible path exists to a sink, where the degree of connectivity is equal to the number of distinct sinks the node is able to reach. This is measured by examining the node positions at $t$. An induced communication graph [65] is generated from these positions, where the vectors are the set of nodes and an edge exists between any two nodes if the distance between them is less than $100 \mathrm{~m}$, the maximal transmission range. A brute-force search along the graph is used to determine how many sinks are connected via paths to a source node, giving the source's degree of connectivity. The connectivity provides an indication of the best-case deliverability of packets from a node, should an ideal routing protocol be able to find and disseminate these paths and congestion along these paths be eliminated.

Each simulation is repeated 20 times with different seeds used to generate the initial $t=0$ s randomly deployed node distributions. The median value of 20 runs are reported with uncertainty bars consisting of the $25 \%$ and $75 \%$ quartiles of measured results. 


\subsection{Performance results}

In this section, we discuss the performance based on our four metrics: degree of connectivity, PDR, end-to-end delay and energy consumption.

\section{Degree of connectivity}

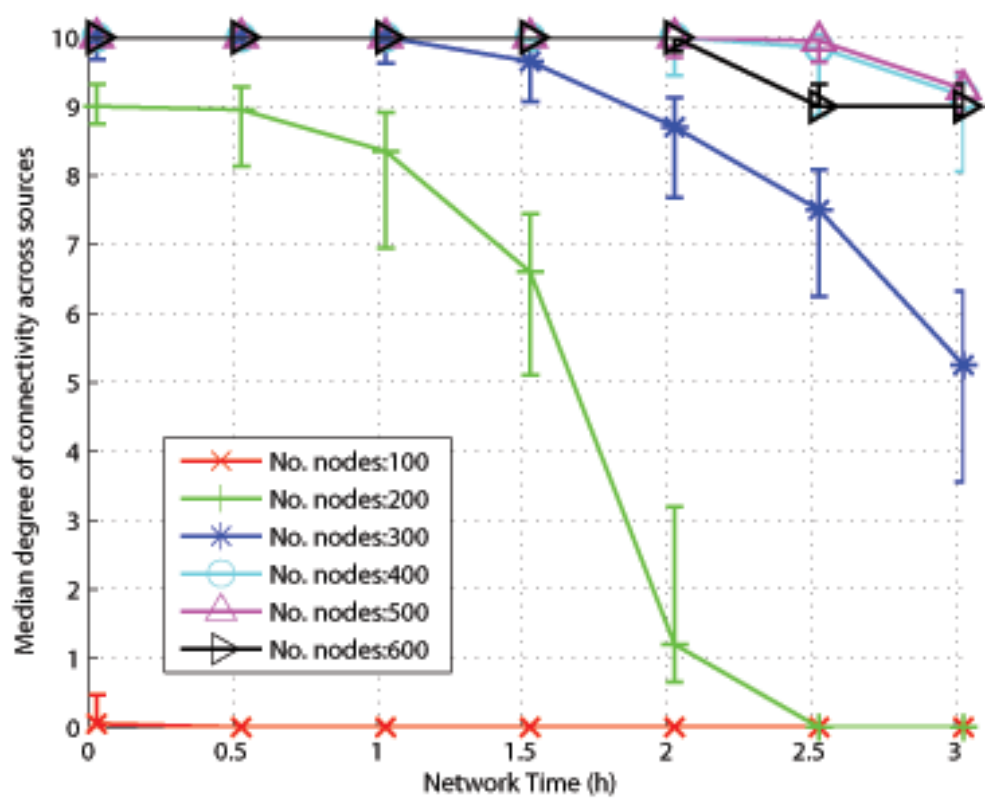

Figure 4.4: Median degree of ideal connectivity across sources under different network sizes, against time.

Figure 4.4 displays the connectivity results and shows that sources lose contact through to the sinks on the surface with time, which is expected. The speed inside the jet decreases with depth, with the speed inside the jet centre being $0.39 \mathrm{~ms}^{-1}$ on the surface, falling to $0.22 \mathrm{~ms}^{-1}$ at $500 \mathrm{~m}$ depth. This causes the various horizontal layers of nodes in the network to shear, as visualized in Figure 4.5, with nodes lower down moving slower eastward and appearing to lag behind the sensors on the surface. Over time, the separation between nodes at different layers cause more devices to be out of communications range of nodes higher than them. This severs any possible paths to the surface sinks. 


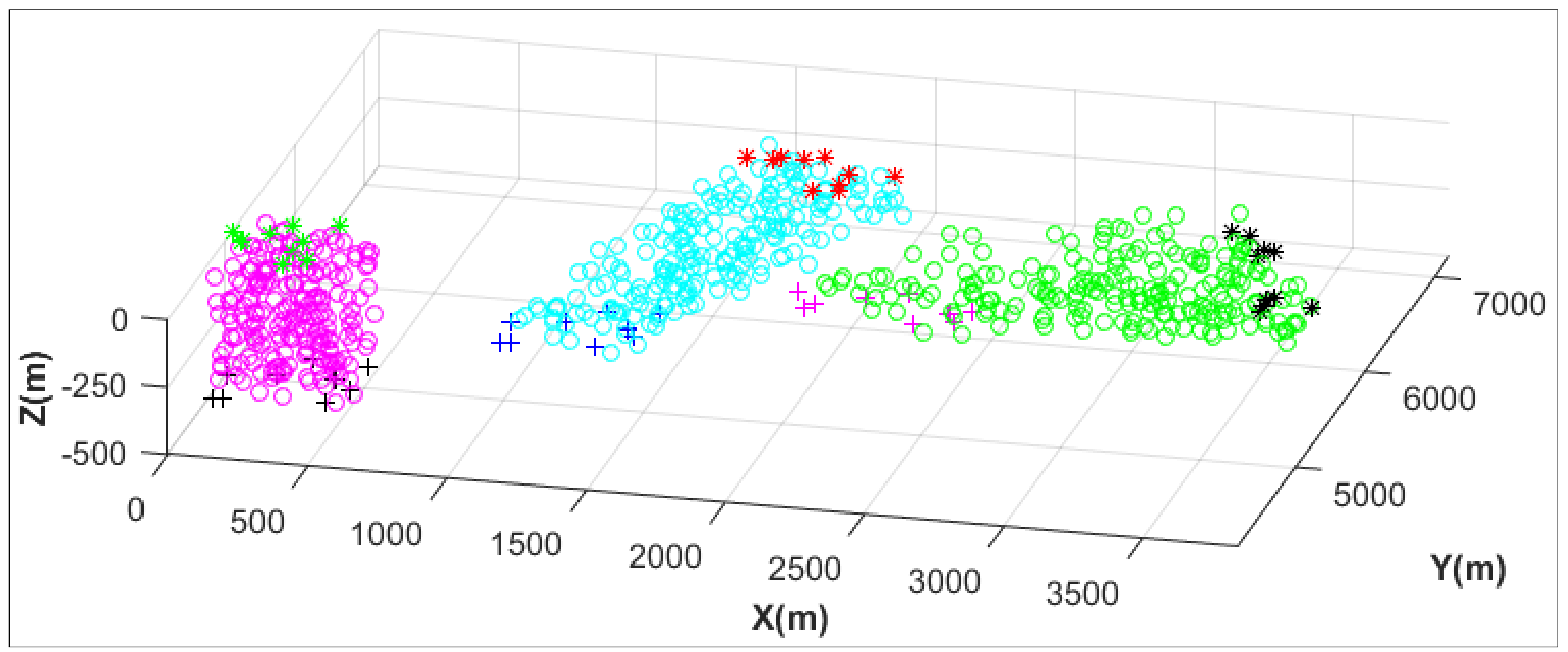

Figure 4.5: Typical positions of nodes, taken from a run, under extended 3D MCM mobility over time. The cross markers denote the positions of the sources, at $500 \mathrm{~m}$ depth, and the asterisks the sinks, on the surface. Initial topology: 200 sensors uniformly randomly deployed in a $500 \mathrm{~m} \times 500 \mathrm{~m} \times 500 \mathrm{~m}$ box. 
Not only that but, as shown in Figure 4.3, when this speed is lower the region of recirculating vortices extends deeper towards the center of the meandering jet. At the surface the initial deployment area is squarely in the meandering jet. However, at $500 \mathrm{~m}$ depth, the bottom right corner is grazing the edge of a vortex, where it is detrained from the jet and not picked up until the next crest passes over it, which ultimately causes nodes located at this depth to not progress downstream while waiting for the meander crest or trough to wash over them.

After $t=1.5 \mathrm{~h}$, sources are completely disconnected in the 100-node network and this network size is omitted from later figures as the scenario does not produce any meaningful results. The 200- and 300-node topologies display a large decrease in the degree of connectivity between $t=0-3 \mathrm{~h}$. For $n=400-600$, sources are still connected to a median of at least 8 out of a possible 10 sink nodes after $t=3 \mathrm{~h}$.

\section{PDR}

The PDR performance of DBR and HydroCast are plotted separately in Figures 4.6 and 4.7. The parameters used for DBR are $d_{\mathrm{th}}=0 \mathrm{~m}$ and $\delta=100 \mathrm{~m}$, the maximal transmission range, which is found in [205] to give the best end-to-end delay with negligible increase in energy consumption.

In the cases of both DBR and HydroCast-GREEDY, the PDR has declined to zero or near-zero within time $t=3 \mathrm{~h}$ of the network being deployed. The PDR increases with the number of nodes deployed, although the benefit becomes minor above 400 nodes where boosting the network size to 600 nodes, a $33 \%$ increase, only changes the initial PDR of DBR by $5 \%$. However, even with 600 nodes, the PDR of the network when DBR is used at $t=3 \mathrm{~h}$ is $3.3 \%$ compared to $87 \%$ at $t=0 \mathrm{~h}$. Nodes do not fail or run out of energy in the scenario, so this dropoff is entirely due to mobility.

The performance over time of HydroCast-RECOVERY is completely different. An 18\% dropoff in PDR between $t=0 \mathrm{~h}$ and $3 \mathrm{~h}$ is measured for the network sizes $n=400$ and 600 nodes that is consistent with the change in connectivity degree observed in Figure 4.4, which decreases from 10 to more than 8 sink nodes. This indicates that sufficient routes to the surface still exist that can be followed by downward flooding around void areas, but which DBR and HydroCast are prevented from exploiting if only greedy forwarding to upper nodes is allowed.

The PDR of HydroCast-GREEDY is lower than DBR and follows a similar falloff trend with time. On average, the PDR is $53 \%$ less at $t=0 \mathrm{~h}$. The PDR of HydroCast is also worse than DBR in [113], but, in their results, enabling lower- 


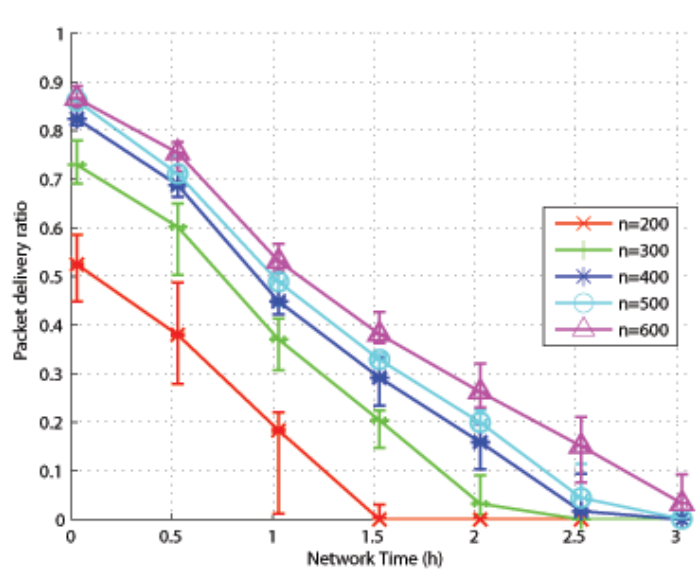

Figure 4.6: Median packet delivery ratio of DBR under different network sizes; $\delta=100 \mathrm{~m}$, $d_{t h}=0 \mathrm{~m}$.

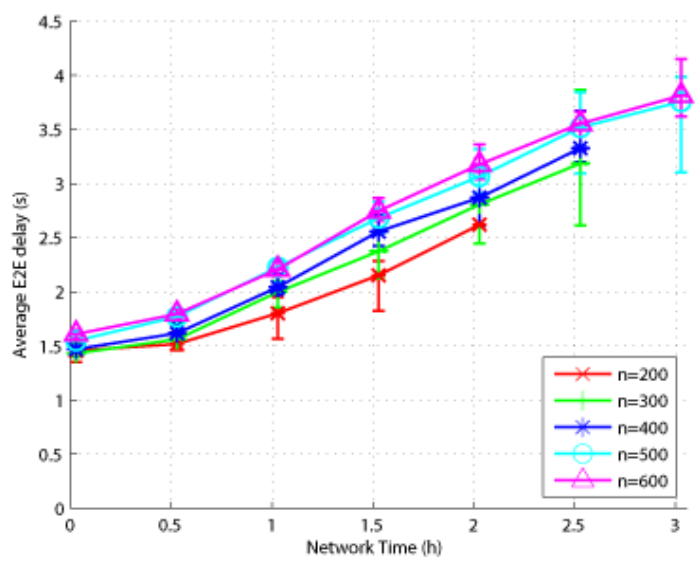

Figure 4.8: Median end-to-end delay of DBR under different network sizes; $\delta=100 \mathrm{~m}, d_{t h}=$ $0 \mathrm{~m}$.

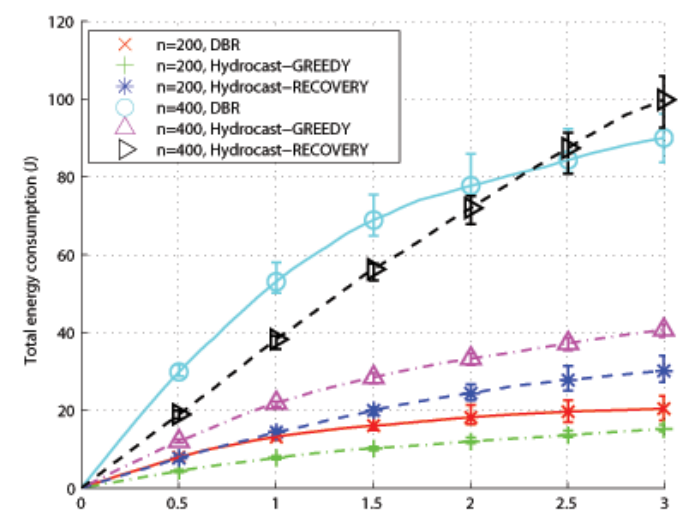

Figure 4.10: Median total network energy consumption for DBR and HydroCast, where the number of nodes $n=200$ and 400. DBR: $\delta=100 \mathrm{~m}, d_{t h}=0 \mathrm{~m}$.

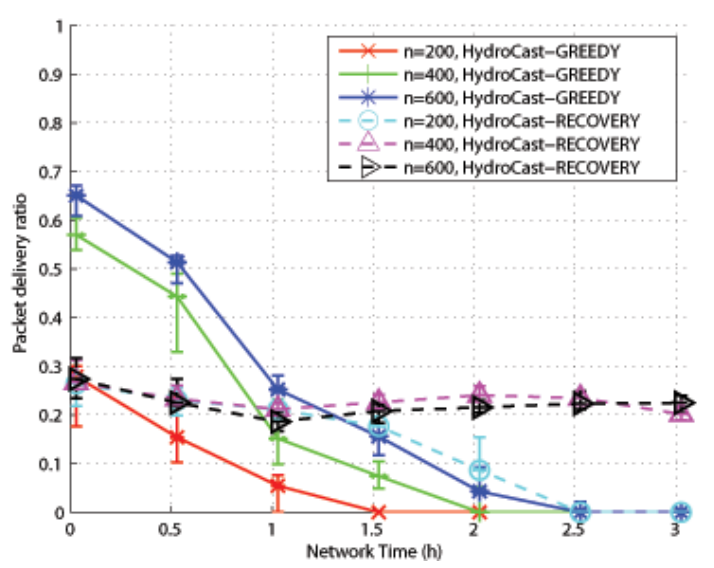

Figure 4.7: Median packet delivery ratio of HydroCast with lower-depth-first recovery flooding disabled and enabled under different network sizes.

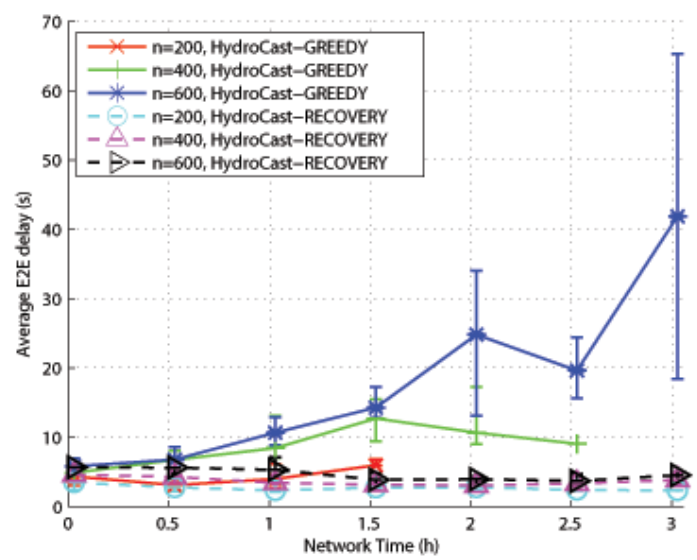

Figure 4.9: Median end-to-end delay of HydroCast with lower-depth-first recovery flooding disabled and enabled under different network sizes.

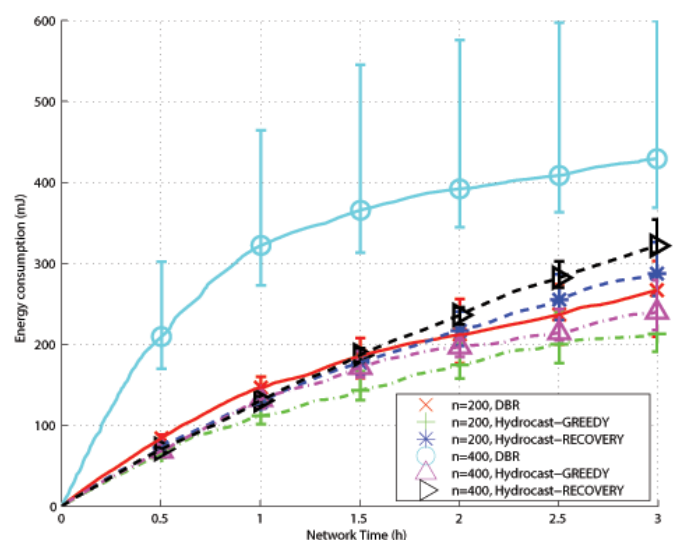

Figure 4.11: Maximum individual node energy consumption for DBR and HydroCast, evaluated out of all sensors in the network, where the number of nodes $n=200$ and 400. DBR: $\delta=100 \mathrm{~m}, d_{t h}=0 \mathrm{~m}$. 
depth-first recovery leads to HydroCast outperforming DBR. Here, the PDRs of HydroCast-RECOVERY for different network sizes are initially identical to within uncertainties, but have less than half the value of DBR or HydroCast-GREEDY. The face routing protocol used by [113] is not specified. Surface flooding and route discovery packets are both mentioned. It may be that paths are stored in [113], whereas in this implementation the packet is always flooded, and this results in more channel contention and dropped packets. Furthermore, in [113] each node in the network sends packets. The lower PDR in our results is attributed to HydroCast having greater difficulty in routing packets originating from the bottom of the network, compared to the average over nodes at all layers, which must be relayed over more hops to reach its destination.

\section{End-to-end delay}

The end-to-end delay over $200 \mathrm{~s}$ with the network at different time states is plotted for DBR in Figure 4.8. The latency at $t=0 \mathrm{~h}$ is virtually identical for different network sizes, which matches the results in [205]. As the network drifts apart with time, the delay increases. This is attributed to packets having to travel laterally over a greater total distance to sinks that have moved further ahead in the jet. Some lines terminate before $t=3 \mathrm{~h}$ when no packets are received by any sinks to calculate the latency. Figure 4.9 shows the end-to-end delay for HydroCast. The latency for HydroCast-GREEDY is higher than HydroCast-RECOVERY. The large uncertainty bars observed as time increases arise when the existence of routes is highly dependent on the distribution of nodes in a sparse network. The static end-to-end delay over time of HydroCast with lower-depth-first recovery suggests that the increasing lateral separation of sinks and sources under drift is being balanced by nodes having fewer neighbours, which means lower holding times before forwarding.

\section{Energy consumption}

Energy management underwater is crucial. Not only does acoustic communications requires more power than terrestrial RF signals but UASNs are less densely deployed than land-based WSNs, and therefore less tolerant to the loss of links. Two types of energy graphs are plotted using the running results from the full 3 hour network simulations. The total network energy consumption summed across all devices is plotted in Figure 4.10 for $n=200$ and 400 sensors. As expected, the larger and hence denser network has higher absolute energy consumption as there are more 
receivers per hop and redundant forwarders.

The maximum energy consumption by any sensor in the network up to this point in time is graphed in Figure 4.11. The intersection of any curve with the sensor battery capacity allows the lifetime of the network to be determined, which can be defined as the time until the first node runs out of energy [154]. The results show that the additional forwarder suppression of HydroCast-GREEDY produced by a hidden terminal free forwarding set and adaptive holding time reduces the network energy consumption of the protocol below that of DBR, despite extra header and beacon overhead.

\subsection{Summary}

In this chapter, the performance of two pressure routing protocols: DBR and HydroCast in coastal environments was evaluated in simulation. A realistic 3D current mobility model was employed to describe the flow in the region, whereas previously naive mobility models had been utilized only. The protocols' performances were assessed in an oil plume monitoring-based scenario, where data was generated along the bottom layer of the network, corresponding to the location of the plume.

DBR was found to provide substantially higher packet delivery ratio than HydroCast in the initial network deployments. The median packet delivery ratio was consistently $0.2-0.3$ higher. The explanation for this is because DBR explores more pathways, increasing the changes of packet delivery, whereas HydroCast suppresses the use of forwarders affected by the hidden terminal problem. The tradeoff for transmitting data along more pathways is higher energy usage in DBR, which was observed in the energy consumption graphs.

The results show that void recovery in geographic routing protocols allows the protocol to still operate after the network has drifted over a longer period. The packet delivery ratio of HydroCast only decreases by $18 \%$ after 3 hours, whereas DBR, which only uses greedy forwarding, cannot route any packets to sinks. Current drift can have a rapid degrading effect on the connectivity on sparser networks, even if the number of nodes in these UWSNs is sufficient for the network to be almost fully connected when initially deployed. When networks of 100 nodes were deployed in the simulation, a path existed from each sensor to nearly every sink at the start. After 3 hours of drift, no nodes were connected. 


\section{Chapter 5}

\section{A practical coverage extension node movement scheme for float-based UWSNs}

So far in this thesis, the evolution of untethered 3D underwater wireless sensor networks in realistic depth-varying currents has been studied. It was found that when using nodes that drifted purely with the currents, having no active mobility of their own, the network rapidly dispersed and became disconnected. The ability to perform basic network operations such as routing quickly became disrupted, in a matter of hours, long before other factors hampering node performance such as battery life would become a problem. The conclusion was that current drift was the major impediment to implementing untethered UWSNs.

A method for addressing this problem could be to equip each sensor node with its own propulsion, and utilize a node movement scheme to coordinate the movement of these mobile nodes so that the network remains connected and covering the RoI in spite of the currents.

Underwater mobility can take various forms, as depicted in Figure 5.1. Autonomous underwater vehicles (AUVs) are equipped with multiple propellers for holonomic motion, and can move at will (subject to battery life) to close coverage holes opened up by current drift. The cost of these vehicles is very high, however, with an oceangoing AUV being priced at US\$50,000 - 250, 000 [143]. In contrast, it is relatively simple to travel up and down in water by varying a device's bouyancy. This type of mobility is employed by profiling floats. Buoyancy is typically adjusted in floats by extending or retracting a piston [24,127] or inflating or deflating a bladder [47] Less complex mechanically than AUVs, floats are correspondingly much 
cheaper. Comparing ocean-going types again, the unit cost of an autonomous float is around US\$9000 [178]. Although only vertically actuated, floats can utilize this to change their horizontal velocity due to the variation of currents with depth. By ascending or descending, the device can choose to drift at different speeds and directions given by the current velocity at that depth towards their desired location.

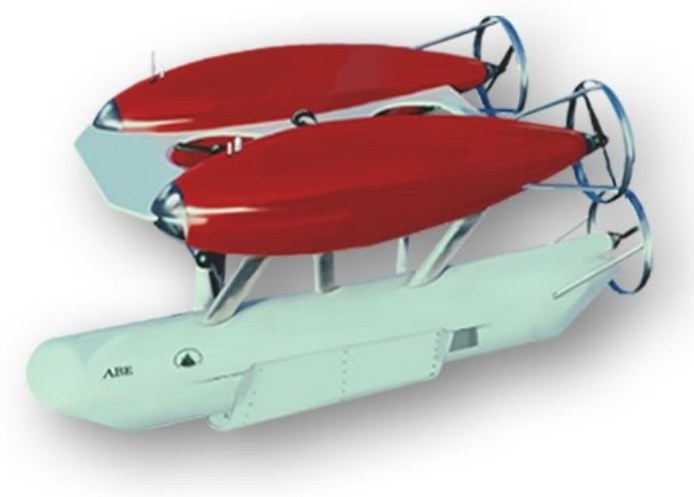

(a)

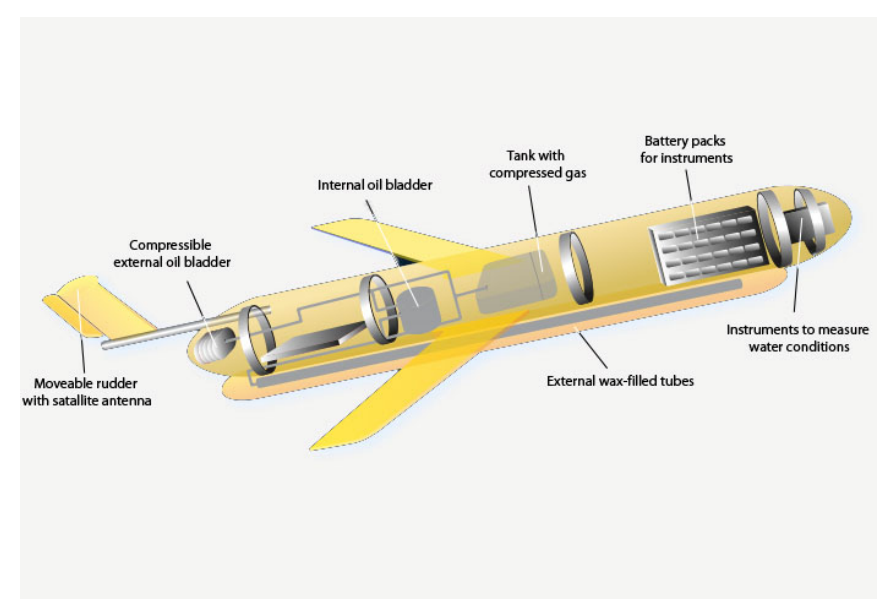

(c)

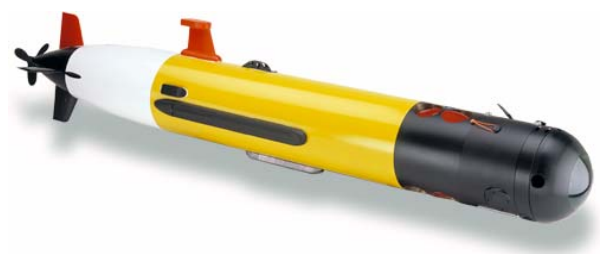

(b)

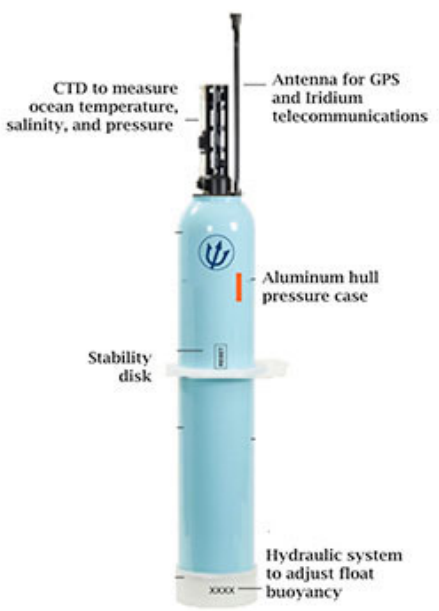

(d)

Figure 5.1: Different classes of untethered unmanned underwater vehicles (UUVs). a) a holonomic multi-thruster AUV, ABE (Autonomous Benthic Explorer) b) a torpedo style AUV with a propeller at its stern and that can steer using its fins, c) a glider and d) a profiling float, which is only directly able to control its depth.

In this chapter, we investigate the use of underwater wireless sensor networks consisting mainly of profiling floats, where the mobility of sensors is co-ordinated by a node movement scheme so that the UWSN maintains coverage of the 3D RoI. Deploying an UWSN of profiling floats instead of AUVs is attractive for a number of 
reasons. With the current level of technology, many more (by an order of magnitude more) nodes could be employed for the same purchasing cost. From a philosophical standpoint, floats fit better with the concept of wireless sensor networks as consisting of cheap, disposable nodes that can be deployed in large numbers [12]. At the same time, by exploiting the currents at different depths floats promise a similar ability to AUVs to be able to move throughout the 3D space. Of course, the reachable positions are dependent on the currents in the region. For instance, if the current everywhere is pointing eastwards no choice of depths will allow the node to end up a westward position, and eventually all profilers will be carried out eastwards of the RoI. It has been shown that, if the currents along the depth profile at each horizontal position positively span the $2 \mathrm{D}$ plane, a float deployed anywhere in the region can reach any 3D location in the region in finite time [181]. In the case of the above scenario, a depth layer where the current is due west will allow the profiler to move to a westward goal location. Many areas in the ocean have been found to have this positively-spanning property [181], providing impetus to our study into float-based UWSNs.

A number of float path planning strategies have already been published. However, these schemes only deal with moving floats to $2 \mathrm{D}$ target locations. These are of limited usefulness to 3D UWSN coverage, where a node needs to arrive at the correct depth as well as horizontal position of a coverage hole. Trajectory planning algorithms to move single floats to 2D target positions are developed by [93,181,182]. 2D formation control of widely-spaced float arrays is investigated in $[42,130]$. Mobility is employed to allow the profilers to disperse to increase coverage and converge for recovery. The time during which the profilers are in transit between commanded depths is ignored in these works, which is equivalent to assuming that they have infinite vertical speed. When real-life vertical actuated speeds were used, the performance of one of these schemes was found to halve [130]. The schemes' floats no longer end up in their expected positions, as the devices experience additional horizontal displacements during their transition between commanded depths.

This chapter presents our novel profiling float node movement scheme for extending the time than an UWSN is able to cover a 3D RoI, hereafter referred to as Scheme 1. A subsequent chapter, Chapter 6, evaluates the performance of our scheme under realistic current mobility. We are the first to address a float movement scheme to cover 3D positions. Scheme 1 does not ignore the non-negligible time spent transitioning between depths by floats. Instead, our solution exploits the horizontal distance moved by floats during this period to more accurately position nodes onto 
coverage holes. A major emphasis in the design of this scheme has been on ensuring its practicality, so that the strategy can be implemented today, as described, with existing technology. Given the low bandwidth and high energy cost of underwater acoustic communications, a mostly communications-less approach is taken. The primitiveness of current predictions made by ocean forecast systems is respected, so floats instead rely on in-situ measured current velocities in the scheme.

This chapter is structured as follows. In Section 5.1, related work on float path planning schemes is surveyed. An important controllability result from the literature is restated in this section. Section 5.2 outlines our distributed node movement scheme for 3D coverage maintenance. Our scheme requires "well-behaved" currents, which floats can exploit to be 3D controllable. In Section 5.3, we find sufficient flow velocity field conditions for 3D controllability. These conditions can be taken to be the type of current that our scheme need to work perfectly in. The two main components of our algorithm, a lightweight trajectory generator, implemented on each individual node, and a one-off network-wide target assignment routine are then detailed in their own separate sections: Sections 5.4 and 5.5 respectively. Finally, Section 5.6 examines the scheme's limitations and Section 5.7 summarizes the chapter.

\section{$5.1 \quad$ Related work}

Floats are scientific instruments that set the depth at which they drift with the currents, by calibrating their density so they are neutrally buoyant with the surrounding water at that layer. The first floats were built in 1955 and were ballasted with a fixed mass to descend to a target depth [184]. At the end of the mission, this mass would be released to cause the float to ascend to the surface for recovery. In the 1990s, the first profiling floats were developed that could repeatedly vary the depth they were at using a volume changer (VOCHA). Floats have long been used to measure subsurface currents by descending to a set depth, remaining there for days to months before surfacing. The trajectory of the float during this time matches that of a fluid parcel, and so can be used to infer the current velocity at that level.

Besides drifting with the currents to measure it, floats can also exploit the currents to move in 3D. By diving to different depths, where the current points in different horizontal directions across the various layers, the float can choose which heading it is carried along. Recently, starting from 2011 [181], a number of works have examined being able to perform horizontal maneuvers using floats.

In this section, recent works on float motion planning are reviewed, and a 
classification of these works is presented.

\subsubsection{Controllability results}

The first paper to examine float motion planning was that of Smith and Dunbabin [181]. Before developing algorithms for computing trajectories to goal locations, the authors first wished to establish whether it was even feasible for the float to move to any goal location. If not, the use of floats could be dismissed as having limited value.

In control system theory, this ability is a formal concept known as global controllability. A control system is defined by a function [110]

$$
\dot{\mathbf{x}}=\frac{d \mathbf{x}}{d t}=f(\mathbf{x}, \mathbf{u})
$$

relating the position of the system $\mathbf{x} \in \mathbb{R}^{n}$, or other manifold, to the control actions $\mathbf{u}$ that it takes at each time $t$, where there may be some constraints on the values of the control actions. A point $\mathbf{x}_{1}$ is reachable from $\mathbf{x}_{0}$ if an action trajectory $\widetilde{\mathbf{u}}(t)$ exists satisfying the constraints, such that the resulting state trajectory $\widetilde{\mathbf{x}}(t)$ beginning at $\mathbf{x}_{0}$ passes through $\mathbf{x}_{1}$ after finite time. A system is said to be controllable from point $\mathbf{x}_{0}$ if it can reach any position $\mathbf{x} \in \mathbb{R}^{n}$, and globally controllable if it is controllable from every point $\mathbf{x}_{0} \in \mathbb{R}^{n}[39]$.

By modelling the float as a driftless control affine nonlinear control system [39], Smith and Dunbabin [181] were able to find necessary and sufficient underwater current conditions for global controllability. Their theorem, rewritten for this thesis, is presented below. For clarity, the theorem is preceded by definitions and lemmas from computational geometry, where the expressions for convex hull and positively spanning are reproduced from Mason [124], and the definition for compactness makes use of the Heine-Borel theorem [162].

Definition 5.1.1. Convex hull: the convex hull of a set of vectors $\mathcal{G}=\left\{\mathbf{v}_{i}\right\}$ in $\mathbb{R}^{n}$ is the set of all convex combinations of the elements of $\mathcal{G}, \operatorname{conv}(\mathcal{G})=\left\{\sum_{i=1}^{|\mathcal{G}|} k_{i} \mathbf{v}_{i} \mid k_{i} \geq\right.$ $\left.0, \sum_{i=1}^{|\mathcal{G}|} k_{i}=1\right\}$.

Definition 5.1.2. Positive (linear) span: the positive span of a set of vectors $\mathcal{G}=\left\{\mathbf{v}_{i}\right\}$ in $\mathbb{R}^{n}$ is the set of combinations of its elements given by $\operatorname{pos}(\mathcal{G})=$ $\left\{\sum_{i=1}^{|\mathcal{G}|} k_{i} \mathbf{v}_{i} \mid k_{i} \geq 0\right\}$. The set is said to positively span $\mathbb{R}^{n}$ if $\mathcal{G}=\mathbb{R}^{n}$.

Definition 5.1.3. Compactness: a subset $\mathcal{G}=\left\{\mathbf{v}_{i}\right\}$ of $\mathbb{R}^{n}$ is said to be compact if it is closed and bounded. By bounded it is meant there exists a scalar $r$ such that 
$\forall \mathbf{v}_{i} \in \mathcal{G},\left\|\mathbf{v}_{i}\right\|<r . \mathcal{G}$ is closed if every limit point of $\mathcal{G}$ is an element in $\mathcal{G}$, where a point $\mathbf{p}$ is a limit point of $\mathcal{G}$ if every neighbourhood of $\mathbf{p}$ contains another point not equal to $\mathbf{p}$ that is also contained in $\mathcal{G}$.

Lemma 5.1.1. Every finite subset of $\mathbb{R}^{n}$ is compact. This result is stated in Rudin [163].

Lemma 5.1.2. If a set $\mathcal{G} \subseteq \mathbb{R}^{n}$ is compact, then its convex hull $\operatorname{conv}(\mathcal{G})$ is also compact. A proof of this is given in Leonard and Lewis [83].

Theorem 5.1.1. In the $3 D$ space $\mathbb{R}^{3}$, let a float be able to move instantaneously at each horizontal position $\mathbf{x}=(x, y)$ to $m$ predetermined heights, $Z=\left\{z_{1}, z_{2}, \ldots, z_{m}\right\}$. At each height the horizontal velocity of the float is given by the current velocity field value there, $\mathbf{v}_{U}(x, y, z, t)=\left[u_{U}(x, y, z, t) v_{U}(x, y, z, t)\right]^{T}$, where $t$ is time. The motion of the float can then be represented by the driftless control affine system

$$
\dot{\mathbf{x}}=\sum_{i=1}^{m} \mathbf{g}_{i}(\mathbf{x}, t) u_{i}
$$

where each vector field $\mathbf{g}_{i}(\mathbf{x}, t)=\mathbf{v}_{U}\left(x, y, z_{i}, t\right)$ is the current velocity at height $z_{i}$. The action $\mathbf{u}(t) \in U \subseteq \mathbb{R}^{m}$ represents the choice of height at this particular time, where $u_{i}=1$ if $z_{i}$ has been selected or $u_{i}=0$. The control set is thus

$$
U=\left\{\left[\begin{array}{llll}
1 & 0 & \ldots & 0
\end{array}\right]^{T},\left[\begin{array}{llll}
0 & 1 & \ldots & 0
\end{array}\right]^{T}, \ldots\left[\begin{array}{llll}
0 & 0 & \ldots & 1
\end{array}\right]^{T}\right\}
$$

The system is globally controllable in $\mathbb{R}^{2}$ if and only if the convex hull of the set of selectable current velocities at each point $\mathbf{x} \in \mathbb{R}^{2}$ and time $t$,

$$
\mathcal{G}(\mathbf{x}, t)=\left\{\mathbf{g}_{1}(\mathbf{x}, t), \mathbf{g}_{2}(\mathbf{x}, t), \ldots, \mathbf{g}_{m}(\mathbf{x}, t)\right\}
$$

is compact and contains the origin.

This can be proven as follows. Now, it is known from Theorem 5.5 of Mason [125] that the origin is in the interior of the convex hull of a set of vectors in $\mathbb{R}^{n}$ if and only if that set of vectors positively spans the entire space $\mathbb{R}^{n}$, giving the forward direction proof. In the reverse direction, this Theorem means that the convex hull of a set of vectors that positively spans the space it must contain the origin. Since these vectors, the current velocities, are generated from a finite number of depth choices, the convex hull is al compact, completing the proof. This allows us to present the following corollary: 
Corollary 5.1.2.1. The system described in Theorem 5.1.1 is globally controllable in $\mathbb{R}^{2}$ if and only if the set of selectable current velocities at each point $\mathbf{x} \in \mathbb{R}^{2}$ and time $t$ positively spans $\mathbb{R}^{2}$.

A test to check that the vectors satisfy this criterion is to lay out all the vectors so that they originate at a common point. The set of current vectors $\mathcal{G}(\mathbf{x}, t)$ at $\mathbf{x}$ positively span $\mathbb{R}^{2}$ if and only if the largest angle between adjacent vectors is less than $\pi$,

$$
\max _{i, j} \theta_{i, j}(\mathbf{x})<\pi
$$

where $\theta_{i, j}(\mathbf{x})$ is the angle between adjacent current vectors $\mathbf{g}_{i}(\mathbf{x}, t), \mathbf{g}_{j}(\mathbf{x}, t)[130]$. By adjacent it is not meant that the current vectors come from successive depths, but that the vectors are angularly adjacent when arranged about a common point.

The controllability criterion was applied by Smith and Dunbabin [181] to realistic current velocities generated by a numerical ocean model of southern Californian coast. The region was divided into a grid. Each point was marked red if the current over a depth range of 0 to $125 \mathrm{~m}$ positively spanned the plane or blue if not. Figure 5.2 shows the output of this colouring, where each graph evaluates the current at 0,12 and 24 hours. Most of the points satisfy the Theorem. Large contiguous areas, such as in the northwest corner and near the coast, consist of these points that remain red over the entire time period, indicating that a float deployed anywhere within one of these regions could move to any other goal location within the are, and float motion control was declared worth pursuing.

\subsubsection{Well-behaved current-based schemes}

A number of float control strategies have since been based on the current being "well-behaved", that is, satisfying the controllability criterion of Theorem 5.1.1.

Jouffroy et al. [93] propose a controller to enable a float to find its way to the goal location. Time is broken up into timesteps, where the profiler can move to any of $m$ fixed depths in the next interval. The current at each layer causes the device to be carried some incremental horizontal displacement, so if $\mathbf{X}(k)$ is its horizontal position at timestep $k$, its position at timestep $k+1$ is given by $\mathbf{X}(k+1)=\mathbf{X}(k)+\mathbf{X}_{\mathrm{c}}(k)$. The incremental displacement $\mathbf{X}_{\mathrm{c}}(k) \in\left\{X_{\mathrm{c} 1}(k), X_{\mathrm{c} 2}(k), \ldots, X_{\mathrm{c} m}(k)\right\}$, where $X_{\mathrm{c} i}$ is the displacement for drifting along layer $i$. The float is assumed to be able to predict the horizontal displacement. The controller selects the depth for the next timestep, the incremental displacement vector of which makes the smallest angle with the line of 

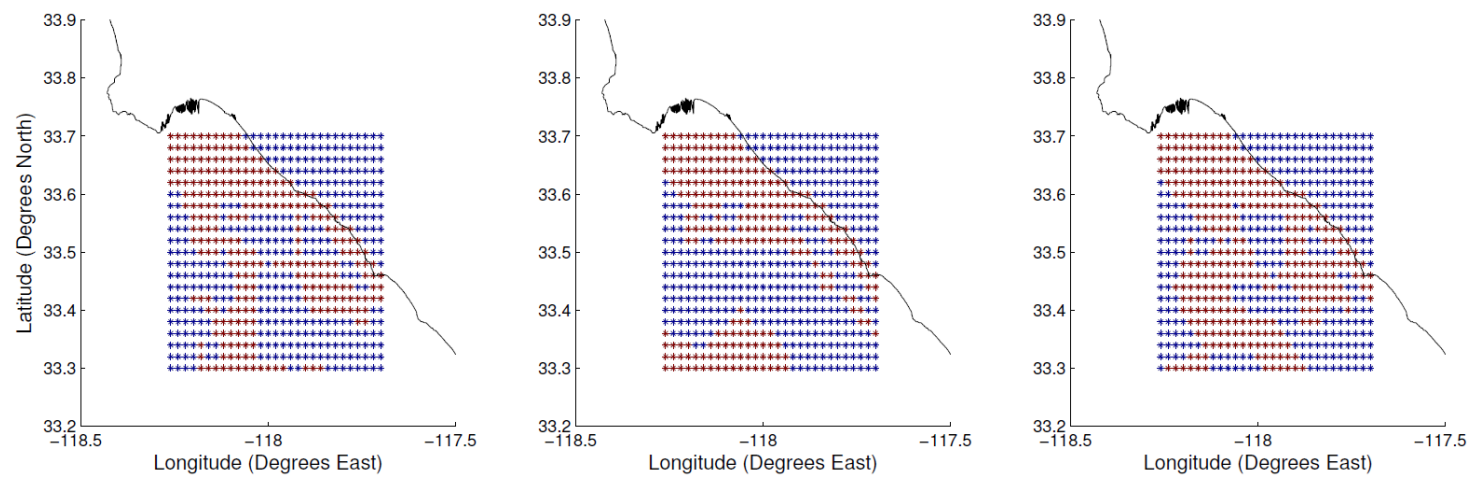

Figure 5.2: Current off the coast of southern California, assessed according to the controllability criterion in Theorem 5.1.1. Points are marked red if the current between $0-125 \mathrm{~m}$ depths satisfied the Theorem, positively spanning the plane, or blue if not. Going rightwards, the current 0, 12 and 24 hours after the time in the leftmost graph has been used in each figure. Image from Smith and Dunbabin [180].

sight vector extending from the current position of the float to the goal. It is shown that as long as the incremental displacement vectors at every visited point positively span the plane, this algorithm will enable the float to converge in finite time to a neighbourhood around the goal.

A similar algorithm is employed in Molchanov et al. [130], in combination with an artificial potential field controller, for positioning multi-node systems. At present, floats are recovered and deployed by ships [160]. The cost to run vessels is very high. Costs could be reduced if floats used the currents to converge to one horizontal position at the end of their mission or spread out from an initial location. Less time would then be needed to perform these operations. Floats could be picked up at one location by a ship, rather than the vessel having to spend additional time travelling between each device. Similarly, where floats need to be deployed over a wide area, the ship only has to release the nodes at one location and the floats will spread out by themselves. A node movement scheme to achieve these goals, here termed aggregation and dispersion respectively, is proposed. No guarantee is provided for the time needed for the float to arrive at the destination, nor is the vertical distance profiled by the float and the energy expended while doing so considered.

Aggregation and dispersion for fully actuated robots can be achieved by utilizing artificial potentials [77]. The use of artificial potential fields for formation control is inspired by flocking behaviour. Herds, schools of fish and flocks of birds are able to perform complex group maneuvers despite each animal making no obvious use of communications, and so having to make individual decisions only from what 
they can directly perceive with their eyes, namely their spacing from surrounding creatures. Scientists have been able to produce flock-like behaviour using rules that respect these constraints, where animals are attracted to (move towards) distant neighbours, are repulsed (move away) from neighbours that are too close and steer towards the velocity heading of their neighbours [156], and it is now suggested by biologists that this is how herds organize themselves [115].

In the artificial potential field approach, these forces are replicated by defining a unique set of potential fields associated with each node. A control force on the node is specified that is minus the gradient of the sum of all the potentials or, as the gradient operator is distributive over addition, the negative sum of the gradient of each potential. Each potential is a function of the distance from this agent to another node or reference point. The gradient of a potential is thus always along the straight line between the node and its neighbour, either pointing away from or towards the neighbour, resulting in the force on the device being the sum of attractive or repulsive forces respectively. The node is accelerated in the direction of the force and, due to the resulting change in positions of the nodes, the force is also updated. Eventually the vehicles converge to a formation where the total potential or energy is minimized [115]. The formation depends on how the attractive or repulsive forces are specified, and artificial potential fields have been defined for expanding and contracting formations of underwater gliders [61], and evenly spreading out wireless sensor networks [79].

In Molchanov et al. [130], an artificial potential controller specifies horizontal directions that the floats should try to move along to aggregate together or disperse. For aggregation, an attractive force is defined between each node with each another and the pick-up point, which, if each node was fully actuated and could move in the direction of the resultant force, would lead to the network converging to the target point. For dispersion, other nodes exert a repulsive force and nodes are attracted to a reference point so that, while spread out, they remain inside the region of interest.

Profiling floats are only vertically actuated however. Their horizontal velocity is equal to that of the currents. While a float can change its horizontal velocity indirectly, by descending or ascending to different depths, it may not be able to move instantaneously in any direction if no current along the profile points in that direction. It is known, though, that if the current choices at every visited point positively span the 2D plane, then the depth controller in Jouffroy et al. [93] allows a profiler to converge to a horizontal goal position. Molchanov et al. [130] utilize this depth controller to track on to the high-level direction set by the artificial potential 
controller. The potential field controller is run at a central location, as forces on each node are calculated between every float in the network. The high-level direction control is communicated back to each float. The float then descends to a fixed number of set depths to measure the current there. Depths $\{0,10,30,75,200,400\} \mathrm{m}$ are chosen in the validation scenario conducted. From this set, the devices descends to the depth where the current makes the smallest angle with the direction vector is selected.

The scheme is evaluated in simulation, employing the numerical ocean model output as the current in the scenario. The strategy is simulated using an idealized float, which has infinite vertical speed, and a float that can move up to a more typical maximum vertical actuated speed of $0.3 \mathrm{~ms}^{-1}$, resulting in profiling and moving between depths taking significant time. Figure 5.3 shows the aggregation performance over time. The aggregation metric is plotted, defined as the normalized pairwise potential energy of points, where a higher value indicates the nodes are more clustered together at a single location. The scheme performs much worse with the more realistic float model. A problem with the results is that the outcome of no depth control or even a naive scheme are not presented, so it is difficult to determine how much of an improvement the strategy provides. For example, each node just picking random depths to move at would be expected to disperse the network as well. All the additional control in the scheme of [130] scheme may not provide much benefit. Again, as for [93], the scheme is only evaluated by its ability to satisfy the positioning goal, in this case by the coverage / aggregation metric. The energy usage of the nodes in mobility is not considered. This is a common feature shared among all the schemes we examined. To avoid repetition of this observation in the rest of this survey, the metrics we describe as being used to evaluate schemes should be taken as a complete accounting of all the metrics employed in the work being discussed.

The controllability criterion of Theorem 5.1.1 is employed in Smith and Huynh [182] to search for the most feasible horizontal path a profiling float can take to a goal. Their strategy requires past current data over the region to be available; in their case this is provided by a numerical ocean model. The 2D plane is divided up into a grid. The current at each point at each recorded time is then assessed for the proportion of times where the currents along the profile did not positively span the $2 \mathrm{D}$ plane. This number is recorded at each grid point. Figure 5.4 shows the ensuing controllability map. An $A^{*}$ search [135] is then performed to find the optimal sequence of adjacent grid points from the start to goal location that minimizes the sum of the points' 


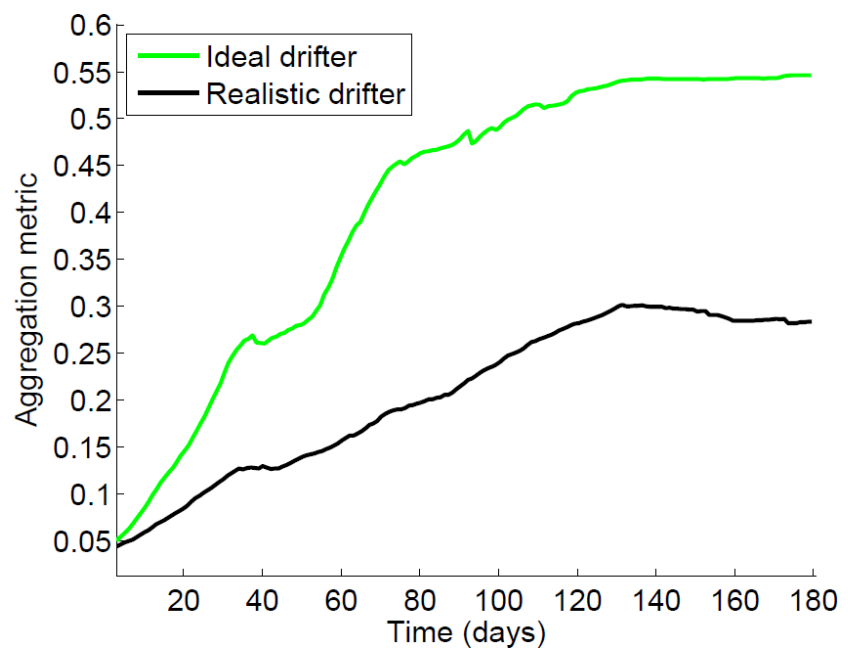

Figure 5.3: Float network aggregation performance over time of Molchanov et al.'s scheme [130], where an idealized and a more realistic model, having finite vertical velocity, of the float is considered. Image from Molchanov et al [130].

non-spanning proportion. The nodes comprise a contiguous region of points that historically have been found to positively span the 2D plane. Thus, the region is highly likely to be globally controllable and a path in this region exists to the goal location. A finite horizon model-predictive controller is then used to find the depth changes needed to move between the points.

Several simulations show that the controller is able to get the float close to goal positions $\approx 2$ nautical miles from its starting point, if by circuitous paths.

\subsubsection{Selective Tidal-Stream Transport (STST)-based schemes}

Since current velocity tends to decrease with depth, floats can effectively remain stationary by descending to great depths. In shallow waters, the float can embed itself in the seabed, such as with pikes attached to the bottom of the device in the Arvor-C float [15]. One way to reach a horizontal position then is for a node to only drift with the current when, the current being time-varying, it is pointing in the direction of the destination, remaining fixed otherwise.

Such schemes are categorized here as Selective Tidal-Stream Transport (STST)based strategies, after the behaviour in animals [66]. Some creatures similarly burrow themselves into the seabed to wait for the correct current conditions. For instance, blue crab postlarvae reduce the energy they need to move upshore by swimming only during the flood tide and descend to the bottom on the ebb tide. 

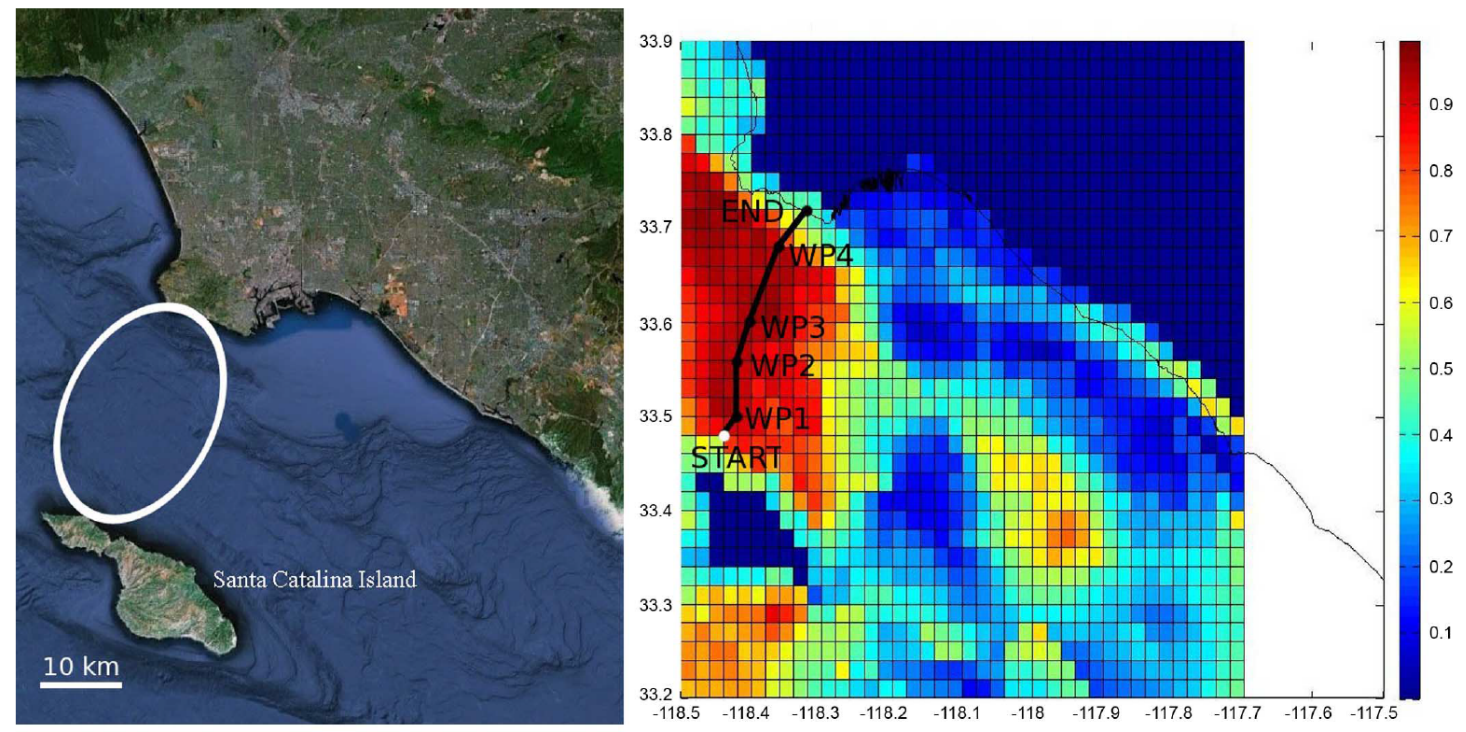

Figure 5.4: Controllability map of San Pedro Bay off the coast of southern California. The colourmap values are the proportion of times the current at the grid point positively spanned the $2 \mathrm{D}$ plane. Also shown, in black, is the path with the highest likelihood of execution found by the $\mathrm{A}^{*}$ search. Image from [182].

This idea is utilized by the controller in Jouffroy et al. [93] for float path planning in coastal waters, where the profiler can latch onto the seafloor. The float is given two choices for the next timestep, to move to the seabed or the surface. The transit time between depths is ignored, so if the seabed is selected the horizontal position of the float is unchanged. In the other case, the float is carried by the surface currents some horizontal displacement $\mathbf{X}_{\mathrm{c}}(k)$ from its last position $\mathbf{X}(k)$ by the start of the next timestep. The controller only chooses to surface if the current is in the general direction of the goal, defined as the angle between the line connecting $\mathbf{X}(k)$ to $\mathbf{X}(k)+\mathbf{X}_{\mathrm{c}}(k)$ and the line extending from $\mathbf{X}(k)$ to the target position being less than $\theta_{\mathrm{L}}$. A proof is given, showing that as long as the current at each visited position eventually points in the general direction of the goal and is sufficiently strong, the float using this strategy will converge to a neighbourhood around the target position.

The controller was tested in a simulated scenario. The profiler was tasked with reaching three horizontal points. A time record of the surface current measured at a single point off the coast of Germany during 2006 was used for the current velocity at each timestep. The distance from the seabed to the depth where the current was measured is only $8 \mathrm{~m}$, meaning that the float can rapidly affix itself to the ground. In coastal areas, the tides add a periodic time-varying component to the currents. The trajectory of the float is displayed in Figure 5.5. The profiler moves on to the next 
waypoint, pink, once it gets within a circular region of the preceding goal location. The results indicate that near the shore, floats can move horizontally to desired locations using the currents when they can anchor themselves.

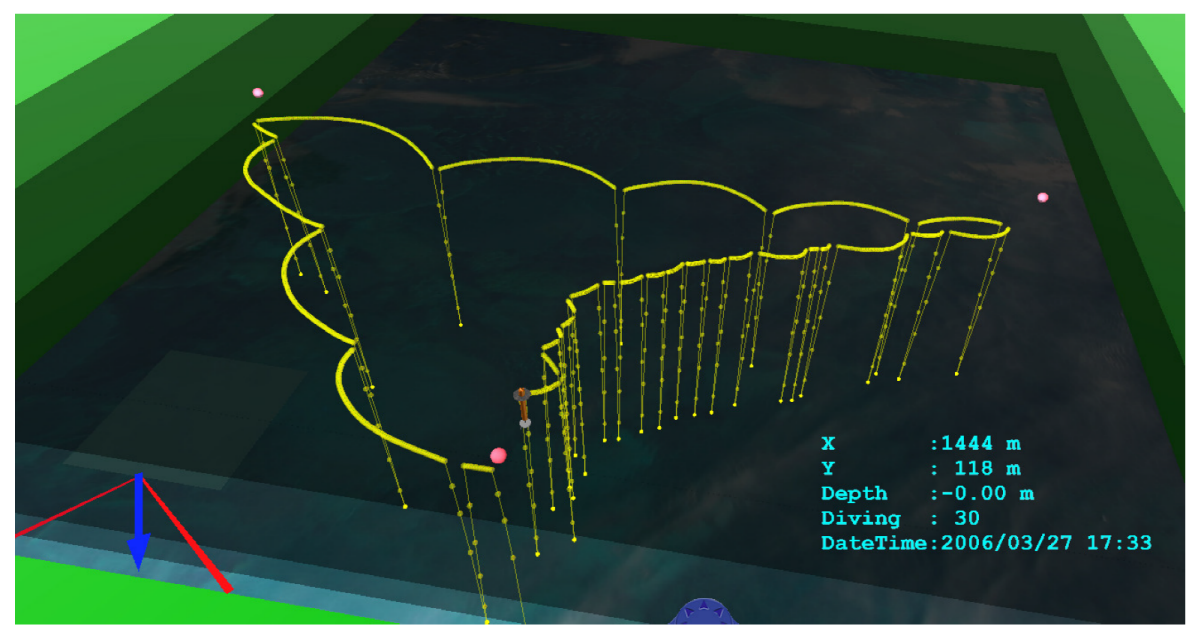

Figure 5.5: Trajectory of the float using the controller in Jouffroy et al. [93] to reach three waypoints (pink) by drifting with the surface current when it is pointing towards the goal and remaining stationary on the seabed at other times. Controller is being tested in a simulated environment, where real-life current data measured off Germany from 2006 is employed. Image from Jouffroy et al [93].

\subsubsection{General current schemes}

The previous categories of float motion planning strategies depend for their success on the current meeting certain criteria, whether it be that the current drops off to near-zero with depth or controllability is satisfied according to Theorem 5.1.1. In this section, schemes that rely on no current assumptions are surveyed.

Dahl et al. [42] investigate the problem of 2D aggregation and dispersion of the Argo float array, seen previously in Molchanov et al [130]. In this case, the authors propose a pregenerative constant depth and greedy scheme to achieve these objectives. An accurate current prediction is assumed to be available. Aggregation is expressed by the mean sum of each float's distance to the collection point, where a lower value is therefore preferable.

In the constant-depth algorithm, each float remains at a fixed (but possibly different between profilers) depth over the entire mission. The combination of depths that out of $m$ discrete values minimizes the objective function at the end of the mission is found. Beginning with a default combination, the end positions of each float are found by numerical integration, employing the predicted current as the 
drifting velocity of the float at each point. The value of the objective function can then be computed. The depth of a profiler is varied with, of the $m$ choices, the depth that results in the best value of the cost function being retained. This is repeated for each float until no depth assignment is changed in one complete pass.

In the greedy scheme, the depths of the nodes are re-evaluated in-mission at regular time intervals. At each timestep, the centralized algorithm iterates through each node. The state of the network one interval later is forward simulated $m$ times, where the profiler is at one of the $m$ depth choices and the other floats remain at their current depth if not already set. Floats are assumed to be be able to change depths instantaneously. The depth that had the lowest network objective function is chosen for the node to drift at in the next timestep. This process is then repeated for every float. The scheme is labelled greedy as it selects the best action based on the predicted outcome in the next timestep only. A short-sighted approach is necessary, as the number of node depth combinations grows exponentially with the number of future timesteps being considered. It is computationally infeasible to evaluate them all.

The algorithms are simulated in 30 day missions with 5 day timesteps, where the current is given by a numerical ocean model. The schemes are compared to leaving the ARGO floats to repeat their 10-day cycles. Vastly improved performance over this baseline measure is reported, with objective function values twice or three times that of no control.

\subsubsection{Current prediction-based schemes}

It was seen that several motion planning algorithms used current predictions to determine the changes in depth of floats $[42,181,182]$. The accuracy of these predictions is unreliable, however. For the specific region and mission time over which the network is deployed a numerical ocean model must be used. The output of these programs is very low-resolution, giving the predicted current velocity at kms horizontal spacings, a number of discrete depths and hourly intervals. Moreover, the prediction is typically only based on up-to-date measurements of the surface current by radar or satellite. For the deep water conditions that are substituted into the equations of motion, the model relies on historical data, namely, casts of previous cruises, perhaps years back, of the underwater currents and seawater properties. The current prediction can therefore be considered reasonable for near-surface current velocities, but inaccurate for deeper currents [179]. It is also counter-intuitive to suggest that floats, deployed to gather data on currents [25,160], should know exactly 
what the current is beforehand in order to accomplish their mission.

As an example of how much further current predictions need to go before they can be effective in motion planning, Smith et al. [179] investigated utilizing current predictions to improve waypoint reaching for gliders. At present, a glider computes an average current velocity from its displacement between surfacings and the location it would have surfaced at if there was no current. The glider then subtracts the heading of this current from the direction to its next waypoint, in order to compute the actuated horizontal velocity it is to move at. As the current actually varies over its trajectory, the vehicle does not end up at its intended position.

Smith et al. [179] predict the position that the glider will end up by this dead reckoning approach. The trajectory is discretized into $30 \mathrm{~s}$ intervals, where the vehicle's constant velocity during this segment and the current velocity are integrated to get the glider's incremental displacement. The predicted current is provided by ROMS (Regional Ocean Model System), a numerical ocean model that is configured to provide the current hourly, at $2.2 \mathrm{~km}$ horizontal resolution and 22 depths unevenly spaced (mainly near the surface) between $0 \mathrm{~m}$ and $2000 \mathrm{~m}$. The predicted current along each part of the trajectory is the average value of the eight nearest ROMS grid points. If the glider ends up within $100 \mathrm{~m}$ of the waypoint, the dead reckoning trajectory is retained. Otherwise, the velocities in the intervals are iterated upon until a trajectory is generated that is predicted to end up within. The glider executes this trajectory. While the course the glider followed should have put the device within $100 \mathrm{~m}$ of the waypoint $100 \mathrm{~m}$ of the waypoint is the predicted currents were exact, this was not the case. Over 10 experimental trials, using this planner, the glider ended up on average $0.7 \mathrm{~km}$ away from the waypoint at $1.5 \mathrm{~km}$ distance, or an error of $47 \%$. This is likely owing to the interpolated current being inaccurate.

A node movement scheme designed to give desired trajectories with perfect current predictions is probably not going to work in practise, where this level of forecasting has not been reached. In this thesis, we investigate what coverage performance can be achieved by a scheme that does not rely on current predictions, but rather currents measured in-situ.

\subsubsection{In other fields}

In our readings, we also came across multiple works on deployment and movement schemes for nodes in land-based WSNs [207], UWSNs using AUVs [119,120], and 3D UWSNs where the nodes can only move up and down [50], because the sensors are moored to the seabed and can adjust the length of their mooring line using a winch, 
to improve coverage and connectivity. While being related to our 3D float-based, underwater node movement problem, this resemblance was only superficial, and we could find little in the solutions proposed in these fields that could be applied to our topic. Terrestrial mobile nodes and AUVs can move omnidirectionally, whereas floats can only move up and down. Moored nodes also can only vary their depth but their horizontal position is fixed and the network does not become more dispersed over time.

\subsubsection{Summary}

This section summarizes our survey, by considering existing works as a whole. We saw that existing float motion planning schemes can be categorized based on the assumed current behaviour into well-behaved current-based schemes [93,130,182] and general current-based schemes [42]. Two main approaches for exploiting the current for horizontal mobility were identified: selective tidal-stream transport [93], where nodes descend into deeper, near stationary water until a current in the upper layer changes direction and allows the node to make progress towards its target location, and current prediction-based schemes $[42,181,182]$. In the second category, a forecast of the currents in the region is available to the nodes. An algorithm uses this data to generate a sequence of depth changes that the float can follow to move to its destination. A problem with schemes that rely on current predictions is that often forecasts aren't accurate, resulting in the position that the float actually ends up being quite different from the computed location. Smith et al. [179] were cited, who found the distance between the target position, which a glider should end up based on current predictions, and its actual end point was $47 \%$ of the start point-target point distance.

The roles of float motion planning schemes have so far been limited to trajectory planning for single floats $[93,181,182]$ or formation control of widely-spaced float arrays $[42,130]$, where in both cases the aim is to achieve a $2 \mathrm{D}$ position or formation. As a result, these schemes cannot be directly applied to our problem of guiding a float to the location of a coverage or connectivity hole in 3D. The final depth reached by the float is not controlled, so while the node may arrive at the desired horizontal co-ordinates, it is at the incorrect depth to cover the hole.

The proposed schemes are greedy $[42,93,130]$ and/or assume floats can move with infinite vertical velocity $[42,130,181,182]$. A common greedy strategy has been a sector-of-sight approach, in which nodes choose an immediate depth to drift along where the current points in the closest direction towards the destination $[93,130]$. 
When realistic vertical actuated velocities are employed, the performances of these scheme can be significantly impacted, for the worse as shown in Figure 5.5. It is assumed that the infinite vertical velocity simplification is also the reason why details of how information needed by the schemes are disseminated are not given. The nodes can surface at any time and use RF communications to obtain their movement goal directly from the centralized controller, as in [130], and receive current forecasts [42].

The finite profiling velocity of the float cannot be neglected in our 3D coverage problem. This is shown by considering what would occur if a scheme was designed assuming that floats can move instantaneously between layers. Then the 2D path planning strategies we surveyed could be used to move profilers on to 3D positions associated with coverage holes: by executing the strategy until the node is at the horizontal co-ordinates of the target, then moving directly to the target depth to be at its exact location. In reality though, the float must traverse the intervening depths between that specified by the $2 \mathrm{D}$ controller and the depth of the goal. The horizontal displacement from the target that develops during this period can be significant. Consider a typical scenario [205] where the sensing range is $100 \mathrm{~m}$ and the current velocity varies linearly with depth as for Figure 3.15, from a surface speed of $0.39 \mathrm{~ms}^{-1}$ to a speed of $0.22 \mathrm{~ms}^{-1}$ at $500 \mathrm{~m}$ depth, the vertical span of the RoI (Figure 4.3). Even travelling at maximum speed $\left(0.3 \mathrm{~ms}^{-1}\right)$ to minimize the horizontal displacement, in the worst-case scenario where the goal is at surface depth and the node is located at $500 \mathrm{~m}$ depth, the float will be displaced horizontally by $510 \mathrm{~m}$. This is unacceptable since it puts the node out of sensing range of the point it is supposed to be covering. For this reason, we propose a 3D float movement scheme that takes into account the finite velocity of the float.

\subsection{Scheme 1: our novel 3D UWSN coverage ex- tension float movement scheme}

In this section, we define our float node movement scheme, Scheme 1. The node movement scheme specifies the vertical actuated velocities of each float

$$
S=\left\{w_{1 C}(t), w_{2 C}(t), \ldots, w_{n C}(t)\right\}
$$

in a UWSN consisting of $n$ profiling floats assigned with a general monitoring task of sensing a $3 \mathrm{D}$ region of interest $(\mathrm{RoI}) \mathcal{R} \subseteq \mathbb{R}^{3}$ over the mission duration $\mathcal{T}=\left[t_{\mathrm{i}}, t_{\mathrm{f}}\right] \subseteq \mathbb{R}$, with the goal of maximizing coverage. In real terms, the area could comprise an oil and gas field, a shipwreck (salvage site) or a harbour entrance [41]. 
The principal novelty of our scheme is that it seeks to maximize coverage of a 3D RoI.

Improving coverage through the use of mobile nodes has been a common aim in many WSN [207] and UWSN works $[42,121,130]$. Coverage is considered one of the most important measures of the quality of service (QoS) provided by a WSN, as it evaluates how well the deployed sensors observe the RoI [49]. In this work, we employ the definition of coverage utilized in [121] which assumes a binary disk sensing model [65]. All points within distance $R_{S}$, known as the sensing range, of a float are covered by that node. The area coverage at time $t$ of the network itself is defined as the volume of all points within the RoI that are covered by at least one node

$$
C(t)=\left|\mathcal{R} \cap\left(\bigcup_{i=1}^{n} C_{i}(t)\right)\right|
$$

where $|B|$ is the volume of set $B$, and

$$
C_{i}(t)=\left\{\left[\begin{array}{lll}
x & y & z
\end{array}\right]^{T} \mid\left(x-x_{i}(t)\right)^{2}+\left(y-y_{i}(t)\right)^{2}+\left(z-z_{i}(t)\right)^{2} \leq R_{S}^{2}\right\}
$$

is the set of all points in $\mathbb{R}^{3}$ covered by float $i$ of $n$ nodes at position $\mathbf{x}_{i}$ at time t. This can be normalized to a unitless value between 0 (no coverage) and 1 (full coverage), the coverage proportion specifying the fraction of the RoI being covered, by dividing by the measure of the RoI

$$
C_{p}(t)=\frac{C(t)}{|\mathcal{R}|}
$$

Formally, we define the goal of our scheme as maximizing the network's coverage over the length of the mission or its cumulative area coverage proportion

$$
\arg \max _{w_{1 C}(t), w_{2 C}(t), \ldots w_{n C}(t)} \int_{t_{i}}^{t_{f}} C_{p}(t) d t
$$

We saw in our survey that a class of 2D float path-planning strategies exist that rely on the ocean environment being $2 \mathrm{D}$ controllable. These were termed well-behaved current-based schemes by us. Our scheme can be considered a type of well-behaved current-based scheme. It relies on the environment being 3D controllable for finite-velocity profiling floats.

As each float can be piloted to any location, they can be moved to points in a structured lattice that fully covers the RoI. Since the shape of the RoI remains invariant, it can be fully covered by an arrangement or lattice of $m$ nodes, preferably 
minimal so $n \gg m$, having fixed positions $\left\{\mathbf{x}_{1}, \mathbf{x}_{2}, \ldots, \mathbf{x}_{m}\right\}$ relative to the RoI. In well-behaved currents, floats can be moved on to the lattice points. However, full coverage, which is given by full occupancy of the lattice at the time instant, is unlikely to be achieved. Firstly, the trajectory that the profiler needs to take to end up at the lattice point is dependent on the currents. Nodes throughout the network may not be able to arrive at their locations at the same time. Secondly, as soon as a float manages to arrive at the lattice point, it is immediately carried away at the speed of the local horizontal current.

In this work, we come up with the original idea of achieving 3D coverage in float-based UWSNs using float trains. The above problems can be solved and full coverage obtained by utilizing cyclic streams of floats that arrive one after another at a point with no time between arrivals and departures so the point can be continuously covered.

We develop a float train-based float movement scheme in Scheme 1. The scheme consists of two main elements: a one-off network-wide target assignment routine and a lightweight trajectory generator, implemented on each individual node. The scheme is outlined. Immediately after deployment, while the nodes are on the surface for network-wide RF communications, the $n$ network nodes are evenly assigned one of $m$ lattices points to repeatedly reach. The set of all nodes tasked with passing through or linked with a particular lattice point is non-empty and consists of multiple floats. Each node computes and executes a time-limited trajectory aiming on its way to travel as close as possible to its assigned lattice point. Upon completion of its moves, the float determines and follows a trajectory to the lattice point from its new position, repeating these steps until the mission end.

For now, in this initial scheme we are not overly concerned with mechanisms for ensuring the arrival times of the nodes at each lattice point are evenly spread out. The scheme relies on natural variation in the durations of the found trajectories to space out the arrivals of floats at each lattice point, so that the nodes pass the point not at the same time but one after another to cover the region around the point in an unbroken succession. The advantage of this approach is that each node can compute its trajectory without reference to other profilers, leading to a communications trajectory generation algorithm.

Implementation details of the trajectory generation and target assignment routines are given in Sections 5.4-5.5. 


\subsection{Conditions for 3D controllability}

Our node movement scheme relies on the current being well-behaved, which leads floats to be $3 \mathrm{D}$ controllable in the environment. We now state sufficient conditions for $3 \mathrm{D}$ controllability. These conditions can be viewed as assumptions made by our scheme.

Theorem 5.3.1. Given the following assumptions, a profiling float is controllable in $\mathbb{R}^{3} \backslash z<z_{\Omega}$, where $z$ is the sea surface height. The value $z=0$ lies on the surface, and negative values of $z$ correspond to a lower depth. The variable $z_{\Omega}<0$ is the minimum depth we restrict the float to be able to descend to.

1. The velocity of the float is

$$
\begin{aligned}
\frac{d \mathbf{x}}{d t} & =\mathbf{v}(t) \\
& =\mathbf{v}_{C}(t)+\mathbf{v}_{\mathbf{U}}(t, \mathbf{x}) \\
& =\left[\begin{array}{lll}
0 & 0 & w_{C}(t)
\end{array}\right]^{T}+\left[\begin{array}{lll}
u_{U}(\mathbf{x}, t) & v_{U}(\mathbf{x}, t) & w_{U}(\mathbf{x}, t)
\end{array}\right]^{T}
\end{aligned}
$$

where $\mathbf{x}$ is the float's position, $\mathbf{v}_{U}$ is the current velocity at this position, and $\mathbf{v}_{\mathrm{C}}$ is the float's self-propelled velocity. This model, where a device's total velocity is the sum of the ambient velocity field value and its self-propelled velocity has been frequently employed to describe different drifting objects, from floats [181], drogued drifters [130], underwater vehicles [121], hot air balloons [60] to general drifting objects [94]. In this thesis, the two velocities are labelled $\mathrm{U}_{\mathrm{U}}$ and $\mathrm{C}_{\mathrm{C}}$ after Luo et al. [121], where they denote uncontrollable and controllable respectively.

2. The current velocity is non-time-varying and non-horizontally spatially varying,

$$
\begin{aligned}
\mathbf{v}_{U}(\mathbf{x}, t) & =\mathbf{v}_{U}(x, y, z, t) \\
& =\mathbf{v}_{U}(z) \\
& =\left[\begin{array}{lll}
u_{U}(z) & v_{U}(z) & 0
\end{array}\right]^{T}
\end{aligned}
$$

3. Between $z_{\mathcal{A}}=0$, the sea surface, and the minimum height $z_{\Omega}$ there exist a sequence of sea level heights

$$
\mathcal{Z}=\left\{z_{1}, z_{2}, z_{3}, \ldots, z_{k+1}\right\}
$$

where $z_{1} \geq z_{2} \ldots \geq z_{k+1}$ such that the horizontal displacements experienced by a float from traversing each adjacent height pair at constant profiling speed 
span the $2 D$ plane. This assumption is analogous to a discretization of the positively spanning current profile assumed by $2 D$ well-behaved current-based float path planning schemes [93, 130,182], which is based on many real-life currents having been observed with this property [42].

Proof. It is sufficient to show that a finite time float trajectory can be found between any two locations $\mathbf{x}_{s}=\left[\begin{array}{lll}x_{s} & y_{s} & z_{s}\end{array}\right]^{T} \in \mathbb{R}^{3} \backslash z<z_{\Omega}$ and $\mathbf{x}_{g}=\left[\begin{array}{lll}x_{g} & y_{g} & z_{g}\end{array}\right]^{T} \in$ $\mathbb{R}^{3} \backslash z<z_{\Omega}$, where $\mathbf{x}_{s}$ is the float's starting position and $\mathbf{x}_{g}$ is its goal.

Consider a trajectory that passes through each height layer in $\mathcal{Z}$ at least once before ending up at the goal location. The trajectory can be represented as

$$
\begin{aligned}
\Gamma & =\left\{z_{s z a}, z_{a z(a+1)}, z_{(a+1) z(a+2)}, \ldots, z_{b z g}\right\} \\
& =\left\{d_{1}, d_{2}, \ldots, d_{l}\right\}
\end{aligned}
$$

where the vertical moves executed by the profiler are broken down into the height layers in $\mathcal{Z}$ visited sequentially by the float, and $z_{a z b}$ denotes a movement between adjacent layers at $z=z_{a}$ and $z=z_{b}$. A total of $l$ such moves are made over the trajectory. The final interval puts the device at the depth of the goal location, so all that remains is to show that the trajectory can achieve the correct horizontal displacement: $\sqrt{\left(x_{g}-x_{s}\right)^{2}+\left(x_{g}-x_{s}\right)^{2}}$.

The horizontal displacement from traversing interval $d_{i}=z_{a z b}$ at vertical velocity

$$
\begin{aligned}
\Delta \mathbf{h}\left(z_{a z b}, w\right) & =\left[\begin{array}{ll}
\Delta x\left(z_{a z b}, w\right) & \Delta y\left(z_{a z b}, w\right)
\end{array}\right]^{T} \\
& =\left[\begin{array}{lll}
\int_{t=0}^{t=\frac{z_{a}-z_{b}}{|w|}} u_{U}\left(z_{b}+|w| t\right) d t & \int_{t=0}^{t=\frac{z_{a}-z_{b}}{|w|}} v_{U}\left(z_{b}+|w| t\right) d t
\end{array}\right]^{T}
\end{aligned}
$$

We observe that the profiling velocity can be moved out of the integral by substituting in $b=z_{b}+|w| t$. This leads to a more convenient expression for the movement experienced by the node as

$$
\begin{aligned}
\Delta \mathbf{h}\left(z_{a z b}, w\right) & =\left[\begin{array}{lll}
\int_{b=z_{b}}^{b=z_{a}} \frac{u_{U}(b)}{|w|} d b & \int_{b=z_{b}}^{b=z_{a}} \frac{v_{U}(b)}{|w|} d b
\end{array}\right]^{T} \\
& =\left[\begin{array}{ll}
\frac{1}{|w|} \int_{b=z_{b}}^{b=z_{a}} u_{U}(b) d b & \frac{1}{|w|} \int_{b=z_{b}}^{b=z_{a}} v_{U}(b) d b
\end{array}\right]^{T} \\
& =\frac{\Delta \mathbf{H}\left(z_{a z b}\right)}{|w|} \\
& =t_{i} \frac{\Delta \mathbf{H}\left(d_{i}\right)}{\left\|d_{i}\right\|}
\end{aligned}
$$

where $t_{i}$ is the time spent traversing the interval and $\left\|d_{i}\right\|=\left|z_{b}-z_{a}\right|$. The vertical velocity of any real float is finite, so there is a minimum value to the traversal 
time given by $\left\|d_{i}\right\| / w_{C \max }$, where $w_{C \max }$ is the maximum profiling speed of the float. The traversal time can be separated into an additional time, $\tau$, and minimum time components:

$$
t_{i}=\tau_{i}+\frac{\left\|d_{i}\right\|}{\left|w_{C \max }\right|}
$$

where the additional time component can take any value in $\tau_{i} \in[0,+\infty)$.

Rewriting the displacement expression using this form, we end up with

$$
\Delta \mathbf{h}\left(z_{a z b}, w\right)=\left(\tau_{i}+\frac{\left\|d_{i}\right\|}{\left|w_{C \max }\right|}\right) \frac{\Delta \mathbf{H}\left(d_{i}\right)}{\left\|d_{i}\right\|}
$$

The term,

$$
\Delta \mathbf{H}\left(z_{a z b}\right)=\left[\int_{b=z_{b}}^{b=z_{a}} u_{U}(b) d b \quad \int_{b=z_{b}}^{b=z_{a}} v_{U}(b) d b\right]^{T}
$$

has units of $\mathrm{m}^{2} \mathrm{~s}^{-1}$, being a velocity integrated with respect to a distance term.

By assumption 3, the set of horizontal displacements (5.16) for each interval positively spans the 2D plane. Positively spanning was defined in Definition 5.1.2 and means that any $2 \mathrm{D}$ vector can be represented by a non-negative linear combination of the $k$ layer horizontal displacement vectors. For all $\mathbf{b} \in \mathbb{R}^{2}$ there exists some $\lambda_{1}, \lambda_{2}, \ldots, \lambda_{k} \geq 0$ such that

$$
\mathbf{b}=\lambda_{1} \frac{\Delta \mathbf{H}\left(z_{1 z 2}\right)}{\left\|z_{1 z 2}\right\|}+\lambda_{2} \frac{\Delta \mathbf{H}\left(z_{2 z 3}\right)}{\left\|z_{2 z 3}\right\|} \ldots+\lambda_{k} \frac{\Delta \mathbf{H}\left(z_{(k-1) z k}\right)}{\left\|z_{(k-1) z k)}\right\|}
$$

The horizontal displacement of the trajectory is

$$
\mathbf{h}(\Gamma)=\sum_{j=1}^{l}\left[\left(\tau_{j}+\frac{\left\|d_{j}\right\|}{\left|w_{C \max }\right|}\right) \frac{\Delta \mathbf{H}\left(d_{j}\right)}{\left\|d_{j}\right\|}\right]
$$

Since each height interval is visited at least once, the displacements experienced in each visit to a specific interval can be collated, which gives

$$
\begin{aligned}
\mathbf{h}(\Gamma)= & \left(\tau\left(z_{s z a}\right)+\frac{\left\|z_{s z a}\right\|}{\left|w_{C \max }\right|}\right) \frac{\Delta \mathbf{H}\left(z_{s z a}\right)}{\left\|z_{s z a}\right\|}+\sum_{j=1}^{k}\left[\left(\sum_{i=1}^{m\left(z_{j z(j+1)}\right)} \tau_{i}\left(z_{j z(j+1)}\right)\right) \frac{\Delta \mathbf{H}\left(z_{j z(j+1)}\right)}{\left\|z_{j z(j+1)}\right\|}\right] \\
& +\left(\tau\left(z_{s z a}\right)+\frac{\left\|z_{b z g}\right\|}{\left|w_{C \max }\right|}\right) \frac{\Delta \mathbf{H}\left(z_{b z g}\right)}{\left\|z_{s z a}\right\|}+\sum_{j=1}^{k}\left[\left(\sum_{i=1}^{m\left(z_{j z(j+1)}\right)} \frac{\left\|z_{j z(j+1)}\right\|}{\left|w_{C \max }\right|}\right) \frac{\Delta \mathbf{H}\left(z_{j z(j+1)}\right)}{\left\|z_{j z(j+1)}\right\|}\right]
\end{aligned}
$$

where $m\left(z_{a z b}\right) \geq 1$ is the number of times layer $z_{a z b}$ is traversed and $t_{i}\left(z_{a z b}\right)$ the time spent traversing the layer in its $i$ th visit. 
Rearranging to move the minimum time terms to the left side, as well as the first and last intervals where the float first leaves its starting depth and then arrives at the goal depth, the equation becomes

$$
\begin{aligned}
\mathbf{h}(\Gamma)- & {\left[\left(\tau\left(z_{s z a}\right)+\frac{\left\|z_{s z a}\right\|}{\left|w_{C \max }\right|}\right) \frac{\Delta \mathbf{H}\left(z_{s z a}\right)}{\left\|z_{s z a}\right\|}+\left(\tau\left(z_{b z g}\right)+\frac{\left\|z_{b z g}\right\|}{\left|w_{C \max }\right|}\right) \frac{\Delta \mathbf{H}\left(z_{b z g}\right)}{\left\|z_{s z a}\right\|}\right.} \\
+ & \left.\sum_{j=1}^{k}\left[\left(\sum_{i=1}^{m\left(z_{j z(j+1)}\right)} \frac{\left\|z_{j z(j+1)}\right\|}{\left|w_{C \max }\right|}\right) \frac{\Delta \mathbf{H}\left(z_{j z(j+1)}\right)}{\left\|z_{j z(j+1)}\right\|}\right]\right] \\
& =\sum_{j=1}^{k}\left[\left(\sum_{i=1}^{m\left(z_{j z(j+1)}\right)} \tau_{i}\left(z_{j z(j+1)}\right)\right) \frac{\Delta \mathbf{H}\left(z_{j z(j+1)}\right)}{\left\|z_{j z(j+1)}\right\|}\right]
\end{aligned}
$$

where

$$
\left(\sum_{i=1}^{m(d)} \tau_{i}(d)\right) \in[0,+\infty)
$$

The LHS of the equation is simply a $2 \mathrm{D}$ vector and the RHS is a positive but otherwise unconstrained linear combination of the layer horizontal displacements. By the definition of a positively spanning set a valid set of times spent in each layer and hence trajectory can be found that results in this horizontal displacement. The float can thus move any horizontal displacement to any depth, establishing 3D controllability. This completes the proof.

\subsubsection{A convex optimization problem}

The above theorem and proof show that a float trajectory can be found to any needed 3 D location, $\mathbf{x}_{g} \in$. We now provide a method for generating a trajectory to a target location. In this section, we show that the problem can be specified as a convex quadratic program (QP) and solved numerically employing standard algorithms, such as interior-point methods.

An optimization problem consists of finding a value for an optimization variable $\mathbf{x} \in \mathbb{R}^{n}$ that minimizes among all $\mathbf{x}$ that satisfy a number of constraint functions, $f_{i}(\mathbf{x}): \mathbb{R}^{n} \rightarrow \mathbb{R}, i \geq 1$, the function $f_{0}(\mathbf{x}): \mathbb{R}^{n} \rightarrow \mathbb{R}$, the objective function [28]. The problem is convex if and only if all constraint functions and the objective function are convex, where a scalar function $g: \mathbb{R}^{n} \rightarrow \mathbb{R}$ is convex if

$$
g(\alpha x+\beta y) \leq \alpha g(x)+\beta g(y)
$$

for all $x, y \in \mathbb{R}^{n}$ and $\alpha, \beta \in \mathbb{R}$ with $\alpha+\beta=1, \alpha \geq 0, \beta \geq 0$. 
A quadratic program is a particular type of convex optimization problem, where the problem can be expressed in the form [28]

$$
\min _{\mathbf{x}} \frac{1}{2} \mathbf{x}^{T} H \mathbf{x}+f^{T} \mathbf{x}+r
$$

subject to $A \mathbf{x} \leq b$,

$$
C \mathbf{x}=d
$$

where matrices $H \in \mathbb{S}_{+}^{n}, f \in \mathbb{R}^{n}, A \in \mathbb{R}^{m \times n}, C \in \mathbb{R}^{p \times n}, b \in \mathbb{R}^{m}, d \in \mathbb{R}^{p}$ and $r \in \mathbb{R}$. After the notation in Boyd [28], $\mathbb{R}^{n}$ denotes the set of real $n$-column vectors, $\mathbb{R}^{m \times n}$ the set of real $m \times n$ matrices and $\mathbb{S}_{+}^{n}$ the set of $n \times n$ positive semidefinite matrices. A $n \times n$ matrix is positive semidefinite if and only if it is symmetric and $\mathbf{x}^{T} A \mathbf{x} \geq 0$ for all $\mathbf{x} \in \mathbb{R}^{n}$ [16]. The positive semidefinite condition on $H$ is needed to make the objective function convex. As the constraint functions are clearly individually linear and hence convex, the quadratic program is a convex optimization problem.

Let the form, in terms of the sequence of depth layers visited, of the trajectory to be found be fixed. The node visits each layer once to guarantee that the goal location can be reached. The remaining unknown parts of the trajectory to be found are the velocities and times. The problem of finding a trajectory that reaches the goal is equivalent to finding the actuation that minimizes the float's horizontal displacement at the end of the trajectory from the target, where the solution with minimum displacement, 0 , ends up at the goal location and is known to exist. As the square also strictly increases with the displacement, being non-negative, the objective function can also be the square of the horizontal displacement.

The float's horizontal displacement $g$ from the goal at the end of the trajectory, $\mathbf{x}_{g}$ where its starting location is $\mathbf{x}_{s}$, is a function of the times spent traversing each 
trajectory, (5.14), $\mathbf{t}=\left[\begin{array}{llll}t_{1} & t_{2} & \ldots & t_{l}\end{array}\right]^{T}$ The square of the displacement is given by

$$
\begin{aligned}
g^{2}(\mathbf{t})= & \left\|\mathbf{x}_{g}-\left(\mathbf{x}_{s}+\mathbf{h}(\Gamma)\right)\right\|^{2} \\
= & \left(\left(x_{g}-x_{s}\right)-h_{x}(\Gamma)\right)^{2}+\left(\left(y_{g}-y_{s}\right)-h_{y}(\Gamma)\right)^{2} \\
= & \left(\left(x_{g}-x_{s}\right)-t_{1} \frac{\left(\Delta \mathbf{H}\left(d_{1}\right)_{x}\right)}{\left\|d_{1}\right\|}-t_{2} \frac{\left(\Delta \mathbf{H}\left(d_{2}\right)_{x}\right)}{\left\|d_{2}\right\|}-\ldots-t_{l} \frac{\left(\Delta \mathbf{H}\left(d_{l}\right)_{x}\right)}{\left\|d_{l}\right\|}\right)^{2} \\
& +\left(\left(y_{g}-y_{s}\right)-t_{1} \frac{\left(\Delta \mathbf{H}\left(d_{1}\right)_{y}\right)}{\left\|d_{1}\right\|}-t_{2} \frac{\left(\Delta \mathbf{H}\left(d_{2}\right)_{y}\right)}{\left\|d_{2}\right\|}-\ldots-t_{l} \frac{\left(\Delta \mathbf{H}\left(d_{l}\right)_{y}\right)}{\left\|d_{l}\right\|}\right)^{2} \\
= & \left(x_{g}-x_{s}\right)^{2}+\left(y_{g}-y_{s}\right)^{2}-2 \sum_{i=1}^{l}\left(t_{i}\left(x_{g}-x_{s}\right) \frac{\left(\Delta \mathbf{H}\left(d_{i}\right)_{x}\right)}{\left\|d_{i}\right\|}+t_{i}\left(y_{g}-y_{s}\right) \frac{\left(\Delta \mathbf{H}\left(d_{i}\right)_{y}\right)}{\left\|d_{i}\right\|}\right) \\
& +\sum_{i=1}^{l} \sum_{j=1}^{l} t_{i} t_{j}\left(\frac{\left(\Delta \mathbf{H}\left(d_{i}\right)_{x}\right)}{\left\|d_{i}\right\|} \frac{\left(\Delta \mathbf{H}\left(d_{j}\right)_{x}\right)}{\left\|d_{j}\right\|}+\frac{\left(\Delta \mathbf{H}\left(d_{i}\right)_{y}\right)}{\left\|d_{i}\right\|} \frac{\left(\Delta \mathbf{H}\left(d_{j}\right)_{y}\right)}{\left\|d_{j}\right\|}\right) \\
= & \frac{1}{2} \mathbf{t}^{T} H \mathbf{t}+f^{T} \mathbf{t}+r
\end{aligned}
$$

where $r=\left(x_{g}-x_{s}\right)^{2}+\left(y_{g}-y_{s}\right)^{2}, f \in \mathbb{R}^{l}$ and $H \in \mathbb{R}^{l \times l}$. The individual entries in $f$ and $H$ are given by

$$
f_{i}=-2\left(\left(x_{g}-x_{s}\right) \frac{\left(\Delta \mathbf{H}\left(d_{i}\right)_{x}\right)}{\left\|d_{i}\right\|}+\left(y_{g}-y_{s}\right) \frac{\left(\Delta \mathbf{H}\left(d_{i}\right)_{y}\right)}{\left\|d_{i}\right\|}\right)
$$

and

$$
H_{i j}=H_{j i}=2\left(\frac{\left(\Delta \mathbf{H}\left(d_{i}\right)_{x}\right)}{\left\|d_{i}\right\|} \frac{\left(\Delta \mathbf{H}\left(d_{j}\right)_{x}\right)}{\left\|d_{j}\right\|}+\frac{\left(\Delta \mathbf{H}\left(d_{i}\right)_{y}\right)}{\left\|d_{i}\right\|} \frac{\left(\Delta \mathbf{H}\left(d_{j}\right)_{y}\right)}{\left\|d_{j}\right\|}\right)
$$

The times must be greater than the minimum durations needed to traverse each layer at maximum profiling speed

$$
t_{i} \geq \frac{\left\|d_{i}\right\|}{\left|w_{C \max }\right|}
$$

A minimum and maximum total time constraint for the trajectory can also be specified, $\sum_{i=1}^{l} t_{i} \geq t_{m \text { tot }}$ and $\sum_{i=1}^{l} t_{i} \leq t_{M \text { tot }}$.

The constraints on the optimization variable can be written in matrix form

$$
A \in \mathbb{R}^{(l+2) \times l}, b \in \mathbb{R}^{l+2}
$$

where $A_{(l+1) j}=1, b_{l+1}=t_{m \text { tot }}$ and $A_{(l+2) j}=-1, b_{l+2}=-t_{M \text { tot }}$, and for For $i \in[1, l]$,

$$
\begin{array}{r}
A_{i j}=\left\{\begin{array}{cc}
1 & : j=i \\
0 & : j \neq i
\end{array}\right. \\
b_{i}=\frac{\left\|d_{i}\right\|}{\left|w_{C \max }\right|}
\end{array}
$$


The problem can thus be expressed as

$$
\begin{aligned}
& \min _{\mathbf{t}} g^{2}(\mathbf{t})=\frac{1}{2} \mathbf{t}^{T} H \mathbf{t}+f^{T} \mathbf{t}+r \\
& \text { subject to } A \mathbf{t} \leq b
\end{aligned}
$$

where the times are translated into the actuated speed the float needs to move at during the corresponding time period by $\left|w_{C i}\right|=\frac{\left\|d_{i}\right\|}{t_{i}}$.

This is a convex quadratic program if it can be shown that $H$ is positive semidefinite. It is noted that $\frac{1}{2} \mathbf{t}^{T} H \mathbf{t}=\left(h_{x}(\Gamma)\right)^{2}+\left(h_{y}(\Gamma)\right)^{2}$, the square of the horizontal distance of the end of the trajectory from the starting point that cannot be negative, so $\mathbf{t}^{T} H \mathbf{t} \geq 0$. As $H$ is symmetric as well, $H$ is positive semidefinite.

The problem of finding the trajectory of a float to any goal position has thus been expressed as a convex optimization problem. The primary advantage of doing so is that convex optimization problems are easily solvable, with it being said that if a practical problem can be formulated as a convex optimization problem it is solved [28]. Convex optimization problems have been studied extensively in mathematics, due to problems of this form appearing in numerous application areas from signal processing to statistics and finance, and convex problems having the property that any locally optimal point is also globally optimal. As a result, highly efficient algorithms exist for solving such problems. Interior-point methods can find the optimal solution to arbitrary precision in polynomial time [106] and using these, personal computers can solve problems with hundreds of variables and thousands of constraints in a few seconds [28].

Given the small footprint of the solver, the algorithm can be implemented on each float, which have limited computational capabilities, and each device can rapidly individually solve the convex optimization problem to find trajectories to any location, provided that initial information to generate the matrix values are supplied. For this problem, a measurement of the current profile, the node's current position, the goal location and the format of the trajectory are needed.

\subsection{Trajectory generation}

Each node solves a convex optimization problem of the form in (5.33) to find its trajectory. The problem is stated for a generic trajectory (5.14). The specific trajectory form solved in our trajectory generator algorithm is now described. To ensure that each layer is visited, the float descends to the bottom layer before 
returning to the surface. This movement being described as a cycle. Two issues are raised.

- A feasible trajectory may be found that only approaches near the lattice point, at height $z_{g}$ ( $g$ being also the index of the depth in the height discretization), during the descent phase of the cycle rather than the ascent and vice versa.

- The anticipated position of the float after cycling to the surface should also put it in good stead to close with the lattice point the next time a trajectory is computed.

To resolve the second point raised, the convex optimization problem of Equation 5.33 is modified to find not only the first cycle but a subsequent cycle as well, terminating at the height of the lattice point in the ascent phase of the second cycle. The problem seeks to minimize the sum of the squared distance $g^{2}\left(c_{1}\right)$ the float is able to come within the lattice point, calculated when the device is at the height of the target $z_{g}$, during its first cycle and the discounted distance $g^{2}\left(c_{2}\right)$ it is able to reach in the second cycle. The objective function is

$$
g^{2}\left(c_{1}\right)+a_{c 2} g^{2}\left(c_{2}\right)
$$

where the discount factor, $0 \leq a_{c 2} \leq 1$. The discount factor causes less emphasis to be placed on the approach in the second cycle as the current may not actually be non-time-varying nor non-horizontally spatially varying. It is less certain that the second cycle will be accurately followed, but indicates that the surfacing point is likely to eventuate in a future cycle ending up near the target point. The sequence of layers visited in the trajectory is

$$
\begin{array}{r}
\left\{z_{1 z 2(1)}, z_{2 z 3(2)}, \ldots, z_{(g-1) z g(g-1)}, \ldots, z_{k z(k+1)(k)}, z_{(k+1) z k(k+1)}, \ldots, z_{(g+1) z g(2 k+1-g)}, \ldots\right. \\
\left.z_{2 z 1(2 k)}, z_{1 z 2(2 k+1)}, \ldots, z_{(g-1) z g(2 k+g-1)}, \ldots, z_{k z(k+1)(3 k)}, z_{(k+1) z k(3 k+1)}, \ldots, z_{(g+1) z g(4 k+1-g)}\right\}
\end{array}
$$

In brackets in the subscript is the index of each layer in the list.

To account for the first issue, four convex optimization problems of the form below are solved to generate the trajectory:

$$
\min _{\mathbf{t}} \frac{1}{2} \mathbf{t}^{T} H \mathbf{t}+f^{T} \mathbf{t}+r
$$

subject to $A \mathbf{t} \leq b$, 
where the objective function

$$
\begin{aligned}
& \frac{1}{2} \mathbf{t}^{T} H \mathbf{t}+f^{T} \mathbf{t}+r \\
& =\mathcal{T}_{11} g^{2}\left(\mathbf{t}_{g-1}\right)+\mathcal{T}_{21} g^{2}\left(\mathbf{t}_{2 k+1-g}\right)+a_{c 2} \mathcal{T}_{31} g^{2}\left(\mathbf{t}_{2 k+g-1}\right)+a_{c 2} \mathcal{T}_{41} g^{2}\left(\mathbf{t}_{4 k+1-g}\right)
\end{aligned}
$$

The expression $g^{2}\left(t_{a}\right)$ is the squared horizontal displacement of the float, at the end of time interval $a$, from the lattice point as given by (5.27). The entries inside the matrices are thus

$$
\begin{aligned}
& a_{1}=\mathcal{T}_{11}+\mathcal{T}_{21}+a_{c 2} \mathcal{T}_{31}+a_{c 2} \mathcal{T}_{41} \\
& a_{1 i}= \begin{cases}1 & : i<=g-1 \\
0 & : \text { otherwise }\end{cases} \\
& a_{2 i}= \begin{cases}1 & : i<=2 k+1-g \\
0 & : \text { otherwise }\end{cases} \\
& a_{3 i}= \begin{cases}1 & : i<=2 k+g-1 \\
0 & : \text { otherwise }\end{cases} \\
& a_{4 i}= \begin{cases}1 & : i<=4 k+1-g \\
0 & : \text { otherwise }\end{cases} \\
& r=a_{1}\left(x_{g}-x_{s}\right)^{2}+\left(y_{g}-y_{s}\right)^{2} \\
& f_{i}=-2 a_{1}\left(a_{1 i}+a_{2 i}+a_{3 i}+a_{4 i}\right)\left(\left(x_{g}-x_{s}\right) \frac{\left(\Delta \mathbf{H}\left(d_{i}\right)_{x}\right)}{\left\|d_{i}\right\|}+\left(y_{g}-y_{s}\right) \frac{\left(\Delta \mathbf{H}\left(d_{i}\right)_{y}\right)}{\left\|d_{i}\right\|}\right) \\
& H_{i j}=H_{j i}=2 a_{1}\left(a_{1 i}+a_{2 i}+a_{3 i}+a_{4 i}\right)\left(\frac{\left(\Delta \mathbf{H}\left(d_{i}\right)_{x}\right)}{\left\|d_{i}\right\|} \frac{\left(\Delta \mathbf{H}\left(d_{j}\right)_{x}\right)}{\left\|d_{j}\right\|}+\frac{\left(\Delta \mathbf{H}\left(d_{i}\right)_{y}\right)}{\left\|d_{i}\right\|} \frac{\left(\Delta \mathbf{H}\left(d_{j}\right)_{y}\right)}{\left\|d_{j}\right\|}\right)
\end{aligned}
$$

The matrix $H$ is positive semidefinite as it is the sum of positive semidefinite matrices, having been established in Section 5.3.1 that the $H$ matrices of the individual squared horizontal displacements are positive semidefinite. 
The problem is solved for $T=\left[\begin{array}{llll}1 & 0 & 0 & 1\end{array}\right]^{T},\left[\begin{array}{llll}1 & 0 & 1 & 0\end{array}\right]^{T},\left[\begin{array}{llll}0 & 1 & 0 & 1\end{array}\right]^{T}$, $\left[\begin{array}{llll}0 & 1 & 1 & 0\end{array}\right]^{T}$. In this form, trajectories are found that minimize the combined displacement from the lattice position on the descent and ascent phases of the first and second cycles, descent and descent phases, ascent and ascent phases and ascent and descent phases respectively. The solution with the least objective function value is then selected by the node, which the float then follows until the end of the first cycle. A new trajectory is then calculated.

The time constraints are specified by

$$
\begin{gathered}
A_{i j}=\left\{\begin{array}{cc}
1 & : j=i \\
0 & : j \neq i
\end{array}\right. \\
b_{i}=\frac{\left\|d_{i}\right\|}{\left|w_{C \max }\right|}
\end{gathered}
$$

for $i \in[1,4 k+1-g]$.

$$
\begin{aligned}
& A_{(4 k+2-g) j}=\left\{\begin{array}{ll}
1 & : j \leq 2 k \\
0 & : j>2 k
\end{array},\right. \\
& b_{4 k+2-g}=t_{m \text { tot }} \\
& A_{(4 k+3-g) j}=\left\{\begin{array}{ll}
-1 & : j \leq 2 k \\
0 & : j>2 k
\end{array},\right. \\
& b_{4 k+3-g}=-t_{M \text { tot }} \\
& A_{(4 k+4-g) j}=\left\{\begin{array}{ll}
1: & 2 k+1 \leq j \\
0: & j \leq 2 k
\end{array},\right. \\
& b_{4 k+4-g}=t_{m \text { tot }} \\
& A_{(4 k+5-g) j}=\left\{\begin{array}{ll}
-1: & 2 k+1 \leq j \\
0: & j \leq 2 k
\end{array},\right. \\
& b_{4 k+5-g}=-t_{M \text { tot }}
\end{aligned}
$$

The latter limits impose minimum and maximum constraints on the total length of time the first and second cycles can take. A maximum time limit of 10 hours is set 
for each cycle in the simulation scenario and no (or a 0 ) minimum time limit is set. This is deemed to give enough scope for the float to vary its profiling velocity to find a linear combination of layer times that brings the device close to the lattice point. At the same time it is short enough so that nodes frequently pass by the lattice point, in order that an unmanageable number of floats is not needed before the random distribution of their arrivals means that a node is present and covering the region around the point at each time instant. With a maximum actuated speed of $0.2148 \mathrm{~ms}^{-1}$, the least amount of time that a float can complete one cycle between heights $0 \mathrm{~m}$ and $-500 \mathrm{~m}$ in the scenario is 1.29 hours.

\subsubsection{Practical considerations}

The convex optimization problems requires information on the current profile and the node's location. In this section, we describe how these obtained. Rather than acquiring the current velocity along the water column from numerical ocean model current predictions, which have high uncertainties [179] as discussed in Section 5.1.5, each node is equipped with an acoustic doppler current profiler (ADCP) and uses this device to measure the current profile.

Underwater vehicles are able to measure the instantaneous current velocity above and below them using an Acoustic Doppler Current Profiler (ADCP) instrument, also known as a Doppler Velocity Log (DVL) when employed to track the velocity of the vehicle. The ADCP does this by emitting pulses of sound vertically. As the sound travels, it is reflected by particles, such as sediment, suspended at different layers. These particles drift at the velocity of the local current and so the reflected signal is also frequency shifted due to the Doppler effect [122]. This allows the instrument to reconstruct the velocity of the current across the depth profile that the node is located at from the frequency of reflected signals and using the time when the signal was received and the propagation speed of sound to determine the depth.

ADCPs embody a practical measurement tool for profiling floats and are already standard equipment for other unmanned underwater vehicles, such as AUVs and gliders [104, 132].

The sound pulses emitted by the device do not interfere with network acoustic communications as the frequencies employed by the ADCP are typically much higher, around $300 \mathrm{kHz}$ [183]. Due to the frequency-dependent attenuation of sound underwater, which increases with frequency, the usable bandwidth for acoustic communications is limited to around $0-100 \mathrm{kHz}$ for distances less than $100 \mathrm{~m}$ and $0-10 \mathrm{kHz}$ over $1-10 \mathrm{~km}$ [187]. As seen in Chapter 4 where this modem was employed 


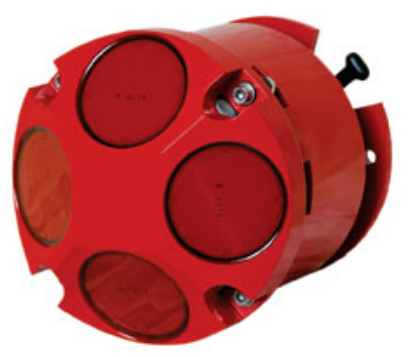

(a)

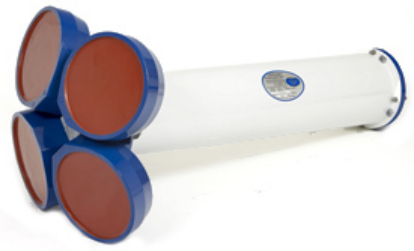

(b)

Figure 5.6: Various DVLs and ADCPs produced by Teledyne RD Instruments [5]. These are mounted on AUVs and buoys to measure the vehicle's velocity relative to the ground and the current velocity below the sensor.

for the simulation scenario after other works also chose this device [113,205], the operating frequency of the LinkQuest UWM1000 underwater acoustic modem is between $26.77-44.62 \mathrm{kHz}$.

The sound pulses emitted by the ADCP are likewise attenuated, limiting the distance at which a reflected signal can be perceived. The maximum distance at which the device can measure the current velocity depends on the signal frequency being employed. For instance, the Workhorse Navigator DVL produced by Teledyne RD Instruments operating at a frequency of $300 \mathrm{kHz}$ has a range of $200 \mathrm{~m}$, whereas the Workhorse Long Ranger ADCP is able to measure currents up to $600 \mathrm{~m}$ away with a $75 \mathrm{kHz}$ signal [5]. This $600 \mathrm{~m}$ range is sufficient to evaluate the current along the entire depth profile in typical scenarios. A $500 \mathrm{~m}$ deep network deployment is fairly standard [205]. In this case, the float may have to perform one pass each with the ADCP rotated upwards then downwards.

In order to compute current velocities, the velocity of the float must be determined to factor it out of observations [122]. This is done by measuring the frequency shift of a signal reflected from a layer with known, stationary speed, the seabed. The ADCP is said to have acquired bottom lock if it is able to receive a reflection from the seafloor. This may not always be the case in deep water for our scenario. However, as current velocity tends to decrease with increasing depth, the deepest water layer from which a reflection is obtained can be regarded as a level of no motion and the float velocity determined from there [183]. In terms of accuracy, the Workhorse Long Ranger ADCP, which has a range of $600 \mathrm{~m}$ and uses the $75 \mathrm{kHz}$ band, is described in its datasheet as having an accuracy of $\pm 1 \%, \pm 5 \mathrm{mms}^{-1}[5]$.

Concerns are the energy consumption and cost of ADCPs. The Workhorse 
Navigator DVL uses $66 \mathrm{~W}$ of power while transmitting [5]. However, in our strategy the ADCP is only turned on briefly to establish the current profile at the start of each float's cycle. The time for the float to then move is at minimum $1.29 \mathrm{~h}$, the time needed to traverse up and down the height of the network $(500+500 \mathrm{~m})$ at the maximum float speed $0.2148 \mathrm{~ms}^{-1}$, so the energy expended in ADCP measurements can be disregarded. A more significant issue is the ballooning in price if each float is to be equipped with an ADCP. The cost of a typical ADCP is around $\$ 12,000-19,000$, resulting in a doubling or trebling of the unit cost of a float and mitigating the chief, economic advantage that floats have over AUVs.

As for how the node determines its position, localization can be challenging underwater $[58,190]$. While on the surface, a device can use GPS to acquire its coordinates but the RF GPS signals do not penetrate underwater. For underwater localization, acoustic signals with the device's location and transmission time are sent by nodes. Floats determine their distance to nodes whose signals they receive by dividing the difference between when the message was transmitted and received by the propagation speed, and triangulate their position (they know their depth). Only anchor nodes on the surface with access to GPS initially know where they are located and can broadcast messages. As other, underwater nodes are able to evaluate their positions, they can begin transmitting, iteratively localizing the network. Since nodes drift with currents, the process must be continually updated. Requiring underwater localization adds a communications overhead to an already severely constrained channel and assumes connectivity, in that the node must be within acoustic communications range of three other floats that can be localized themselves.

In profiling along the entire depth profile, the float must surface, so it makes sense that the trajectory through its assigned lattice position be computed from where the node surfaces and resurfaces, since the process is repeated. The node is able to then evaluate its starting position by GPS and underwater localization can be dispensed with.

\subsubsection{Outline}

The steps carried out by our trajectory generation algorithm, run locally on each float, are summarized here.

1. The float has been assigned a lattice position to frequent, $x_{g}$.

2. The depth range over which the float profiles is divided into $k+1$ heights, where $z_{1}$ and $z_{k+1}$ are the greatest (surface) and lowest heights respectively. Included 
in this sequence is the height of the goal location, $z_{g}, g$ also denoting the index of the height in the list. This gives rise to $k$ layers, $\left\{z_{1 z 2}, z_{2 z 3}, \ldots, z_{k z(k+1)}\right\}$.

3. The float is on the surface.

4. Using GPS the device acquires its position, $\mathbf{x}_{s}$. It also probes the water with its ADCP to measure the current velocity along the depth profile at its location, $\mathbf{v}_{U}\left(x_{s}, y_{s}, z, t_{s}\right)$. From this it computes the value of

$$
\frac{\Delta \mathbf{H}\left(z_{a z b}\right)}{\left\|z_{a z b}\right\|}
$$

for each layer by numerical integration of Equation 5.15 .

5. Substituting the values it has measured, the float computes a cycle trajectory minimizing its horizontal displacement to the goal location when it passes through its depth according to Equation 5.36. If currents and float kinematic constraints permit, the device will cross the target.

6. The float executes the first cycle in the generated trajectory, ending up on the surface after completion.

7. Steps 3 to 6 are repeated, generating the float's movements up until the end of the mission.

\subsection{Target assignment}

In this section, we detail how target assignment is carried out in our scheme.

Nodes are linked to the lattice points they are to cover according to a one-time minimax assignment, performed at the start of the mission. In this strategy, the aim is to find a target lattice point for each node so that the maximum horizontal displacement a float is from its target is minimized. This, rather than say a random assignment, is done to increase the likelihood that the current profile, which is only ideally non-horizontally spatially varying, measured at the float's starting location also applies to the goal position, only a short distance away, and to the path found in between. This enhances the ability of the scheme to find accurate trajectories through the targets.

The minimax assignment algorithm proposed by Kone et al. [103] is employed to find the linkages. First, $n$ degenerate lattice positions are constructed. This is done by ordering the $m$ unique lattice locations into a sequence, then going through it 
in ascending and revolving order, adding each position to an initially empty target set until the set consists of $n$ positions. If $n$ is a multiple of $m$, this results in an equal number of copies of each unique lattice position ending up in the set of target locations. The goal of the algorithm is to find a one-to-one association between each node and each point in the target set.

A distance-based minimax assignment is iteratively found by first computing a minisum target assignment that finds an allocation that minimizes the total horizontal displacement of each float from its target lattice point. In our implementation of Kone et al.'s [103] scheme, a community developed MATLAB implementation [31] of Munkres' algorithm [3] for solving minisum assignment problems in polynomial time is utilized. The algorithm requires the positions of each node and lattice point and is processed at a single location.

While still at this central location, the individual minisum node assignment with the longest horizontal displacement is removed from the set of all possible node to lattice assignments. The minisum assignment is then recalculated for this modified graph. If the maximum cost link in this solution is less than in the previous minisum assignment, the process is repeated, successively stripping links from those that are considered. If the maximum cost link is equal or greater than the previous assignment, the past solution is minimax.

Execution of the algorithm on our workstation takes under a second.

This is a centralized algorithm. Centralization is not considered an issue as, since nodes are deployed by air or ship, all floats will initially be located on the surface when the assignment is carried out. Each node can determine its position by GPS and communicate and retrieve their assignment using $\mathrm{RF}$. As radio communications is high-bandwidth and with all floats being within one-hop range of each other, no difficulties are expected in being able to co-ordinate this.

In the simulation scenario, nodes begin at uniformly distributed 3D positions so that the results of this scheme can be compared to others, such as providing no control to the floats. The floats are considered as being partway through their trajectories, which are then generated assuming that in a real network they were obtained at some prior time on the surface. The lattice points are assigned as targets to the nodes based on their horizontal displacement from their starting positions, distributed in 3D space. Initial trajectories for the nodes are computed using an amended form of (5.36), where the cost of the second cycle is only to be minimized, the float already following the first cycle. It is randomly decided whether the float is on the descent or ascent portion of the first cycle, so the node has a $50 \%$ chance of 
beginning the scenario moving up or down.

\subsection{Limitations}

As the scheme relies on trajectories generated by solving a quadratic program of the form of (5.33) to guide floats to lattice positions, it is limited to the same environments for which the program was formulated. Namely, where the current is non-time-varying, non-horizontally spatially varying and the current along the depth profile positively spans the 2D plane. Only then is the node's displacement described exactly by the equations in the convex optimization problem. The path followed by the float is approximately described by the program for slowly varying currents, but as further variation is introduced accuracy deteriorates and results in unintended float behaviour.

The scheme does not guarantee full coverage at every time instant. Coverage performance will vary between trials, even if the initial positions of the floats and the current are unchanged, depending on the internal state of the float's processing environment and the random duration trajectories produced by the solver algorithm. Performance is thus unpredictable.

\subsection{Summary}

In this chapter, we presented our node movement scheme that co-ordinates the movement of nodes in a float-based UWSN to extend the network coverage. Our solution is based on assigning floats to move to lattice points that fully cover the RoI, with the eventual goal of spacing the arrival times of floats at each lattice point so they can be continuously covered. The scheme is validated in simulation in the subsequent chapter. 


\section{Chapter 6}

\section{Performance of a coverage extension node movement scheme}

This chapter details the simulations we use to evaluate the performance of Scheme 1, our novel finite-velocity profiling float coverage extension movement scheme, which we proposed in Chapter 5.

The realistic depth-decaying coastal current mobility, Current 2, that we developed earlier on in in this thesis (Table 3.6) is chosen to define the flow in our simulations. The scheme is not assessed on its ability to cover a static $\operatorname{RoI} \mathcal{R} \subseteq \mathbb{R}^{3}$, as might be supposed based on (5.6). Scheme 1 is developed for well-behaved currents, which floats can exploit to be 3D controllable. This does not apply to Current 2, where the flow lies in one direction (eastwards). Our scheme, and not only our scheme but all float motion planning strategies, will not be able to prevent all profilers from eventually moving eastwards out of the RoI. For an analysis of the 3D controllability of Current 2, refer to Appendix B.

It should be emphasized that this does not mean that Scheme 1 is unlikely to work for static monitoring in all currents. As shown by $[130,180]$, large parts of various ocean regions exhibit controllability properties that the scheme relies upon. The Humboldt current is another real-world example. Flowing off the coast of Chile, within $500 \mathrm{~m}$ of the surface the current positively spans the plane in 2D and with inevitably any horizontal variation 3D, where the current flows predominantly northwards between $0-100 \mathrm{~m}$ depths, southwards between $100-400 \mathrm{~m}$ and northwards from $400-500 \mathrm{~m}$ depths [174].

Instead, we evaluate the float movement scheme in another important sensing application: Lagrangian tracking [43]. Not all underwater features are stationary. Pollution features in particular, such as harmful algal blooms and patches of low- 
oxygen water corresponding to chemical spills, drift with the surrounding water. The evolution of these phenomena is best studied with a sensor network that moves with the feature.

Our simulations are based around a float-based UWSN being tasked with covering a fixed-size drifting RoI that is moving at the velocity of the current at its centre point. Define a body-fixed frame attached to the RoI at its drift point, the centre of the RoI where the velocity of the surrounding current gives the velocity of the RoI, as shown in Figure 6.1. The orthogonal coordinate system of the body-fixed frame is chosen with the $x$-axis made to point along the drift parcel's instantaneous direction of motion, the surge direction [116] (resulting in $z$ being vertically upward when the current is horizontal, as in Current 2). The RoI rotates with the drift parcel and so is distributed over fixed body-fixed frame coordinates. Scheme 1 is directly extensible to this application, as the rate of change of a float's body-fixed coordinates is the sum of a relative current velocity field term, $\mathbf{v}_{r U}(t, \stackrel{B}{\mathbf{x}})$, and the profiling velocity of the float in both the static and drifting frames, $\mathbf{v}_{C}(t)$ :

$$
\stackrel{B}{\mathbf{v}}(t)=\mathbf{v}_{C}(t)+\mathbf{v}_{r U}(t, \stackrel{B}{\mathbf{x}})
$$

where

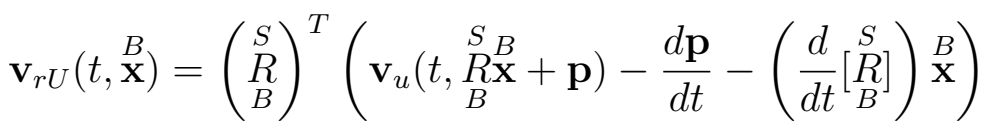

and

$$
\mathbf{v}_{C}(t)=\left[\begin{array}{lll}
0 & 0 & w_{C}(t)
\end{array}\right]^{T}
$$

See Section B.3 for the derivation of this equation. The problem of utilizing float mobility $\mathbf{v}_{C}(t)$ in a current $\mathbf{v}_{U}(t, \mathbf{x})$ to cover a rigid body drifting RoI can then be considered to be the same as using float mobility $\mathbf{v}_{C}(t)$ to cover a rigid body RoI, Scheme 1's designed application (see (5.6)), where the current is now given by $\mathbf{v}_{r U}(t, \stackrel{B}{\mathbf{X}})$.

Scheme 1 can then be simply modified to cover the drifting RoI by replacing world frame node and lattice positions with body-fixed frame positions, and replacing the world frame current velocity with the relative current velocity. To allow each float to obtain its relative position and the relative current velocity along the depth profile at its location, we assume that the drift point, the location of the origin $\mathbf{p}$, is tagged by a drogued drifter $[130,176]$. Such a device consists of a parachute lowered to the depth of the feature and which is connected by a tethered to a buoy floating on 


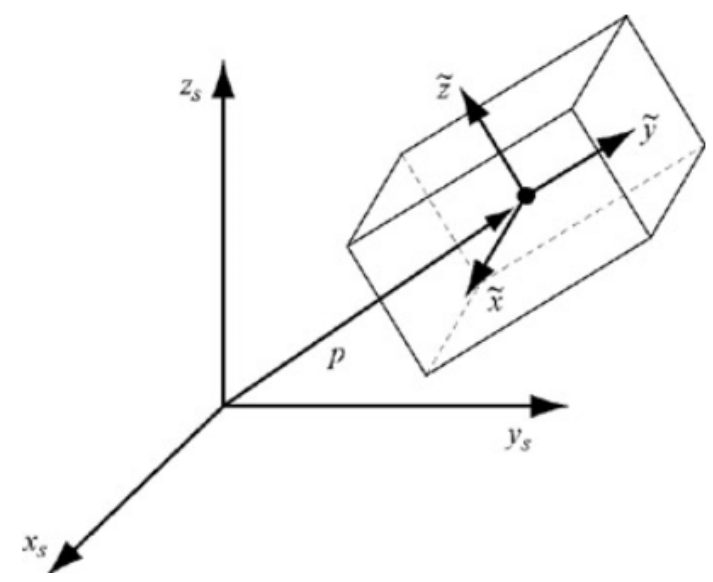

Figure 6.1: The body-fixed frame: attached to the rigid body (box) RoI is a body-fixed frame $x-y-z$, with origin $\mathbf{p}$ at the location of the drift point. Shown also is the stationary world frame $x_{s}-y_{s}-z_{s}$. Image from Choset et al [39].

the surface. The drag induced in the parachute causes the instrument to drift with the current velocity at the depth of the parachute. The buoy contains an electronics package with a GPS receiver for determining its position. The drifter calculates its position and derived velocity from GPS, and communicates this across the surface to floats when they are ready to generate a trajectory. Two transmissions are sent, one for these quantities at time $t-\Delta t$ and another for these quantities at time $t$. As the tether is unlikely to be straight, it is assumed that multiple inclinometers and gyrocompasses are placed along it so the curve traced by the tether can be determined and the position of the drogue and not just the buoy can be found.

The value of $\mathbf{p}(t-\Delta t), \mathbf{p}(t)$ and $\mathbf{v}_{U}(\mathbf{p}, t-\Delta t), \mathbf{v}_{U}(\mathbf{p}, t)$ are then combined with the position of the float found using GPS and the inertial current velocity recorded by the float using its $\mathrm{ADCP}, \mathbf{v}_{U}(t, \stackrel{S}{\mathbf{x}})$, to derive all other needed variables to find the relative positions (B.7) and current velocity (6.2):

$$
\begin{aligned}
\frac{d \mathbf{p}}{d t} & \approx \frac{\mathbf{p}(t)-\mathbf{p}(t-\Delta t)}{\Delta t} \\
\stackrel{S}{R} & =\left[\begin{array}{ccc}
\frac{u_{u}(\mathbf{p}, t)}{\left\|\mathbf{v}_{u}(\mathbf{p}, t)\right\|} & \frac{v_{u}(\mathbf{p}, t)}{\left\|\mathbf{v}_{u}(\mathbf{p}, t)\right\|} & 0 \\
\frac{-v_{u}(\mathbf{p}, t)}{\left\|\mathbf{v}_{u}(\mathbf{p}, t)\right\|} & \frac{u_{u}(\mathbf{p}, t)}{\left\|\mathbf{v}_{u}(\mathbf{p}, t)\right\|} & 0 \\
0 & 0 & 1
\end{array}\right] \\
\frac{d}{d t}\left[{ }_{B}^{S}(t)\right] & \approx\left[\begin{array}{ccc}
\left.\frac{\left[u_{u}(\mathbf{p}, t)\right]-\left[u_{u}(\mathbf{p}, t) \|\right.}{\left\|\frac{u_{u}(\mathbf{p}, t-\Delta t)}{\| v}(\mathbf{p}, t-\Delta t)\right\|}\right] \\
\Delta t & \ldots & 0 \\
\ldots & \ldots & 0 \\
0 & 0 & 0
\end{array}\right]
\end{aligned}
$$

In our simulations, a time interval of $\Delta t=1 \mathrm{~s}$ is used. 
Unlike the static RoI scenario, features of Current 2 can be exploited by float mobility to preserve coverage of a drifting RoI. In this current, the velocity of the flow is in the local downstream direction of the jet and decreases in magnitude with increasing depth. As seen in Figure 4.5, nodes drifting on the surface are carried further along eastwards than those remaining closer to the bottom. In the drifting frame of reference, the lower, slower current is seen as going backwards, as evidenced in Figure B.40. The figure is a top-down plot of the relative current velocity field at time $t=0 \mathrm{~s}$ for a drifting RoI centred at $\left[\begin{array}{lll}3750 & 0 & -250\end{array}\right]^{T}$ in the middle of the jet. At each grid point is drawn the overlaid normalized relative current velocity at 21 heights spaced $25 \mathrm{~m}$ apart from $0 \mathrm{~m}$ to $-500 \mathrm{~m}$. The co-ordinates in the figure are the body-fixed frame coordinates. Along each depth profile, there are relative currents pointing in the positive and negative $x$-directions. Scheme 1 finds node trajectories to return them to their original lattice positions with minimum displacement. Each float repeatedly computes a cycle trajectory that has the profiler move to the bottom then top of the RoI, then back down. The use of a convex optimization solver guarantees for this cyclic type of trajectory, the minimum displacement trajectory is found. The current conditions indicate that, in the Lagrangian tracking application, a cycle can totally eliminate the displacement in one horizontal axis with a variable displacement in the other horizontal axis. This is achieved by removing the positive horizontal contribution while staying in the faster, upper layers with the time spent in the deeper, backwards-directed layers. The minimum displacement trajectory found by the convex optimization problem solver must have a displacement of this one-axis displacement elimination trajectory or less. Hence, in this scheme, nodes are able to at least return to the same horizontal co-ordinate and close to their original position in the RoI to provide coverage at later times.

Performance is assessed in our simulations by the scheme's deployment cost and coverage, and the distance travelled and energy consumed by floats. The results demonstrate that our scheme provides coverage more cost-effectively than utilizing AUVs only to track the RoI. Unlike a straightforward dive and rise scheme or having the floats perform no profiling, which result in zero area coverage after two days, our scheme is able to provide an ongoing level of coverage of the region to be monitored.

This contents of this chapter are as follows. Section 6.1 describes the simulation setup. The different schemes that are evaluated are detailed in Section 6.2. Section 6.3 defines our performance metrics and how they are computed. Our results are presented in Section 6.4, and Section 6.5 summarizes the chapter. 


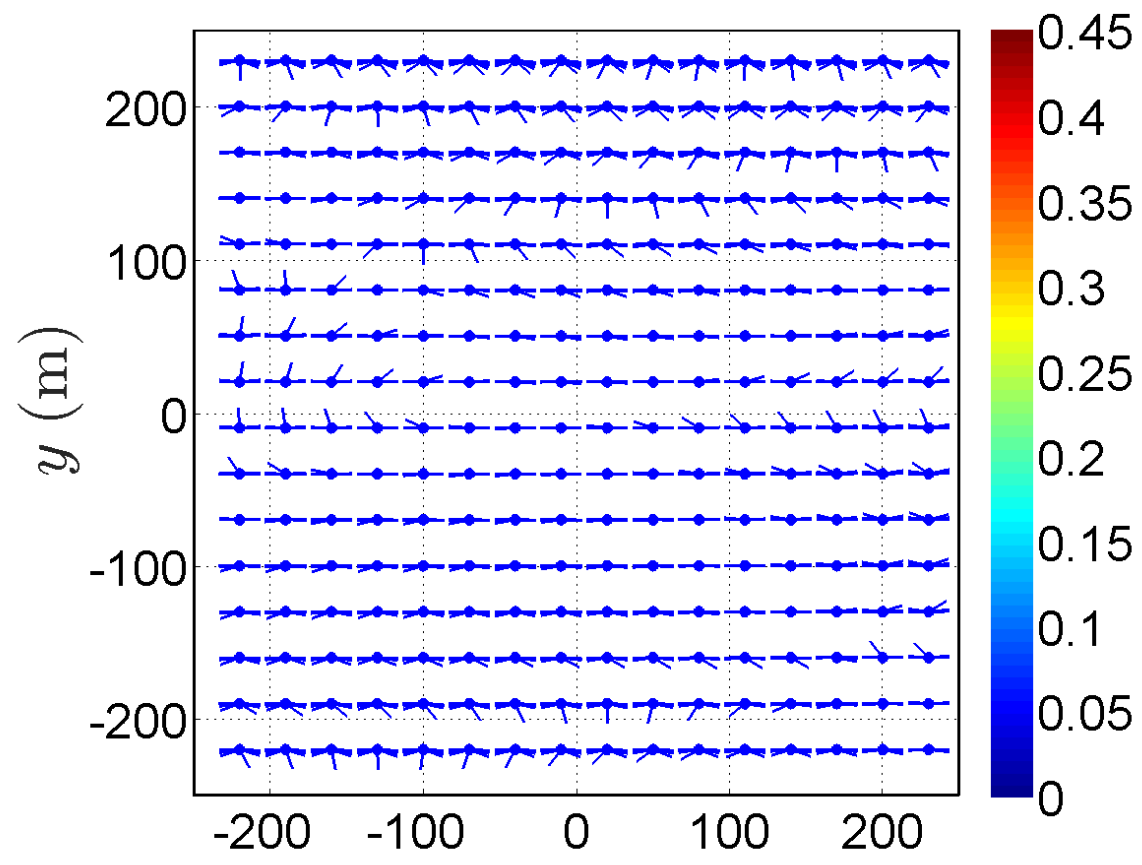

Figure 6.2: Top-down view of the relative current velocity field for a drifting RoI located in the centre of Current 2 at time $t=0 \mathrm{~s}$.

\subsection{Simulation settings}

Our simulations are carried out using a custom discrete-event network mobility simulator that we coded in MATLAB for this study. As a discrete-event simulator, the simulator maintains a queue of events that are scheduled to execute at particular simulation times. Events are dynamically added to the queue during the course of the simulation. The events are executed in sequential time order by the simulator. During the execution of an event, the network state is updated based on the nature of the scheduled event, and upon completion the simulator moves to the next event in time. In this way, the simulator advances discretely rather than continuously in time from one event to the next [159]. The network state (specifically: the body-fixed frame's orientation and each node's position and velocity, both in world-frame co-ordinates) is output to file every $120 \mathrm{~s}$, giving a record of the simulation. Appendix $\mathrm{C}$ gives further, technical information on the simulator's code architecture.

The current velocity in the simulation is given by Current 2 (3.17). The node's mobility is computed by numerically integrating the velocity function to find the node's position over time, in common with other works that study the motion of bodies in water $[37,61]$. In our case, the differential equation solver we use 
is a stiff solver based on a modified Rosenbrck formula of order 2 [126]. A stiff solver is needed as the node's actuated velocity specified by Scheme 1 is given by a series of periods of constant velocity connected by instantaneous velocity transitions (mathematically: Heaviside steps, which are stiff). Standard fourth-order RungeKutta particle-following algorithms, which are designed to solve nonstiff problems, are unsuitable in this situation as they require the step size to be taken extremely small to compute an accurate solution approximation [139].

An interior-point algorithm (MATLAB's quadprog [8]) is utilized to solve the convex optimization problem in Scheme 1's trajectory generator routine (Section 5.4). Runtime of the function takes no more than a few seconds on a desktop computer with an Intel i5-3470 (Quad-Core, 3.20 GHz) processor and 8 GB of RAM running 64-bit Linux and MATLAB. Computation issues in implementing a solver on a less capable actual float are therefore expected to be non-existent. An observation with respect to the solver is that it tends to produce trajectories either the length of the minimum or maximum total time constraint. In the minimax target assignment nodes close to a lattice point are granted it as their target. If also the floats pass the lattice point at the same time, the nodes will tend to arrive around the same time rather than one after another so that there is continual coverage. In order to spread out the arrival times, the total time constraints could be randomly varied in the convex optimization problem solved by each float, but this was not explored.

Consider a deepwater blowout event, in the mold of the Deepwater Horizon incident that has occurred in a coastal area. The pollution that resulted from this disaster was detailed in Section 2.4, and resulted in the formation of an underwater oil plume. In our simulations, a network of $n=30-80$ floats, with number depending on the specific trial, is deployed at time $t=0 \mathrm{~h}$ over a 5 day mission to sense the underwater oil plume that has formed. In the 2010 oil spill, plumes were detected between $5-500 \mathrm{~m}$ depth [29], so the task is to cover a $500 \mathrm{~m}$ diameter, $500 \mathrm{~m}$ high cylindrical RoI having a vertical axis, extending from the surface to $500 \mathrm{~m}$ below it, centred about a drifting point in the middle of the plume at $250 \mathrm{~m}$. The centre is initially located in the centre of the current jet at $\left[\begin{array}{lll}3750 & 0 & -250\end{array}\right]^{T}$. A cylinder is chosen as the actual physical location it occupies in the world frame remains invariant when it is rotated in the body-fixed frame. A full list of the simulation settings is to be found in Table 6.1.

The density profile in the area, $\rho_{\mathrm{W}}(z)$, is given by Figure 6.3 and reflects conditions in the Gulf Stream off Cape Hatteras, the same area where the MCM model parameters were established [26]. The density profile is based on hydrographic mea- 
Table 6.1: Simulator settings

\begin{tabular}{|c|c|c|}
\hline Parameter & Symbol & Value \\
\hline Mission duration (days) & & 5 \\
\hline Number of nodes & $n$ & $30-80$ \\
\hline Sensing range $(\mathrm{m})$ & $R_{S}$ & 150 \\
\hline Communications range $(\mathrm{m})$ & $R_{C}$ & 150 \\
\hline Seawater density $\left(\mathrm{kgm}^{-3}\right)$ & $\rho_{\mathrm{W}}(z)$ & As in Figure 6.3 \\
\hline Pressure at height (dbar) & $p(z)$ & $10.1-1.01 \mathrm{z}$ \\
\hline Sea level pressure (dbar) & $p_{0}$ & 10.1 \\
\hline Gravity of Earth $\left(\mathrm{ms}^{-2}\right)$ & $g$ & 9.81 \\
\hline Sampling interval (s) & $\Delta T$ & 120 \\
\hline Unit cost (float) & & $\$ 9,000$ \\
\hline Unit cost (AUV) & & $\$ 250,000$ \\
\hline \multicolumn{3}{|l|}{ ALACE float parameters } \\
\hline Velocity limits $\left(\mathrm{ms}^{-1}\right)$ & {$\left[w_{C D \max }, w_{C U \max }\right]$} & {$[-0.2202,0.2001]$} \\
\hline Battery capacity (MJ) & $E_{\text {cap }}$ & 1 \\
\hline Mass, ballasted $^{1}(\mathrm{~kg})$ & $\mathrm{m}$ & 25.4 \\
\hline Drag area $\left(\mathrm{m}^{2}\right)$ & $A$ & 0.0962 \\
\hline Drag coefficient & $C_{\mathrm{D}}$ & 1.4 \\
\hline Volume, fixed, in air $\left(\mathrm{m}^{3}\right)$ & $V_{\mathrm{H}_{0}}$ & 0.0243 \\
\hline Compressibility $\left(\mathrm{dbar}^{-1}\right)$ & $\gamma_{H}$ & $2.20 \times 10^{-6}$ \\
\hline Active buoyancy element volume $\left(\mathrm{m}^{3}\right)$ & $V_{\mathrm{B}}$ & {$[0,7.50] \times 10^{-4}$} \\
\hline
\end{tabular}

surements of the depth, temperature and salinity in the Gulf Stream, which we have converted to density. For details of this computation, refer to Section E.2. The data is obtained from the World Ocean Database 2013 (WOD13) [6], an online-accessible freely available collection of records of casts taken from around the world. The database is maintained by the USA's National Oceanic and Atmospheric Administration (NOAA) as part of its Ocean Data Archive, and its use here is supported by its employment as a resource in other underwater acoustic network simulators [70].

Being air dropped at the target, the floats begin uniformly randomly distributed within the cylinder. For each profiler, uniformly distributed random numbers $h, r$ and $\theta$ in the intervals $[-500,0] \mathrm{m},[0,1] \mathrm{m}$ and $[0,2 \pi]$ respectively are generated.The node's starting height in the simulation is $h$ and its $x$ - and $y$-coordinates in the 


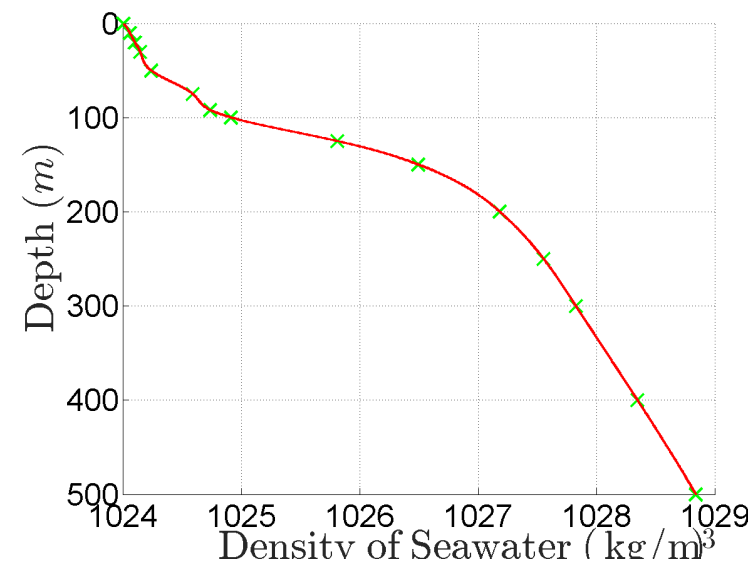

Figure 6.3: Density profile of the simulation area

relative motion frame are

$$
\begin{gathered}
x=250 \sqrt{r} \cos \theta, \\
y=250 \sqrt{r} \sin \theta
\end{gathered}
$$

This gives the node a probability of being placed into any region within the cylinder equal to the ratio of the measure of this area to that of the RoI. It would have been incorrect to place floats so that

$$
\begin{aligned}
& x=250 r \cos \theta, \\
& y=250 r \sin \theta
\end{aligned}
$$

as this results in more nodes being distributed closer to the centre of the crosssection [201]. The random number generator is seeded with a different value in each trial, so the initial co-ordinates of the nodes varies between runs.

Each float has a sensing and communications range of $150 \mathrm{~m}$. The rationale for utilizing this sensing range is explained in Section 6.2. The float parameters are based on the ALACE Autonomous Lagrangian Circulation Explorer float [47]. These floats have been globally deployed as part of the Argo Program [192], a worldwide initiative to monitor the subsurface ocean using an un-network array of around 3000 profiling floats. The ALACE float is chosen as it is one of the few profilers for which comprehensive specifications are available. Furthermore, our results should have greater relevance to the many scholars who are familiar with these types of floats from being involved in the Argo Program. The float parameters are taken from the ALACE design document [47]: the compressibility of the ALACE float is known to be $\gamma_{H}=2.20 \times 10^{-6} \mathrm{db}^{-1}$ and its drag coefficient is $C_{D}=1.4$. The area 
presented to the fluid while the float is profiling up and down is that of its circular damping disk, of radius $0.175 \mathrm{~m}$, so its effective drag area is $C_{D} A=0.1347 \mathrm{~m}^{2}$, where $A=\pi r^{2}=0.0962 \mathrm{~m}^{2}$. The shape of the float body is approximated as being a $1.07 \mathrm{~m}, 0.085 \mathrm{~m}$ radius long cylinder, giving a volume of $\pi r^{2} h=0.0243 \mathrm{~m}^{3}$. The ALACE float can be equipped with a 20-cell alkaline pack that can provide $10^{6} \mathrm{~J}$ of electrical energy before discharging. The use of actual float parameters leads to more representative results of what our scheme can achieve in practice.

For simplicity, the node movement schemes are restricted to only allow floats to ascend between $0-500 \mathrm{~m}$ depth, the extent of the RoI. The maximum upwards and downwards profiling velocities that the floats can move at all points within these depth bounds are given by:

$$
\left[w_{C D \max }, w_{C U \max }\right]=\left[w_{C D}\left(z_{\min }\right), w_{C U}\left(z_{\max }\right)\right]
$$

where

$$
w_{C D}\left(z_{\min }\right)=-\sqrt{\frac{2 g}{C_{\mathrm{D}} A}} \sqrt{\frac{m}{\rho_{\mathrm{W}}\left(z_{\min }\right)}-\left(V_{\mathrm{Bmin}}+V_{\mathrm{H}_{0}}\left[1+\gamma_{H}\left(p\left(z_{\min }\right)-p_{0}\right)\right]\right)}
$$

and

$$
w_{C U \max }=\sqrt{\frac{2 g}{C_{\mathrm{D}} A}} \sqrt{\left(V_{\mathrm{Bmax}}+V_{\mathrm{H}_{0}}\left[1+\gamma_{H}\left(p\left(z_{\max }\right)-p_{0}\right)\right]\right)-\frac{m}{\rho_{\mathrm{W}}\left(z_{\max }\right)}}
$$

Section D.2 explains how this expression is derived and the terms used. In the scenario, floats are set to be neutrally buoyant midway down the RoI, at $250 \mathrm{~m}$ depth, with their bladder inflated to half-capacity, so $V_{B}=375, \mathrm{~cm}^{3}$. Solving for $m$ in (D.16),

$$
\begin{array}{r}
w_{C}(-250)=0, \\
\left(V_{\mathrm{B}}+V_{\mathrm{H}_{0}}\left[1+\gamma_{H}\left(p(-250)-p_{0}\right)\right]\right)-\frac{m}{\rho_{\mathrm{W}}(-250)}=0, \\
m=\rho_{\mathrm{W}}(-250)\left(0.000375+V_{\mathrm{H}_{0}}\left[1+\gamma_{H}\left(p(-250)-p_{0}\right)\right]\right)
\end{array}
$$

the float's mass needs to be $m=25.4 \mathrm{~kg}$. This simply means that the profiler needs to be ballasted with an additional $2.4 \mathrm{~kg}$ on top of its ordinary mass of $23 \mathrm{~kg}$ [47].

Substituting these values into (6.7), the maximum downward and upward speeds that the float can move throughout $z=[-500,0] \mathrm{m}$ are

$$
\begin{aligned}
& w_{C U \max }=w_{C U}(0)=0.2001 \mathrm{~ms}^{-1} \\
& w_{C D \max }=w_{C D}(-500)=-0.2202 \mathrm{~ms}^{-1}
\end{aligned}
$$




\subsection{Evaluated schemes}

Four node movement schemes are evaluated in our study:

\section{No control}

Providing a baseline level of performance, the network is simulated with the nodes performing no profiling and simply passively drifting with the currents.

\section{Dive and rise (DNR) scheme}

In the DNR scheme, each float repeatedly cycles between the minimum and maximum depth bounds of the RoI at a constant profiling speed of $0.2 \mathrm{~ms}^{-1}$. In the simulation, each node is initialized with a random direction to begin moving along, with an equal probability of ascending or descending. The float is equipped with a pressure sensor. On detecting that it has hit or passed depth $0 \mathrm{~m}$ or $500 \mathrm{~m}$ it reverses direction until it strikes the opposing boundary, $500 \mathrm{~m}$ or $0 \mathrm{~m}$ depth respectively, and the process is repeated until the end of the mission.

DNR is compared against our Scheme 1, as this algorithm presents a simple method for improving coverage in our scenario. Initially, all nodes are randomly deployed inside the RoI, providing a high level of coverage that probabilistically increases as the number of nodes rises. Instead of drifting only with the currents, a goal of mobility may be to return nodes to the same positions. While not guaranteeing full coverage, the initial formation demonstrated high coverage and the network attempts to reform it. As seen in Figures 3.15, the current velocities in Current 2 vary approximately linearly with depth. Near the centre of the RoI, the velocity is zero at mid-depth, $250 \mathrm{~m}$, the depth of the tagged particle, since this feature is stationary in the Lagrangian frame. Above this point the velocity increases linearly, and below this point it decreases linearly. Thus, a float executing a complete cycle of the RoI will spend an equal time and be carried an equal distance forwards and backwards, returning to its original position.

This scheme does not require any communications, each float's behaviour being able to be preprogrammed, computation or special equipment, aside from an inexpensive pressure sensor [205] that is standard-issue among floats. Compared to this simple strategy, Scheme 1 should show significantly improved coverage performance to justify the cost of fitting each float with an ADCP. 


\section{Scheme 1}

Scheme 1, in its moving RoI tracking mode of operation, is implemented. In the convex optimization problem (5.36) that is solved to produce the profiler controls, the maximum speed that can be specified is $w_{C \max }=0.2 \mathrm{~ms}^{-1}$, at the limit of what can be physically achieved by the float. The trajectory is discretized by layers, $\mathcal{Z}=\{0,-25,-50, \ldots,-450,-475,-500\} \mathrm{m}$. The projected displacement of the second cycle from the target, which is not actually executed, is prioritized less in the objective function, with a discount factor of $a_{c 2}=0.75$.

A minimum and maximum constraint on the total duration for each cycle are imposed of $t_{m \text { tot }}=3 \mathrm{~h}$ and $t_{M \text { tot }}=10 \mathrm{~h}$. The minimum time constraint is a carryover from a simulated annealing-based scheme proposed by the authors. In that scheme, on surfacing, a central ASV would compute velocity controls for the float for a trajectory lasting till its next surfacing. In order to give the ASV enough time to generate a trajectory, a minimum duration constraint is imposed to limit the frequency of surfacings. The central controller can count on an average time between a float from throughout the network ending up on the surface, and a request for a trajectory being made. The presentation of the results for Scheme 1 were intended to be compared to this strategy, which was considered more promising, and so the same parameters were used where possible. The performance of the simulated annealing-based strategy was found to be not significantly improved over that of Scheme 1 while adding a centralized dependence to the scheme requirements. Consequently, the scheme is omitted from this chapter as well as for reasons of space. No minimum time constraint is needed for Scheme 1. The simulations for Scheme 1 had already been done however with $t_{m \text { tot }}=3 \mathrm{~h}$, and insufficient time was left to redo them. The results are likely to underestimate the performance Scheme 1 could achieve with no minimum time constraint, since over a shorter time the actual trajectory traced by a float will tend to deviate less from the convex optimization problem assumed trajectory due to the current being time-varying. At maximum profiling speed, the float may complete a cycle in $1.39 \mathrm{~h}$.

Regarding the target lattice, an effort has been made to find an arrangement that fully covers the RoI while using the minimum number of nodes. Assuming a binary disk sensing model, the regular placement of nodes that fully covers an unbounded space while using the least number of devices is well known in 2D. This is achieved by placing nodes at the centres of regular hexagons or, equivalently, at the vertices of a grid of equilateral triangles, where the radii of each hexagon or the length of the sides of each triangle is equal to $R_{S}$ or $\sqrt[3]{R_{S}}$ respectively. $R_{S}$ is the sensing range of 
each node. The asymptotic optimality of this solution is rigorously proven in a 1939 paper [98].

Recently, with the research community having been made aware of the advantages that UWSNs could bring [11], there has been interest in finding the placement of sensors that covers a 3D space using the minimal number of nodes. Unlike 2D networks, no regular tessellation of nodes has been proven to be optimal for the 3D case.

Alam and Haas [13] propose placing nodes at the centres of the cells of a regular uncurved truncated octahedron tessellation, which is equivalent to positioning a node at each vertex of a body-centred cubic lattice. This guarantees full coverage and the authors suggest that it may also require the least number of nodes. The minimal node number coverage problem is restated in terms of Voronoi cells. The Voronoi cell of a node is the set of all points in space that are closer to the node than any other sensor in the network. Let the RoI be all of $\mathbb{R}^{3}$. In a fully-covered network, the size of each Voronoi cell is bounded by the sensing range. The set of all Voronoi cells tessellate the whole space. Restricting the solution to a regular Voronoi tessellation, the network can be thought of as requiring a node to be deployed per volume $V_{\text {vor }}$ of the RoI, where $V_{v o r}$ is the constant volume of the Voronoi cell. The density of nodes throughout a float arrangement with smaller $V_{\text {vor } 1}$ will be greater by a factor of $V_{\text {vor } 2} / V_{\text {vor } 1}$ when compared to a deployment with Voronoi cell volume $V_{\text {vor } 2}$. The coverage problem thus becomes that of finding the space-filling Voronoi cell shape with maximum volume. Assuming sphere-based sensing, the Voronoi cell must also lie within the node's circumsphere of radius $R_{S}$, so the problem consists of finding the space-filling shape with the highest ratio of its volume to that of its circumsphere, termed volumetric quotient by the authors.

The authors hypothesise that the cell that has the highest volumetric quotient also minimizes the surface area. This is the case with a sphere, which has the maximum ratio of 1 and also the highest ratio of volume of surface area. According to Kelvin's conjecture that was formulated in 1887, the optimal way to fill a 3D space with cells of equal volume so that the surface area is minimized, is via tessellation of a 14-sided truncated octahedron having a very slight curvature of the hexagonal faces [194]. Correspondingly, Alam and Haas develop a lattice pattern placing nodes at the centres of the cells of a truncated octahedron tessellation. The volumetric quotient is found to be 0.68329 , higher than other regular arrangements such as a cubic tessellation. This confirms that it will use fewer nodes and lending credence that it may be optimal. 
In the paper [13], a method for constructing a truncated octahedron lattice is given. From a starting point $\left[\begin{array}{lll}x & y & z\end{array}\right]^{T}$, place nodes at (the requirement for the formation to also provide connectivity is ignored here, so $R$ from the original equations is $R_{S}$ ):

$$
\left[x+(2 u+w) \frac{2 R_{S}}{\sqrt{5}} \quad y+(2 v+w) \frac{2 R_{S}}{\sqrt{5}} \quad z+w \frac{2 R_{S}}{\sqrt{5}}\right]^{T}
$$

where $u, v, w \in Z$. For Scheme 1, this equation is employed to produce a formation that fully covers the RoI, where $\left[\begin{array}{lll}x & y & z\end{array}\right]^{T}$ is chosen to be the centre of the cylinder. In body-fixed frame co-ordinates this is $\left[\begin{array}{lll}0 & 0 & -250\end{array}\right]^{T}$. The lattice node locations are then used as the target positions $l_{i}$.

A problem with (6.12) that it does not give a stopping condition for when the RoI is fully covered. To get only the nodes needed to cover the cylinder, the output of the equation for all $u, v, w$ satisfying the constraints

$$
\begin{aligned}
& -250-5 R_{S} \leq(2 u+w) \frac{2 R_{S}}{\sqrt{5}} \leq 250+5 R_{S} \\
& -250-5 R_{S} \leq(2 v+w) \frac{2 R_{S}}{\sqrt{5}} \leq 250+5 R_{S} \\
& -500-5 R_{S} \leq w \frac{2 R_{S}}{\sqrt{5}} \leq 0+5 R_{S}
\end{aligned}
$$

is obtained. This ensures that all lattice points within the cylinder plus an additional five sensing range lengths around the RoI are generated, so that when the Voronoi diagram of the nodes over $\mathbb{R}^{3}$ is produced, the Voronoi cells of all nodes that can sense part of the cylinder are constrained by neighbouring nodes to within a sphere $R_{S}$ around it. The Voronoi cells are checked for intersection with the RoI, approximated as a polyhedron, and only those that do so are retained. For computing the Voronoi diagram and the intersection of polyhedra, functions from MPT [2], a computational geometry toolbox, are used.

The number of nodes needed to cover the RoI depends on the sensing range. The lattice was generated for different sensing ranges, the number of nodes in each being plotted in Figure 6.4. For this simulation, the maximum number of nodes is limited to 80 so that each run could complete in reasonable time. This resulted in $R_{S}=150 \mathrm{~m}$ being selected. With 80 floats, this is roughly 3 times as many as the minimum needed to cover the RoI. This margin was deemed sufficient to begin comparing the advantages and disadvantages of employing a large number of cheaper but less capable floats compared to a smaller number, equal to that of the lattice nodes, of more expensive but omnidirectional AUVs, which can remain fully covering 
the RoI by remaining on the lattice points. The sensing range is consistent with UWSN transmission ranges in other simulations [205].

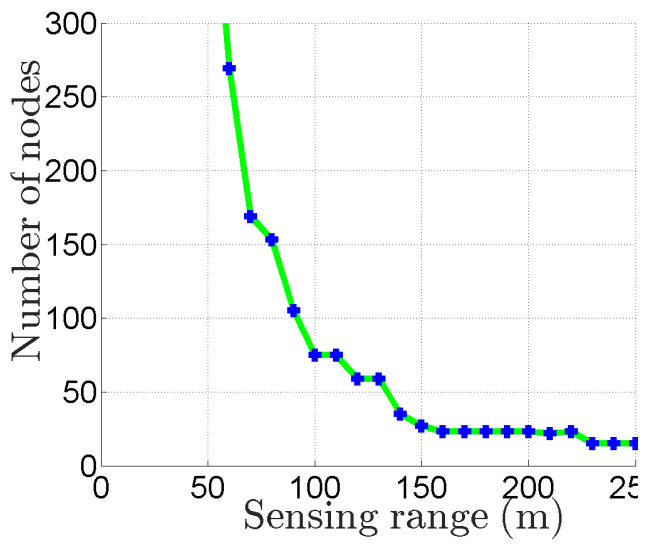

Figure 6.4: Minimum number of nodes needed to cover the cylindrical RoI for various sensing ranges.

The generated points call for some nodes to be placed above the surface, which is impossible in practice. The heights of these points are set to $z=0 \mathrm{~m}$. However, now the formation may have more nodes than needed for full coverage. The Voronoi diagram of the remaining points is recalculated and nodes successively removed in ascending order of the volume of their cell until the coverage falls below 1 . The last sensor is then replaced. Figure 6.5 shows the lattice, consisting of 23 target points produced for a sensing range of $150 \mathrm{~m}$. These are used for the target points in Scheme 1, where the co-ordinates in the body-fixed frame of the targets are listed in Table 6.2 for repeatability of the results.

\section{AUV deployment}

The coverage problem has been complicated in this thesis by the use of floats. Being only able to move up and down under their own control, floats are dependent on the currents as to where they end up. In certain currents, full coverage over time may be impossible to meet and Scheme 1 does not guarantee full coverage. The use of floats is motivated by being much cheaper than AUVs. However, AUVs can achieve full coverage via a simple and obvious behaviour.

In this scheme, also referred to as the back-to-original positioning [121] scheme, it is assumed that AUVs are able to pilot themselves to remain at a fixed position in the RoI. At the start of the simulation, an AUV is located at each of the positions given by Table 6.2. The total number of vehicles, 27 , is the minimum number of 


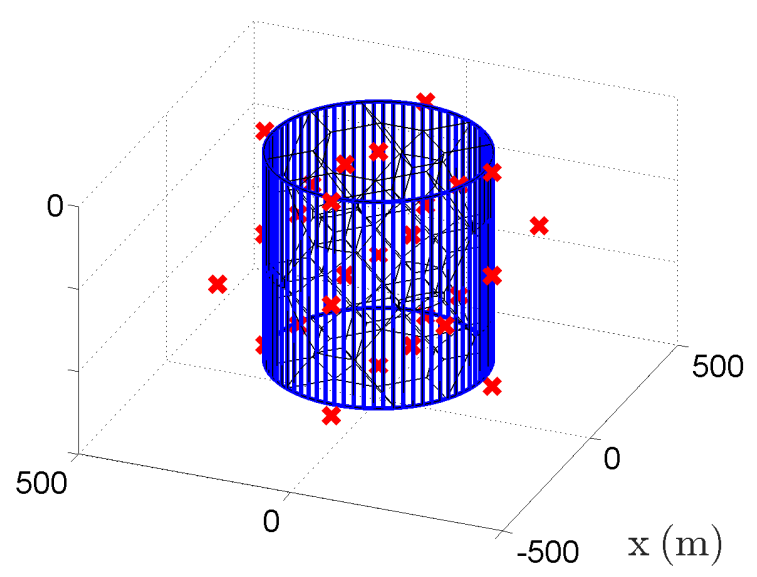

Figure 6.5: Positions, marked red, of targets/AUVs (in Scheme 1/AUV deployment scheme) in the scenario and the Voronoi diagram associated with this deployment. Axes are the body-fixed frame axes.

AUVs that is capable of keeping the RoI fully covered. At each time instant, each AUV, being omnidirectional, adjusts its actuated velocity in the body-fixed frame to be equal and opposite to the relative current velocity at its position, resulting in its co-ordinates in the relative motion frame being unchanged. The maximum velocities of AUVs is around $1.5-2.1 \mathrm{~ms}^{-1}[54,104]$, so it can always match the current speed. The relative current speed throughout the simulation area is around $0.1 \mathrm{~ms}^{-1}$, one order lower, as seen in Figure 6.2. Beginning from a formation that fully covers the RoI, the AUVs remain in this formation. For a float network to be justified, a similar level of coverage performance must be achieved at much reduced cost compared to the AUV solution.

\subsection{Metrics}

This section presents the metrics used to analyse the performance of the node movement schemes in simulation and how they are evaluated from the simulation record. Previous works employing floats have only looked at their scheme's primary objective, either the level of coverage or aggregation $[42,130]$ or in trajectory planning problems the distance from the goal [182]. The RoI coverage is also evaluated here. However, a secondary objective, energy is also likely to influence which scheme is actually selected for implementation. A strategy with slightly reduced coverage performance but significantly lower energy consumption rates may be preferable to another scheme, especially if nodes in the other scheme run out of energy before they can complete the mission. 
Table 6.2: Target/AUV (in Scheme 1/AUV deployment scheme) positions given as co-ordinates in and fixed in the body-fixed frame

\begin{tabular}{|c|c|c|c|}
\hline Node & $x(\mathrm{~m})$ & $y(\mathrm{~m})$ & $z(\mathrm{~m})$ \\
\hline 1 & 0 & 0 & -250 \\
\hline 2 & 134.2 & -134.2 & -384.2 \\
\hline 3 & 134.2 & 134.2 & -384.2 \\
\hline 4 & -134.2 & 134.2 & -384.2 \\
\hline 5 & -134.2 & -134.2 & -384.2 \\
\hline 6 & -134.2 & 134.2 & -115.8 \\
\hline 7 & 134.2 & -134.2 & -115.8 \\
\hline 8 & 134.2 & 134.2 & -115.8 \\
\hline 9 & -134.2 & -134.2 & -115.8 \\
\hline 10 & 0 & 0 & 0 \\
\hline 11 & 0 & 0 & -518.3 \\
\hline 12 & 268.3 & 0 & -250 \\
\hline 13 & 0 & 268.3 & -250 \\
\hline 14 & -268.3 & 0 & -250 \\
\hline 15 & 0 & -268.3 & -250 \\
\hline 16 & 0 & 268.3 & 0 \\
\hline 17 & 268.3 & 0 & 0 \\
\hline 18 & 0 & -268.3 & 0 \\
\hline 19 & -268.3 & 0 & 0 \\
\hline 20 & 0 & 268.3 & -518.3 \\
\hline 21 & 268.3 & 0 & -518.3 \\
\hline 22 & 0 & -268.3 & -518.3 \\
\hline 23 & -268.3 & 0 & -518.3 \\
\hline
\end{tabular}

The coverage performance establishes what level of success to expect of the scheme in a real-world deployment. What is not established is whether a float UWSN should be used in the first place. As seen with the back-to-original positioning scheme, with the ability to move in any direction, AUVs can in a simple manner maintain coverage of the entire region at all times. The concession for full actuation is that AUVs are generally much more expensive to produce than floats. To approach the same level of coverage many more less capable floats may have to be deployed, but by being also multiple times cheaper, it may be more cost-efficient to employ a float network. 
This idea is investigated by producing cost-coverage tradeoff charts of AUV and float solutions.

The analysis of the simulation differs from other works by considering the energy consumption of the nodes and the economic rationale for the schemes.

\subsubsection{Deployment cost}

The monetary cost for assembling the network is regarded as the sum of the unit costs of each node,

$$
n_{A U V} c_{A U V}+n_{\text {float }} c_{\text {float }}
$$

where $n_{A U V}$ and $n_{\text {float }}$ are the number of deployed AUVs and floats, and in this scenario the price of an AUV is $c_{A U V}=\$ 250,000$ [143] and for a float $c_{\text {float }}=$ $\$ 9000[178]$.

\subsubsection{Coverage}

The positions of each node is known, so calculating the area coverage should be a simple matter of finding the measure of the union of each float's sensing spheres and its intersection with the cylindrical RoI. In fact, this is not a straightforward task. Whereas the volume of two spheres can be calculated by considering all the cases where the two objects intersect, there are many ways for multiple spheres to intersect with each other and the RoI.

Yet knowing the volume of the union of multiple spheres is important in nuclear physics and molecular biology, influencing how proteins and DNA molecules interact, and it has been evaluated in a number of works for these topics [17,102]. Avis et al. [17] present an exact algorithm for computing this volume. The algorithm constructs the Laguerre-Voronoi diagram of the space, such that the space is partitioned into polygonal cells, with one cell for each sphere. The volume of the union can then be found by summing the volume of the intersection of each sphere and its corresponding cell. This approach was not used in this thesis, as it was considered too time-consuming to code and ensure that the algorithm was correctly implemented according to its description in the paper. ProGeom [102] is a freely downloadable Fortran program for computing different aspects of the geometry of protein molecules. This includes evaluating the volume of the union of spheres. ProGeom was trialled by the author, but was found to give apparently correct values at times for the volume of the union of the sensing spheres of the nodes in the network and clearly 
incorrect values at other times. This, along with the program providing a fixed text output and requiring a fixed text input, which made it difficult to interface the Fortran program with the Matlab simulator led to its rejection. On reflection now, the incorrect values may have resulted when the regions covered by the nodes was partitioned, which ProGeom's algorithm, being designed for a single contiguous protein molecule, may not have accounted for.

Even if either method had been adopted, it would have been insufficient for computing the area coverage. The area coverage of the network is the volume of the union of the sensing spheres of the nodes and its intersection with the RoI. No algorithm for directly computing this value could be found in the literature.

Instead, a numerical, Monte Carlo method [90] is employed to evaluate the coverage at a recorded time from the saved node positions and location of the RoI. $N$ points are uniformly randomly distributed within the cylindrical RoI (6.5). The distance of each point from each node is computed, and the point flagged if a point is within $R_{S}$ of it. The coverage proportion is taken to be the ratio of flagged points to the total number of points. As $N$ increases to infinity, this value converges to the true coverage proportion. A value of $N=10,000,000$ is employed in the simulation, which is assumed to result in negligible uncertainty between the estimated and actual coverage proportion. Efficiency is not an issue due to the computation being done offline.

\subsubsection{Cumulative coverage}

The instantaneous coverage is not the goal specifically of the schemes. In Section 5.2, an objective function (5.10) was defined for the strategies as the cumulative or 3D coverage proportion over time, which is evaluated approximately from the coverage proportion at each time by the Trapezoid Rule as

$$
\int_{t_{a}}^{t_{b}} C_{p}(t) d t \approx \frac{\Delta T}{2} \sum_{i=1}^{k}\left(C_{p}\left(t_{a}+(i-1) \Delta T\right)+C_{p}\left(t_{a}+i \Delta T\right)\right)
$$

In the graphs, most often the normalized cumulative coverage will be plotted, where this value is divided by the timespan and can be interpreted as the average coverage proportion over the interval. This provides a one value evaluation of the relative success of a network deployment. 


\subsubsection{Float energy consumption}

In other works, the lifetime of mobile nodes in UWSNs has been measured by the distance travelled under its own power by the device. Nodes are considered to have run out of energy once they have moved a fixed total distance [121]. This distance does not accurately reflect the actual lifetime of a float. For example, to travel downwards the float does not need to use any energy. The work needed to move fluid out of the external bladder, reducing the profiler's buoyancy and causing it to accelerate downwards, is provided by the ocean, at higher pressure than the float's internal reservoir. The energy stored in the float's battery can be utilized to keep the device running at a later date, whereas, as the profiler has descended some distance, the float's lifetime has been considered to be reduced using the distance metric.

In general, the time the node travels a constant total distance can only be equated to the time when it runs out of energy if the rate at which it uses up its electrical energy is related to its actuated speed by

$$
P=k v
$$

where $k$ is a scalar, in which case the energy expended in crossing a distance $d$ at constant speed is the same regardless of the velocity used

$$
E=P t=v \times \frac{k d}{v}=k d
$$

Using the distance metric as such implies that the energy expenditure is related linearly to speed, which is neither the case for floats nor AUVs [184]. Unsatisfied by this distance metric, an energy function for a float was derived in Section D.3.

The float's total energy used in mobility up until the time $t_{b}$ of a record from the start of the simulation, $t_{a}$, is given by

$$
\begin{array}{r}
W=\int_{t=t_{a}}^{t=t_{b}} \mathcal{P}(t) d t \\
=\int_{t=t_{a}}^{t=t_{b}}\left\{\begin{array}{cc}
E(z(t)) \frac{\partial V_{\mathrm{B}}}{\partial t}, & \frac{\partial V_{\mathrm{B}}(t)}{\partial t}>0 \\
0, & \frac{\partial V_{\mathrm{B}}(t)}{\partial t} \leq 0
\end{array}\right\} d t
\end{array}
$$

In practice, we only have access to discrete time points in the simulation record. An estimate of the energy consumed is computed by reconstructing the value of the integrand at all the time samples in between $t_{a}$ and $t_{b}, t_{a}, t_{a}+\Delta T, t_{a}+2 \Delta T, \ldots, t_{a}+$ $(k-1) \Delta T, t_{b}$. Having the node's speed and position, the program can substitute these into the fitted functions for the float's pumping efficiency (D.29) and seawater 
density (Figure 6.3), to find $E(z)$ and the volume of the profiler's active buoyancy element, $V_{\mathrm{B}}$ (D.33), at each time. The derivative of the volume is calculated from the first order forward difference of the volume between successive time samples,

$$
\frac{\partial V_{B}\left(t_{i}\right)}{\partial t} \approx \frac{\widehat{\partial V_{B}(t)}}{\partial t}=\frac{V_{B}\left(t_{i+1}\right)-V_{B}\left(t_{i}\right)}{\Delta T}
$$

resulting in the integrand being approximated as

$$
\mathcal{P}\left(t_{i}\right) \approx \begin{cases}E\left(z\left(t_{i}\right)\right) \frac{\widehat{\partial V_{B}\left(t_{i}\right)}}{\partial t}, & \frac{\widehat{\partial V_{B}\left(t_{i}\right)}}{\partial t}>0 \\ 0, & \frac{\widehat{\partial V_{B}\left(t_{i}\right)}}{\partial t} \leq 0\end{cases}
$$

The energy is then computed using the Trapezoid Rule,

$$
\begin{aligned}
& W=\int_{t=t_{a}}^{t=t_{b}} \mathcal{P}(t) d t \\
& \approx \frac{\Delta T}{2} \sum_{i=1}^{k}\left(\mathcal{P}\left(t_{a}+(i-1) \Delta T\right)+\mathcal{P}\left(t_{a}+i \Delta T\right)\right)
\end{aligned}
$$

\subsubsection{Distance travelled}

As a metric presented in the literature [121] and to compare it with the actual energy consumed, the distance travelled by the nodes using their own mobility is plotted. The distance travelled is given exactly by

$$
d=\int\|\mathbf{v}(t)\| d t
$$

With the velocity at each time sample being able to determined as $\mathbf{v}=\left[\begin{array}{lll}0 & 0 & w_{C}(t)\end{array}\right]^{T}$ for floats, the points can be numerically integrated using the Trapezoid Rule to obtain an approximate value for the distance travelled:

$$
d \approx \frac{\Delta T}{2} \sum_{i=1}^{k}\left(\left\|v\left(t_{a}+(i-1) \Delta T\right)\right\|+\left\|v\left(t_{a}+i \Delta T\right)\right\|\right)
$$

\subsubsection{Hybrid AUV-float UWSNs}

Due to the limited controllability of floats in the scenario, it was acknowledged in Section B.4 that some target locations may not be reachable by floats. This suggests that to cover these portions of the network, an AUV needs to be deployed. With floats providing coverage of other areas, not all 27 AUVs may be required to 
guarantee full coverage. Significant increases in coverage may be gained by deploying a few vehicles alongside the float network. In the simulation, the coverage that can be achieved by inserting 1, 2 or 3 AUVs into the network is evaluated. The number of supplementary AUVs that is needed for the coverage to not fall below $80 \%$ is also evaluated.

Being omnidirectional, it is assumed that AUVs can move to any position at the next sampled time. The maximum coverage that can be achieved after placing an additional $n$ AUVs and the minimum number of AUVs required to exceed the 90\% coverage threshold are estimated using a greedy approach, after Liu et al [119]. At each time record, the world frame axis-aligned $500 \mathrm{~m} \times 500 \mathrm{~m} \times 500 \mathrm{~m}$ cuboid area centred on the drifting feature and enclosing the cylindrical RoI is divided into cubes with sides $5 \mathrm{~m}$ long. A cube is denoted as needing to be covered if its centre lies within the RoI and is not within sensing range of a node. Cycling through all the cubes, an AUV is placed at the cube that decreases the number of cubes to be covered by the most. Treating the just placed AUV as an additional node in the network, the updated coverage is computed via the Monte Carlo method described in Section 6.3.2 and further AUVs are placed with this vehicle contributing to which cubes are covered. For the $75 \%$ threshold estimate, AUVs are continually placed until the proportion is exceeded or 27 AUVs have been deployed, which if arranged in a truncated octahedron lattice would be able to provide full coverage and so further nodes are not needed.

This does underestimate the number of additional nodes required as the finite velocity of the AUVs is not taken into consideration. AUVs would not always be able to instantaneously move to the greedy locations at each timestep.

\subsubsection{Partial AUV Coverage}

At the same time as we are adding AUVs to the float UWSN, we remove vehicles from the AUV lattice to investigate the coverage proportion that can be achieved with less than the minimum number of nodes needed for full coverage. Beginning with an empty lattice, AUVs are greedily added to the formation. A node is temporarily added to an unoccupied lattice position and the Monte Carlo coverage computed, before the AUV is removed from the network. This is repeated for each lattice position in turn. An AUV is then permanently placed at the lattice position that saw the greatest coverage increase, and the process repeated for the lattice consisting of the previously inserted nodes. It is hoped that the measured coverages reflect the maximum values that can be obtained with a fixed number of nodes. The purpose 
of this exercise is to determine the cumulative coverage cost efficiency that can be achieved with AUVs, where the application only requires partial coverage, in order to compare it with a float-based solution.

\subsection{Results}

Each scenario was simulated 10 times with different randomly generated starting node positions. At the time, the intention was to simulate the network in the jet, separatrix and vortex, in order that the scheme behaviours could be observed in all aspects of the current. Another simulated annealing scheme was also to be modeled that ran in real time. This was a feature of the strategy to give the controller the maximum time to perform local searches, finding the best possible solutions, throughout the mission duration, which cannot exceed the simulation time. The number of runs was chosen to allow the planned simulations to be completed within the time that computing resources were allocated, with the simulations executed remotely on a pair of dedicated server machines. The results for the initially jet-centred region are presented. The plotted values are the median across the 10 runs, with the error bars enclosing $60 \%$ of the results.

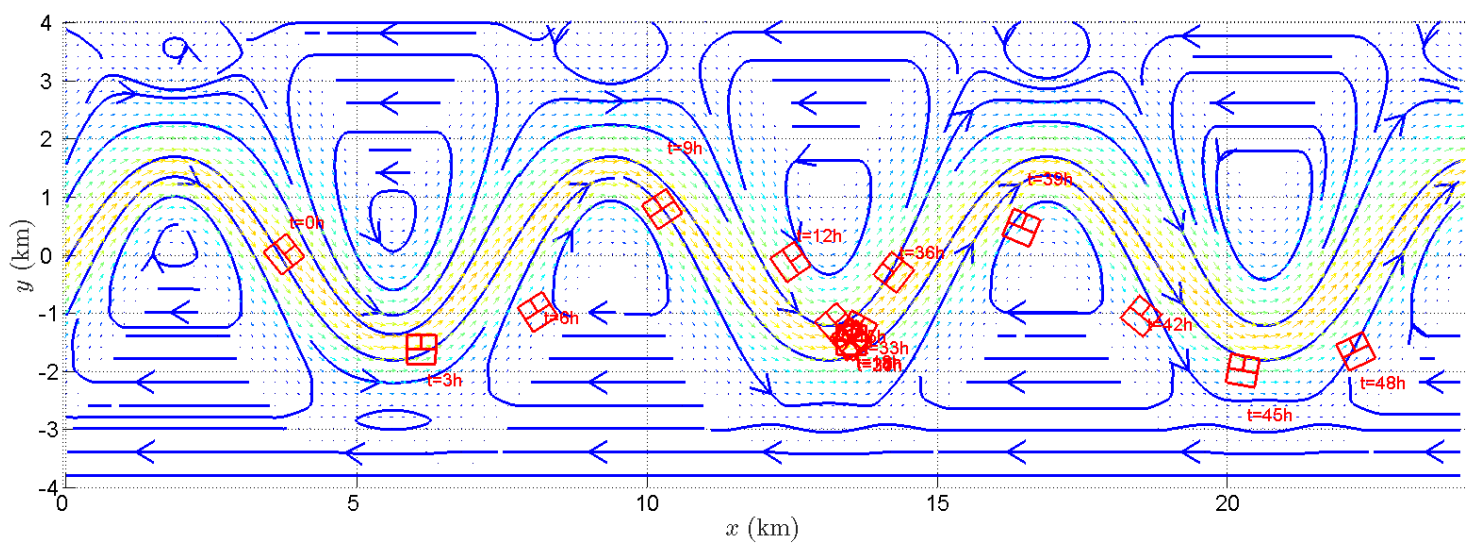

Figure 6.6: Drifting RoI tagging an initially jet-centred drifting feature at $t=[0,48]$ h. Shown alongside is the current velocity in the world frame, and comoving frame streamlines at $t=0 \mathrm{~h}$, $250 \mathrm{~m}$ depth.

\section{Coverage}

Figures 6.7-6.12 show the coverage proportion over time of the schemes. As expected, Scheme 1 is able to maintain the higher coverage proportion throughout the simulation. The scheme is even able to maintain a certain level of coverage by the end of the 
mission, while the coverage in those networks left purely to drift and DNR have fallen to zero. The coverage of the DNR scheme decreases rapidly due to the current velocity in the static frame of reference falling off away from the centre of the jet. This means that floats near the edges of the RoI move horizontally at a lower average speed per cycle, again considered in the static frame of reference, and are left behind by the RoI that is drifting at a faster speed in the current's core. Scheme 1 performs better because the trajectories it generates account for the current speeds at the float's horizontal location. The scheme finds the minimum displacement trajectory to a goal position inside the RoI. Because the water higher up is moving faster than the RoI and the water at greater depths is moving slower, this trajectory can at least zero the surge displacement so the node ends up at a surge ordinate within the RoI.

These trends are observed in the node positions for a representative run of each scheme at the start of each day, displayed in Figures 6.13-6.22. Scheme 1 is more effective at limiting the horizontal displacement of the nodes, keeping them clustered closer to the RoI. In the graphs, the RoI is the red cylinder.

A curious feature of Figures 6.7-6.12 is that the coverage declines haltingly. The coverage is steady at periods then drops rapidly, and these periods coincide with each other across the schemes. This occurs because of the particular current applied to the region. To explain what is transpiring, Figure B.39 was extended to show the position of the feature over 2 days. The resulting graph is displayed in Figure 6.6. Because of the fluid exchange caused by a time-varying meander amplitude, the tagged pollution alternates between being carried in the jet and escaping it (entering the vortex regime). When it leaves the jet, it enters a region where the current is virtually zero. It remains in this area until the propagating meandering jet passes it by, and entrains it once more in the jet. The first time the detrainment happens occurs around 15 hours and lasts until approximately $t=33$ hours. The entrainment time coincides with when the coverage rapidly declines. This is because as the current pattern passes by, not all the nodes are entrained at once. The nodes closest to the approaching meander are sucked up first, while the RoI, tagged at the centre, is still stationary. The former floats cannot prevent themselves moving forward, since the current is eastwards-only, move too far ahead of the RoI and become difficult to recover. The separation between these nodes and the centre of the RoI when it is picked up by the jet is too great, and the currents vary too much in between. Thus, the trajectories generated by Scheme 1 assuming that the current profile directly underneath each node applies everywhere do not produce the expected end positions. This is also why the coverage steadily decreases, as a portion of the network becomes 


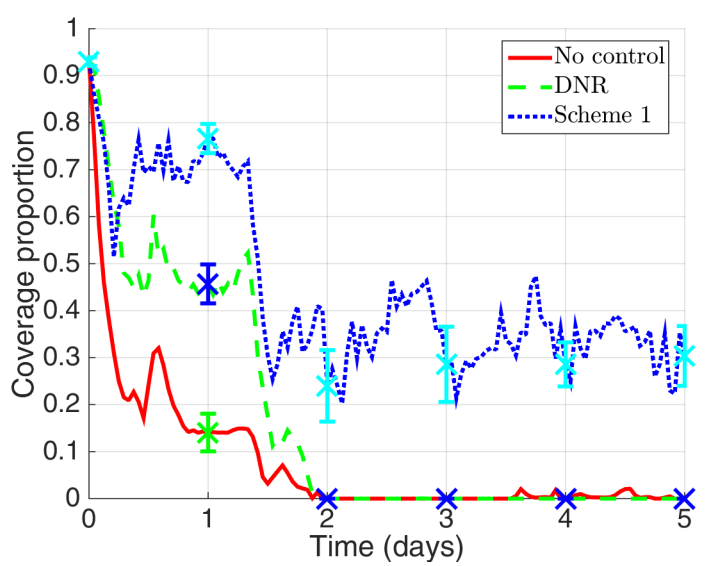

Figure 6.7: Coverage proportion over time. Network size: 40 floats.

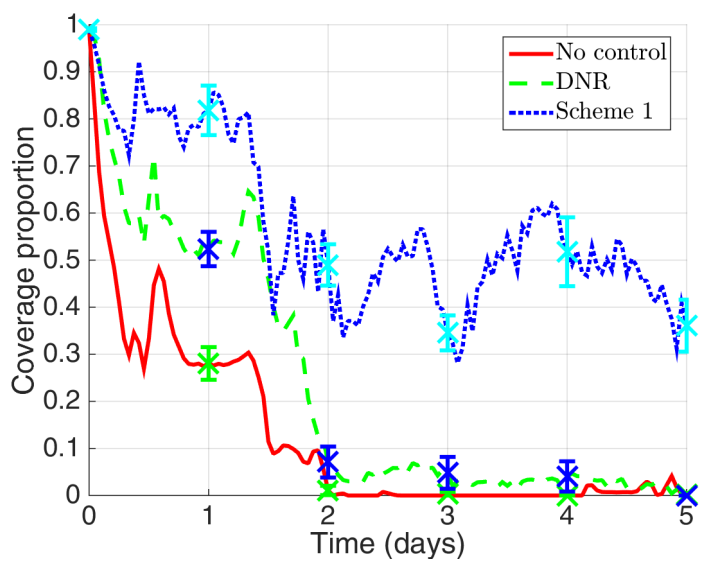

Figure 6.9: Coverage proportion over time. Network size: 80 floats.

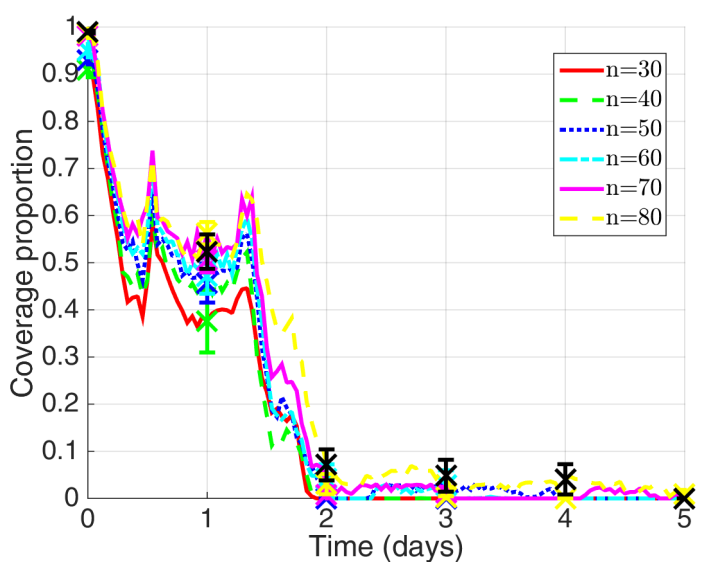

Figure 6.11: Dive and rise scheme: coverage proportion over time for various network sizes.

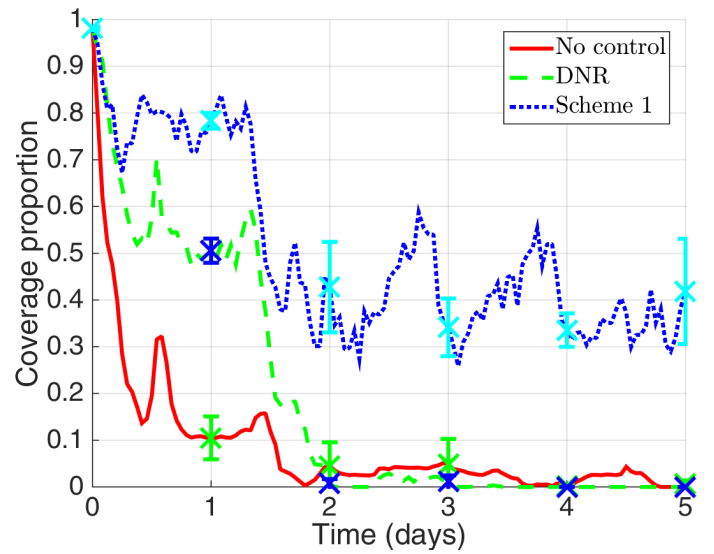

Figure 6.8: Coverage proportion over time. Network size: 60 floats.

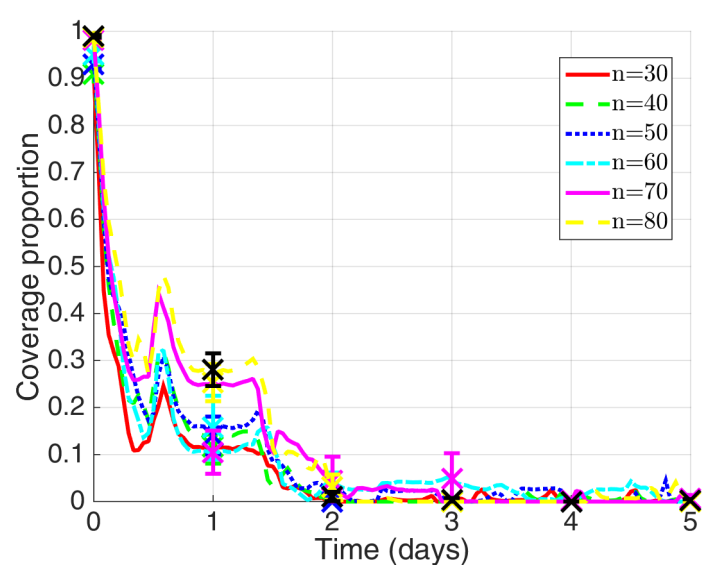

Figure 6.10: No control: coverage proportion over time for various network sizes.

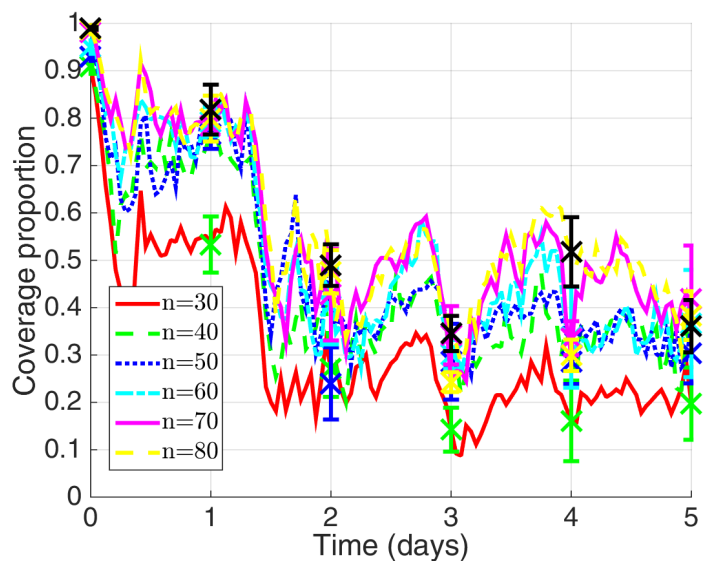

Figure 6.12: Scheme 1: coverage proportion over time for various network sizes. 
lost this way every entrainment.

Scheme 1 is not immune to this. The scheme only calculates a new trajectory on the node physically completing the last one, every $3-10$ hours. The trajectory is generated assuming that the current velocity below and above the float (measured by an ADCP) apply throughout the trajectory. When the node is outside the jet, these velocities are zero. However, when entrainment occurs the current is now the speed within the jet. The node is unaware of this. It continues on its preplanned velocity course, designed for a different current regime, and ends up in an unanticipated location, most likely some distance from the RoI.

As one way to improve the scheme and mitigate this effect, nodes should reevaluate the currents below and above them, and their trajectory to the goal on-the-fly rather than just upon surfacing. They can then react faster to changing current conditions. The convex optimization problem is trivial to solve, so this change would not incur much additional processing overhead. However, the energy cost of repeatedly emitting ADCP sound pulses may become significant.

The rapid decline at $t=0$ is the process of Scheme 1 stabilizing at an equilibrium solution, during which time the coverage is observed to undershoot. All nodes are initially located inside the deployment box and roughly uniformly distributed, giving high coverage. To maintain those positions at a later time, nodes must make a circuitous cycle across the height of the box. During this cycle they move out of the box. Only a percentage of the nodes is at the phase in their cycle where they return to their goal position and pass through the box. So only a proportion of the nodes, rather than all nodes, are within the RoI at any one time. This results in reduced coverage. 


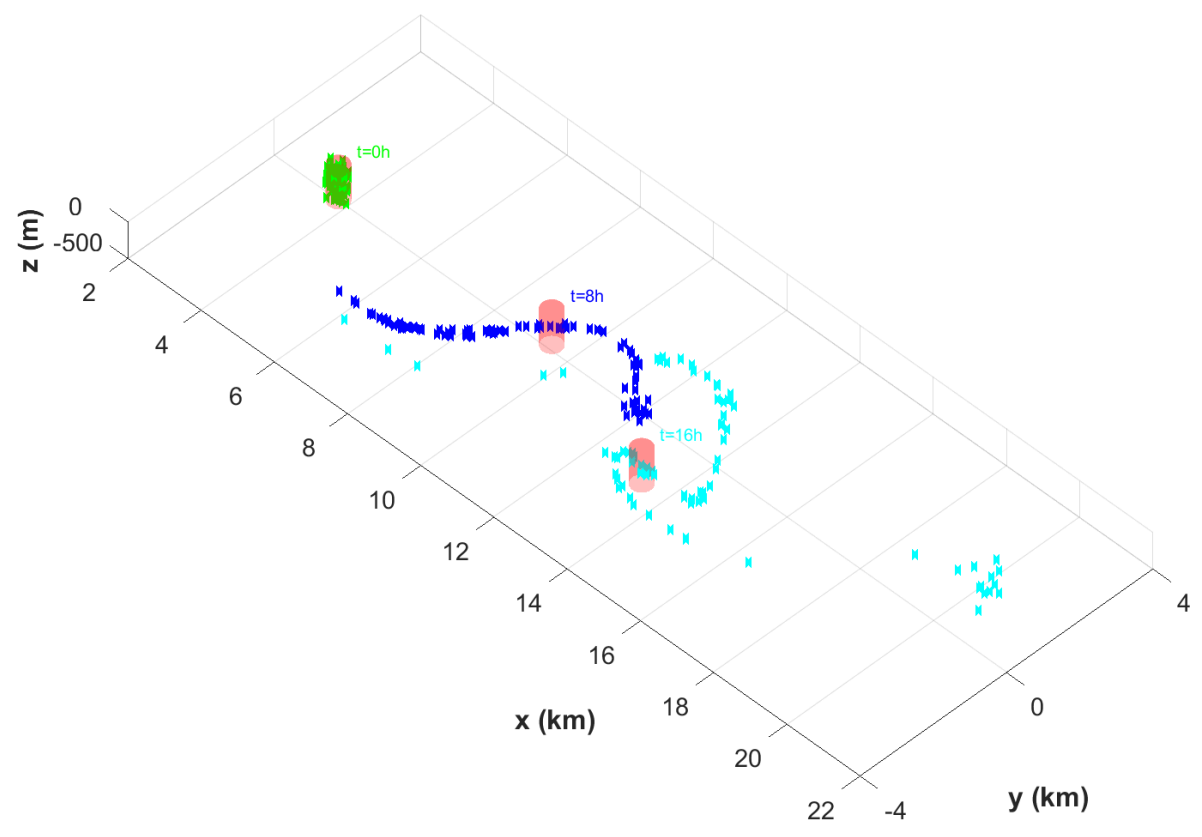

(a) No control

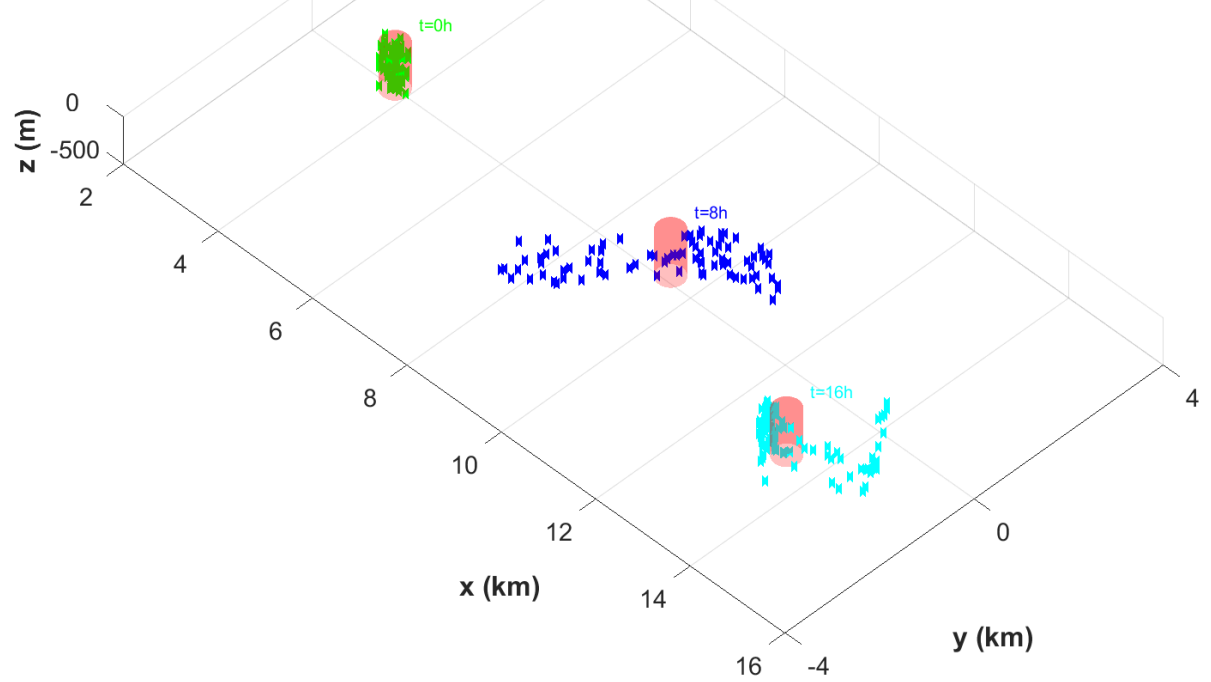

(b) DNR

Figure 6.13: Positions of nodes and RoI in the world frame at $t=0-1$ days for schemes: a) no control and b) dive and rise. 


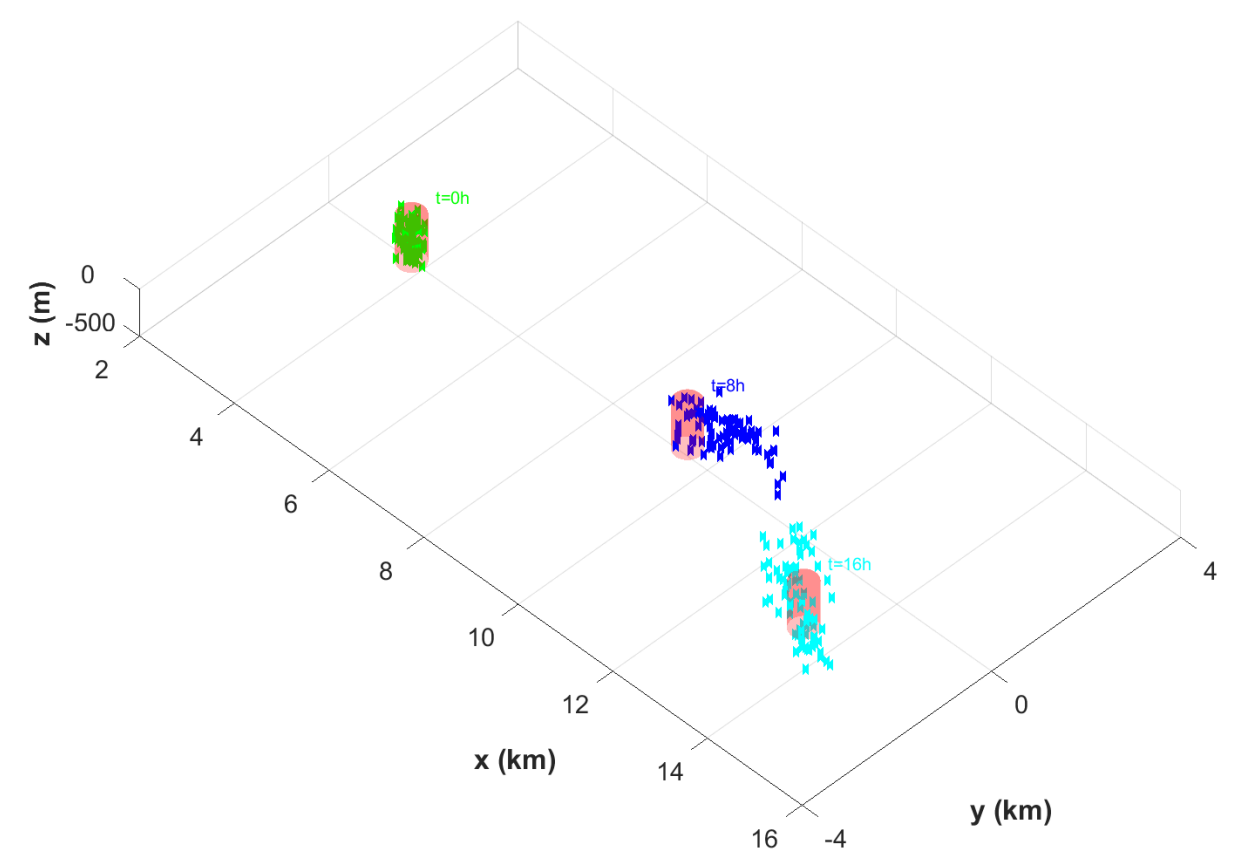

(a) Scheme 1

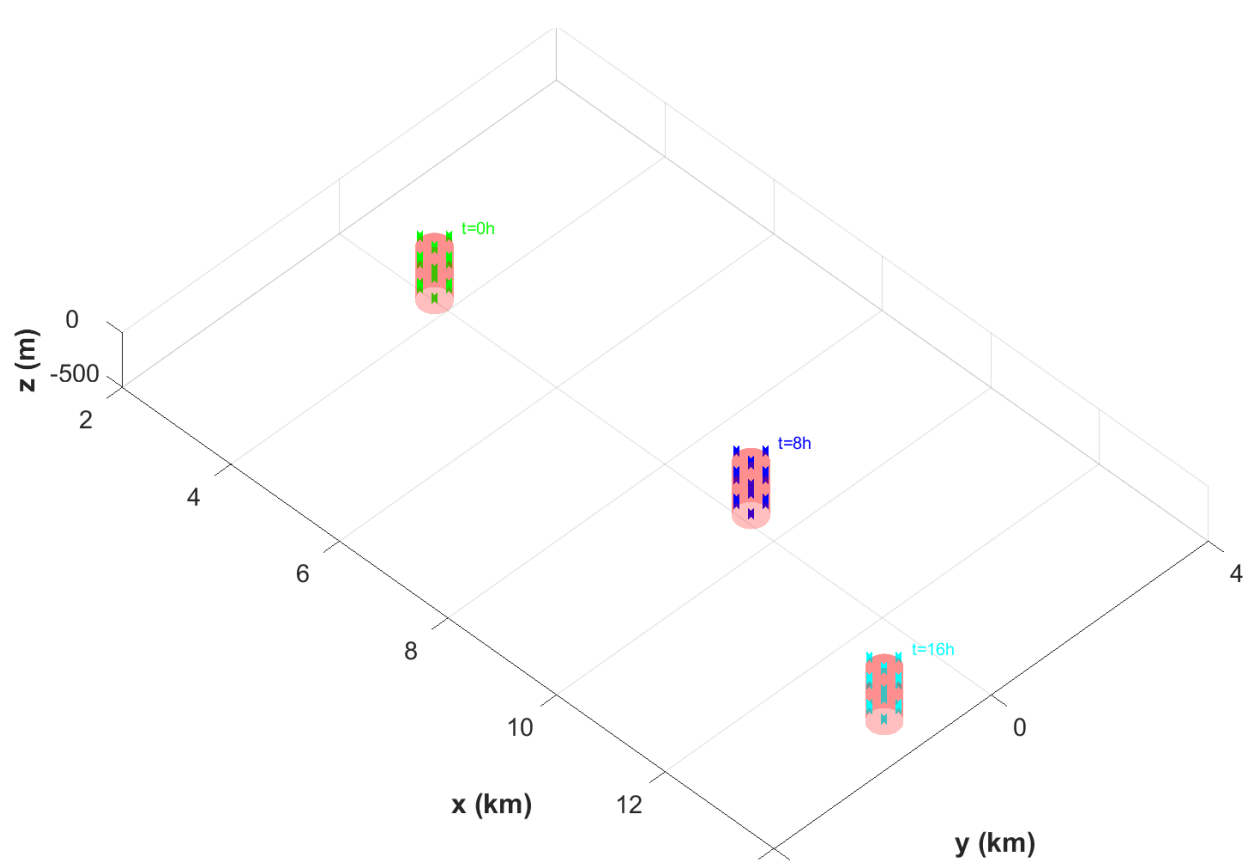

(b) AUV back-to-original scheme

Figure 6.14: Positions of nodes and RoI in the world frame at $t=0-1$ days for schemes: a) Scheme 1 and b) AUV back-to-original positioning scheme. 


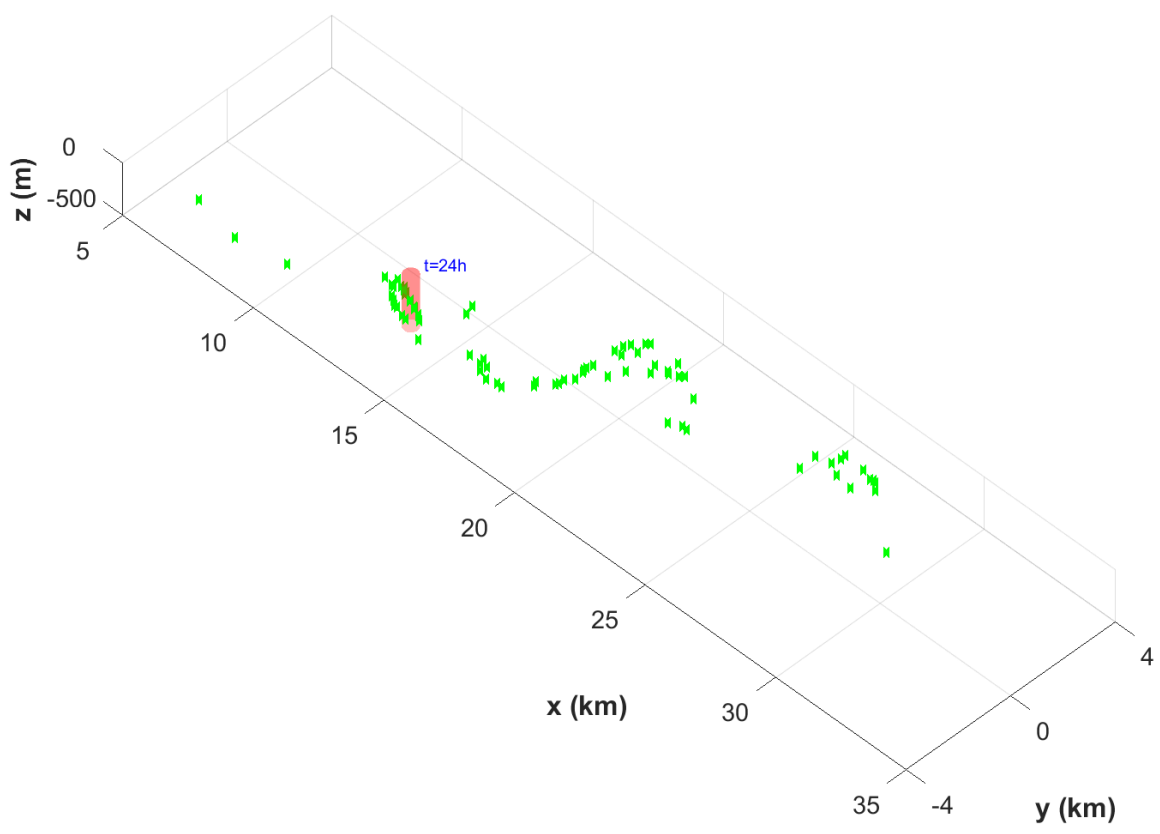

(a) No control

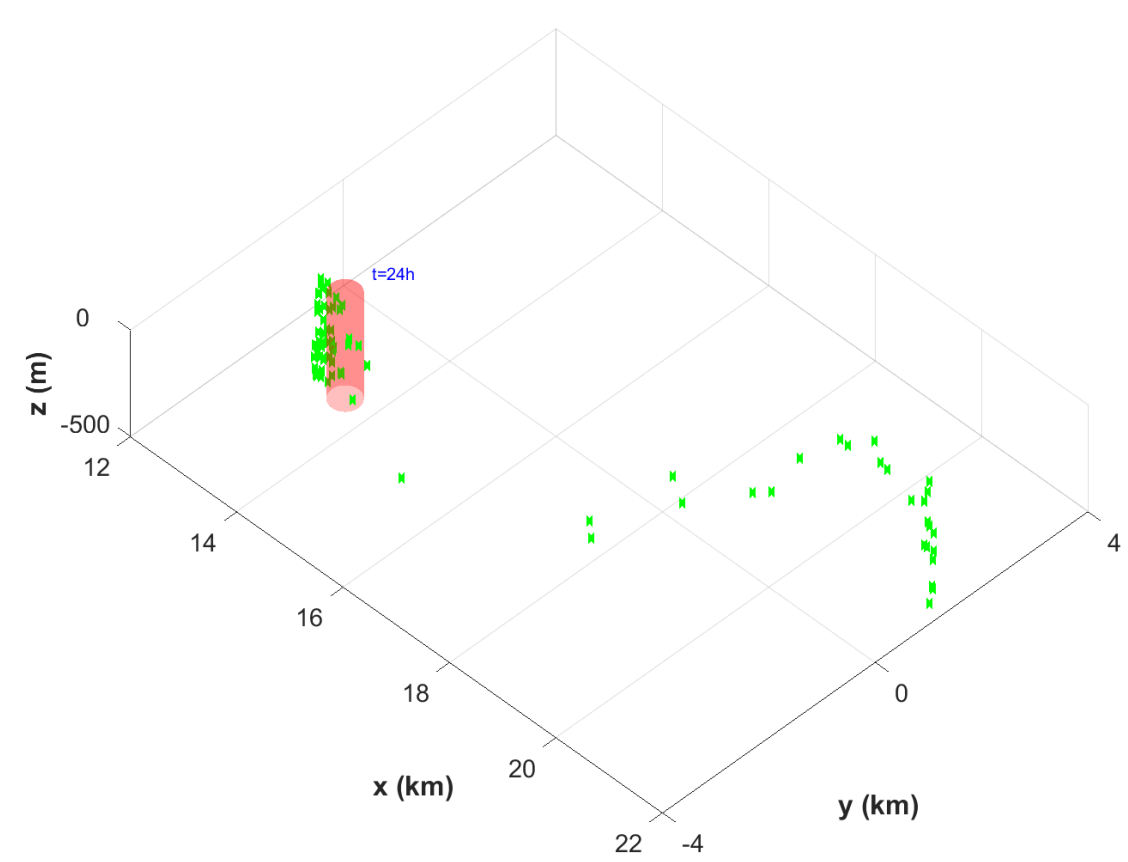

(b) DNR

Figure 6.15: Positions of nodes and RoI in the world frame at $t=1$ days for schemes: a) no control and $\mathrm{b}$ ) dive and rise. 


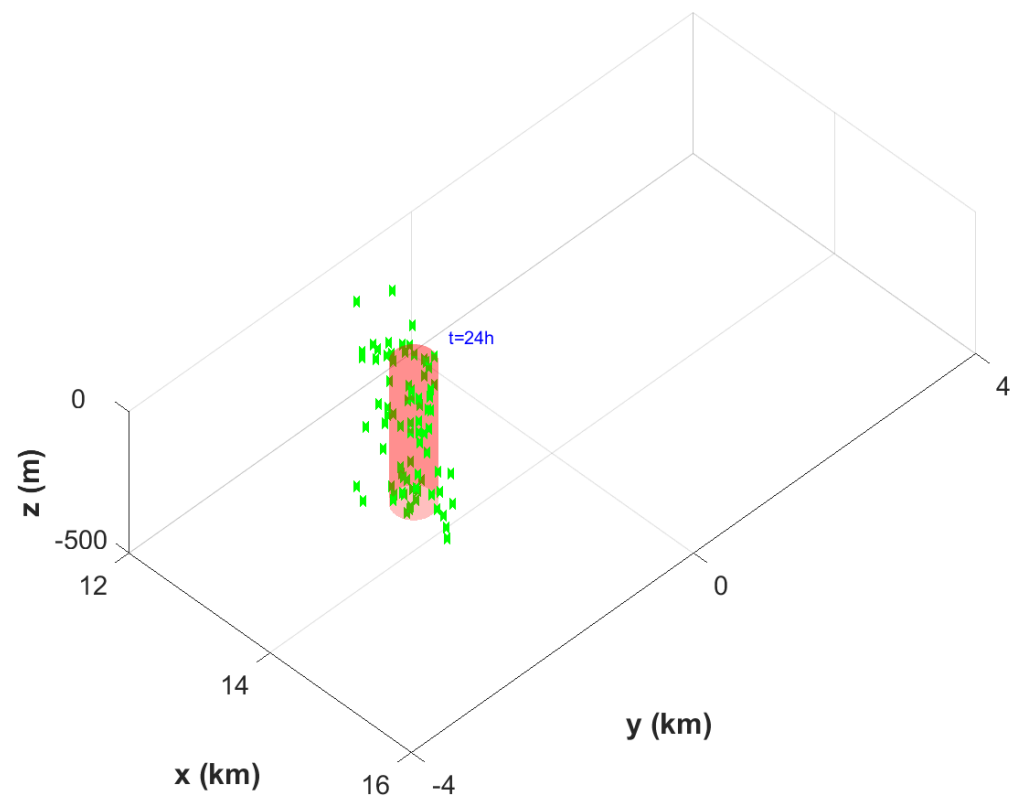

(a) Scheme 1

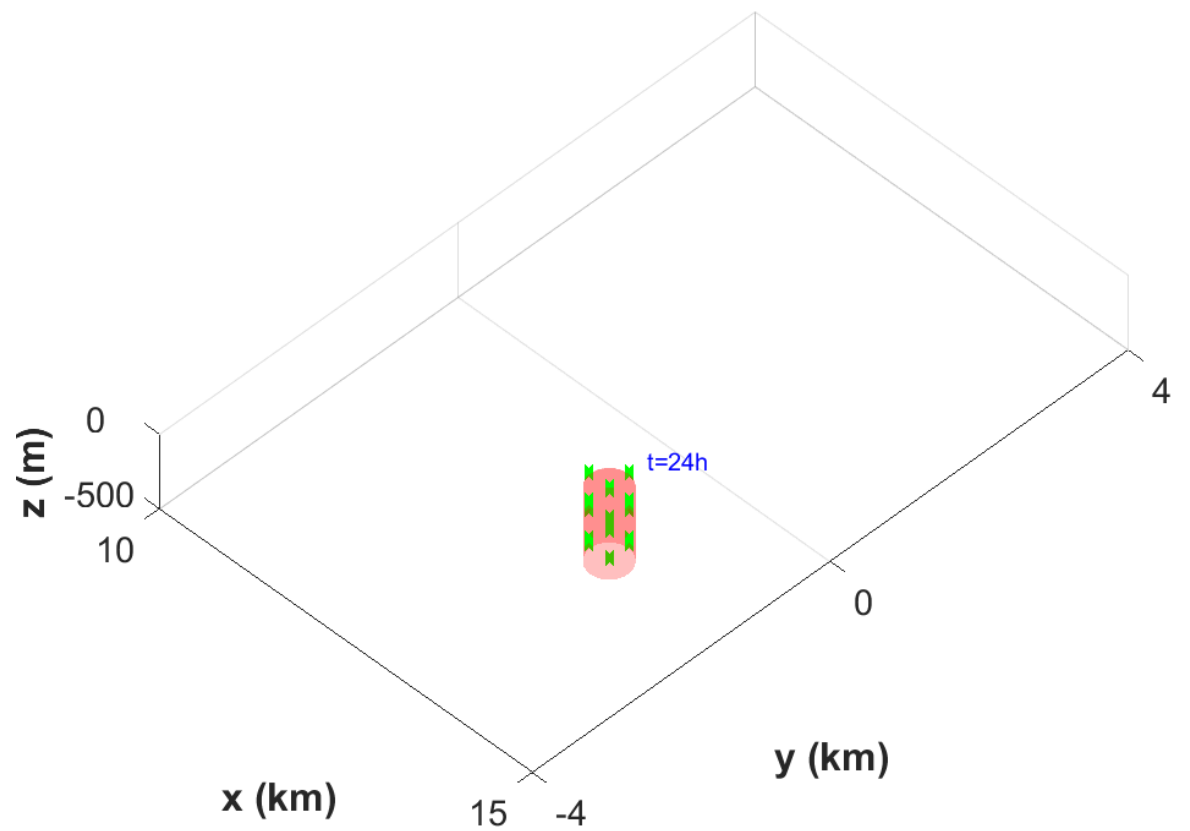

(b) AUV back-to-original scheme

Figure 6.16: Positions of nodes and RoI in the world frame at $t=1$ days for schemes: a) Scheme 1 and b) AUV back-to-original positioning scheme. 


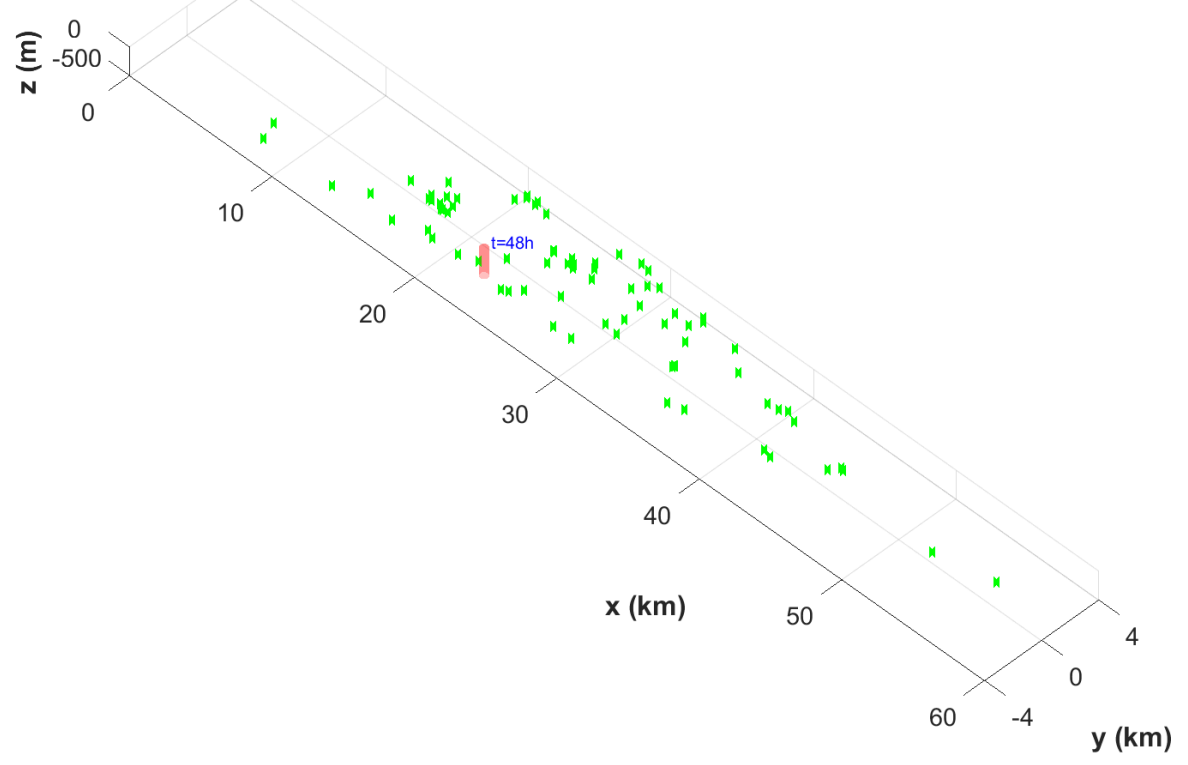

(a) No control

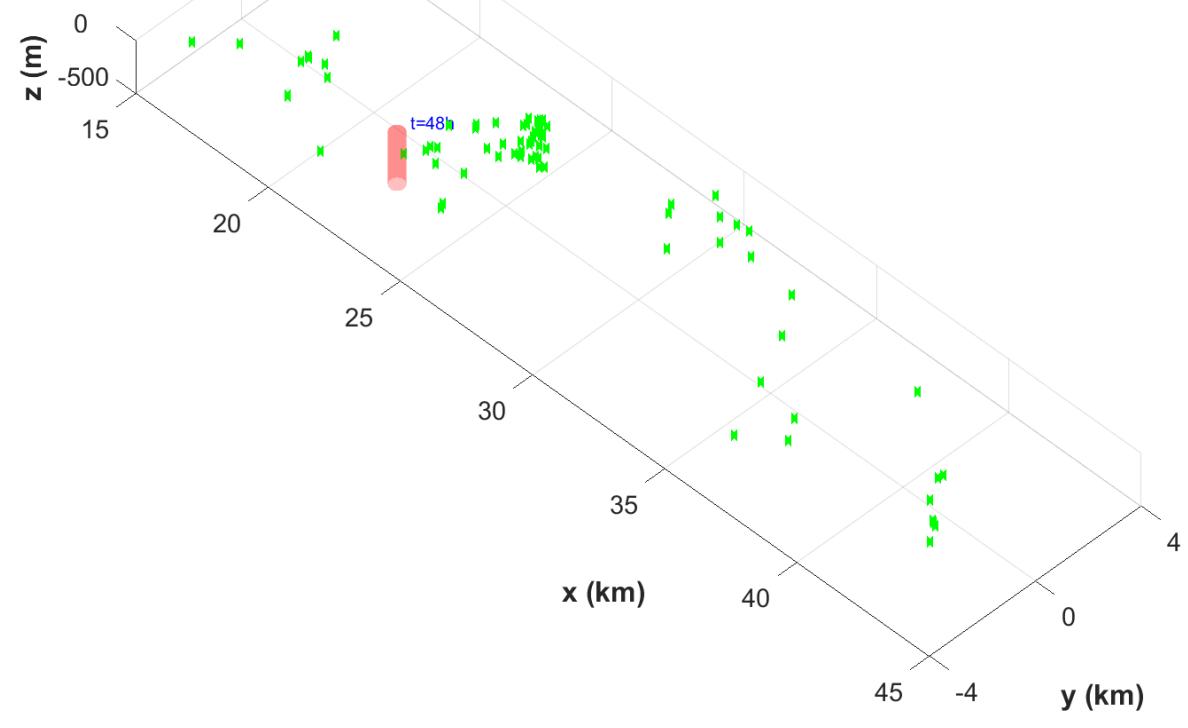

(b) DNR

Figure 6.17: Positions of nodes and RoI in the world frame at $t=2$ days for schemes: a) no control and $\mathrm{b}$ ) dive and rise. 


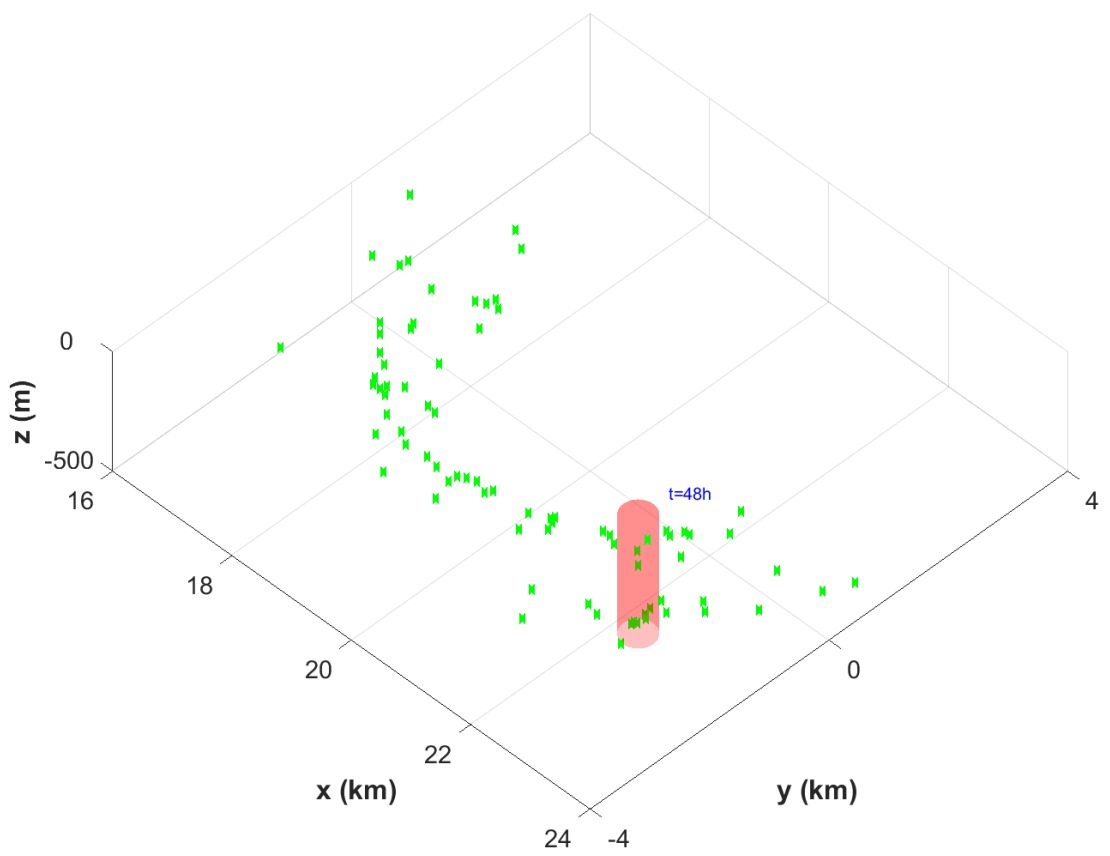

(a) Scheme 1

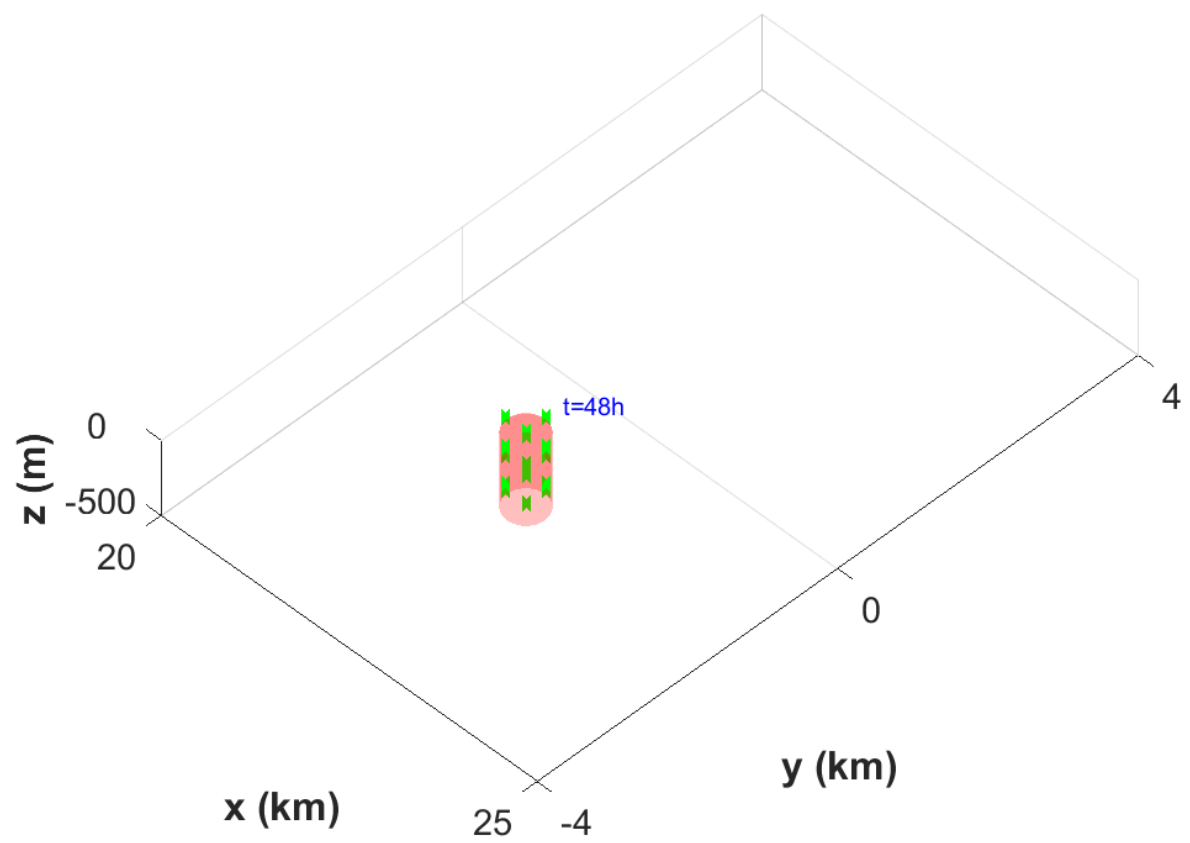

(b) AUV back-to-original scheme

Figure 6.18: Positions of nodes and RoI in the world frame at $t=2$ days for schemes: a) Scheme 1 and b) AUV back-to-original positioning scheme. 


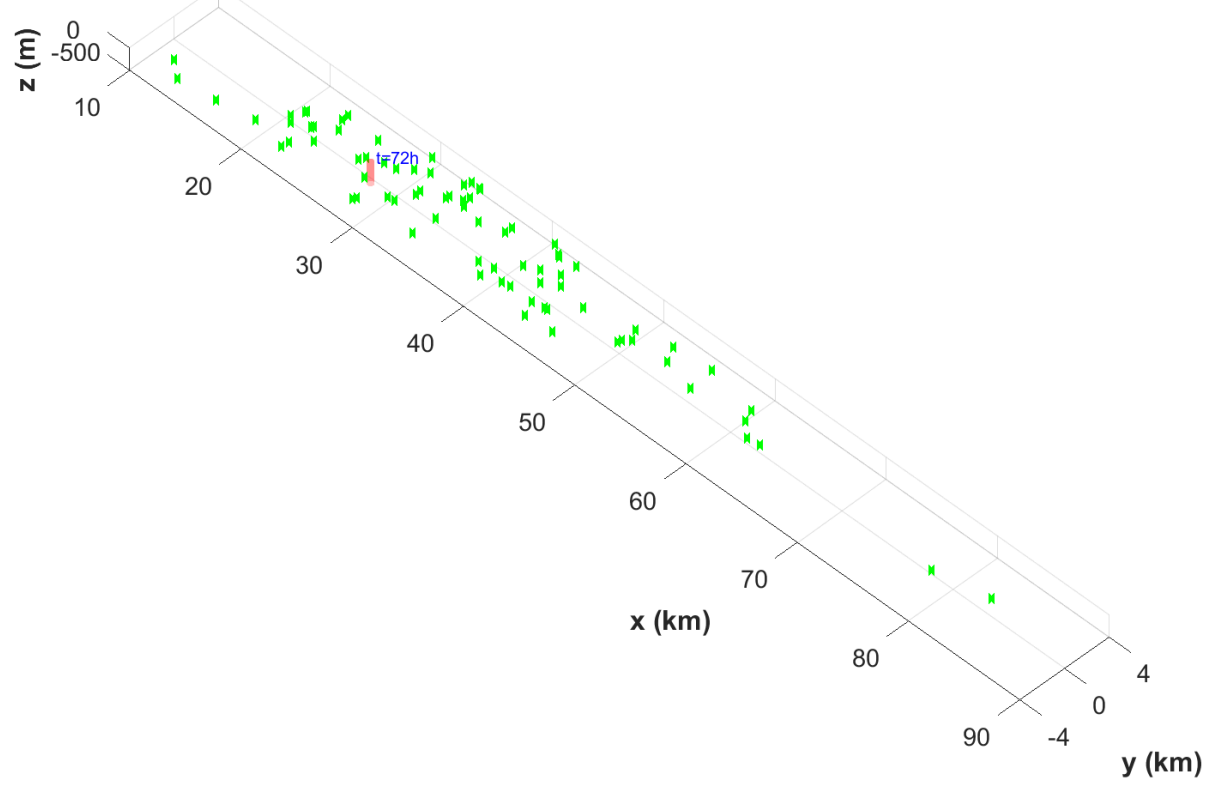

(a) No control

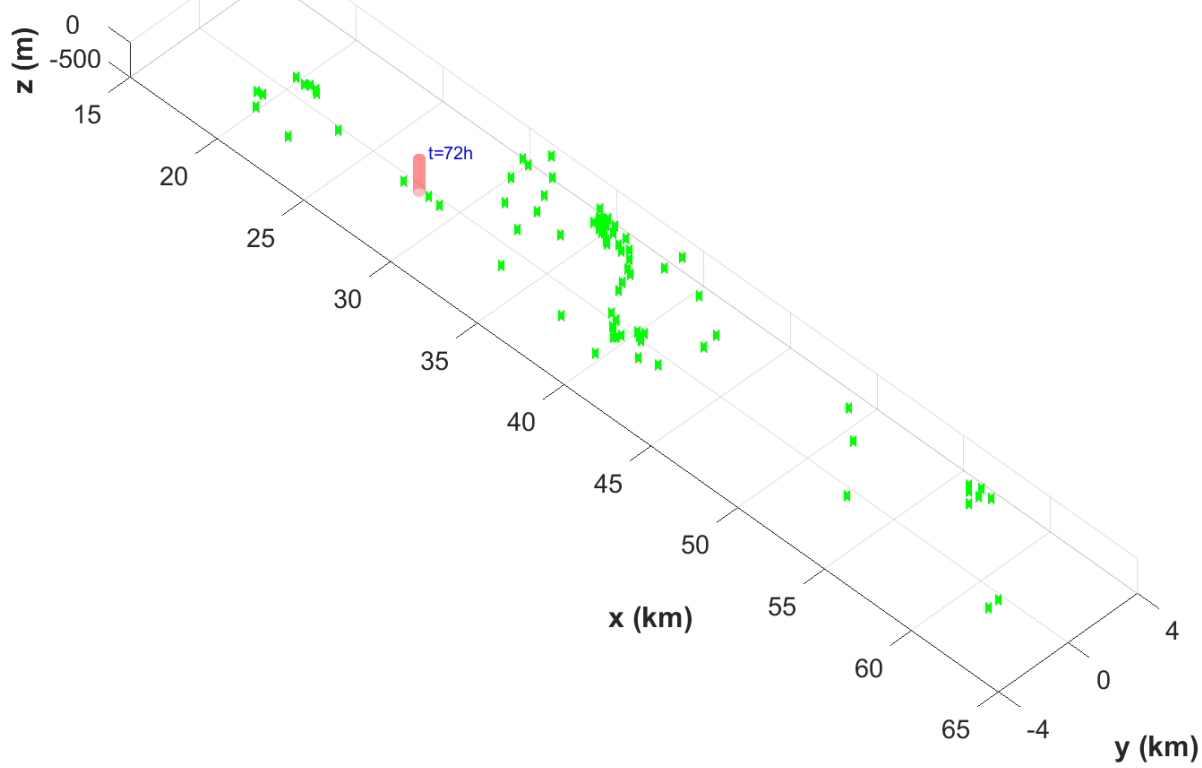

(b) DNR

Figure 6.19: Positions of nodes and RoI in the world frame at $t=3$ days for schemes: a) no control and $\mathrm{b}$ ) dive and rise. 


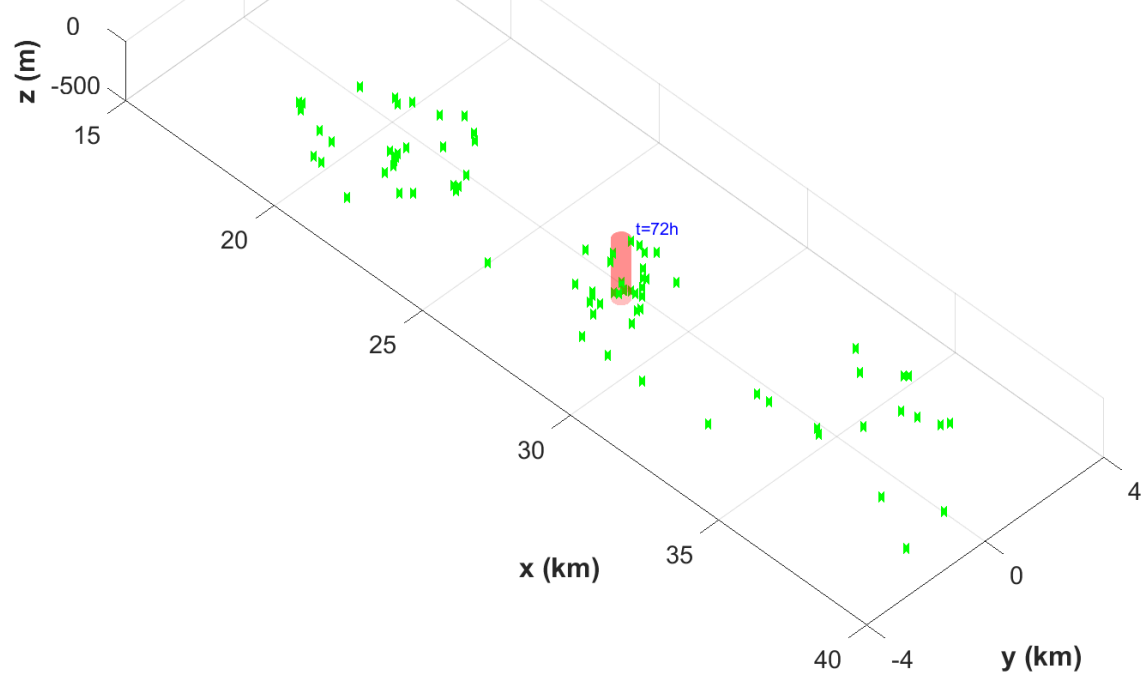

(a) Scheme 1

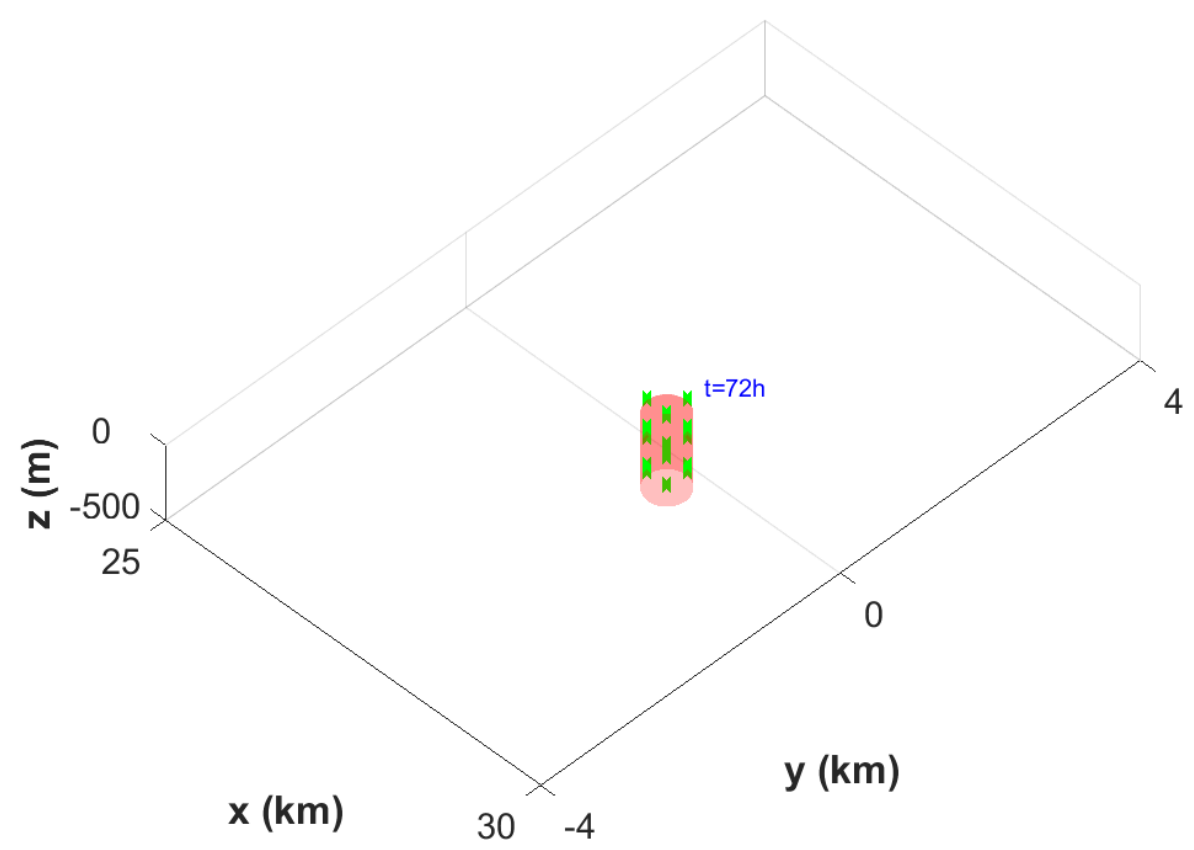

(b) AUV back-to-original scheme

Figure 6.20: Positions of nodes and RoI in the world frame at $t=3$ days for schemes: a) Scheme 1 and b) AUV back-to-original positioning scheme. 


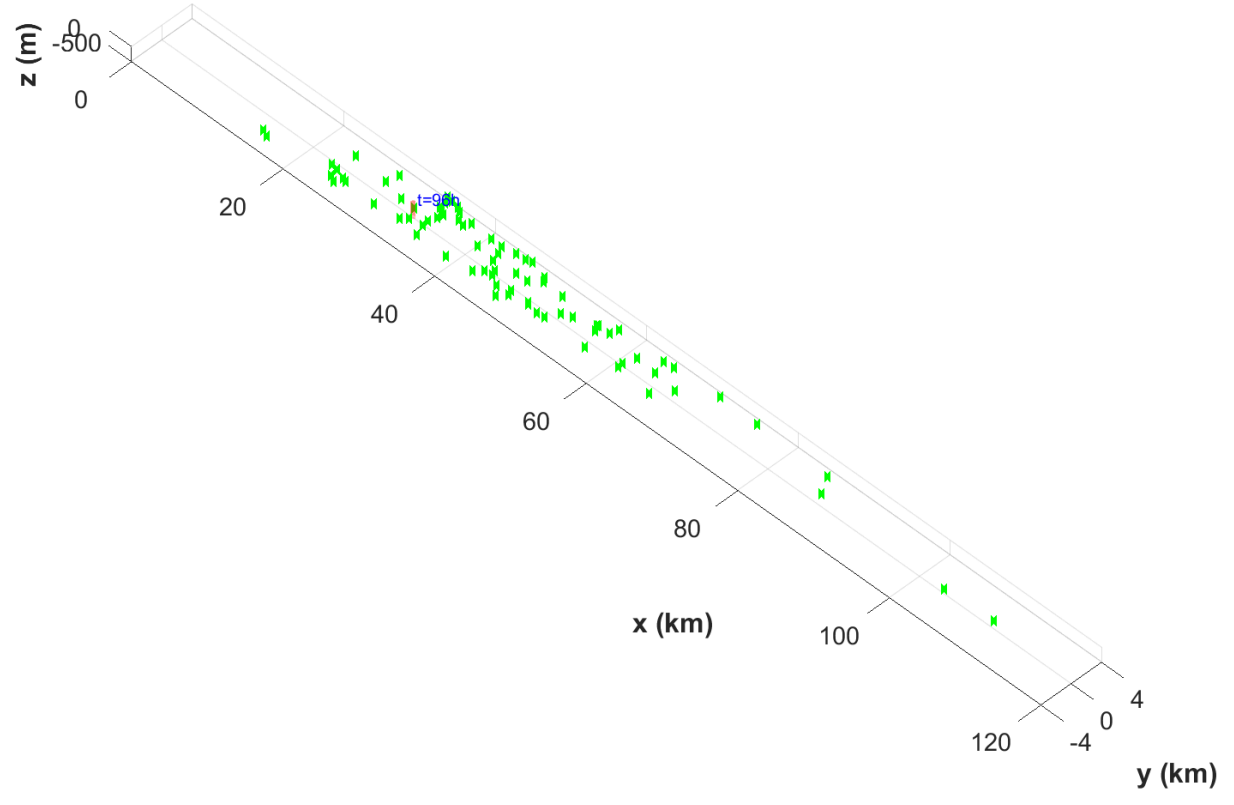

(a) No control

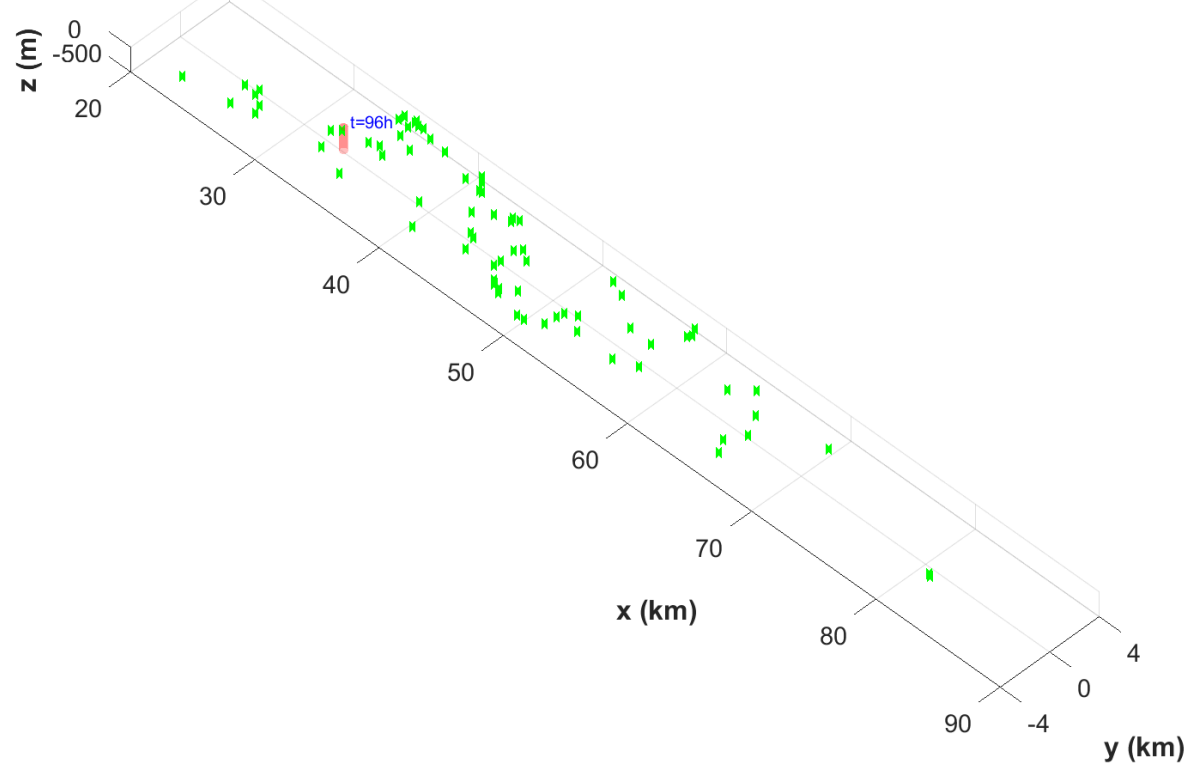

(b) DNR

Figure 6.21: Positions of nodes and RoI in the world frame at $t=4$ days for schemes: a) no control and $b$ ) dive and rise. 


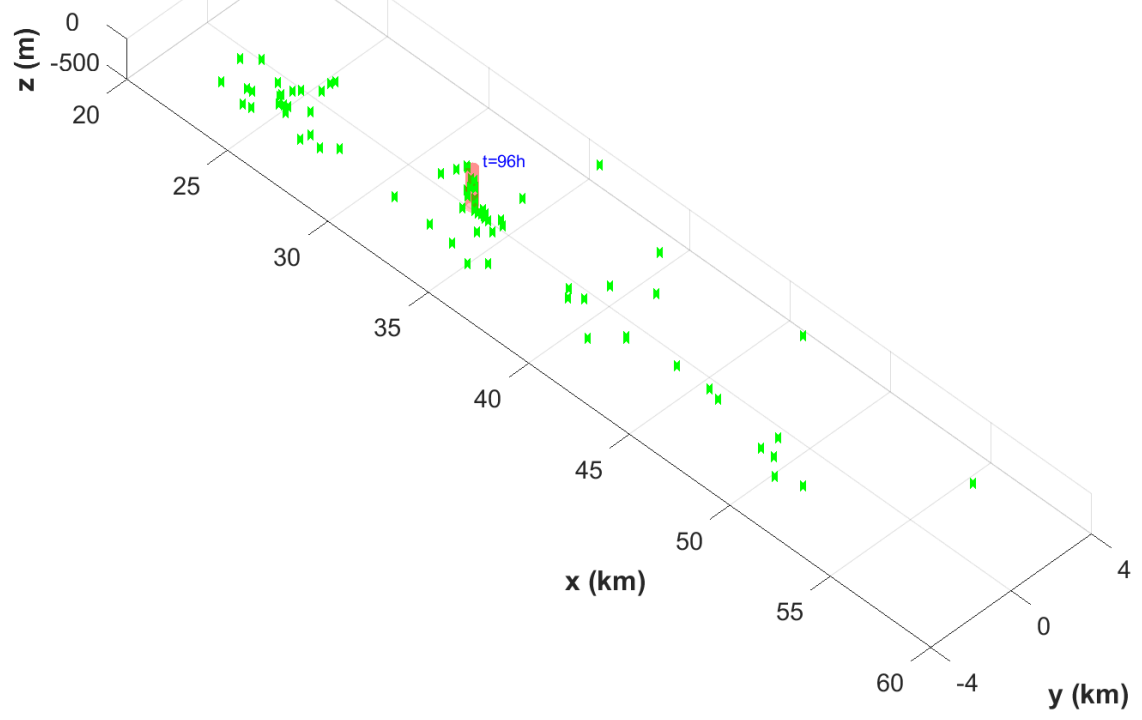

(a) Scheme 1

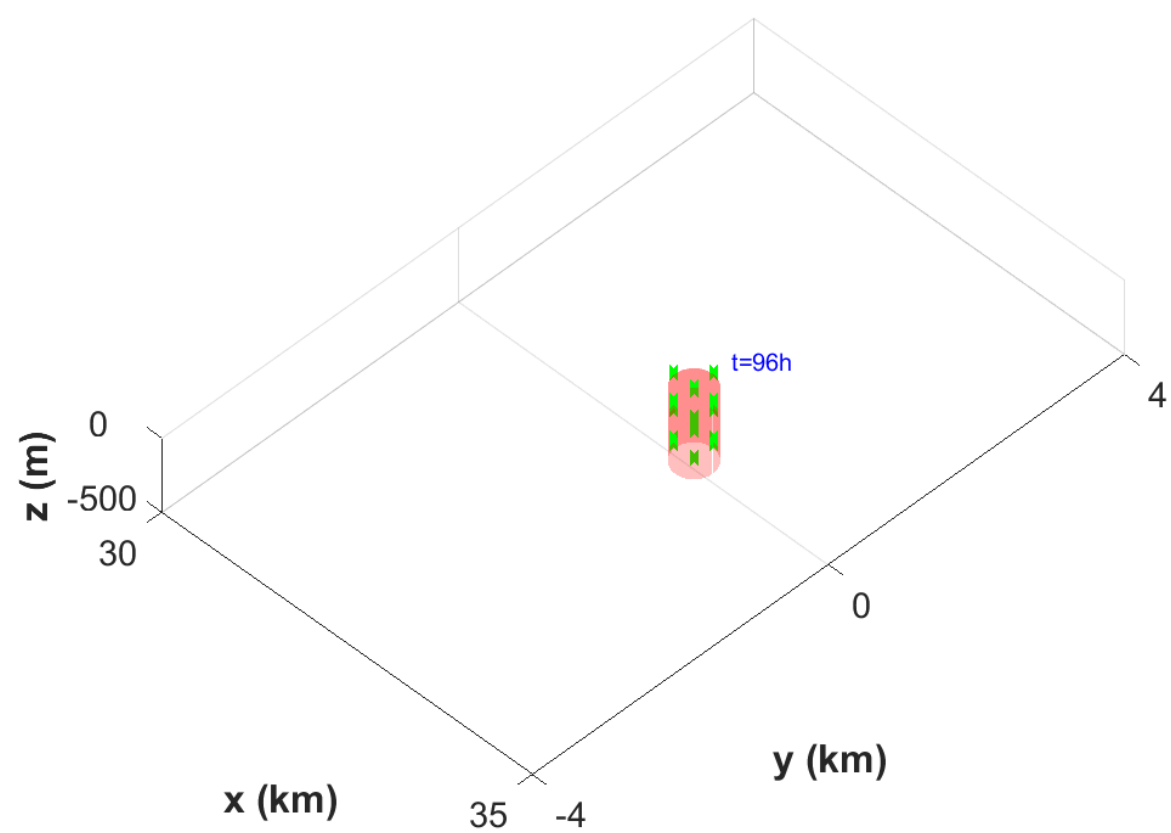

(b) AUV back-to-original scheme

Figure 6.22: Positions of nodes and RoI in the world frame at $t=4$ days for schemes: a) Scheme 1 and b) AUV back-to-original positioning scheme. 


\section{Electrical energy consumed in mobility}

The mean distances travelled by nodes under their own power are displayed in Figures 6.23-6.24 for a single representative run, and the mean energies consumed in mobility by nodes are plotted in Figures 6.25-6.26. The mean values are plotted along with histograms of distances travelled and energies expended by individual floats, to provide information on the energy balance among sensors [10]. As expected, the DNR scheme where all floats travel at maximum profiling speed covers the most distance and uses the most energy. Since the nodes are travelling at the same speed, the distance travelled is not distributed.

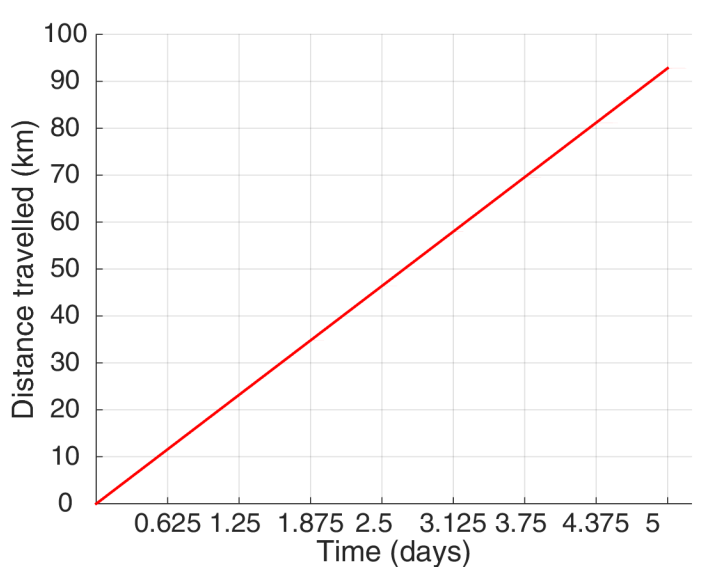

Figure 6.23: Dive and rise scheme: distance travelled by nodes under their own power against time in a run. Network size: 80 floats.

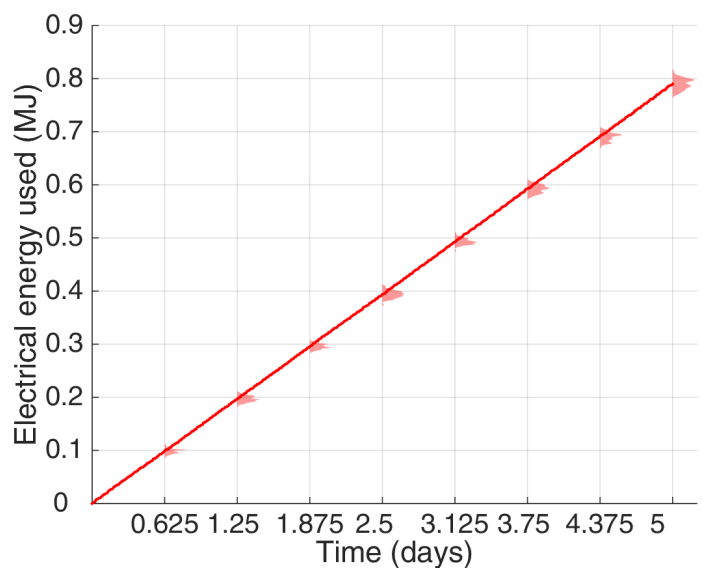

Figure 6.25: Dive and rise scheme: energy used in mobility by nodes against time in a run. Network size: 80 floats.

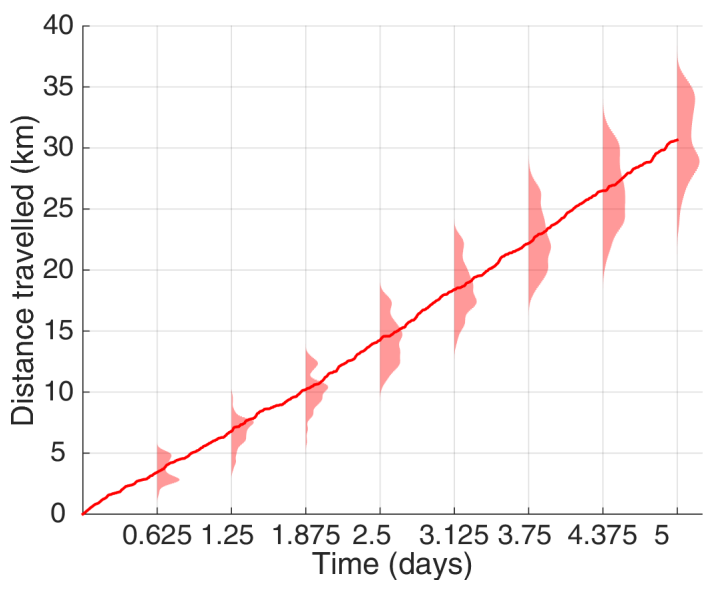

Figure 6.24: Scheme 1: distance travelled by nodes under their own power against time in a run. Network size: 80 floats.

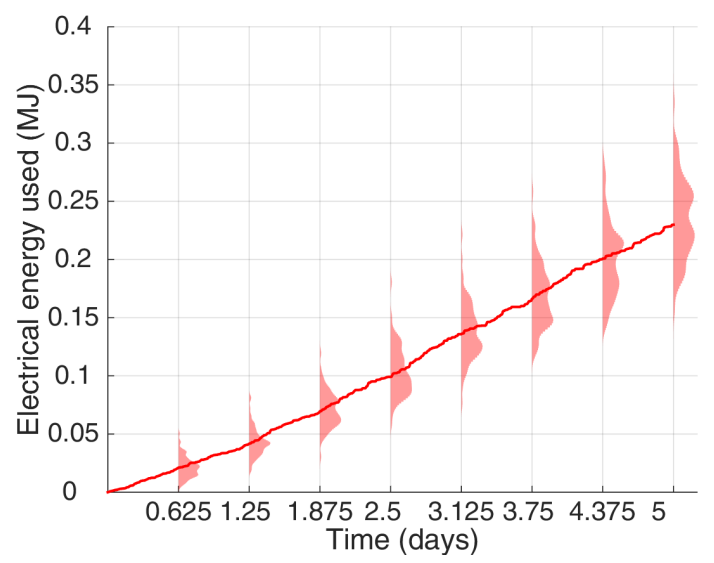

Figure 6.26: Scheme 1: energy used in mobility by nodes against time in a run. Network size: 80 floats. 
A concern is that at the end of the 5 day period, floats in Scheme 1 have expended a significant proportion (23\%) of their $1 \mathrm{MJ}$ power supply, the battery capacity of actual ALACE floats [47]. The devices running DNR have used up most of that supply, consuming $80 \%$ in the time period. So these schemes cannot be utilized for much longer duration missions. Note that these figures do not include the cost of communications and sensor hotel load, which also add an ongoing cost.

The energy usage is consistent with the data in [47], which gives the energy needed to surface from depths $100 \mathrm{~m}, 1000 \mathrm{~m}$ and $2000 \mathrm{~m}$ in under $1 \mathrm{~h}$. This is around the time needed to surface in DBR, $500 \mathrm{~m} / 0.2 \mathrm{~ms}^{-1}=0.7 \mathrm{~h}$. The energy for surfacing from $500 \mathrm{~m}$ lies somewhere between $5.9 \mathrm{~kJ}$ and $8.5 \mathrm{~kJ}$. Taking this to be the midpoint, $7.2 \mathrm{~kJ}$, over 5 days in the DBR scheme the float cycles 86 times. So utilizing the designer's figures as well in a back-of-the-envelope calculation, the float will have consumed 0.62 MJ energy at the end of 5 days and almost exhausted their $1 \mathrm{MJ}$ battery capacity as well. The close correspondence of this value with the $0.8 \mathrm{MJ}$ value our simulator calculated provides support for the correctness of our profiling float energy model.

\section{Hybrid AUV-float networks}

The performance of the hybrid AUV-float networks is now presented. Figures 6.276.29 portray the coverage of the network using Scheme 1 for different float network sizes and with 1, 3 and 5 AUVs in support. Figure 6.30 displays the mean number of additional AUVs required to obtain $75 \%$ coverage up until certain times, computed from each run as $\sum_{i=1}^{k} m\left(t_{i}\right) / k$, where $k$ is the index of the last sample prior to the simulation time limit and $m_{i}$ is the number of AUVs needed to obtain $75 \%$ at time $t_{i}$.

These figures demonstrate that with a few AUVs only in support, as a proportion of the float population size, a relatively high though not full level of coverage can be maintained throughout the mission duration. The floats do the best they can to cover the RoI, even though the positions they can reach are limited by what currents are present. The AUVs move to coverage holes that develop when the flows there make them impossible to access, for example because the currents diverge from there. The number of AUVs needed to obtain 0.8 coverage over the entire mission duration ranges from 6-8 for networks of 30-80 floats, derived from Figure 6.30. In typical cases, as shown in Figures 6.27-6.29 for a reasonable standard of coverage, 0.6 or more, 3 AUVs are needed. The coverage provided by floats accounts for around half the value in this case, which is provided at much reduced component cost. 


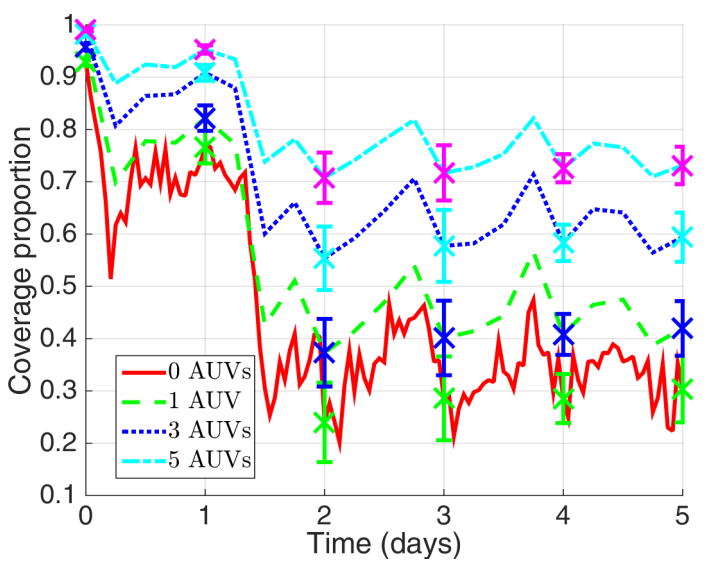

Figure 6.27: Coverage proportion over time for Scheme 1 with 1,3 and 5 AUVs present. Network size is 40 floats.

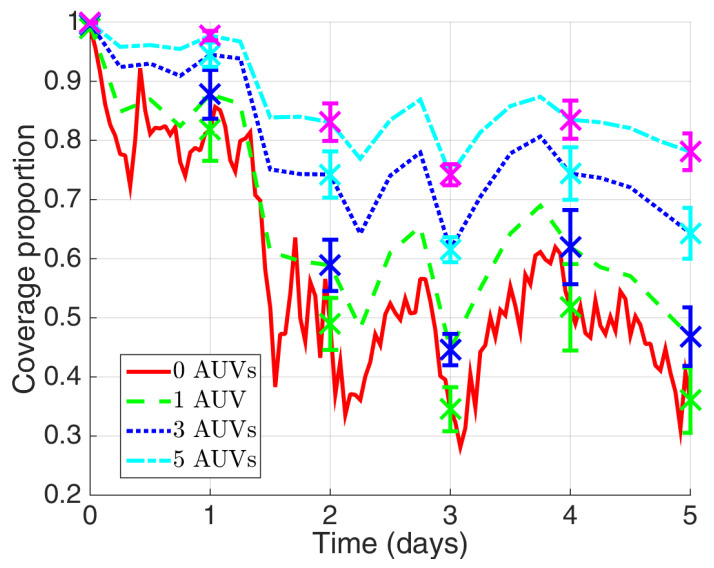

Figure 6.29: Coverage proportion over time for Scheme 1 with 1,3 and 5 AUVs present. Network size is 80 floats.

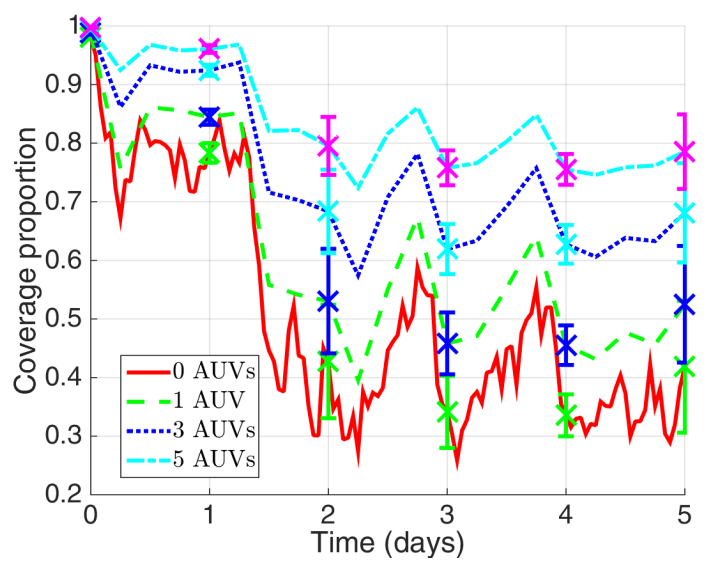

Figure 6.28: Coverage proportion over time for Scheme 1 with 1, 3 and 5 AUVs present. Network size is 60 floats.

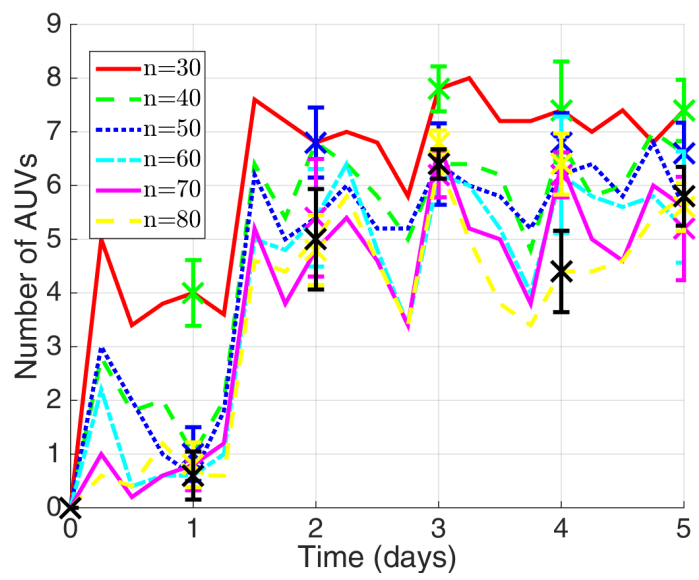

Figure 6.30: Mean number of AUVs that need to be added to obtain $80 \%$ coverage up until various times, against the float network size.

Normalized cumulative coverage against deployment cost tradeoff graphs of the various schemes are produced in Figure 6.31-6.33. The coverage of the AUV-only scheme is the result of greedily adding nodes to positions in the minimal truncated octahedron lattice required to fully cover the RoI. The lattice is initially empty, and a place in the formation is occupied if of all the lattice positions it increases the coverage the most. The normalized cumulative coverage for this scheme is the same as the instantaneous coverage at all times. The networks that adopt Scheme 1 that we have proposed, either with or without AUV support, consistently appear to the left of the frontier set up by the AUV-only graph. This means that for the same level of coverage, our scheme can provide it with reduced deployment cost compared to 


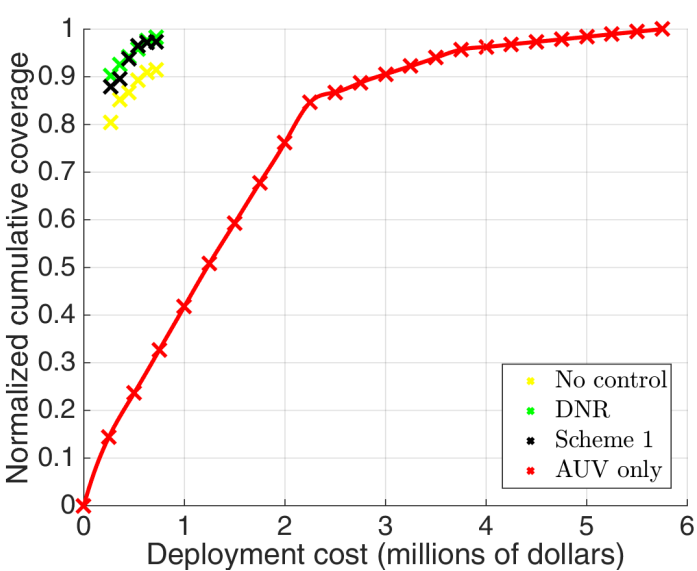

Figure 6.31: Tradeoff graph of the normalized cumulative coverage performance of schemes up until $t=1 \mathrm{~h}$ against deployment cost.

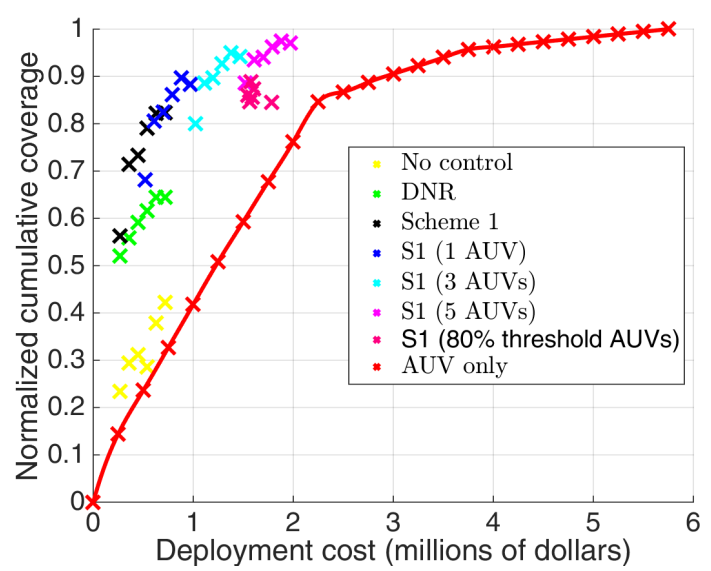

Figure 6.32: Tradeoff graph of the normalized cumulative coverage performance of schemes up until $t=1$ days against deployment cost.

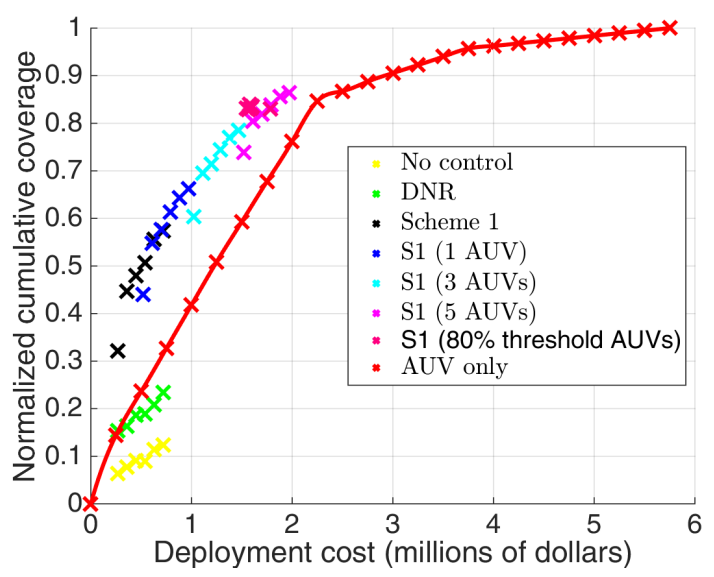

Figure 6.33: Tradeoff graph of the normalized cumulative coverage performance of schemes up until $t=5$ days against deployment cost.

these use of AUVs only. This advantage degrades with the length of the simulation as more floats escape from the system and cannot find a set of currents that allow them to return to the RoI. With no control and DNR, after 5 days the AUV solution becomes preferable in both coverage and cost.

\subsection{Summary}

In this chapter, we evaluated the performance of our float movement scheme for extending coverage in a simulated Lagrangian tracking pollution monitoring scenario. Current 2, our physically-inspired 3D coastal current mobility model, was employed 
as the flow in the scenario, and the parameters of the floats were based after the widely-used ALACE floats in order to make our simulations as realistic as possible. The Lagrangian tracking scenario involved monitoring a drifting fixed-size patch of pollution, modelled after an underwater oil plume. The performance of our Scheme 1 was measured by the cumulative coverage of the RoI over the simulation duration, the coverage achieved against the node deployment cost, and the electrical energy consumed, and compared with a simple dive-and-rise float-based node movement scheme, an AUV-based node movement scheme and employing no mobility. After 5 days, the average ALACE float implementing Scheme 1 has used up 23\% of its total energy supply in mobility, and our scheme is found to use $350 \%$ less energy than the dive-and-rise scheme. The results demonstrate that our scheme provides coverage more cost-effectively than utilizing AUVs only to track the RoI. Unlike the dive and rise scheme or having the floats perform no profiling, which result in zero area coverage after two days, our scheme is able to provide an ongoing level of coverage of the region to be monitored, of around $0.3-0.5$ as a proportion of the RoI depending on the network size. 


\section{Chapter 7}

\section{Conclusion and future research}

In this research we posed the problem "how well do we know the underwater currents for mobile UWSN applications?", in order to answer the essential question "how viable is it to employ drifting UWSNs, consisting of untethered nodes that are advected by the currents but otherwise do not propel themselves through the water, for monitoring?" The potential of such networks was raised in [41], leading to the development of routing [112,113,134,205] and localization protocols [58] for them. Using inexpensive, recoverable drifting nodes, large numbers can be rapidly deployed (air-dropped [112]) to a time-critical phenomenon, such as the immediate aftermath of an oil spill or a transient HAB, and follow the feature as both drift with the currents.

As surveyed in Chapter 1, it turns out we know very little about how these networks drift. Existing 3D models for the underwater current are unrealistic or deficient. These have been either stochastic models adapted directly from those employed in simulations of land-based networks, such as random waypoint and random walk [30,119], or are derived from numerical ocean model current forecasts [42,130]. In the latter case, the forecast velocities are too coarsely resolved to adequately reflect the neighbourhood interactions between nodes, as well as being inaccurate, which was described in Section 5.1.5. Recently, a physically-inspired current mobility model known as MCM has been proposed. This is only defined along the water's surface, in 2D, however, and 3D extensions of this model have been simplistic $[57,181]$, arbitrary [14] and likely flawed.

UWSN protocol schemes have been evaluated exclusively employing these models. This raised the question, stated at the top. How can the performance, and hence viability, of 3D drifting UWSNs, have been accurately represented when they have not been validated in realistic 3D underwater mobility? The currents especially 
affect the long-term performance of UWSNs. UWSNs require nodes to be present at all horizontal layers beneath the ocean, in order for data at deeper levels to be relayed upwards to the surface. Currents have been generally found to decrease in strength with depth $[48,185]$, however, so over time nodes at greater depths will tend to be left behind and disconnected from the faster moving nodes on the surface that have progressed further along horizontally. This is not represented in existing 3D models $[113,119,204,205]$, where current speed is independent with depth.

In Chapter 3, we formulated a realistic 3D Gulf Stream mobility model based on MCM. The velocity structure of our model, which agrees with measured profiles of the Gulf Stream, is a more accurate representation of the flow than existing MCM-based models. We also proposed a mobility model for coastal currents by scaling our Gulf Stream model, although its accuracy is likely to be limited due to coastal currents arising from different oceanic process than the Gulf Stream. To the best of our knowledge, these are the first physically-inspired 3D current models developed for UWSN research. Using our coastal current model (Current 2), the connectivity of a 3D drifting UWSN and performance of two pressure routing protocols, DBR and HydroCast, were studied in simulation in an oil plume monitoring scenario in Chapter 4. Pressure routing is considered a promising approach to routing, which takes advantage of the vertical structure of UWSNs to avoid expensive flooding and localization needed by other schemes. Previously, the averaged performance over the entire simulation running time had been given for these schemes and they had been simulated in unrealistic land-based and depth-invariant current mobility models. Their performances over 3 hours and at various periods within this interval were evaluated. In our study, the results showed that connectivity and the ability of routing protocols to successfully deliver packets degraded over time. The lower speed of deeper layer waters causes the network to shear apart. The nodes at depth lag behind upper layer nodes, and move out of communications range of the relays they need to deliver data to surface sinks. In one case, beginning from an almost fully connected deployment, no nodes were connected after 3 hours of drift.

The implication of these findings is that some means of keeping the network connected and within the RoI, to cover it, is needed. This can be either by redeploying nodes, for instance by using AUVs to pick up and relocate nodes [119], deploying additional sensors into the RoI over time [32] or equipping each node with its own mobility. This resulted in a new research question being formulated "how can the lifetime of untethered 3D UWSNs be extended using node mobility?", and an initial scheme for extending the operational lifetime of a UWSN by controlling the self- 
propelled motion of nodes so that they would return to cover the same part of the RoI is proposed in this thesis.

It is expensive to provide omnidirectional mobility via nodes that are AUVs however, so a network of profiling floats is considered. Maintaining full coverage with AUVs is also trivial, in the sense that these vehicles can at each time simply move counter to the currents to remain at a fixed position covering the RoI. Improved schemes have also been proposed for AUVs, where holding positions are exchanged between vehicles so that energy usage is balanced [120]. Coverage maintenance with floats is a more interesting problem, control-wise since these devices can only profile up and down but by doing so can move horizontally via exploiting different currents at different depths. Float-based UWSNs could potentially prove a superior solution to AUV UWSNs, given an appropriate movement scheme and current conditions, by also being able to move in $3 \mathrm{D}$ to cover the same positions as AUVs, while doing so with lower deployment costs.

This is the first time that float motion planning has been applied to the problem of 3D UWSN RoI coverage. Previous works had examined 2D path planning for single floats $[93,181,182]$ and 2D formation control of float arrays [42, 130], The devices were assumed to profile with infinite vertical velocity, and the energy used in mobility was not considered. In our 3D coverage problem, floats need to be positioned accurately on to coverage holes less than the node's sensing range $\approx 100 \mathrm{~m}$ wide. This precision can only be achieved by accounting for the horizontal displacement of the node as it moves between depths over a finite duration, owing to its profiling velocity being finite. Hence the finite float velocity must be considered. By taking into careful consideration the forces acting on the profiler, formulae for the maximum float velocities and electrical energy used in profiling were derived in Appendix B.

The float node movement scheme we proposed for extending coverage in 3D UWSNs was presented in Chapter 5 . The 3D coverage problem is formulated as a repeated trajectory planning task. Each float is assigned a position to frequent, out of a set of points distributed throughout and which together fully cover the RoI.

The performance of the scheme in a pollution monitoring scenario was analysed by simulations in Chapter 6 . A common pollution monitoring scenario, Lagrangian tracking, is evaluated, where the UWSN needs to simultaneously track and sample [43] an advecting pollution patch (for instance an oil spill or HAB). UWSNs are simulated in our realistic depth-decaying 3D coastal current mobility model, Current 2. This ocean environment is particularly amenable to float-based Lagrangian tracking. Our node movement scheme and in general all float-based path planning strategies only 
work well when the currents at each horizontal position point in many different directions along the depth profile. It is typical (such is the case in boundary currents and rivers for instance) and is the case in Current 2 that the current only points in one direction in the world frame. However, current speed generally decreases with depth. Relative to a drifting feature, floats will often be able to move ahead and lag behind it by ascending or descending. This creates the necessary conditions for 1D controllability, if not $2 \mathrm{D}$ and $3 \mathrm{D}$ controllability when random velocity components from inertial currents are factored in. We showed that the moving RoI tracking problem can be mapped to an equivalent static RoI monitoring application after applying a suitable frame transformation, which we derived in Appendix B.

The simulation results showed that while full coverage at all times can be achieved by AUVs but not by floats, similar levels of partial coverage obtained by UWSNs consisting only of AUVs can be met by float-based UWSNs that cost less to deploy. Our work thus provides a recommendation for hybrid UWSNs consisting of many types of devices, with inexpensive drifting nodes and profiling floats forming the bulk of the sensors. The floats can use the currents and their vertical mobility to cover most of the RoI. However, there may be regions where the water flow makes it difficult for floats to access, for example because the currents diverge from there. For this reason, the network is alo accompanied by a number of AUVs that move to plug coverage holes not easily or not immediately reachable by floats.

In determining the lifetime of the network, we find that both energy and dispersion limit the lifetime of the network. Without propulsion, the nodes are carried out of and cease to cover the monitoring region. Using mobility to remain with the region, at the end of a 5 day mission duration floats have had to use up some or almost all of their battery capacity in profiling.

A major emphasis in the design of the scheme was ensuring its implementability. The scheme can be immediately deployed as described on existing ALACE float platforms and using ADCPs for sensing. Owing to the low-bandwidth and unreliable nature of present-day underwater acoustic communications and the inaccuracy of current forecasts [181], a communications-free approach was taken where nodes rely on velocity measurements made by themselves in decision-making.

The scheme is intended as an interim solution until more data on ocean currents has been collected. The contemporary situation with regards to UWSN research resembles a catch-22 problem. As discussed in Section 2.2, past methods for sensing underwater currents are limited. This has resulted in a lack of current mobility models, and numerical ocean system outputs with low spatiotemporal resolution that 
are relatively accurate only for the water near the surface [181]. These systems are initialized mostly with ocean surface data, which can be readily obtained from satellite and HF radar scans. For the deep water state of the ocean, sparse historical data on the vertical current profile is employed [101]. Drifting UWSNs have been proposed to sense subsurface currents [88]. However, the performance of drifting UWSNs and the proper evaluation of protocol schemes, in order to determine which will work in practise, is dependent on knowing beforehand the currents in the deployment region.

We recommend the rapid real-life deployment of networks implementing interim schemes such as ours. These networks can be employed to measure the currents, where networks under such control have been shown (for example, in the simulation results of our scheme) to tend to be more efficient at remaining more clustered together covering the RoI than networks performing no mobility at all or some AUV only solutions. These UWSNs thus are better able to provide high resolution current velocity data, which is needed to adequately model the short-range neighbour mobility interactions in UWSNs and small-scale current processes at present lacking in numerical ocean models [37]. With this data, ocean forecast models can be made more accurate, enabling node movement schemes that exploit velocity predictions.

\subsection{Future work}

This section identifies several ideas that could be used to extend our work.

In Sections 3.6-3.7, we identified deficiencies in MCM that are carried over in our 3D extensions of the model. The flow being described by MCM is primarily geostrophic, when it is intended to represent a coastal current. Tidal and inertial current components make up a major proportion of the overall velocity in these currents. For the model to more accurately reproduce the velocity field inside a coastal current, these high-frequency effects should be represented, and several existing approaches that could be used in future to incorporate these high-frequency effects into the model were highlighted.

The current models proposed in this thesis have only been able to address the lack of realism in mobility models for two flow types, coastal currents and western boundary currents, of which the Gulf Stream is one. Deployment sites for UWSNs encompass many regions, from bays, estuaries, rivers and lakes to the open ocean, each having distinct current patterns. For example, in rivers we would expect the flow to be strongly directed downstream [109] while measurements of the currents in lakes suggest that fluid parcels circle the lake [120]. Network protocols and node 
movement strategies will likely perform differently in separate currents. Given the wide differences in currents across ocean regions, some works have considered it pointless to use realistic current models [119]. The performance of a node movement scheme in regions outside of the zone for which the current model is defined will not be reflected by its evaluated performance in the model. hence, it might be better to demonstrate that the strategy works in simple, stochastic, random walk models, which are unrepresentative of these other regions but are more relatable to the WSN community.

We propose instead that in future UWSN protocols be evaluated in simulation for not one current model, which can be criticized for the preceding reasons, but for a broad range of models representing different flow types. Researchers can then get a clearer picture of the deployment areas in which schemes are likely to perform well. Development of realistic 3D underwater current mobility models representative of regions throughout the ocean (one of the original thesis goals) remains an ongoing research challenge. For instance Tan et al. [190] mention that equivalent models for deep sea environments currently do not exist. In the limited time available to this thesis, we have advanced the understanding of coastal and boundary currents.

The currents could be classified based on the underwater zone, with the scheme being evaluated for each zone by a model representative of it: an estuary, deep ocean, river, lake and so forth. However, the properties of the flow may vary within zones, between lakes for instance. A superior alternative may be to quantify currents based on features of the mobility. A database of zones throughout the world's oceans can then be mapped to this metric. Current patterns at even intervals throughout this space can then be selected as advecting fields used to simulate the scheme's performance. The likely performance of the UWSN in different regions can then be estimated based on the simulated performance, and the nearness in this space of the current in the candidate deployment zone to the current where this performance was obtained. In meteorology, flow structures are assessed based on elemental quantities $\zeta$, $\delta$ and $d$, representing pure rotation (vorticity), divergence and deformation [78]. The velocity fields of flows characterized by varying levels of these elemental quantities are pictured in Figure 7.1. This could serve as a basis for how current velocity fields are quantified, with additional terms to evaluate how time-varying the flow is. A complication is that over the duration of a mission, a UWSN may experience many different current types.

The prototype node movement scheme we proposed had a number of limitations. Primarily, the strategy only allowed nodes to use current data that they themselves 


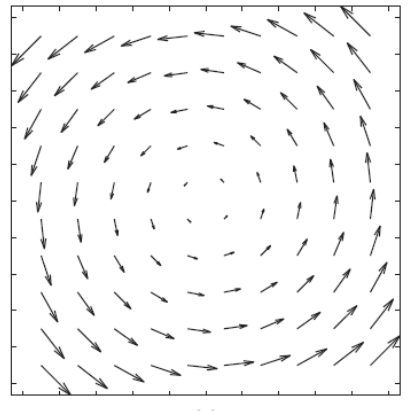

(a)

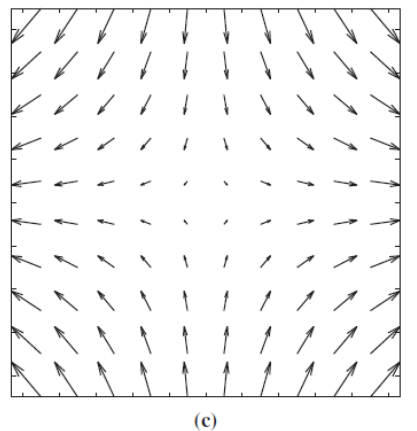

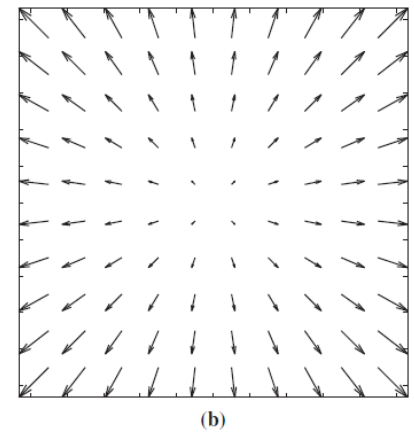

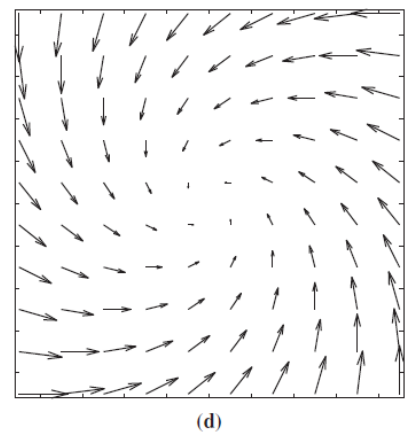

Figure 7.1: Velocity fields associated with flows exhibiting a) pure vorticity, b) pure divergence, c) pure deformation, and d) a mixture of vorticity and convergence. Image from [78].

could measure utilizing an ADCP, deeming present-day ocean predictions too inaccurate to be of value. The performance of this scheme thus does not answer the question, "what coverage performance could be achieved in a float-based UWSN, if ocean currents could be predicted and the network had global knowledge of them to plan the movement of nodes around?"

A possible approach that takes advantage of current predictions is sketched. It is likely that underwater acoustic communications will remain low-bandwidth, being physically constrained by such properties of the underwater acoustic channel as attenuation that increases with frequency [151]. A network scheme that disseminates global ocean prediction data to each node while they are underwater will create congestion and incur considerable overhead. Thus, a strategy where the predicted ocean state at a centralized surface sink location is studied. The sink can download the data from the land-based supercomputer [96] where the model is being run via medium-bandwidth radio communications. As nodes come to the surface, they can request their next trajectory, terminating with the float surfacing again, from the centralized controller via one-hop RF communications. The sink generates a trajectory, which it also records so that it is aware of the future predicted positions of all nodes as well. 
We observe that nodes will tend to, or can be scheduled to, surface asynchronously. In the scenario in Section 6.1, up to 80 nodes were deployed in the UWSN and the most rapidly they could surface again was set to 3 hours (the minimum time to cycle between the surface and bottom of the RoI at $500 \mathrm{~m}$ depth and $0.2 \mathrm{~ms}^{-1}$ speed being 1.4 hours). On average, across the entire network a node will surface once every two minutes. Within this time the controller should be able to generate a trajectory for the node, by performing a local search [135] on a guess solution, and provide it to the node in time for the next float to surface.

This suggests a way to improve the performance of Scheme 1 that we proposed using current predictions. In Scheme 1, nodes repeatedly move to 3D points distributed throughout the RoI in the hopes of fully covering it. A rapid method, based on the solution of a convex optimization problem, for positioning floats onto the point was developed. The generated trajectory would only place the float exactly on the goal if the currents were positively spanning and only slowly varied spatially and temporally. Real currents will be time- and spatially-varying, resulting in the float ending up only in a neighbourhood of its target needed for coverage. In this new case, with future currents known, the centralized controller can predict via numerical integration the actual trajectory of the float. The sink can iterate over the velocities the node is planning to move at, possibly finding a trajectory that positions the node closer to its goal. The assumption is that the solution found via the convex optimization problem that brings the float close to its target position, is also near to an exact solution in the space of all possible trajectories. This solution can then be rapidly found utilizing a local search algorithm. Using these trajectories, the scheme will more closely approach its ideal performance, which envisioned all nodes being able to move exactly onto the high-level goals set by the strategy.

The iterated trajectories can also address another issue with Scheme 1. Full coverage is achieved in the strategy when multiple nodes are assigned to each goal, and each float arrives at its target as another devices leaves, so as to fill the coverage hole left by the other profiler. The original scheme relies on random arrival times to eventually achieve this when sufficient numbers of nodes are deployed. The centralized controller in the extended strategy has full knowledge of the future locations of other nodes in the network. The fitness with which iterated trajectories are compared to the original trajectory can thus incorporate how many sections of the RoI that would have been uncovered would be visited by the node. The controller can then guide floats to reach its goal when other nodes are not present, as well as move through other coverage holes during the rest of its trajectory. 
A test scheme was implemented by the author employing simulated annealing to perform a local search on the guess trajectory. However, no noticeable improvement in the coverage metric was observed, with the local search algorithm not causing the fitness of the trajectory to significantly change. The results have not been reported in this thesis because of space, and due to concerns that it may have not been a failing of the core concept but the inefficiency of our implementation. The simulated annealing-based search that was implemented in MATLAB could only manage 40 iterations in two minutes processing time on our workstation. With sufficient optimization, global searches have been able to be performed in similar time in [108]. Further investigation is warranted. 


\section{Appendix A}

\section{The physical origins of ocean}

\section{currents}

Ocean currents arise from tidal, wave and wind processes [136] that occur across widely varying temporal and spatial scales. The currents resulting from each process superpose to give the instantaneous current velocity measured at a point [158]. This appendix reviews the main component of the current velocity and explains how these components combine in the mean current.

\section{A.1 Tidal and wave-induced currents}

The varying gravitational pull of the Moon and Sun on the Earth causes the level of the sea at shorelines to rise and fall. High and low tide are accompanied by horizontal currents directed towards and away from shore respectively [136]. There are known as the flood and ebb current. As a simplified explanation, a flood current is needed to supply the water that causes the rise in water level. An ebb current removes the water at low tide, lowering the water level.

When waves strike the shore at an angle, not all of their energy is absorbed by the land but some is imparted to the surrounding water. This leads to the formation of longshore currents directly next to and running parallel to the shore [136]

\section{A.2 Wind stress-induced currents}

The wind stress on the surface of the ocean results in inertial oscillations from the changing strength of the wind [56] and Ekman currents arising from a steady 
wind [140]. The wind exerts a force in the direction of the wind on the sea surface. The distribution of the force is expressed by the wind stress, $\tau_{\text {wind }}$. The wind stress is empirically related to the wind velocity by [185]

$$
\tau_{\text {wind }}=F / A=\rho_{\text {air }} C_{\mathrm{D}} U_{10}^{2}
$$

where $\rho_{\text {air }}$ is the density of air, $C_{\mathrm{D}}$ is the dimensionless drag coefficient and $U_{10}$ is the wind speed at $10 \mathrm{~m}$ above the sea.

The force gives rise to motion. The momentum of the surface layer is then transmitted downwards by fluid friction [140], stemming from the exchange of individual water molecules (molecular viscosity) and macroscopic parcels of water (eddy viscosity). As particles displaced from higher up collide with molecules in the lower layer, they impart some of their momentum, on average accelerating the lower layer in the direction of the surface stratum.

If the wind was the only force acting on the ocean, the resulting current would be in the same direction as the wind. The ocean is also affected by an apparent Coriolis force, however. The wind stress is described in a local Cartesian system of co-ordinates, where a point is considered fixed relative to the Earth, and the dynamics of the system are given by Newton's laws of motion. These apply only to a non-accelerating or inertial frame of reference. The Earth is in fact accelerating, rotating from the west towards east. This means that water moving northwards or southwards of the Equator will be seen to deflect to the right or left respectively (in the conventional co-ordinate system, where $x$ is positive toward east). This is because the Earth has rotated westwards under it in the meantime while, being a fluid not firmly connected to the ground and being rotated with it, maintains its northward or southward course in an inertial frame. Newton's laws of motion clearly require careful application for such a system. For instance, Newton's first law of motion states that if the net force on an object is zero, then its velocity is constant, whereas the direction that the northwards moving body is proceeding at is changing.

To account for the Earth's rotation and be able to describe the motion of objects in the region frame of reference by Newton's laws, a fictitious Coriolis force must be added in. The force affects all moving bodies and acts $90^{\circ}$ cum sole (with the sun: to the right in the Northern Hemisphere, to the left in the Southern) of the direction of the body's motion [140].

The interaction between the wind stress and Coriolis effect impacts the direction of the current. When a steady wind is blowing, the steady-state current component that is produced must be at an angle for the wind-induced and Coriolis force to 
balance out. The resulting Ekman current is shown in Figure A.1. The current at the surface is directed $45^{\circ}$ from the wind, and increases in angle with depth. Features of the Ekman current have been commonly observed throughout the ocean, where Price [152] finds that the current they measured can be adequately represented by an extended Ekman model.

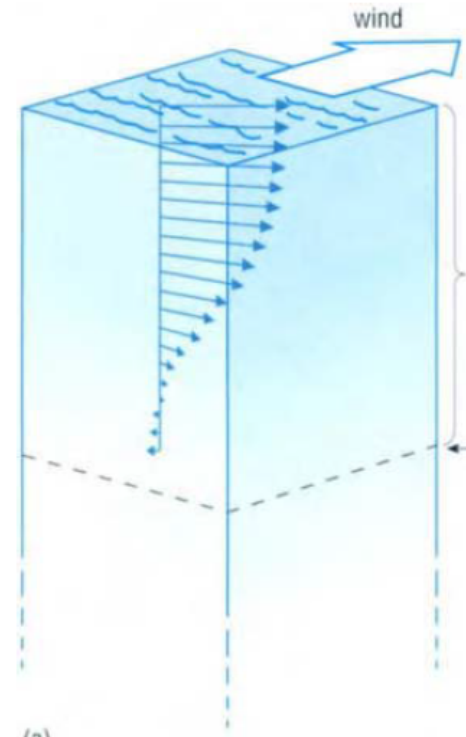

(a)

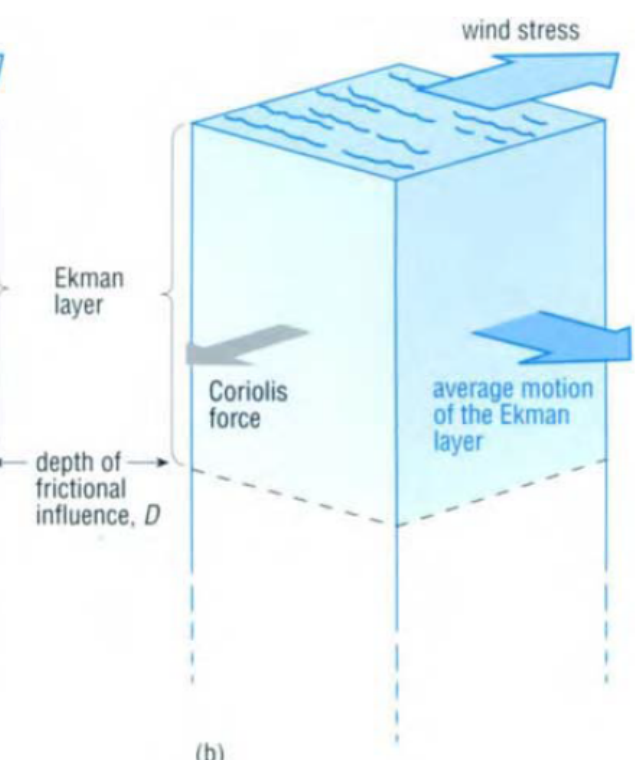

(b)

Figure A.1: Theoretical Ekman current derived by consideration of the impact of viscosity and the Coriolis effect on an ideal ocean. Image from [140]. The current is directed away and deviates further from the wind direction with depth, decreases in speed with depth and (b) the Coriolis and wind forces balance each other out.

Due to the momentum of the water, it takes some time for the water to accelerate to the steady-state Ekman state and may never converge if the wind varies rapidly. The difference in the wind stress-induced component of the current between the expected Ekman pattern for a measured wind stress, is labelled the inertial oscillation [40].

The depth to which wind stress-induced currents extend to is limited. Fluid parcels and molecules can only move freely between layers in the upper portion of the ocean. In this mixed layer, churn set up by waves whipped up by the winds causes the fluid to be homogenized with roughly uniform properties such as temperature. Below this layer, the ocean is strongly stratified by density. The density of water increases with pressure and decreasing temperature, resulting in greater densities at depth. This acts to suppress the vertical mixing needed to transfer momentum across layers [140], which also results in the vertical speed of currents being negligible [32]. 
A large external disturbance is needed to displace fluid parcels from the level they are occupying, and even then the fluid parcels experience a restoring net buoyancy force that quickly returns them to their original depth before much diffusion or collisions can take place. The depth of the mixed layer is roughly $10-200 \mathrm{~m}$ and depends on the strength of the winds [186]. In particularly calm waters such as the Sargasso Sea, the depth of the layer is around $25 \mathrm{~m}$ [152]. Measurements have found the wind-generated acceleration below the mixed layer to be near zero [152].

The speed of Ekman currents decreases steadily with depth as only a fraction of the kinetic energy of each layer is transferred to the layer below it [170]. Within the fluid, individual molecules are moving in random directions. The velocity of each molecule is the sum of a random and a constant component, with the latter equal to the macroscopic velocity of the fluid. The mean component to the velocity of parcels means that collisions cause parcels to tend on average to move further in the direction of the wind, which is observed as a force along that vector. The stochastic component results in bodies striking each other at odd angles and causes the particles to careen off in random directions. Rather than adding to the overall directed motion, some of the energy is lost in increasing the energy of the random molecular motion of the fluid, otherwise known as heat, as it is transmitted downwards [64].

The resistance of the water to wind stress is modelled by a vertical eddy viscosity term, $A_{\mathrm{z}}$, where the term is related to the components of the wind stress in the $x$ and $y$-directions, $T_{\mathrm{xz}}$ and $T_{\mathrm{yz}}$, water density $\rho$ and the velocity shear by [185]

$$
\begin{aligned}
& T_{\mathrm{xz}}=\rho A_{\mathrm{z}} \frac{\partial u}{\partial z} \\
& T_{\mathrm{yz}}=\rho A_{\mathrm{z}} \frac{\partial v}{\partial z}
\end{aligned}
$$

By considering the velocity of the fluid that results in the Coriolis force balancing out the force of the stress applied to an infinitesimal box element of water, having been propagated down by a constant stress on the surface and hence constant wind, the steady-state Ekman current arising from the blowing of a steady wind can be derived as

$$
\begin{aligned}
& u(z)=V_{0} \exp (a z) \cos (\pi / 4+a z) \\
& v(z)=V_{0} \exp (a z) \sin (\pi / 4+a z)
\end{aligned}
$$

where $V_{0}$ and $a$ are constants. For a wind blowing the north, $T_{\mathrm{xz}}=0, V_{0}=\frac{T_{\mathrm{yz}}}{\sqrt{\rho^{2} f A_{\mathrm{z}}}}$ and $a=\sqrt{\frac{f}{2 A_{\mathrm{z}}}}, f$ being the Coriolis parameter. A full derivation can be found in Stewart et al [185]. As can be seen from the equation, the direction of the current 
rotates with depth as a function of cos and decreases in magnitude as a function of $\exp (a z)$.

\section{A.3 Geostrophic currents}

The wind not only causes currents due to the direct action of local winds. Persistent, large-scale wind systems also leads to water being pushed and "piling up" in areas [140]. As a result, the oceans are not flat but at different heights. The sloping ocean surface gives rise to horizontal pressure gradients that, when balanced with the Coriolis force, results in geostrophic currents [185].

The forces set up by uneven sea surface heights are illustrated, in Figure A.2, in a simple scenario, where the water density, $\rho$, is treated as constant. The pressure at depth $z$ is due to the weight of the overlying water and is given by the hydrostatic equation [140]

$$
\begin{aligned}
p(z) & =p_{0}+\int_{z}^{0} \rho(z) g d z \\
& \approx p_{0}-\rho g z
\end{aligned}
$$

Consequently, the pressure at two points at the same level horizontally will be higher at the point that is further to the right, with $\rho g(z+\Delta z)>\rho g z$, where $z$ is the depth below the water's surface at the leftmost point and $\Delta z$ is the height the sea surface at the rightmost point is raised above the sea surface at the leftmost point.

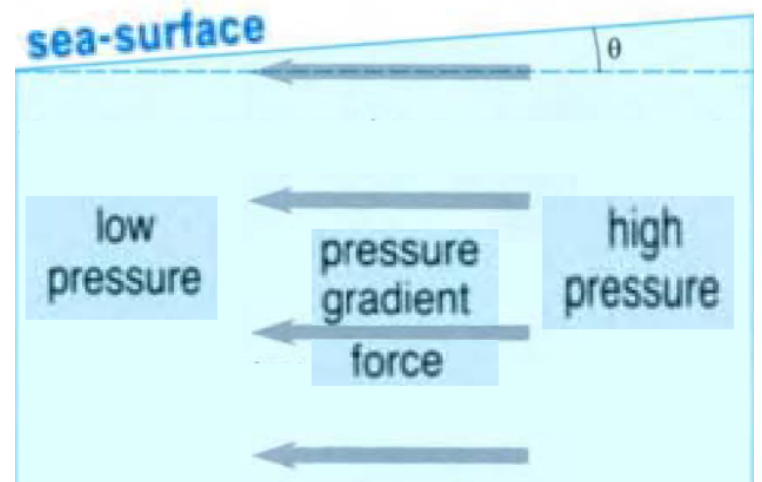

(a) CROSS-SECTION

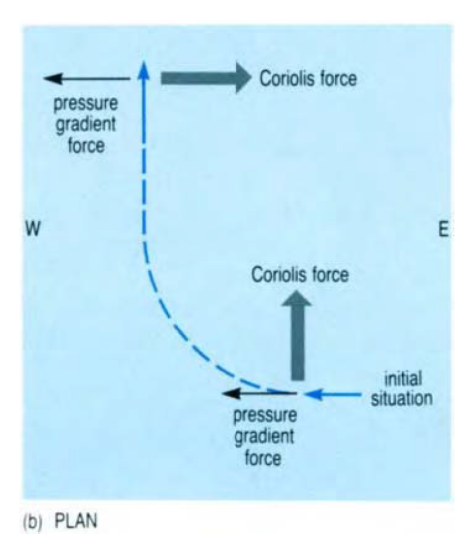

(b)

Figure A.2: a) Cross-section view of a sloping sea surface, giving rise to a horizontal pressure gradient force, and b) the geostrophic current that results, viewed from above. Images from [140]. 
Consider the forces affecting a fluid parcel, which may be viewed as a small box at depth $z$. The pressure on the left side of the box exerts a rightward force, $F=p A$. Likewise, a leftward force is created by pressure on the right side of the box. As the pressure on the right side of the box is greater, this creates an unbalanced horizontal pressure gradient force acting to the left given by

$$
\begin{aligned}
F & =\left(p_{2}-p_{1}\right) A \\
& =\left(\left(p_{c}+\frac{d p}{d x} \Delta x\right)-p_{c}\right)(\Delta x)^{2} \\
& =\frac{d p}{d x}(\Delta x)^{3}
\end{aligned}
$$

where $\Delta x$ is the length of each side of the box. Initially, this force causes the fluid to move in the direction of the pressure gradient force. However, as the motion is affected by the Coriolis force, the flow stabilizes into a steady-state geostrophic current aligned $90^{\circ}$ from both the Coriolis and pressure gradient force.

\section{A.4 A depth-decaying mean current}

The current components described vary along different time-scales. Tidal currents having a semi-diurnal or diurnal period. The inertial currents and motion due to waves depend on the immediate value of the wind and sea state, making them essentially impossible to predict far into the future and so they are typically modelled stochastically [56]. Observational data has found that these high-frequency components have a zero mean. When the mean current over a day or more is calculated, these terms disappear in the ensemble average of the mean current, leaving the contribution of only long-term processes, geostrophic and Ekman currents. The ensemble average then is equal to the sum of the geostrophic and Ekman current caused by the averaged or steady component of the wind stress measured at each day [152]. This means that typically the underlying current is depth-decaying, as both Ekman and geostrophic currents are known to decrease in strength with depth. 


\section{Appendix B}

\section{Controllability analysis of Current 2 for floats}

Many float motion planning strategies [93, 130, 182], as surveyed in Section 5.1.2, are predicated on the currents everywhere positively spanning the $2 \mathrm{D}$ plane. This positively spanning property is the necessary and sufficient condition, established in the controllability criterion (Corollary 5.1.2.1) derived by Smith and Dunbabin [181], for a float with infinite vertical velocity to be globally controllable in $\mathbb{R}^{2}$. That is, from any point in $\mathbb{R}^{2}$, the profiler can reach another point in $\mathbb{R}^{2}$ in finite time [39]. The node movement scheme that we have proposed in Chapter 5, Scheme 1, also relies on such current conditions to function.

This assumption is usually justifiable as analysis of numerical ocean models has showed that these current conditions are predicted to occur in many regions of the ocean $[130,181]$. In this appendix, we investigate how well the positively-spanning property applies to the physically-inspired 3D depth-varying coastal current, Current 2 (Table 3.6), we developed. This is assessed by the use of controllability charts after Smith and Dunbabin [181]. A top-down graph of the RoI is produced. The current velocity at regularly spaced grid points is checked to see if they positively span the $2 \mathrm{D}$ plane. If so, the point is coloured red rather than blue. The degree with which the positively spanning assumption holds is quantified by the proportion of the chart that is red.

The current velocity field in the static frame of reference is not the only one evaluated for controllability. Although one application for UWSNs is area coverage of fixed areas such as oil and gas fields, shipwrecks (salvage sites) and harbour entrances [41], another important use for UWSNs is Lagrangian tracking [43]. Not all underwater features are stationary. Pollution features in particular, such as 
harmful algal blooms and patches of low-oxygen water corresponding to chemical spills, drift with the surrounding water. The evolution of these phenomena are best studied with a sensor network that moves with the feature. We show in this appendix that the problem of keeping floats in a RoI drifting with the feature can be equated to controllability of floats in a transformed current velocity field, given by the flow velocities observed in a frame of reference moving with the drifting RoI. This transformed current velocity field is also assessed for controllability.

It is found that it would be impossible for a float network to remain covering a static RoI in this current. In a frame of reference moving with a drifting underwater point, floats are observed to be generally controllable. Our Scheme 1 is thus likely to perform as intended in providing 3D monitoring of a region around an advecting feature.

This appendix is organized as follows. The presence of the positively spanning property in Current 2 is evaluated in Section B.1 via the use of controllability maps. Section B.2 introduces and formally defines the Lagrangian tracking application. The ability for floats to be able to keep track of a Lagrangian drifting RoI is shown in Section B.3 to be equivalent to its ability to move within a transformed current velocity field. Finally, Section B.4 assesses the controllability of floats in the Lagrangian tracking drifting frame of reference.

\section{B.1 Controllability analysis of Current 2 in the static frame of reference}

We generated controllability maps for Current 2, where the current velocity in relation to a local Cartesian coordinate system fixed with respect to the rotating Earth is given by(3.17). The region is divided up into a grid in $2 \mathrm{D}$. At each grid point the current velocity is evaluated at heights spaced $25 \mathrm{~m}$ apart from $0 \mathrm{~m}$ to $-500 \mathrm{~m}$, a typical depth range used in UWSN simulations [205]. The grid point is marked red if these velocities positively span the horizontal plane, otherwise blue.

Positively spanning is determined using the angle criterion (5.5). This states that the set of vectors positively spans the $2 \mathrm{D}$ plane if and only if the maximum angle between adjacent velocities (when the velocity vectors are collected at a common point) is less than $\pi$. To relate how the current along the depth profile combine to meet or fall short of this criterion, the velocities at all 21 depths are arranged around each point. For each depth, a line segment is drawn from the point where the current 
was measured in the direction of the current velocity, where each segment is drawn with the same length.

Graphs are generated for an $8 \mathrm{~km} \times 8 \mathrm{~km}$ rectangular area with one corner at the origin at times ranging from $t=0-12 \mathrm{~h}$ in Figures B.1-B.12. The $x$-axis and $y$-axis give the co-ordinates in the static reference frame. The purpose of this figure is to give a synoptic view of the controllability of the current that captures an entire meander, of wavelength $7500 \mathrm{~m}$. The current at grid points spaced $250 \mathrm{~m}$ apart are analysed. For reference, the current velocities in the same area at depths 0,250 and $500 \mathrm{~m}$ are plotted alongside. The current velocity vectors are colored and directly proportionately scaled in length according to their magnitude.

Additional figures focusing on and giving a higher resolution view of the different current regimes, described in Figure 3.5, are also produced, in order to fully characterize the current's suitability for float operations. The current in the static frame is evaluated in a $500 \mathrm{~km} \times 500 \mathrm{~km}$ axis-aligned box around a point on the centre of the jet, a separatrix and at the centre of a vortex at $t=0 \mathrm{~h}$ and $250 \mathrm{~m}$ depth. In static frame co-ordinates, the centre of each region are located at $\left[\begin{array}{ccc}3750 & 0 & -250\end{array}\right]^{T}$, $\left[\begin{array}{lll}3225 & 42 & -250\end{array}\right]^{T}$ and $\left[\begin{array}{lll}1875 & 152 & -250\end{array}\right]^{T}$ respectively. The position of these regions with respect to the static frame are displayed in Figure B.13 along with the streamlines of the current at $250 \mathrm{~m}$ depth in the comoving frame with coincident co-ordinates at $t=0 \mathrm{~h}$. A box located on a separatrix is surveyed so that the intermediate current in between the regimes can be investigated. The controllability maps of the jet regime are shown in Figures B.14-B.17, in Figures B.22-B.25 for the separatrix regime and Figures B.30-B.33 for the vortex regime. The controllability maps are displayed alongside the current velocity field within the region.

The process employed to determine the positions of these plots is described. The centre of the jet in the static frame is given by (see Section 3.5).

$$
y_{c}=B(t) \sin \left[k\left(x-c_{x} t\right)\right]
$$

The jet-centred box was arbitrarily determined to be placed at $x=3750 \mathrm{~m}$, half the meander wavelength. Consequently, the co-ordinates of the box centre are $\left[\begin{array}{lll}3750 & C(3750,0) & -250\end{array}\right]^{T}=\left[\begin{array}{lll}3750 & B(0) \sin (\pi) & -250\end{array}\right]^{T}=\left[\begin{array}{lll}3750 & 0 & -250\end{array}\right]^{T}$.

The vortex centre, $\left[\begin{array}{lll}1875 & 152 & -250\end{array}\right]^{T}$, is found as a location where the current velocity is zero in the comoving frame. The rationale for this is because the vortex consists of concentric closed streamlines in the comoving frame. The centre of the vortex is deemed to be where the streamline becomes vanishingly small, turning into 


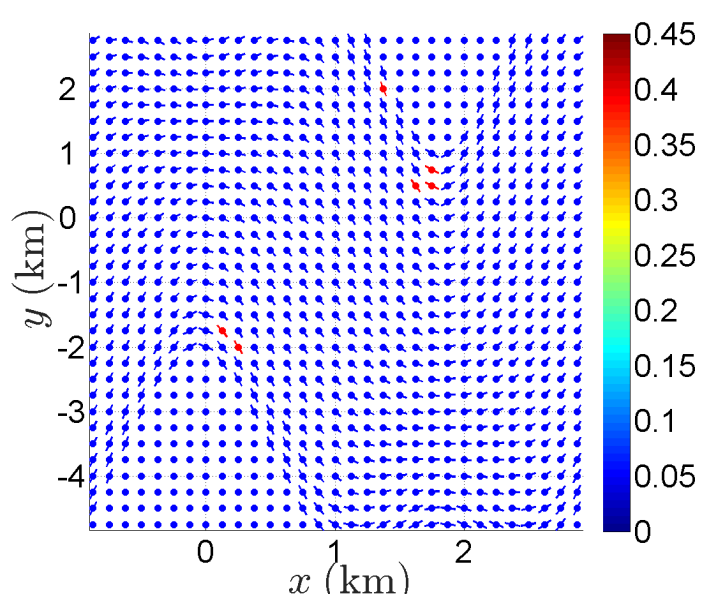

Figure B.1: Synoptic controllability map of Current 2 at $t=0 \mathrm{~h}$.

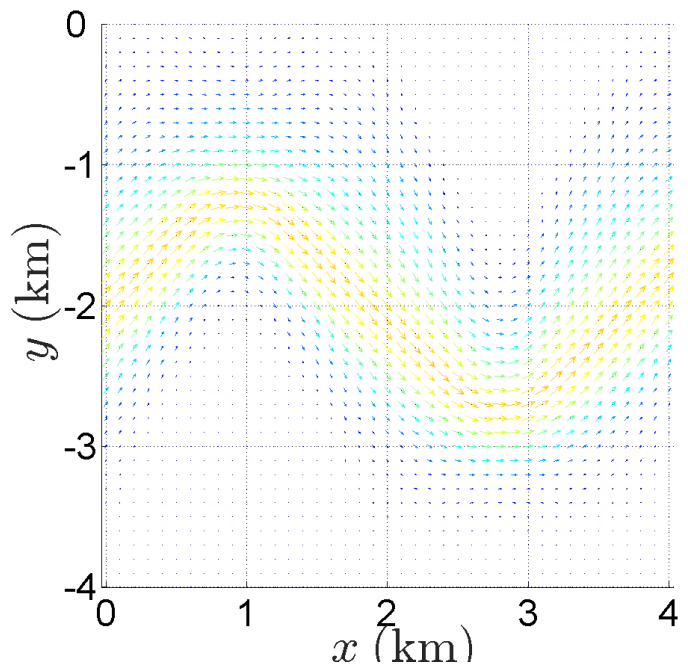

Figure B.3: Current velocity field at $t=0 \mathrm{~h}$ along $z=-250 \mathrm{~m}$.

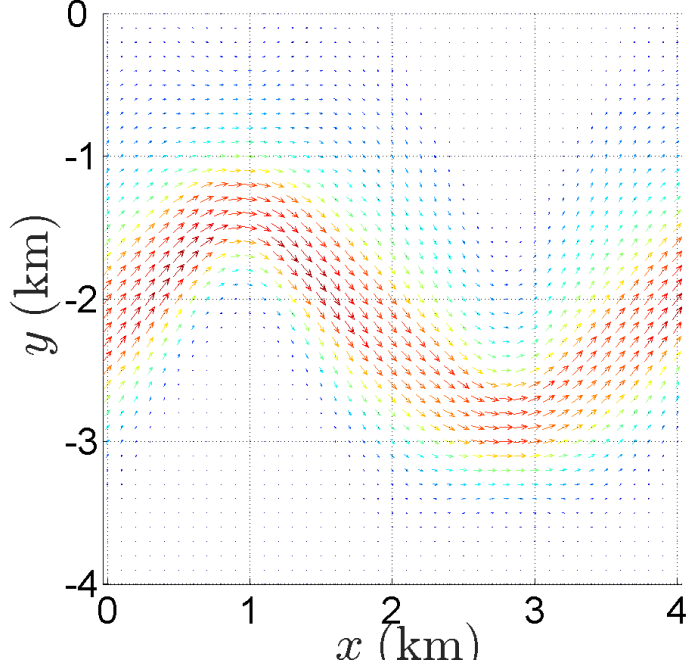

Figure B.2: Current velocity field at $t=0 \mathrm{~h}$ along $z=0 \mathrm{~m}$.

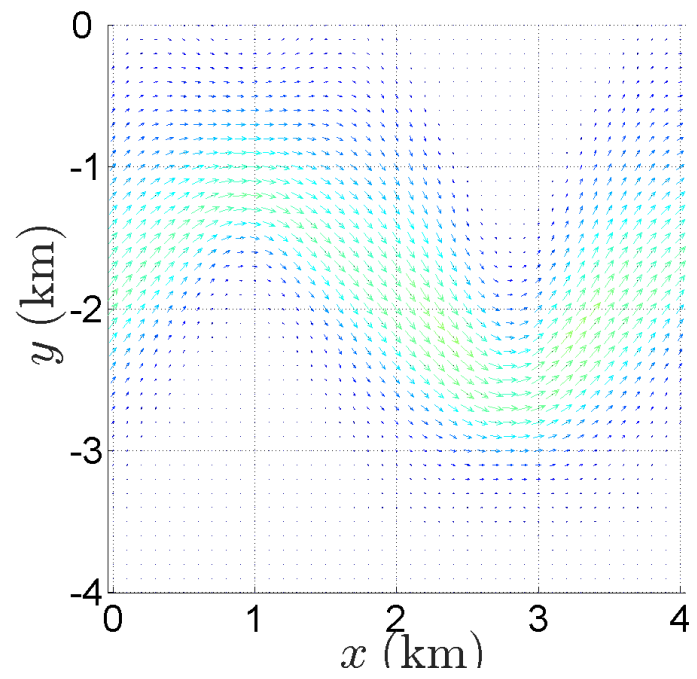

Figure B.4: Current velocity field at $t=0 \mathrm{~h}$ along $z=-500 \mathrm{~m}$.

a point. If the velocity field does not change, the streamline describes the path a massless fluid element would travel in the flow, and the speed of the field at this point must be zero. The position where the speed is zero is found numerically using MATLAB. MATLAB's fminsearch procedure is utilized, which given an initial estimate for the optimization variable employs a local simplex search method to find a point that minimizes the objective function. Co-ordinates within a vortex of $\left[\begin{array}{lll}1875 & 100 & -250\end{array}\right]^{T}$ are supplied as the starting point for the algorithm, where the magnitude of the current in the comoving frame is to be minimized. The position 


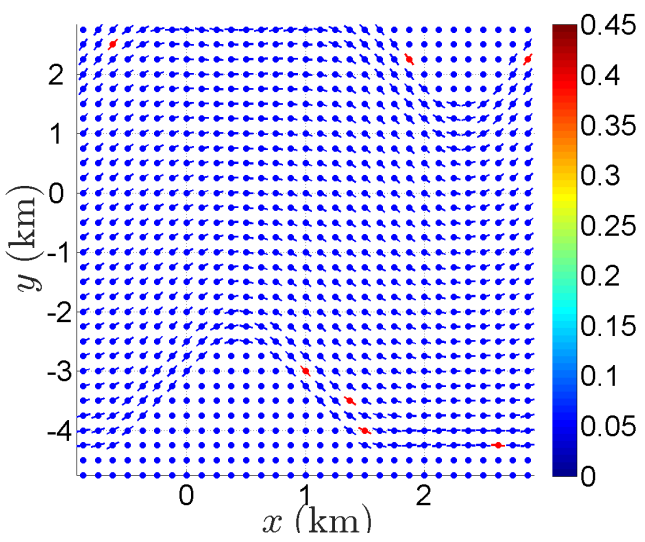

Figure B.5: Synoptic controllability map of Current 2 at $t=6 \mathrm{~h}$.

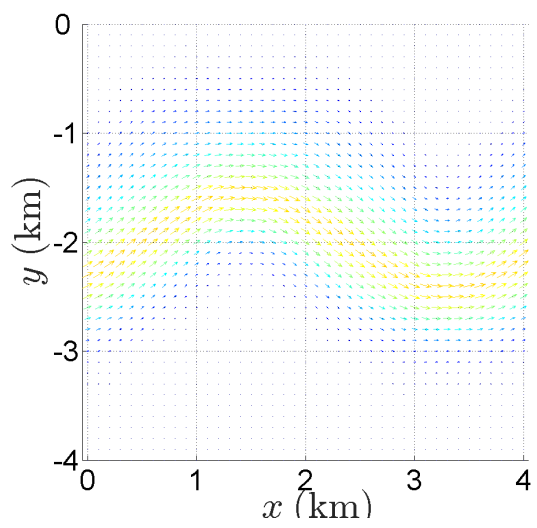

Figure B.7: Current velocity field at $t=6 \mathrm{~h}$ along $z=-250 \mathrm{~m}$.

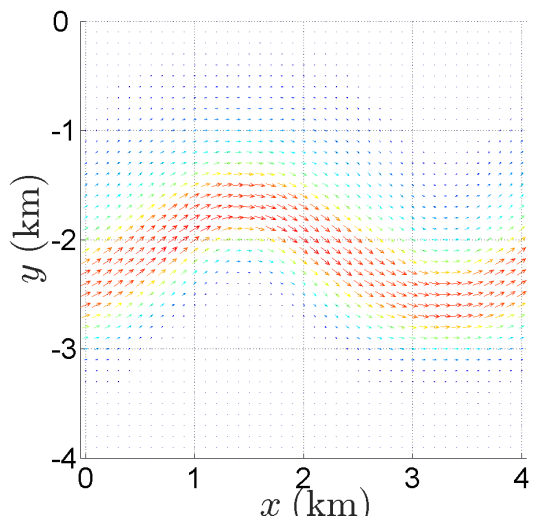

Figure B.6: Current velocity field at $t=6 \mathrm{~h}$ along $z=0 \mathrm{~m}$.

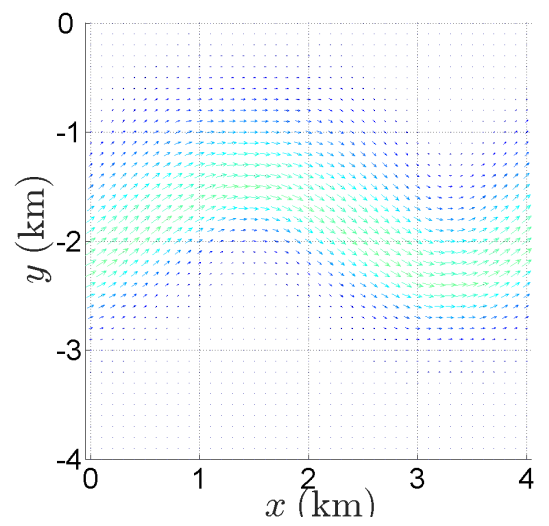

Figure B.8: Current velocity field at $t=6 \mathrm{~h}$ along $z=-500 \mathrm{~m}$.

found by the method is $\left[\begin{array}{llll}1875 & 152 & -250\end{array}\right]^{T}$. Visual inspection of the solution's location in the streamline pattern of Figure B.13 confirms that it is at the centre of a vortex.

The centre of the separatrix-centred RoI, $\left[\begin{array}{lll}3225 & 42 & -250\end{array}\right]^{T}$, is found by drawing a line between the centres of the jet and vortex that have been determined. The line is broken up into a number of fine intervals and traversed from the centre of the vortex, computing the streamline at each point using MATLAB's streamline function. The algorithm stops when it comes to a point where the streamline no longer loops, $\left[\begin{array}{lll}3225 & 42 & -250\end{array}\right]^{T}$, and this stopping location used as the centre of the box. It must lie on the separatrix as within the vortex regime, the streamlines are closed, whereas in the central jet, the streamline is a one-to-one function of $x$. The boundary between the regimes thus occurs when the streamlines an incremental distance in one direction from the line loops, and the streamlines an incremental 


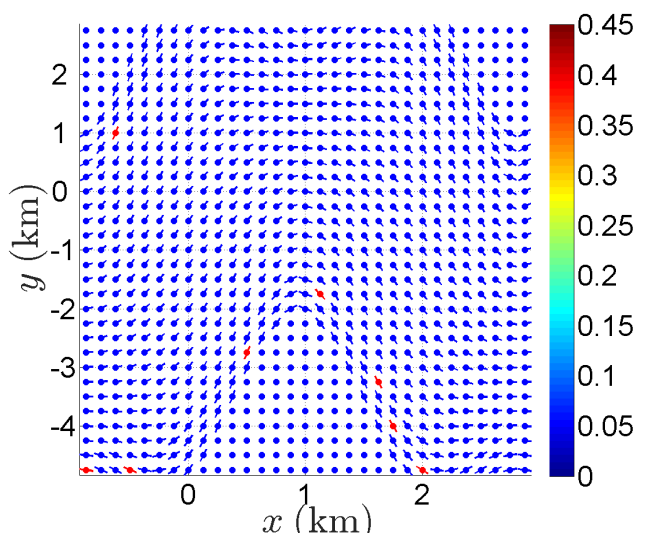

Figure B.9: Synoptic controllability map of Current 2 at $t=12 \mathrm{~h}$.

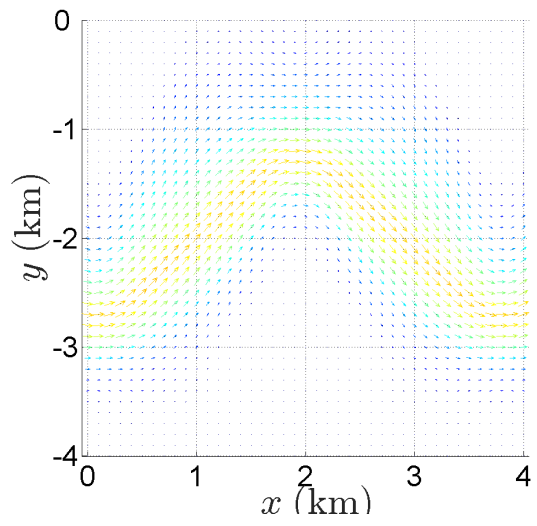

Figure B.11: Current velocity field at $t=0 \mathrm{~h}$ along $z=-250 \mathrm{~m}$.

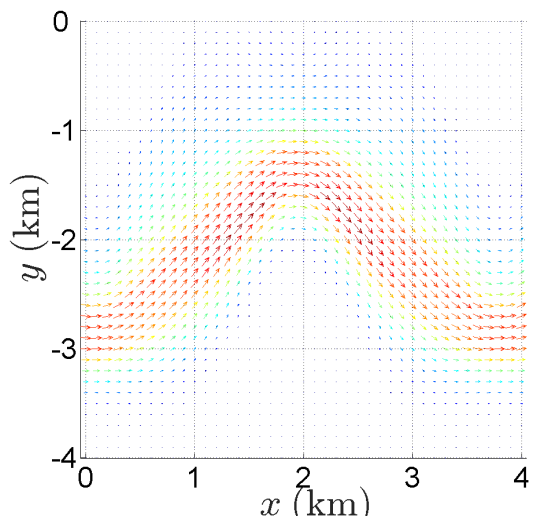

Figure B.10: Current velocity field at $t=0 \mathrm{~h}$ along $z=0 \mathrm{~m}$.

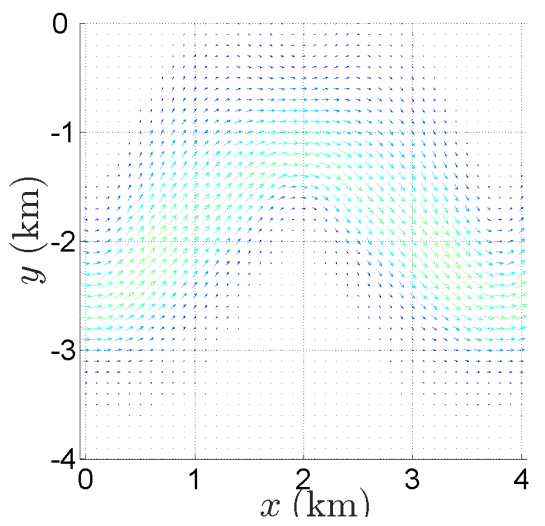

Figure B.12: Current velocity field at $t=0 \mathrm{~h}$ along $z=-500 \mathrm{~m}$.

distance in the other direction are one-to-one.

The controllability plots are now discussed. In each of the controllability maps, nowhere does the current positively span $\mathbb{R}^{2}$. The assumption that Scheme 1 relies upon is thus invalid. These results are expected. The current model employed defines a jet that, while meandering, is everywhere directed eastwards. Not just our scheme but any node movement strategy will be unable to use floats to cover a static RoI for a prolonged length of time, as all nodes will be eventually carried out eastwards of the RoI and there is nowhere a float can maneuver to, in order to find a westward current to reverse its motion.

It should be mentioned that this does not say that Scheme 1 is unlikely to work for static monitoring applications in all currents, only depth-decaying directed flows, such as the Gulf Stream and coastal currents, represented by Current 2, the model that was used. As shown by $[130,180]$, large parts of various ocean regions 


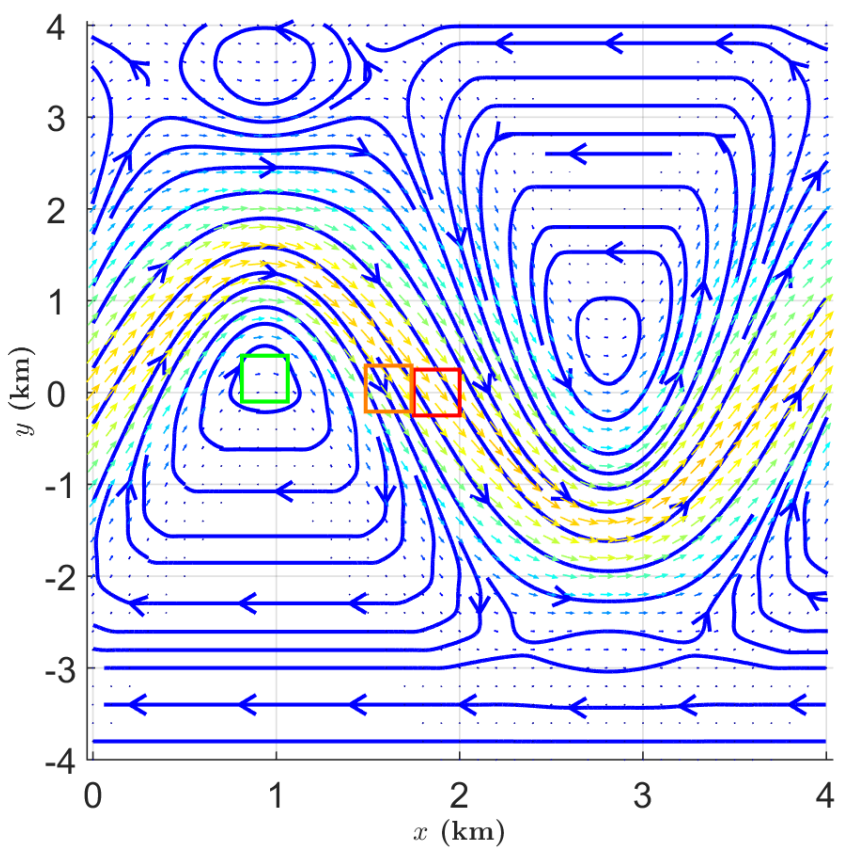

Figure B.13: Placement of the boxes in the world frame, set up to analyse the current in each regime, relative to the streamlines of Current 2 at $250 \mathrm{~m}$ depth and $t=0 \mathrm{~h}$ in the comoving frame of reference. The jet-centred box is marked red, separatrix-centred box orange and vortex-centred box green.

exhibit controllability properties that the scheme relies upon. The Humboldt current is another real-world example. Flowing off the coast of Chile, within $500 \mathrm{~m}$ of the surface the current positively spans the plane in $2 \mathrm{D}$ and with inevitably any horizontal variation $3 \mathrm{D}$, where the current flows predominantly northwards between $0-100 \mathrm{~m}$ depths, southwards between $100-400 \mathrm{~m}$ and northwards from $400-500 \mathrm{~m}$ depths [174].

\section{B.2 Lagrangian tracking of a drifting RoI}

Based on the controllability maps, floats cannot long remain covering a static RoI in the coastal current model that we have developed, Current 2. Similar results could also be expected of other highly directed flows such as in rivers or tidal currents. Often it does not make sense to monitor a fixed region, when the feature of interest is itself drifting at the speed of the surrounding currents, such as in algal bloom or pollution monitoring [43]. Then a network should move with the feature, covering an area encompassing the phenomenon, which is not just a point but forms an extended patch of algae or chemicals. By doing so the sensors can provide in-situ measurements 


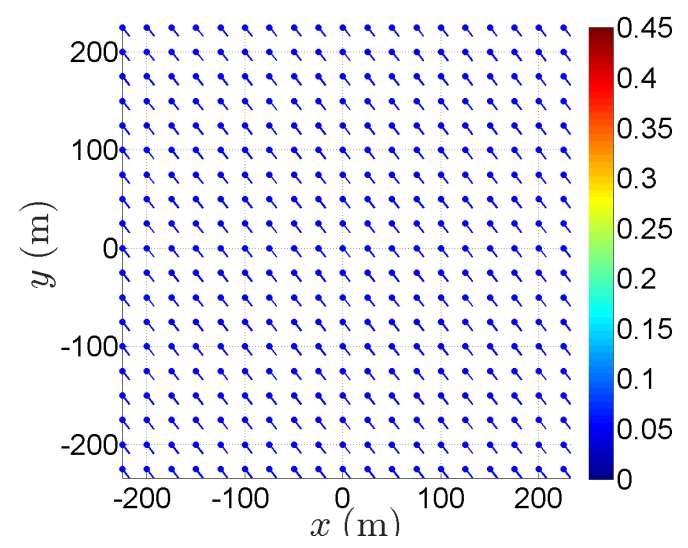

Figure B.14: Controllability map of the currents within the fixed, initially jet-centred, box at $t=0 \mathrm{~h}$.

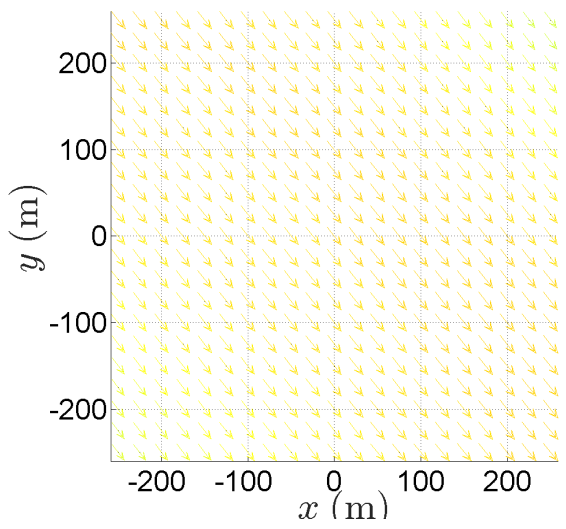

Figure B.16: Current velocity field at $t=0 \mathrm{~h}$ along $z=-250 \mathrm{~m}$.

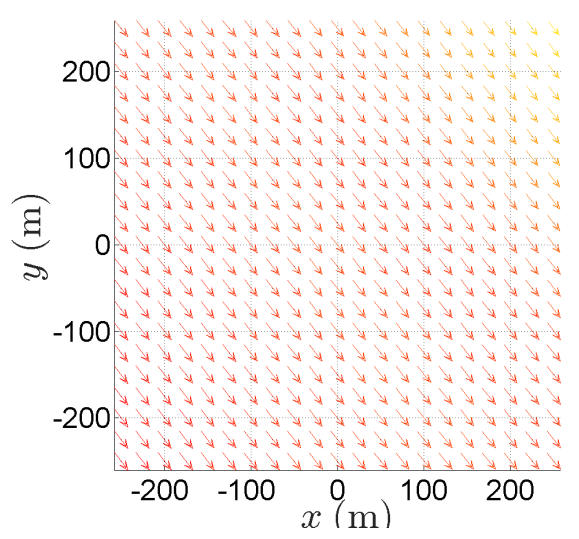

Figure B.15: Current velocity field at $t=0 \mathrm{~h}$ along $z=0 \mathrm{~m}$.

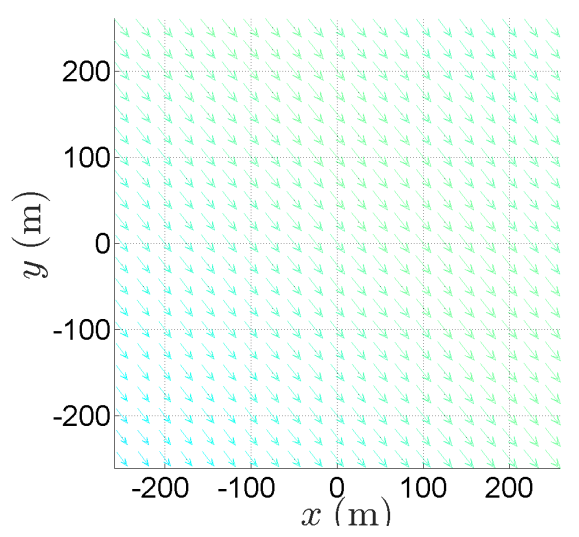

Figure B.17: Current velocity field at $t=0 \mathrm{~h}$ along $z=-500 \mathrm{~m}$.

throughout the feature, charting changes for instance in the composition of the bloom and pollution concentration over the lifetime of the feature as well as keeping track of its location. Moorings or floats deployed in a static RoI would only be able to briefly monitor the pollution before it leaves the area.

We formally define this Lagrangian tracking application, so-called because the location of the points to be monitored moves with the flow [44]. In this Lagrangian tracking application, we are interested in covering a fixed area around a drifting feature with a network of floats. The area may be defined as a rigid body with fixed shape and volume $L \subset \mathbb{R}^{3}[39]$. Attach a coordinate frame to the rigid body, as shown in Figure B.38. In stationary world frame co-ordinates, $\mathbf{p}=\left[\begin{array}{lll}p_{1} & p_{2} & p_{3}\end{array}\right]^{T} \in \mathbb{R}^{3}$ is the position of the body-fixed frame's origin. The feature is drifting with the currents, so the attached body-fixed frame's origin is thus defined as moving at the 


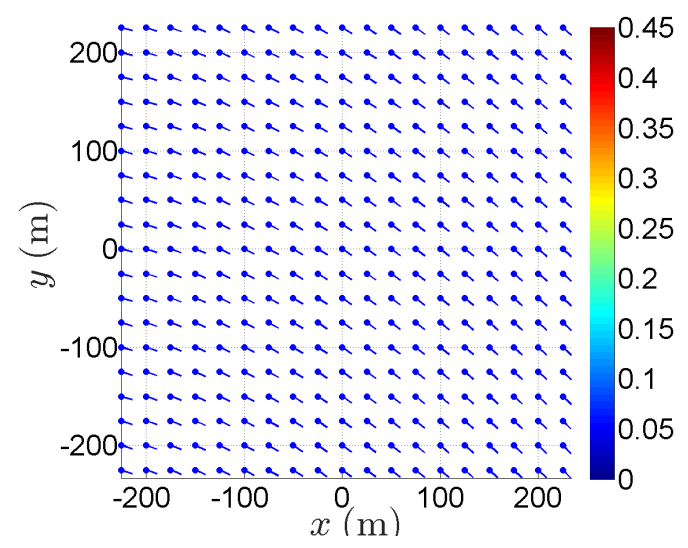

Figure B.18: Controllability map of the currents within the fixed, initially jet-centred, box at $t=9 \mathrm{~h}$.

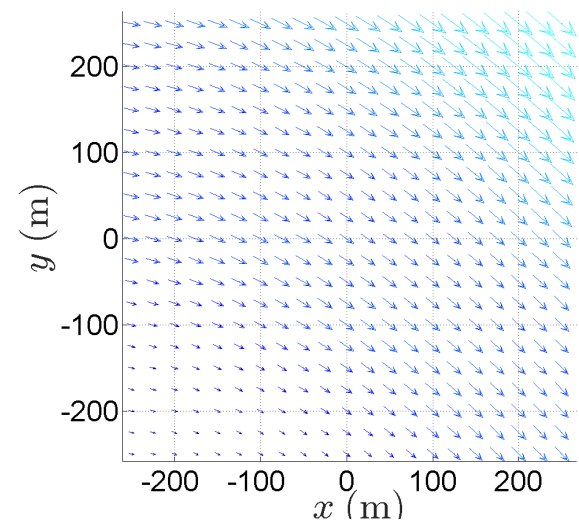

Figure B.20: Current velocity field at $t=9 \mathrm{~h}$ along $z=-250 \mathrm{~m}$.

velocity of the current at its location,

$$
\frac{d \mathbf{p}}{d t}=\left[\begin{array}{lll}
u_{u}(\mathbf{p}, t) & v_{u}(\mathbf{p}, t) & 0
\end{array}\right]^{T}
$$

Floats may be more suited to covering a 3D drifting RoI, as in most places current speed falls off as one descends [42]. Why this outcome tends to arise from physical processes in the ocean was explained in Section 3.4. In Current 2, the current velocity is in the local downstream direction of the jet and decreases in magnitude with increasing depth. As seen in Figure 4.5, nodes drifting on the surface are carried further along eastwards than those remaining closer to the bottom. This suggests that with respect to a point drifting at an intermediate depth, in many currents a float can move ahead of the point by ascending to a depth above it where the current is faster, and lag behind the point, moving in the general opposite direction, by descending 


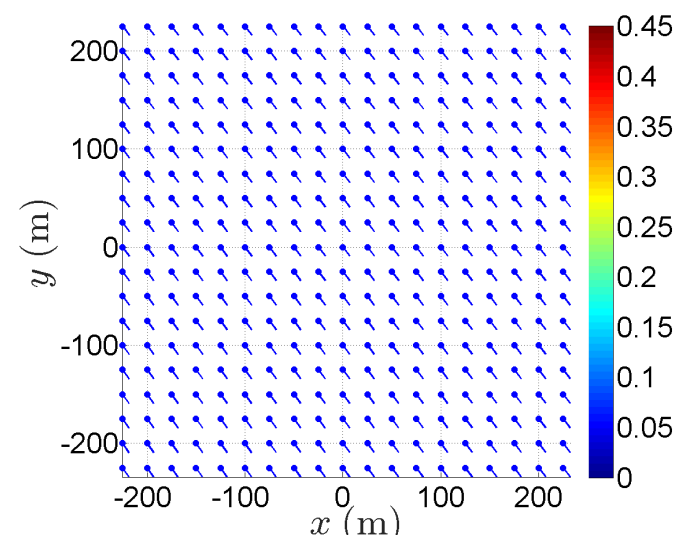

Figure B.22: Controllability map of the currents within the fixed, initially separatrixcentred, box at $t=0 \mathrm{~h}$.

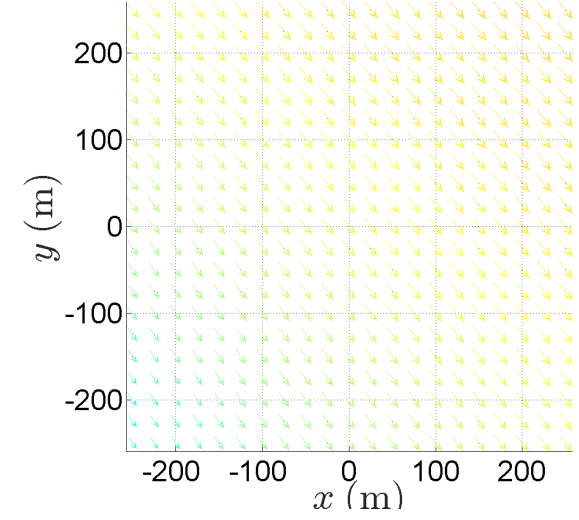

Figure B.24: Current velocity field at $t=0 \mathrm{~h}$ along $z=-250 \mathrm{~m}$.

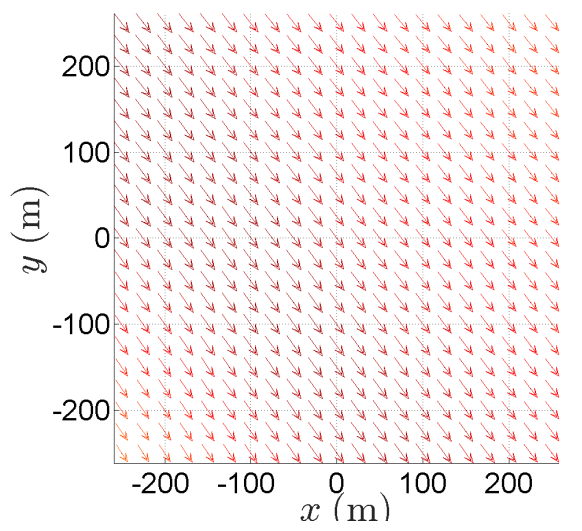

Figure B.23: Current velocity field at $t=0 \mathrm{~h}$ along $z=0 \mathrm{~m}$.

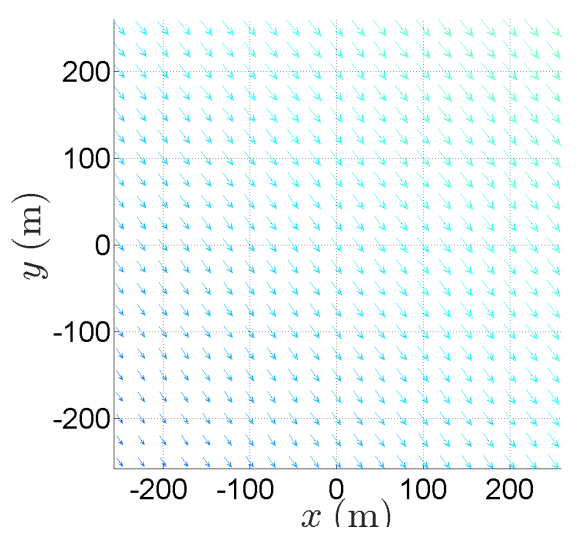

Figure B.25: Current velocity field at $t=0 \mathrm{~h}$ along $z=-500 \mathrm{~m}$.

deeper down where the current is slower. In terms of their displacement from the point as it drifts, floats are 1D controllable if not 2D and 3D controllable, if and it is likely there is any horizontal variation to each side of the motions that are ideally $\pi$ apart and by appeal to infinite profiling velocity per Smith and Dunbabin [181]. Nodes can then spread around and cover a moving RoI centred around and following the point, whereas as seen in the static application floats are limited to moving eastwards and escape the RoI. 


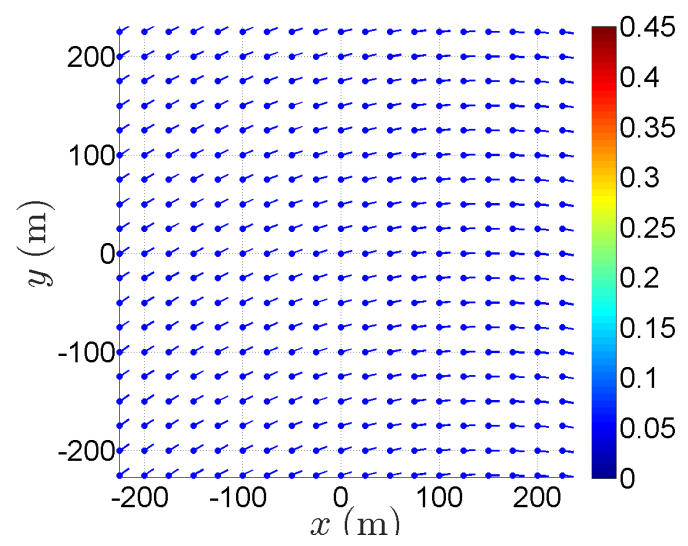

Figure B.26: Controllability map of the currents within the fixed, initially separatrixcentred, box at $t=9 \mathrm{~h}$.

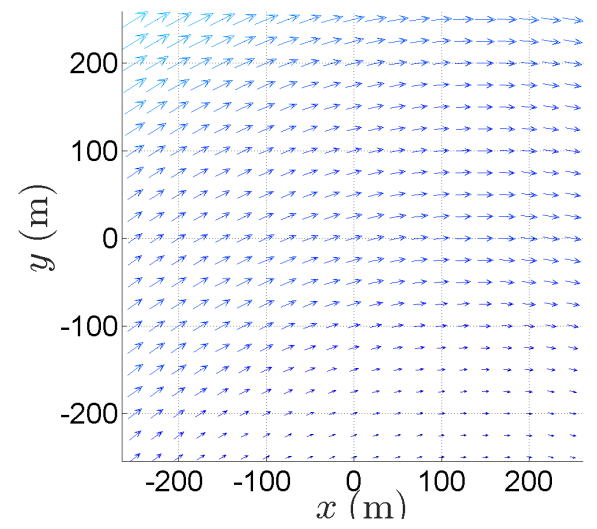

Figure B.28: Current velocity field at $t=9 \mathrm{~h}$ along $z=-250 \mathrm{~m}$.

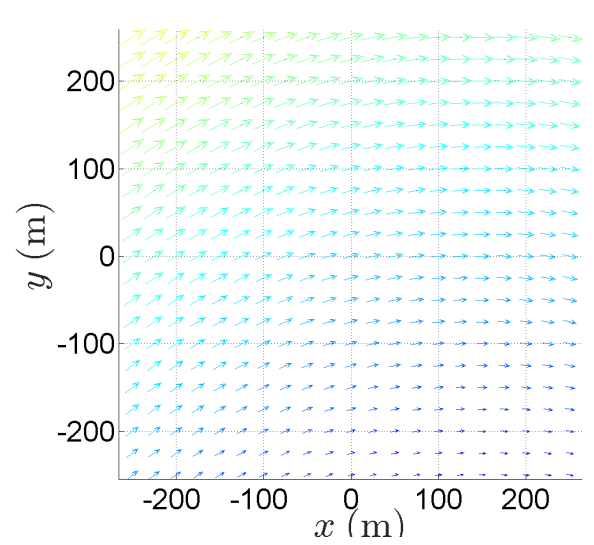

Figure B.27: Current velocity field at $t=9 \mathrm{~h}$ along $z=0 \mathrm{~m}$.

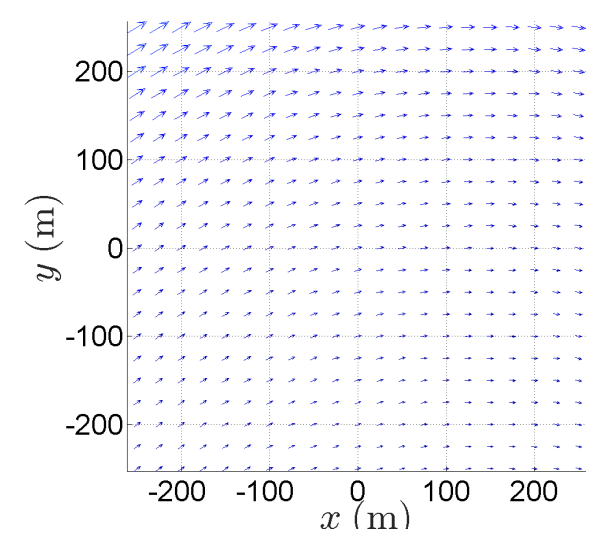

Figure B.29: Current velocity field at $t=9 \mathrm{~h}$ along $z=-500 \mathrm{~m}$.

\section{B.3 Equivalence of controllability problems in cov- ering a drifting and static RoI}

In this section, we show that, because the current velocity is horizontal-only, a choice of co-ordinate system for the body-fixed frame can be found so that the rate of change of a float's body-fixed frame coordinates, its velocity in the body-fixed frame, can be separated out into the sum of the float's unchanged self-propelled profiling velocity in the world frame, $\mathbf{v}_{C}(t)$ and an ambient term $\mathbf{v}_{r U}(t, \mathbf{x})$ independent of the float's mobility choices. We term $\mathbf{v}_{r U}(t, \mathbf{x})$ the relative uncontrollable velocity or relative current velocity. The problem of moving a float from one body-fixed frame coordinate to another body-fixed coordinate is then the same as moving a float between two physical points in the world frame, where $\mathbf{v}_{r U}(t, \mathbf{x})$ acts as the 


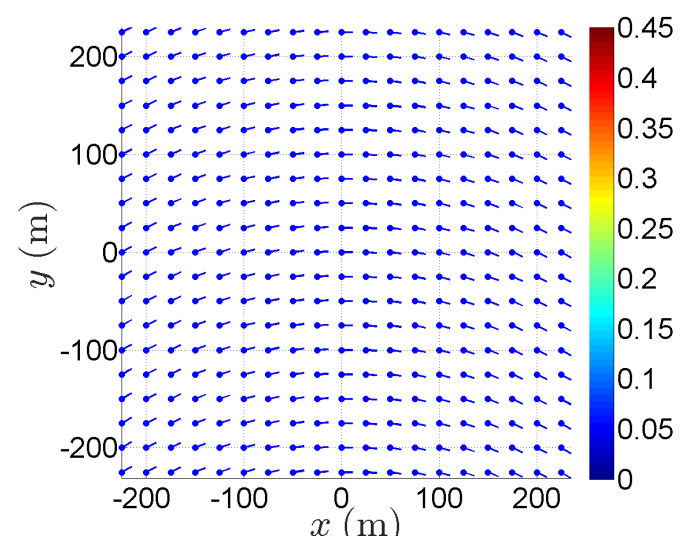

Figure B.30: Controllability map of the currents within the fixed, initially vortex-centred, box at $t=0 \mathrm{~h}$.

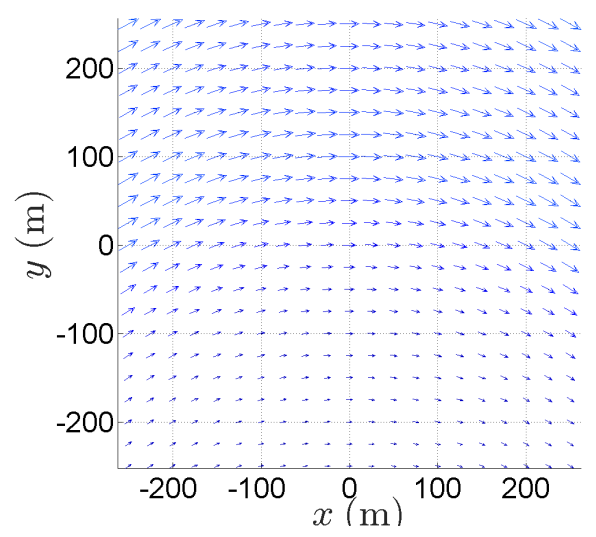

Figure B.32: Current velocity field at $t=0 \mathrm{~h}$ along $z=-250 \mathrm{~m}$.

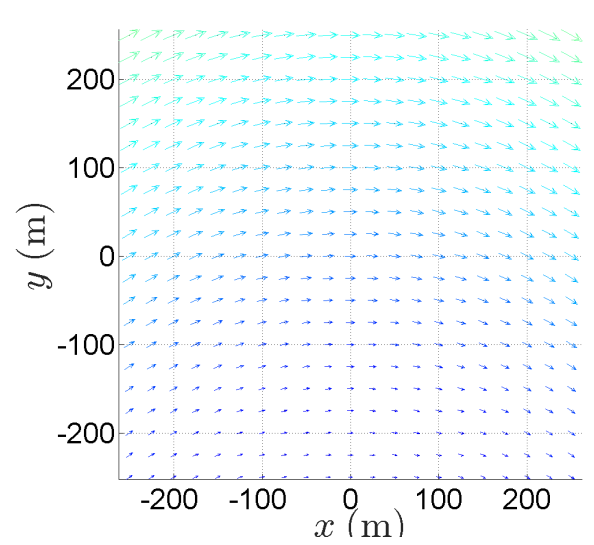

Figure B.31: Current velocity field at $t=0 \mathrm{~h}$ along $z=0 \mathrm{~m}$.

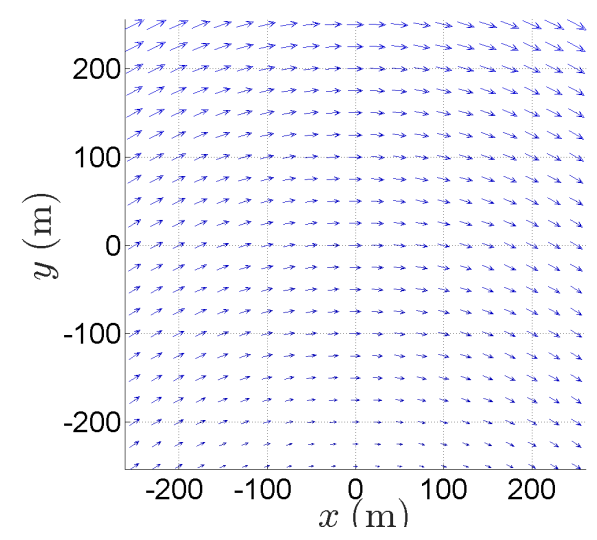

Figure B.33: Current velocity field at $t=0 \mathrm{~h}$ along $z=-500 \mathrm{~m}$.

current velocity field. The float's controllability in the drifting RoI situation can be assessed in an equivalent manner, by evaluating the positive-spanning property of the relative uncontrollable velocity field.

We begin our derivation by defining a basis-defined coordinate system for the body-fixed coordinate frame. The direction of the body-fixed frame's $x$-axis is made to point along the feature's instantaneous direction of motion, the $y$-axis rotated horizontally anticlockwise of the $x$-direction and the $z$-axis points vertically upwards. 


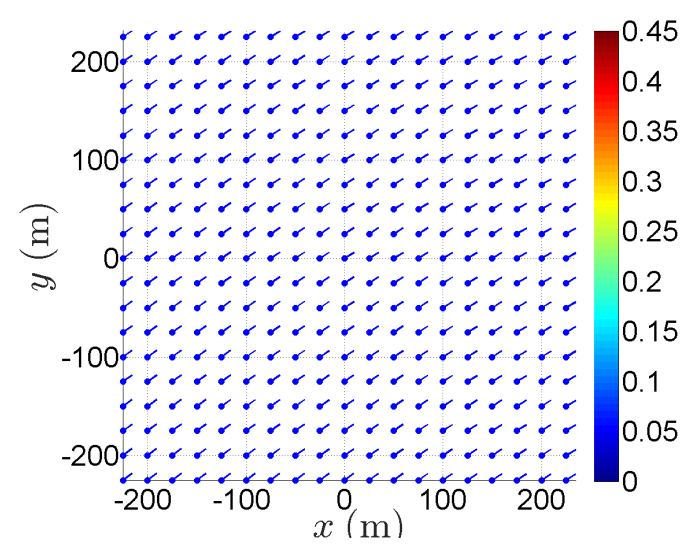

Figure B.34: Controllability map of the currents within the fixed, initially vortex-centred, box at $t=9 \mathrm{~h}$.

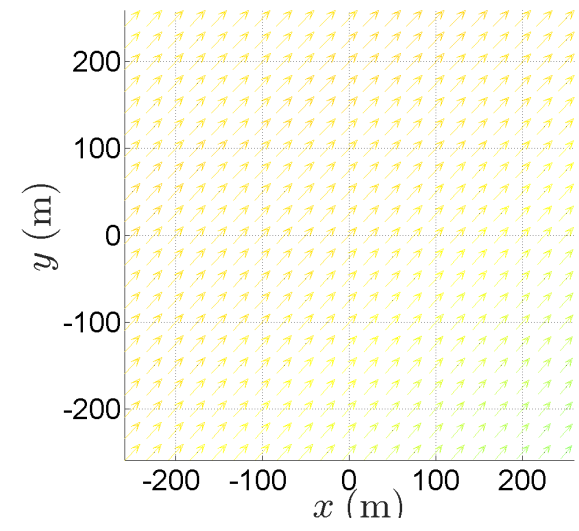

Figure B.36: Current velocity field at $t=9 \mathrm{~h}$ along $z=-250 \mathrm{~m}$.

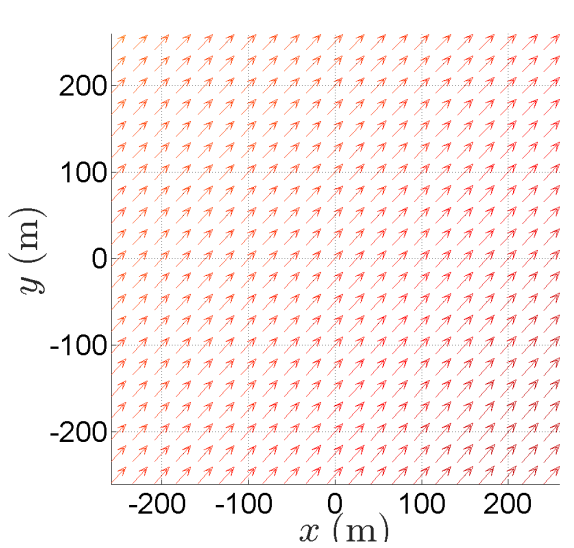

Figure B.35: Current velocity field at $t=9 \mathrm{~h}$ along $z=0 \mathrm{~m}$.

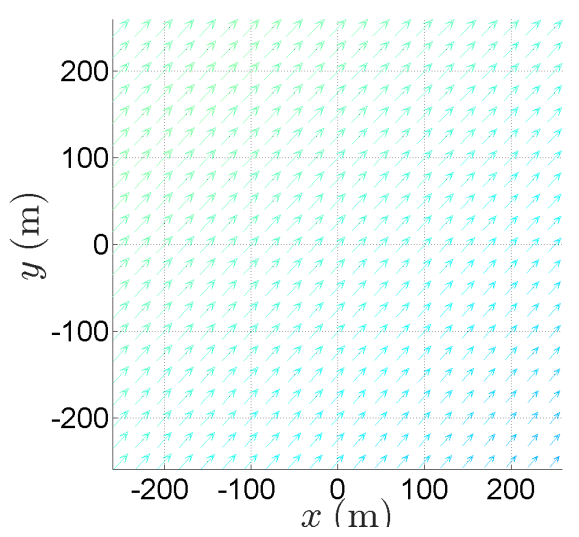

Figure B.37: Current velocity field at $t=9 \mathrm{~h}$ along $z=-500 \mathrm{~m}$.

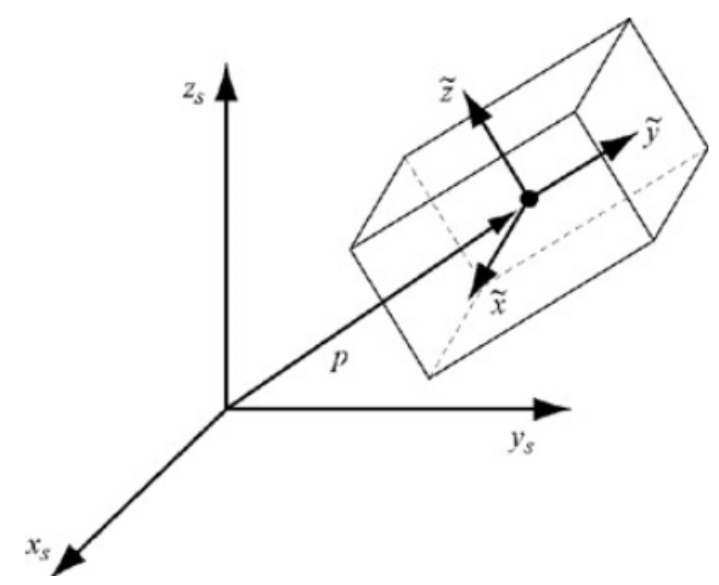

Figure B.38: The body-fixed frame: attached to the rigid body (box) is a body-fixed frame $x-y-z$, with origin p. Shown also is the stationary world frame $x_{s}-y_{s}-z_{s}$. Image from Choset et al [39]. 
This gives the basis vectors as

$$
\begin{aligned}
\stackrel{B}{\mathbf{e}}_{x}(\mathbf{p}, t) & = \begin{cases}{\left[u_{u}(\mathbf{p}, t), v_{u}(\mathbf{p}, t), 0\right]^{T} /\left\|\mathbf{v}_{u}(\mathbf{p}, t)\right\|,} & \left(u_{u}(\mathbf{p}, t), v_{u}(\mathbf{p}, t)\right) \neq(0,0) \\
{[1,0,0]^{T},} & \left(u_{u}(\mathbf{p}, t), v_{u}(\mathbf{p}, t)\right)=(0,0)\end{cases} \\
\stackrel{B}{\mathbf{e}}_{y}(\mathbf{p}, t) & = \begin{cases}{\left[-v_{u}(\mathbf{p}, t), u_{u}(\mathbf{p}, t), 0\right]^{T} /\left\|\mathbf{v}_{u}(\mathbf{p}, t)\right\|,} & \left(u_{u}(\mathbf{p}, t), v_{u}(\mathbf{p}, t)\right) \neq(0,0) \\
{[0,1,0]^{T},} & \left(u_{u}(\mathbf{p}, t), v_{u}(\mathbf{p}, t)\right)=(0,0)\end{cases} \\
\stackrel{\mathrm{e}}{z}_{z} & =[0,0,1]
\end{aligned}
$$

A piecewise function definition is needed in the unlikely case that the current velocity at the drifting feature is exactly zero. The $x$-direction is then arbitrarily set to point east, otherwise it would be undefined.

If the fluid parcel being tracked can be thought of as pointing in the direction of its motion, this co-ordinate system corresponds to conventional axes for a marine craft where velocities in the $x, y$ and $z$ axes are termed surge, sway and heave respectively [116]. Sensed results tagged with the profiler's body-fixed position can then be straightforwardly related to the feature's world position and drift direction at the time.

The stationary frame coordinates of a point, $\stackrel{S}{\mathbf{x}}$, given by standard basis vectors

$$
\begin{aligned}
\stackrel{S}{\mathbf{e}}_{x}(\mathbf{p}, t) & =[1,0,0]^{T} \\
\stackrel{S}{\mathbf{e}}_{y}(\mathbf{p}, t) & =[0,1,0]^{T} \\
\stackrel{\mathrm{e}}{S}_{z} & =[0,0,1]
\end{aligned}
$$

are related to its coordinates in the body-fixed frame, $\stackrel{B}{\mathbf{x}}$, by

$$
\begin{aligned}
\stackrel{S}{\mathbf{X}} & =\mathbf{p}+\left[\begin{array}{lll}
B & B & B \\
\mathbf{e}_{x} & \mathbf{e}_{y} & \mathbf{e}_{z}
\end{array}\right] \stackrel{B}{\mathbf{X}} \\
& =\mathbf{p}+\underset{B}{R} \underset{\mathbf{X}}{S}
\end{aligned}
$$

The matrix

$$
\begin{aligned}
\underset{B}{\stackrel{S}{R}}= & {\left[\begin{array}{lll}
\mathfrak{\mathbf { e }}_{x} & \mathbf{e}_{y} & \mathbf{e}_{z}
\end{array}\right] } \\
& =\left[\begin{array}{lll}
B & B & B \\
e_{x 1} & e_{x 2} & e_{x 3} \\
B & B & B \\
e_{y 1} & e_{y 2} & e_{y 3} \\
B & B & B \\
e_{z 1} & e_{z 2} & e_{z 3}
\end{array}\right]
\end{aligned}
$$

is known as the rotation matrix between frames $B$ and $S$. As the columns of the rotation matrix are the orthonormal basis vectors, $\underset{B}{R}$ is orthogonal thus $\left(\begin{array}{c}S \\ R \\ B\end{array}\right)^{-1}=$ 
$\left(\begin{array}{l}S \\ R \\ B\end{array}\right)^{T}[129]$. Hence,

$$
\stackrel{B}{\mathbf{X}}=\left(\begin{array}{c}
S \\
R \\
B
\end{array}\right)^{T}(\stackrel{S}{\mathbf{X}}-\mathbf{p})
$$

We now derive the float's velocity in the body-fixed frame, that is the rate of change of its body-fixed position coordinates. This depends on the rate of change of the body-fixed frame's orientation. The float's velocity in the body-fixed frame can be found by differentiating the position equation (B.5) with respect to time, which gives [91]

$$
\begin{aligned}
& \stackrel{S}{\mathbf{v}}=\frac{d \stackrel{S}{\mathbf{x}}}{d t} \\
& =\frac{d \mathbf{p}}{d t}+\frac{d}{d t}[\stackrel{S}{R} \underset{B}{B} \mathbf{x}] \\
& =\frac{d \mathbf{p}}{d t}+\frac{d}{d t}[\stackrel{S}{R}] \underset{B}{B} \mathbf{x}+\stackrel{S}{R} \frac{d \mathbf{x}}{d t} \\
& =\frac{d \mathbf{p}}{d t}+\frac{d}{d t}[\stackrel{S}{R}]\left(\stackrel{S}{R}{ }_{B}^{R}\right)^{T}(\stackrel{S}{\mathbf{x}}-\mathbf{p})+\underset{B}{R} B \\
& =\frac{d \mathbf{p}}{d t}+\tilde{\omega}(\stackrel{S}{\mathbf{X}}-\mathbf{p})+\stackrel{S}{R} \underset{B}{B} \\
& =\frac{d \mathbf{p}}{d t}+\omega \times(\stackrel{S}{\mathbf{X}}-\mathbf{p})+\stackrel{S}{R} \underset{B}{B}
\end{aligned}
$$

The quantity

$$
\begin{aligned}
\tilde{\omega} & =\frac{d}{d t}[\stackrel{S}{R}]\left(\begin{array}{l}
S \\
R \\
B
\end{array}\right)^{T} \\
& =\left[\begin{array}{ccc}
0 & -\omega_{3} & \omega_{2} \\
\omega_{3} & 0 & -\omega_{1} \\
-\omega_{2} & \omega_{1} & 0
\end{array}\right]
\end{aligned}
$$

is termed the angular velocity matrix, where

$$
\omega=\left[\begin{array}{lll}
\omega_{1} & \omega_{2} & \omega_{3}
\end{array}\right]^{T}
$$

is known as the angular velocity vector of the frame $B$ with respect to $S$ [91]. 
The float's velocity in the body-fixed frame is therefore

$$
\begin{aligned}
& \stackrel{B}{\mathbf{v}}=\left(\begin{array}{c}
S \\
R \\
B
\end{array}\right)^{T}\left(\stackrel{S}{\mathbf{V}}-\frac{d \mathbf{p}}{d t}-\omega \times(\stackrel{S}{\mathbf{X}}-\mathbf{p})\right)
\end{aligned}
$$

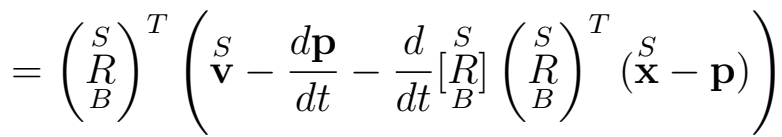

$$
\begin{aligned}
& =\left(\begin{array}{c}
S \\
R \\
B
\end{array}\right)^{T}\left(\stackrel{S}{\mathbf{v}}-\frac{d \mathbf{p}}{d t}-\frac{d}{d t}[\stackrel{S}{R}]_{B}^{B} \mathbf{x}\right)
\end{aligned}
$$

The node's inertial velocity is

$$
\stackrel{S}{\mathbf{v}}(t)=\mathbf{v}_{\mathrm{C}}(t)+\mathbf{v}_{\mathrm{U}}(t \stackrel{S}{\mathbf{x}})
$$

Substituting this into (B.11) gives

$$
\begin{aligned}
& \stackrel{B}{\mathbf{v}}(t)=\left(\begin{array}{c}
S \\
R \\
B
\end{array}\right)^{T}\left(\stackrel{S}{\mathbf{v}}(t)-\frac{d \mathbf{p}}{d t}-\frac{d}{d t}[\stackrel{S}{R}] \underset{B}{\mathbb{X}}\right) \\
& =\left(\begin{array}{l}
\stackrel{S}{R} \\
B
\end{array}\right)^{T}\left(\mathbf{v}_{\mathrm{C}}(t)+\mathbf{v}_{\mathrm{U}}(t, \stackrel{S}{\mathbf{x}})-\frac{d \mathbf{p}}{d t}-\left(\frac{d}{d t}[\stackrel{S}{R}]\right) \stackrel{B}{\mathbf{x}}\right) \\
& =\left(\begin{array}{l}
S \\
R \\
B
\end{array}\right)^{T} \mathbf{v}_{C}(t)+\left(\begin{array}{l}
S \\
R \\
B
\end{array}\right)^{T}\left(\mathbf{v}_{U}(t, \stackrel{S}{\mathbf{x}})-\frac{d \mathbf{p}}{d t}-\left(\frac{d}{d t}[\stackrel{S}{R}]{ }_{B}\right) \stackrel{B}{\mathbf{x}}\right) \\
& =\mathbf{v}_{r C}(t)+\mathbf{v}_{r U}(t, \stackrel{B}{\mathbf{x}})
\end{aligned}
$$

The node's body-fixed frame velocity can be thought of as the sum of a relative controllable velocity

$$
\mathbf{v}_{r C}(t)=(\stackrel{S}{R}(t))^{T} \mathbf{v}_{C}(t)
$$

and a relative uncontrollable (current) velocity, given by a relative current velocity field on the space of body-fixed frame position vectors ,

$$
\begin{aligned}
& \mathbf{v}_{r U}(t, \stackrel{B}{\mathbf{x}})=(\stackrel{\stackrel{S}{R}}{\underset{B}{R}})^{T}\left(\mathbf{v}_{U}(t, \stackrel{S}{\mathbf{X}})-\frac{d \mathbf{p}}{d t}-\left(\frac{d}{d t}[\stackrel{S}{R}]\right) \underset{B}{\mathbf{X}}\right) \\
& =\left(\begin{array}{l}
\stackrel{S}{R} \\
B
\end{array}\right)^{T}\left(\mathbf{v}_{u}(t, \stackrel{S}{R} \underset{B}{B}+\mathbf{p})-\frac{d \mathbf{p}}{d t}-\left(\frac{d}{d t}[\stackrel{S}{R}]\right) \underset{\mathbf{X}}{\mathbf{X}}\right)
\end{aligned}
$$

For two nodes at the same position (inertial or body-fixed frame) at the same time, both experience the same relative uncontrollable velocity but their relative controllable velocity may vary depending on the different actuation control between them. 
For the co-ordinate system in (B.3), the rotation matrix is given by

$$
\underset{B}{S}=\left[\begin{array}{lll}
B & B & B \\
e_{x 1} & e_{x 2} & e_{x 3} \\
B & B & B \\
e_{y 1} & e_{y 2} & e_{y 3} \\
B & B & B \\
e_{z 1} & e_{z 2} & e_{z 3}
\end{array}\right]=\left[\begin{array}{lll}
\frac{u_{u}(\mathbf{p}, t)}{\left\|\mathbf{v}_{u}(\mathbf{p}, t)\right\|} & \frac{v_{u}(\mathbf{p}, t)}{\left\|\mathbf{v}_{u}(\mathbf{p}, t)\right\|} & 0 \\
\frac{-v_{u}(\mathbf{p}, t)}{\left\|\mathbf{v}_{u}(\mathbf{p}, t)\right\|} & \frac{u_{u}(\mathbf{p}, t)}{\left\|\mathbf{v}_{u}(\mathbf{p}, t)\right\|} & 0 \\
0 & 0 & 0
\end{array}\right]
$$

and

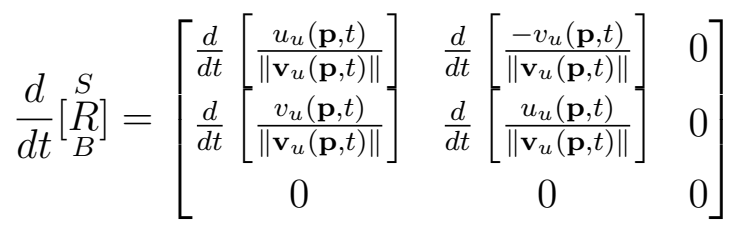

The relative controllable velocity of the float is then simply its controllable velocity in the inertial frame, that is its vertical actuated velocity, since

$$
\mathbf{v}_{C}(t)=\left[\begin{array}{lll}
0 & 0 & w_{C}(t)
\end{array}\right]^{T}
$$

and so

$$
\begin{aligned}
\mathbf{v}_{r C}(t)= & (\stackrel{S}{R}(t))^{T} \mathbf{v}_{C}(t) \\
& =\left[\begin{array}{l}
\frac{u_{u}(\mathbf{p}, t)}{\left\|\mathbf{v}_{u}(\mathbf{p}, t)\right\|} \times 0+\frac{-v_{u}(\mathbf{p}, t)}{\left\|\mathbf{v}_{u}(\mathbf{p}, t)\right\|} \times 0+0 \times w_{C}(t) \\
\frac{v_{u}(\mathbf{p}, t)}{\left\|\mathbf{v}_{u}(\mathbf{p}, t)\right\|} \times 0+\frac{u_{u}(\mathbf{p}, t)}{\left\|\mathbf{v}_{u}(\mathbf{p}, t)\right\|} \times 0+0 \times w_{C}(t) \\
0 \times 0+0 \times 0+1 \times w_{C}(t)
\end{array}\right] \\
& =\left[\begin{array}{lll}
0 & 0 & w_{C}(t)
\end{array}\right]^{T} \\
& =\mathbf{v}_{C}(t)
\end{aligned}
$$

The rate of change of a float's body-fixed coordinates is thus

$$
\stackrel{B}{\mathbf{v}}(t)=\mathbf{v}_{C}(t)+\mathbf{v}_{r U}(t, \stackrel{B}{\mathbf{x}})
$$

where

$$
\mathbf{v}_{r U}(t, \stackrel{B}{\mathbf{X}})=\left(\begin{array}{l}
\stackrel{S}{R} \\
B
\end{array}\right)^{T}\left(\mathbf{v}_{u}(t, \underset{B}{\stackrel{S}{R} \mathbf{X}}+\mathbf{p})-\frac{d \mathbf{p}}{d t}-\left(\frac{d}{d t}[\stackrel{S}{R}]\right) \underset{\mathbf{X}}{\mathbf{X}}\right)
$$

is the relative current velocity, and

$$
\mathbf{v}_{C}(t)=\left[\begin{array}{lll}
0 & 0 & w_{C}(t)
\end{array}\right]^{T}
$$

is the profiling velocity of the float in both the static and drifting frames. Note that this only applies for flows where the current velocity is horizontal-only like Current 2 and for the choice of basis-defined body-fixed frame coordinate system in (B.3). 


\section{B.4 Controllability analysis of Current 2 in the drifting frame of reference}

To assess the ability of profiling floats to cover a drifting RoI in Current 2, controllability maps of the relative current velocity fields were generated after Section B.1. Drifting RoIs were selected in the jet, separatrix and vortex at $t=0 \mathrm{~h}$, so all distinctive regimes of the current are included. The RoIs consist of a $500 \mathrm{~m} \times 500 \mathrm{~m} \times 500 \mathrm{~m}$ box centred and drifting with a feature at mid-depth, $250 \mathrm{~m}$. Figure B.39 shows the evolution of the RoI's positions over $12 \mathrm{~h}$. Drawn through each box is the $x$-axis in the body-fixed frame and the half-line is the positive $y$-axis, to show the orientation of the frame and the velocity of the tagged feature at the time.

Figures B.40, B.48, and B.56 display controllability maps of the relative current velocity field at $t=0 \mathrm{~h}$ for each of the RoIs. At all positions a current along the profile has a negative $x$ - and another current has a positive $x$-component. In the vessel-based body-fixed frame, this corresponds to depths that will result in a float lagging behind and moving ahead of the feature, validating the thought process in Section B.2. However, nowhere does the current positively span the 2D plane, when this was considered likely. It seems that the spread of velocities at each position is restricted to one side of the $y$-axis. This is clearly visible in Figure B.56, where the currents form a "fantail" pattern. It is conjectured that this is due to the sense of curvature of the meandering jet current not changing across the small RoI.

Note that Current 2 is not a completely accurate description of coastal currents, being based on the averaged geostrophic current. Small-scale current components typical of coastal environments such as tidal and inertial currents [177], as discussed in Section 3.7, are not represented in the model. Had these small-scale components been included, for instance by adding a stochastic random walk component on top of the field after Chiswell and Rickard [37], these would likely have resulted in adjacent currents at each point being less than $\pi$ apart and thus positively spanning the plane. The existing current vectors are already directed generally opposite to each other. The additional variation of the random walk component would tend to push the velocity distribution over the $\pi$ threshold. Before discounting using floats to cover moving RoIs, the controllability analysis done here should be repeated with in-situ current measurements to confirm or deny these results.

Scheme 1 was proposed with the requirement that the current exhibited controllability and non-time-varying, non-horizontally spatially varying properties, none 

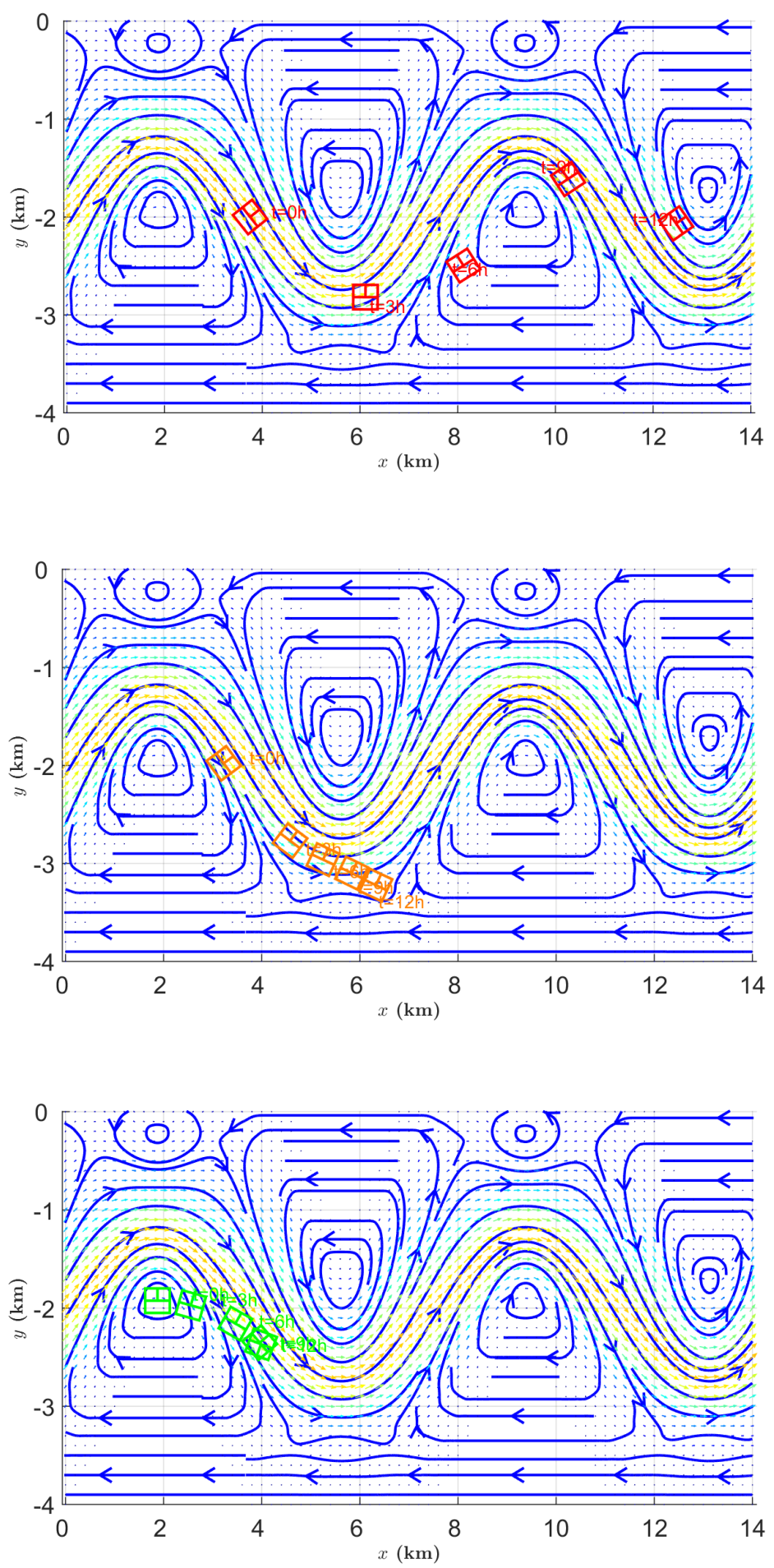

Figure B.39: Drifting RoI tagging an initially (from top to bottom) a) jet-centred, b) separatrixcentred and c) vortex-centred drifting feature at $t=0,3,6,9,12 \mathrm{~h}$. Shown alongside is the current velocity in the world frame, and comoving frame streamlines at $t=0 \mathrm{~h}, 250 \mathrm{~m}$ depth. 


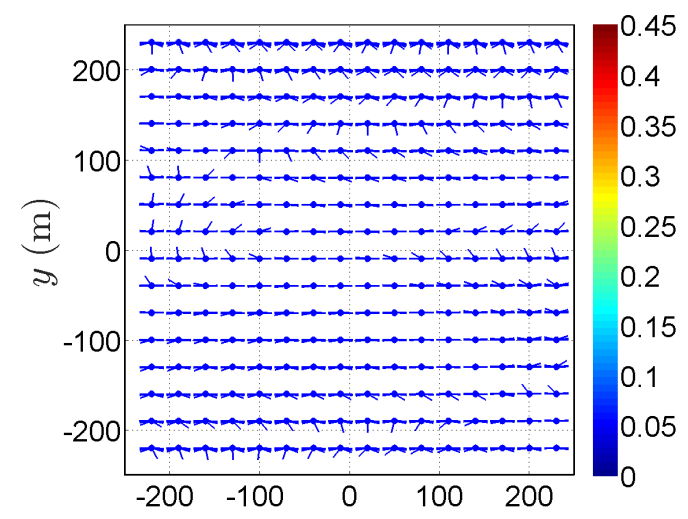

Figure B.40: Drifting RoI controllability map of the currents within the initially jet-centred, box at $t=0 \mathrm{~h}$.

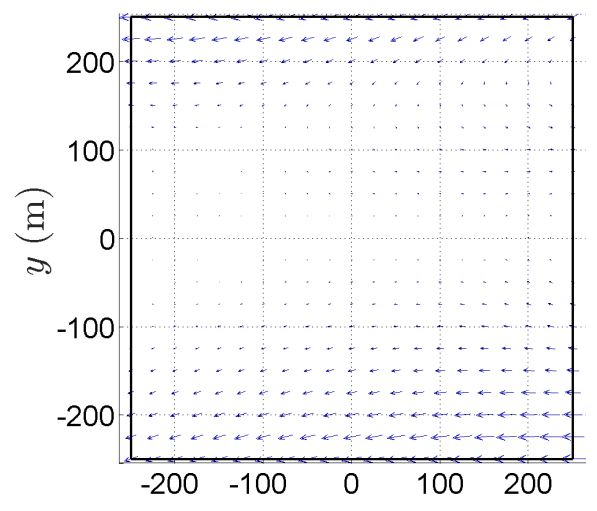

Figure B.42: Current velocity field at $t=0 \mathrm{~h}$ along $z=-250 \mathrm{~m}$. Surge and sway axes.

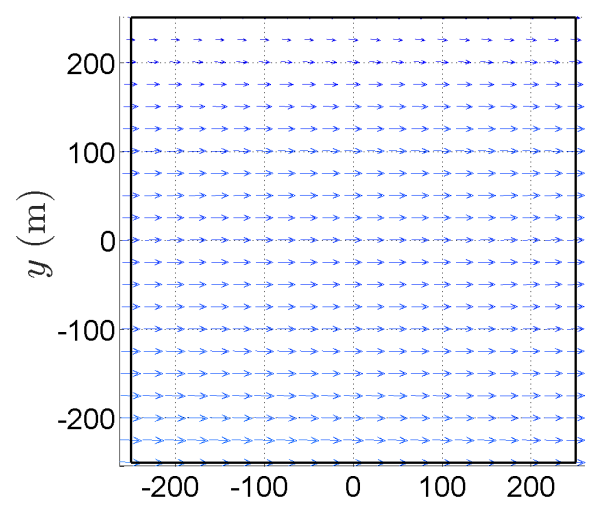

Figure B.41: Current velocity field at $t=0 \mathrm{~h}$ along $z=0 \mathrm{~m}$.

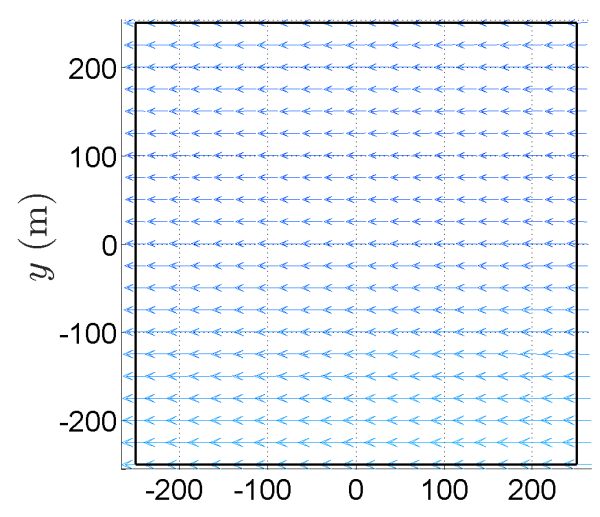

Figure B.43: Current velocity field at $t=0 \mathrm{~h}$ along $z=-500 \mathrm{~m}$. Surge and sway axes.

of which occur in this case. The velocity field is shown to vary across the RoI in Figures B.41-B.63, where the velocities at each layer are non-uniform. The visible differences between the layer velocity fields at times $t=0 \mathrm{~h}$ and $t=12 \mathrm{~h}$ also indicate that the current is time-varying.

The lack of controllability in the maps is first considered in isolation, that is to say the current profile at each float's location is assumed to hold throughout the region. In Scheme 1, area coverage is obtained as a potential by-product of floats being able to periodically return to target positions. When a node passed through a goal location, reliance was placed on other targets, which also have profilers assigned to them and thus nodes regularly passing through them, having a profiler close by. These targets were distributed across the network. Hence, the individual float movements would tend to frequently result in a set of nodes spread throughout the 


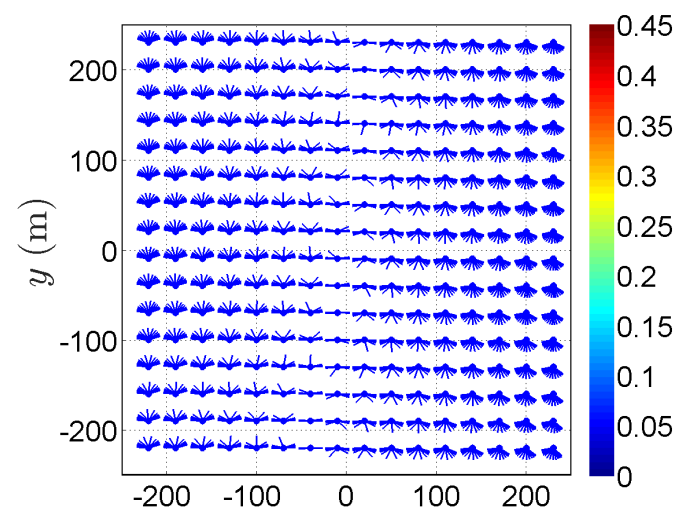

Figure B.44: Drifting RoI controllability map of the currents within the initially jet-centred, box at $t=9 \mathrm{~h}$.

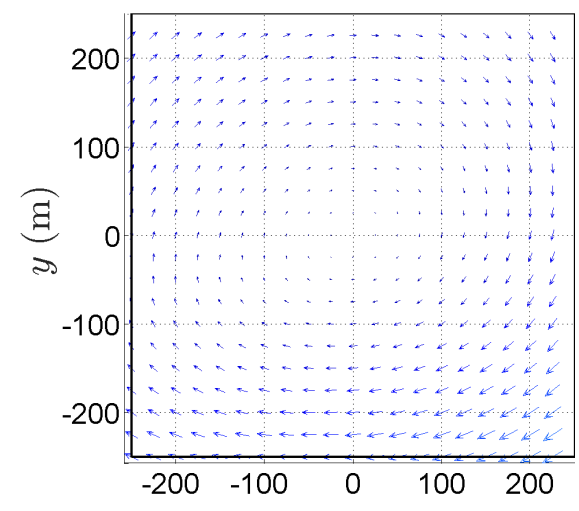

Figure B.46: Current velocity field at $t=9 \mathrm{~h}$ along $z=-250 \mathrm{~m}$.

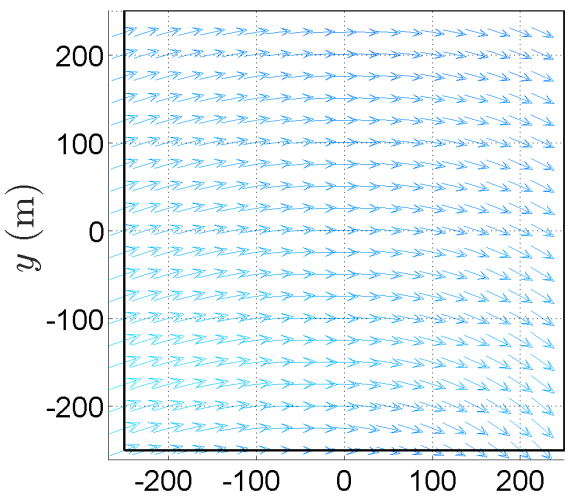

Figure B.45: Current velocity field at $t=9 \mathrm{~h}$ along $z=0 \mathrm{~m}$.

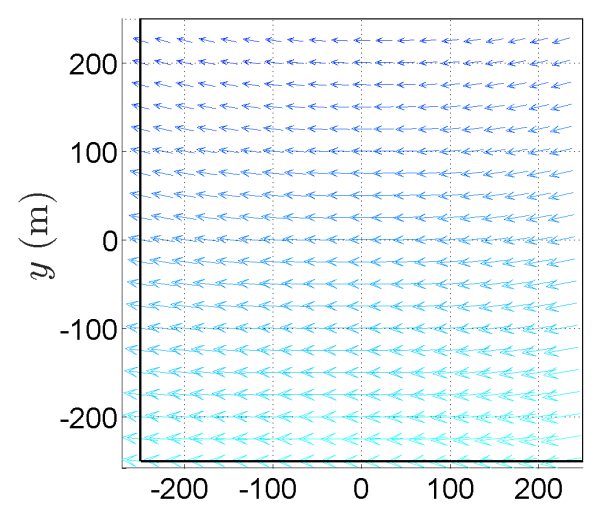

Figure B.47: Current velocity field at $t=9 \mathrm{~h}$ along $z=-500 \mathrm{~m}$.

network and covering a sizeable portion of the environment.

This particular current, which does not positively span the plane, means that the convex optimization problem cannot be solved to find a trajectory that allows the float to pass exactly through its target. However, the solver will still find the trajectory that minimizes the displacement of the float from the goal. Floats are initially assigned targets based on a minimax algorithm, so that their $x$ - and $y$ displacements from their goals should be small. With the currents along the profile spanning the $x$-axis, a feasible trajectory exists from each node that completely eliminates the error in the $x$-direction with an accumulating error in the $y$-direction, the $y$-components of the vectors being all of one sign. The displacement of this trajectory from the target sets an upper bound on that found by the solver. Given the observed currents that have only small $y$-components, such as in Figure B.40, the 


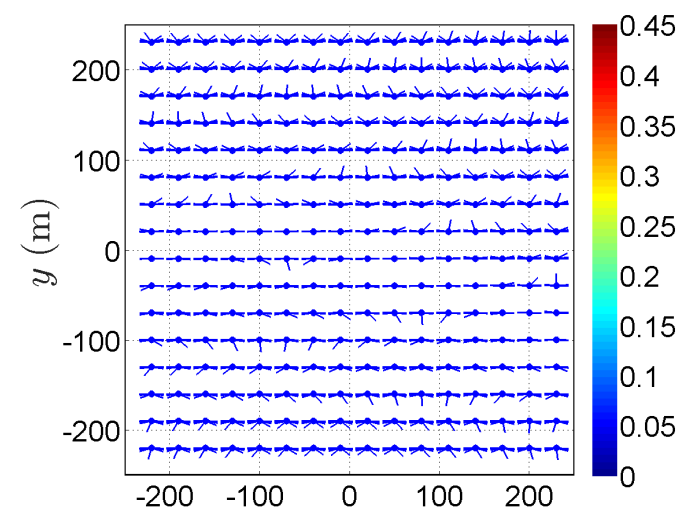

Figure B.48: Drifting RoI controllability map of the currents within the initially separatrixcentred, box at $t=0 \mathrm{~h}$.

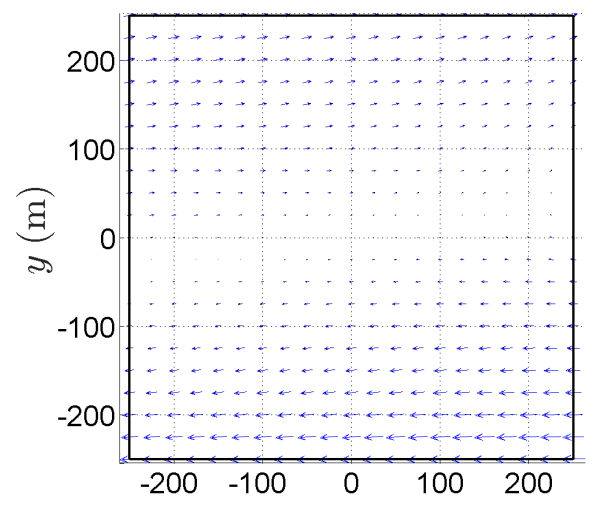

Figure B.50: Current velocity field at $t=0 \mathrm{~h}$ along $z=-250 \mathrm{~m}$. Surge and sway axes.

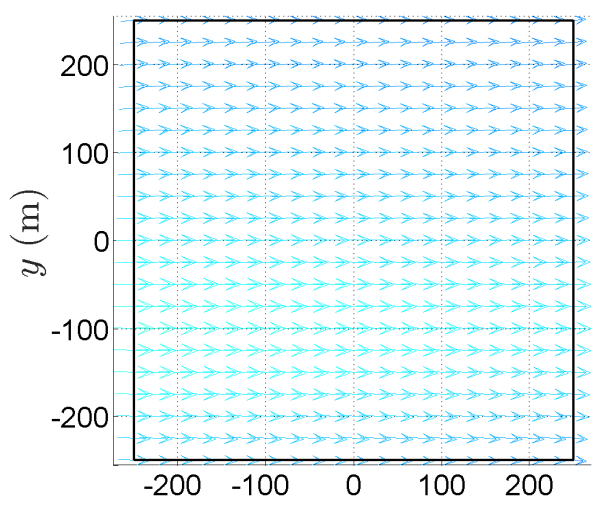

Figure B.49: Current velocity field at $t=0 \mathrm{~h}$ along $z=0 \mathrm{~m}$.

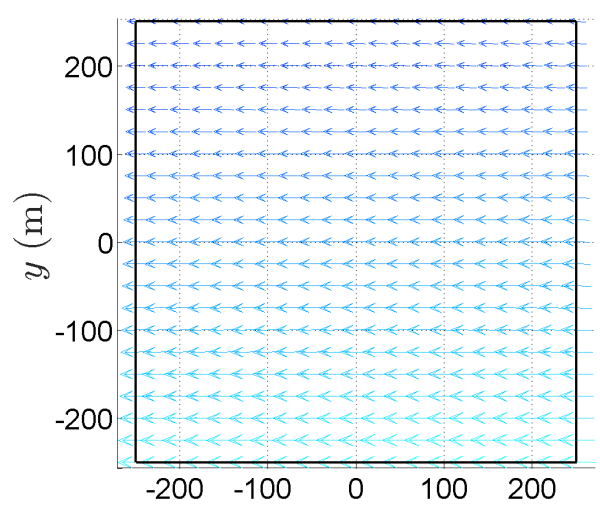

Figure B.51: Current velocity field at $t=0 \mathrm{~h}$ along $z=-500 \mathrm{~m}$. Surge and sway axes.

closest distance the float can come within its target need only increase slightly per cycle. While this scheme may not be able to prevent the node eventually moving out of sensing range of its target on its closest approach, it can, even in this non-ideal current, prolong the time a float frequents positions near to its target. Compared to not profiling, where after an initial period inside the RoI a float higher up or lower down steadily moves ahead or is left behind, nodes are at least able to return to the RoI and provide coverage at later times. Also, by guiding nodes close to points throughout the RoI, at any one time profilers are likely to be spread out across the region in the process of arriving at or leaving their targets and covering a wide area.

This only occurs if the trajectories are accurate. With the currents being timevarying and spatially-varying, the displacements anticipated of the trajectory by the convex optimization problem formulation will be in error. The actual paths taken by 


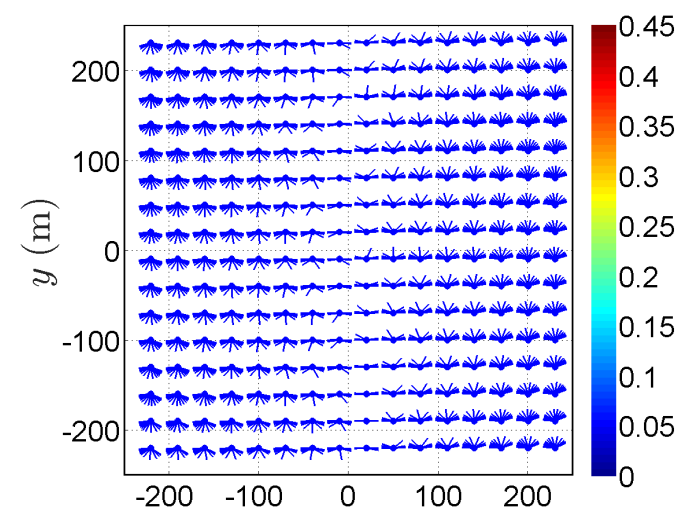

Figure B.52: Drifting RoI controllability map of the currents within the initially separatrixcentred, box at $t=9 \mathrm{~h}$.

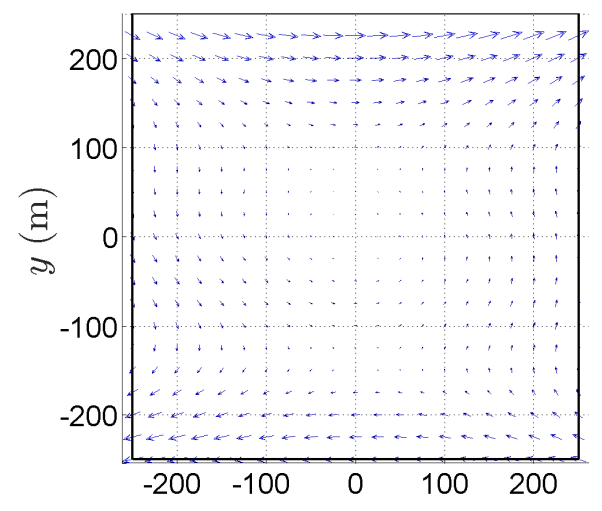

Figure B.54: Current velocity field at $t=9 \mathrm{~h}$ along $z=-250 \mathrm{~m}$.

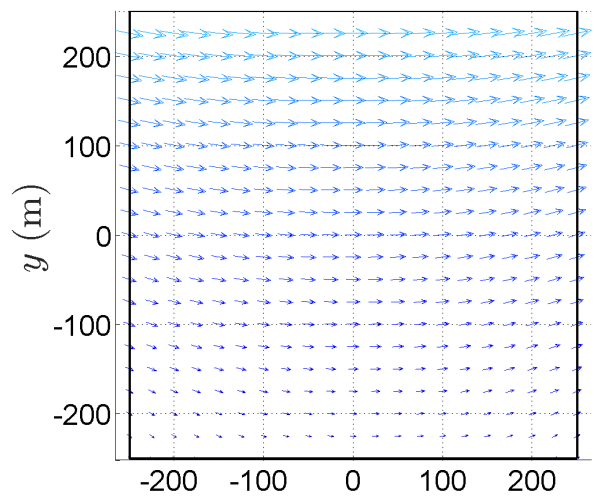

Figure B.53: Current velocity field at $t=9 \mathrm{~h}$ along $z=0 \mathrm{~m}$.

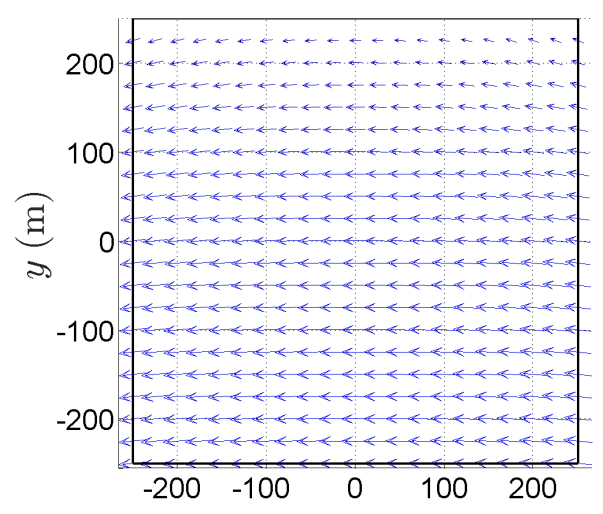

Figure B.55: Current velocity field at $t=9 \mathrm{~h}$ along $z=-500 \mathrm{~m}$.

the nodes may leave portions of the network uncovered and may remain outside of the RoI entirely. 


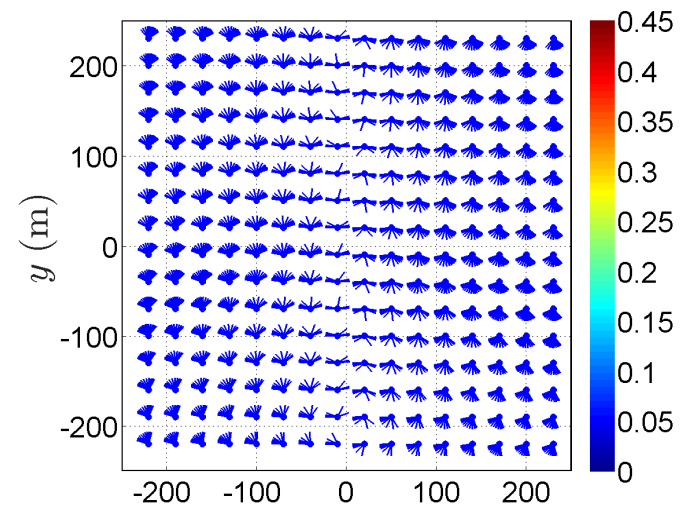

Figure B.56: Drifting RoI controllability map of the currents within the initially vortex-centred, box at $t=0 \mathrm{~h}$.

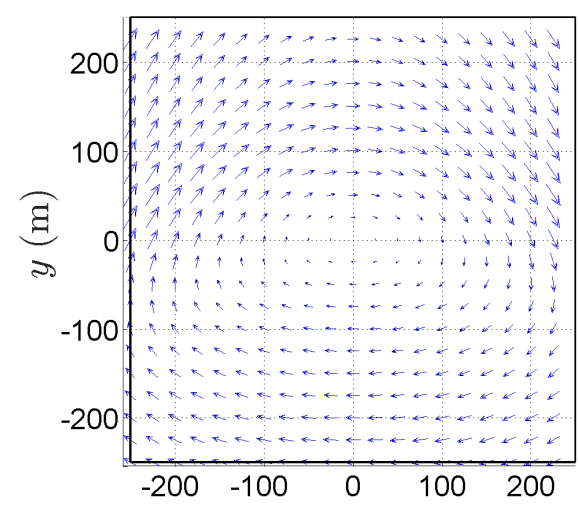

Figure B.58: Current velocity field at $t=0 \mathrm{~h}$ along $z=-250 \mathrm{~m}$. Surge and sway axes.

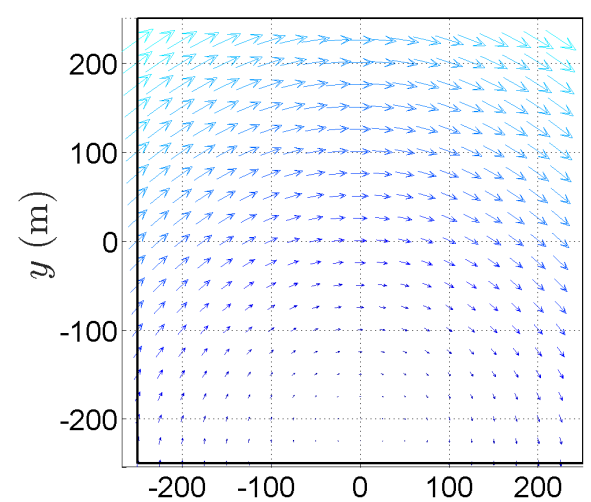

Figure B.57: Current velocity field at $t=0 \mathrm{~h}$ along $z=0 \mathrm{~m}$.

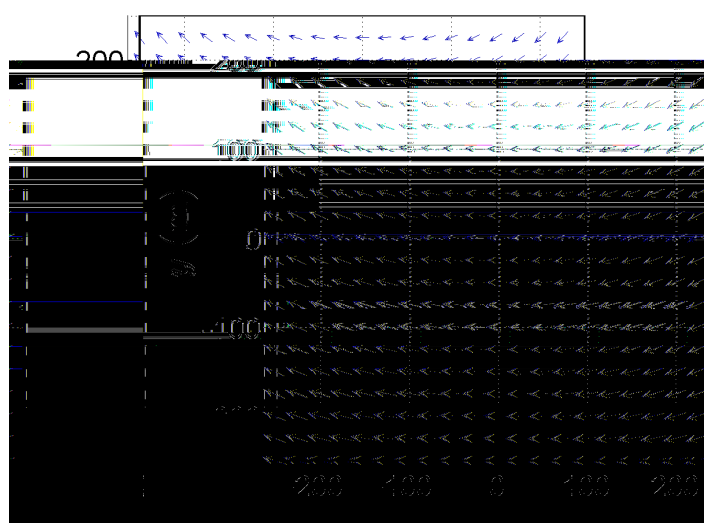

Figure B.59: Current velocity field at $t=0 \mathrm{~h}$ along $z=-500 \mathrm{~m}$. Surge and sway axes. 


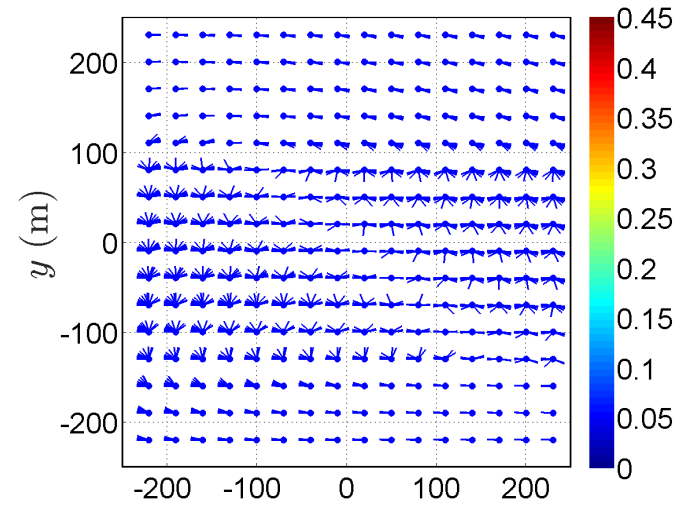

Figure B.60: Drifting RoI controllability map of the currents within the initially vortex-centred, box at $t=9 \mathrm{~h}$.

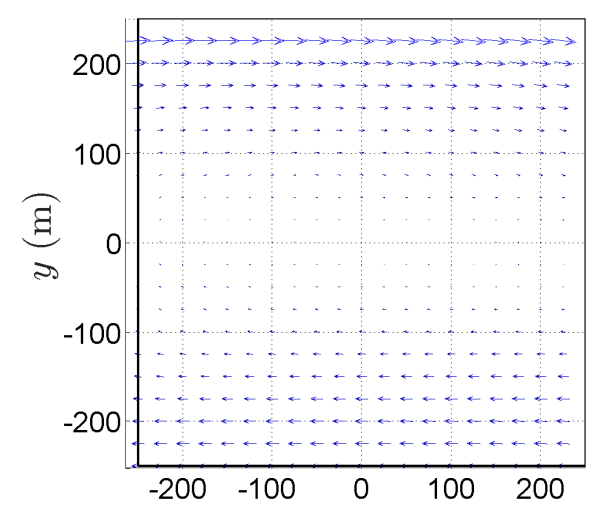

Figure B.62: Current velocity field at $t=9 \mathrm{~h}$ along $z=-250 \mathrm{~m}$.

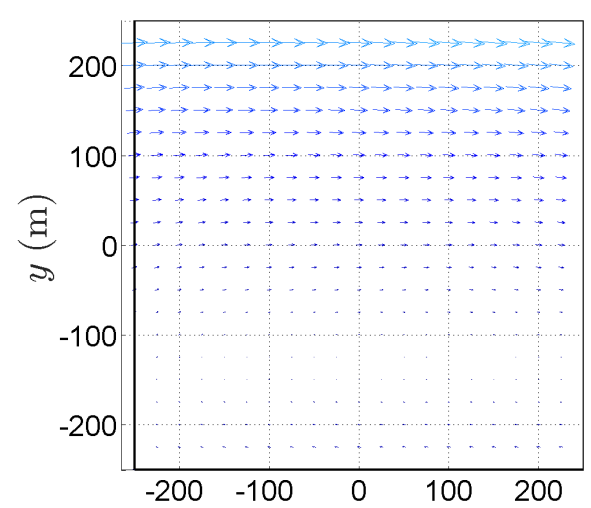

Figure B.61: Current velocity field at $t=9 \mathrm{~h}$ along $z=0 \mathrm{~m}$.

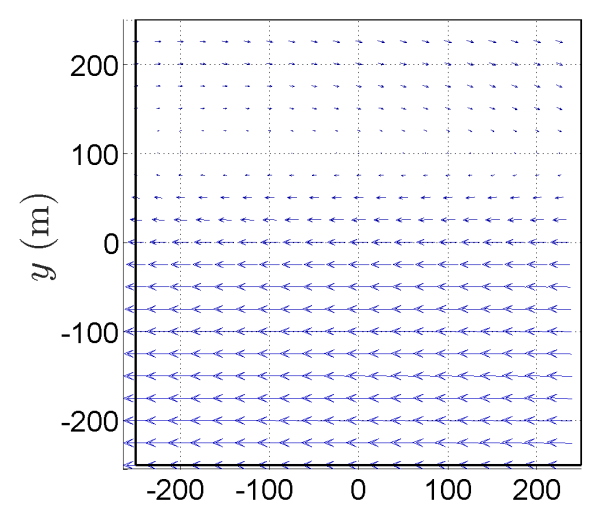

Figure B.63: Current velocity field at $t=9 \mathrm{~h}$ along $z=-500 \mathrm{~m}$. 


\section{Appendix C}

\section{Network mobility simulator design}

This appendix details the code architecture employed in our MATLAB-based discreteevent network mobility simulator. We coded this simulator to carry out our float node movement scheme performance study in Chapter 6 .

This appendix is structured as follows. Earlier routing protocols simulations in Chapter 4 were conducted in QualNet, a network protocol simulation software platform implemented in $\mathrm{C} / \mathrm{C}++$. The motivation for switching to a custom network mobility simulator coded in MATLAB is explained in Section C.1. The simulator we developed is a discrete-event simulator. In this type of simulator, the simulator maintains a queue of events that are scheduled to execute at particular simulation times [159]. Events are dynamically added to the queue during the course of the simulation. The events are executed in sequential time order by the simulator. During the execution of an event, the network state is updated based on the nature of the scheduled event, and upon completion the simulator moves to the next event in time. In this way, the simulator advances discretely rather than continuously in time from one event to the next. Section C.2 details how events are implemented in our code. After this, Section C.3 covers how the node movement scheme that we proposed, Scheme 1, is implemented in the simulator via discrete events. The simulation record output by the simulator is described in Section C.4.

\section{C.1 Motivation}

We develop our custom network mobility simulator in MATLAB rather than modifying QualNet, as QualNet does not natively support dynamic mobility. Node mobility in QualNet is supported by being able to specify waypoint files that are loaded at the start of the simulation and define the motion of each node for the 
entire simulation duration [166]. We were able to carry out our routing protocol simulations in QualNet, as the nodes were only drifting with the currents and had no actuated velocity of their own. The displacement due to the currents could be pre-generated and written into waypoint files. However, our node movement scheme executes different node mobility in response to in-environment events. This dynamic mobility cannot be specified in waypoint files.

It was envisioned that as the mobility functionality would need to be developed from scratch in either case, a simulator coded in MATLAB would be used since it would take around the same time to build a simulator in MATLAB than modify QualNet. MATLAB was already being utilized to process and graph results, and with this change all coding could be done more conveniently in one environment and without having to deal with the complications of converting between $\mathrm{C}++$ and MATLAB data types. Furthermore, the simulator could draw upon MATLAB's extensive in-built and Matlab Central File Exchange community libraries, initially for its numerical integration functions [126] and later to implement a convex optimization problem solver [8], simulated annealing and concepts from computational geometry [2].

\section{C.2 Event simulation}

The implementation of events in our simulator is now given. The simulator consists of four main components:

- A single NetworkMobilitySimulator object keeping track of the simulation time and containing a description of the current velocity field and a priority queue of NetworkEvent objects. Each NetworkEvent object includes a time, the name of a callback function and a field denoting the event type.

- A MotionFrame object that contains the body-fixed frame origin, the velocity of the origin, the basis vectors and their rates of change. This provides the information needed to transform co-ordinates between the world and relative motion frames.

- Multiple Node objects, one for each float, with a unique identifier and a position field that is updated with the node's coordinates in the world frame.

- Multiple NodeMobilityController objects, one for each float, with a function handle specifying the node's actuated velocity over time. The node movement scheme is implemented in this object. 
An event is added to the priority queue whenever an up-to-date view of the network is needed or the internal state of a NodeMobilityController could change. The NetworkMobilitySimulator object removes the event from the queue having the smallest time value. The Node positions reflect the nodes' co-ordinates at the current time. For each node, its velocity in the intervening period is given by the sum of its uncontrollable velocity, given by the current velocity field, and its controllable velocity, specified by its NodeMobilityController. The node's position at the event time is found by numerically integrating the velocity function. This is achieved by calling MATLAB's ode23s [126] function, a stiff differential equation solver based on a modified Rosenbrock formula of order 2 and whose usage is described in Section 4.3.5. A stiff solver is needed as the node's actuated velocity specified by Scheme 1 is given by a series of Heaviside steps, rendering the overall velocity of the node stiff. Standard fourth-order Runge-Kutta particle-following algorithms, which are designed to solve nonstiff problems, are unsuitable in this situation as they require the step size to be taken extremely small to compute an accurate solution approximation [139]. This is repeated for each node and the body-fixed frame, and the simulation time is set to the event time. The callback function associated with the removed event is then executed, with the state of the simulator reflecting its condition just prior to the event time. The callback function may in turn add further events to the queue.

There are two main reasons for adding an event to the queue:

- To record the simulator state at the event time.

- To simulate the response of a node movement scheme to observed event: to update the internal state or actuated velocity function handle of a NodeMobilityController object.

The NetworkMobilitySimulator object continues to remove and process events until the simulation time exceeds that of the mission duration.

\section{C.3 Scheme implementation}

It has been seen that the simulator is able to model nodes' movements in a current while their actuated velocity is given by a scheme-specific velocity function, which may be updated via events. From this, a myriad of node movement strategies can be coded as subclasses of NodeMobilityController. To illustrate, the implementation of Scheme 1 is described 
At the start of the simulation, the controller inserts an event to be triggered at $t=$ $0 \mathrm{~h}$ into the priority queue with a reference to the controller's internal EventHandler method as its callback function and a string, TrajectoryGeneration, identifying the event type. The simulator is updated to the event time, and the EventHandler method is passed the TrajectoryGeneration string. The EventHandler method switches to the portion of its code dealing with trajectory generation, which accesses the Node and MotionFrame objects, reflecting the current state of the nodes and body fixed frame, and current velocity field object to find the parameters needed to specify and solve the convex optimization problem (5.36). This gives the actuated velocity at which the float needs to move to follow a cycle from the current time $t_{c}$,

$$
w_{C}(t)= \begin{cases}0 & : t<t_{c} \\ w_{C 1} & : t_{c} \leq t<t_{c}+t_{1} \\ w_{C 2} & : t_{c}+t_{1} \leq t<t_{c}+t_{1}+t_{2} \\ \cdots & \\ w_{C l} & : t_{c}+\sum_{i=1}^{l-1} t_{i} \leq t<t_{c}+\sum_{i=1}^{l} t_{i} \\ 0 & : t \geq \sum_{i=1}^{l} t_{i}\end{cases}
$$

The controller schedules another TrajectoryGeneration event after the current time, before returning execution to the NetworkMobilitySimulator object.

As the simulation proceeds, the node moves using the newly derived velocity function until the event is triggered at the end of the cycle. From the float's new position on the surface, the controls for the float's cycle in the next period are computed and the process repeated until the end of the mission.

\section{C.4 Data logging}

The output of the simulator is a record of the body-fixed frame's orientation and each node's position and velocity, both in world frame co-ordinates, at closely-spaced intervals. A PositionLoggingEvent type event is set up that, when triggered, writes the MotionFrame and Node objects to file. The state of these objects reflect their condition at the event time, $t$. In addition, the callback function schedules another PositionLoggingEvent event at a time $\Delta T$ later and a VelocityLoggingEvent to occur after $h=0.01 \mathrm{~s}$ elapses. The VelocityLoggingEvent handler reads in the past height of each node from the record and their present positions in the simulator 
to estimate their actuated speed as

$$
w_{C}(t) \approx \frac{z(t+h)-z(t)}{h}, h=0.01 \mathrm{~s} \ll 1
$$

which it writes to files as the nodes' velocities at time $t$. In our simulations, $\Delta T=$ $120 \mathrm{~s}$, resulting in 3601 records over a single run lasting 5 days. 


\section{Appendix D}

\section{Velocity properties and energy consumption of ALACE floats}

This appendix establishes how we derived our expressions for the profiling velocity limits and electrical energy consumed in mobility by ALACE floats, which are employed in Chapter 6. These expressions are original results, deduced from classical physical principles. While existing works in the literature have characterized the energy efficiency of ALACE floats [47], the usages considered were for dives to set depths, where the float remains at that depth for some time, to measure the current there, before returning back to the surface. The energy consumed by the float was thus reported in terms of the energy needed per dive or the energy needed to surface from the depth at which the profiler was established. To the best of our knowledge, we are the first to relate the energy usage to the node's velocity.

This appendix is structured as follows. The main dynamics assumptions made in our derivations are stated in Section D.1. Section D.2 presents our derivation of the ALACE float's profiling velocity limits and Section D.3 our derivation of the ALACE float's energy consumption.

\section{D.1 Model assumptions}

Our velocity and energy expressions are formulated by modelling the float as moving at the local underwater current velocity, $\mathbf{v}_{\mathrm{U}}(t)$ ( $\mathrm{U}$ for uncontrollable velocity), plus the self-propelled velocity of the float, $\mathbf{v}_{\mathrm{C}}(t)$ (c for uncontrollable velocity). The 
velocity of the float is given by

$$
\begin{aligned}
\mathbf{v}(t) & =\frac{\mathrm{d} \mathbf{x}}{\mathrm{d} t} \\
& =\mathbf{v}_{\mathrm{C}}(t)+\mathbf{v}_{\mathrm{U}}(t, \mathbf{x}) \\
& =\left[\begin{array}{lll}
0 & 0 & w_{C}(t)
\end{array}\right]^{T}+\left[\begin{array}{lll}
u_{U}(\mathbf{x}, t) & v_{U}(\mathbf{x}, t) & w_{U}(\mathbf{x}, t)
\end{array}\right]^{T}
\end{aligned}
$$

This is identical to how the profiler's velocity is specified in our node movement scheme simulation (5.11), and the same justification is used.

The vertical component of the current velocity, $w_{U}(\mathbf{x}, t)$, is considered to be able to be ignored as being negligible. This is a reasonable assumption, as the ocean is strongly stratified by density. Vertical motion is suppressed as any fluid parcel that has changed depths will be denser or lighter than the surrounding water and experience a force returning it back to its original depth. As a result, currents are mostly horizontal [32]. The velocity of the float is then given by

$$
\mathbf{v}(t)=\left[\begin{array}{lll}
u_{U}(\mathbf{x}, t) & v_{U}(\mathbf{x}, t) & w_{C}(t)
\end{array}\right]^{T}
$$

It is further assumed that the vertical velocity of the float is independent from time to time. That is to say, the float's actuation is not constrained by acceleration limits, so it can change its profiling velocity instantaneously. The temporal independence assumption is also made in $[94,130,181]$, where it is presented without justification. Upon further investigation, it was found that floats can achieve rapid velocity changes. In McGilvray and Roman [127], the development of a piston-equipped float is discussed. The paper focuses on the design of a state-space feedback control system to get the float to track a desired depth trajectory. Simulated results show that the float is able to switch between upwards and downwards velocities in $O(1 \mathrm{~s})$, as pictured in Figure D.1. The actual behaviour of floats thus closely approaches that of being able to switch instantly between velocities, our assumption.

Finally, the velocity of the float at each instant is approximated as being equal to that of the terminal velocity (D.16) set up by the drag, buoyant and weight forces balancing each other out. For this to hold true, floats must rapidly reach their terminal velocity which is another way of saying that any acceleration effects on the float are transitory. This assumption is valid for ALACE floats. In Davis and Regier [47], the depth against time profile of an ALACE float during a test mission was compared against the predicted descent of the node assuming that at each point the device is moving at terminal velocity. The two profiles are displayed in Figure D.2. The measured trajectory is marked by the solid line, while the predicted 

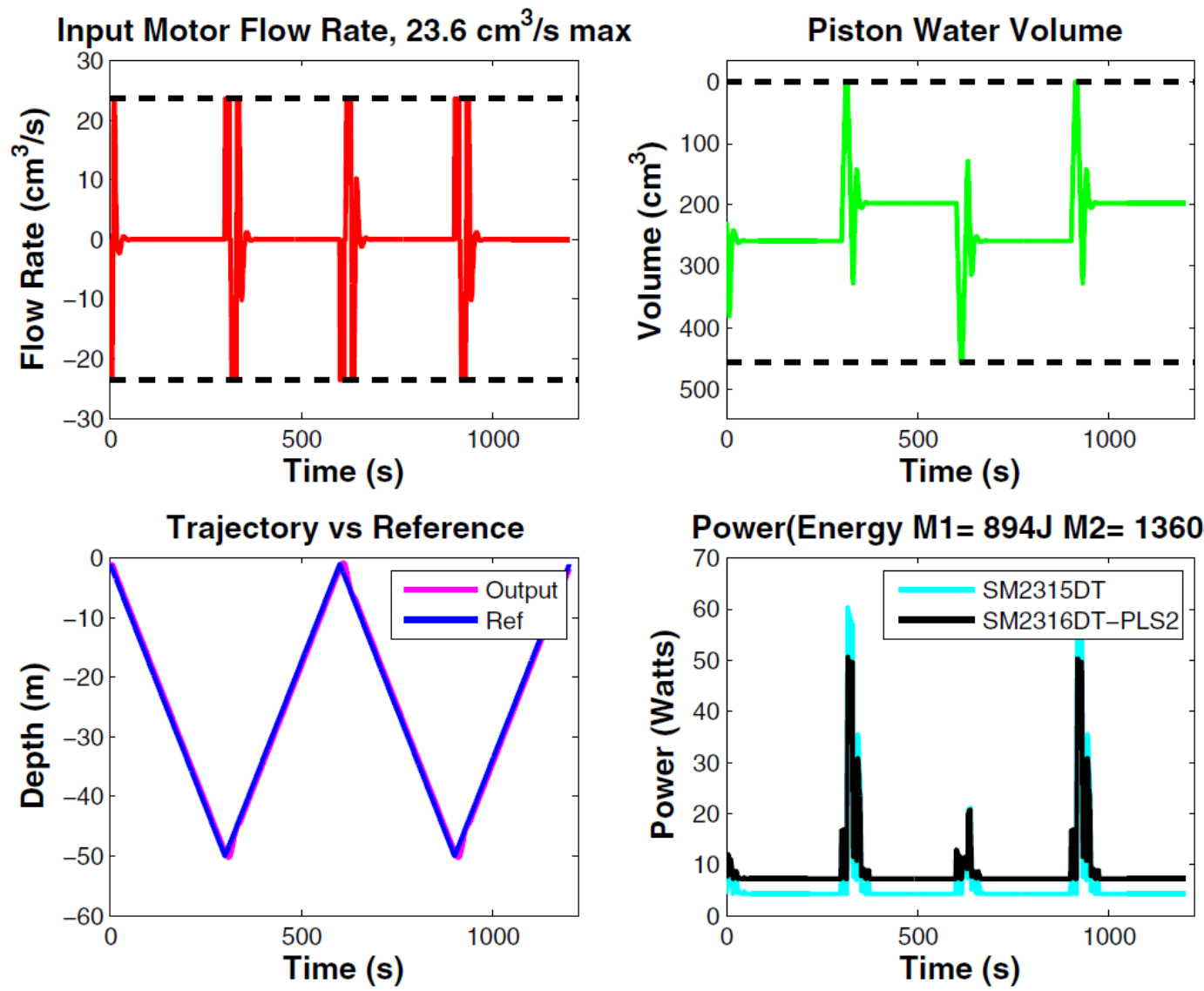

Figure D.1: Simulated response of a float, from McGilvray and Roman [127]. The blue line shows the reference profiling trajectory to be achieved, and the purple line shows the trajectory achieved by the controller. The concurrent motor, piston and power settings are also displayed. The controller is able to closely following the sawtooth trajectory, with the motor on the float being able to accelerate the instrument near instantaneously $(<1 \mathrm{~s})$ from a constant downwards velocity of $\approx 50 \mathrm{~m} / 300 \mathrm{~s}=0.1667 \mathrm{~ms}^{-1}$ to $\approx 50 \mathrm{~m} / 300 \mathrm{~s}=0.1667 \mathrm{~ms}^{-1}$ upwards.

profile is identified by the dashed line. The closeness of the two curves indicates that the actual velocity of the float is very similar to the terminal velocity. In this thesis, floats make abrupt velocity changes on the order of every 10 minutes. Given that it has been shown that floats can transition to the new constant velocity in $1 \mathrm{~s}$ of the 10 minute (see Figure D.1), it appears reasonable that the acceleration can be ignored.

\section{D.2 Velocity limits}

In our network simulation in Chapter 6 , the float is modelled as being able to move between actuated velocities $w_{C D \max } \leq w_{C}(t) \leq w_{C U \max }$ across the height range $z_{\min } \leq z \leq z_{\max }$ it operates along. In this section, expressions for the maximum 


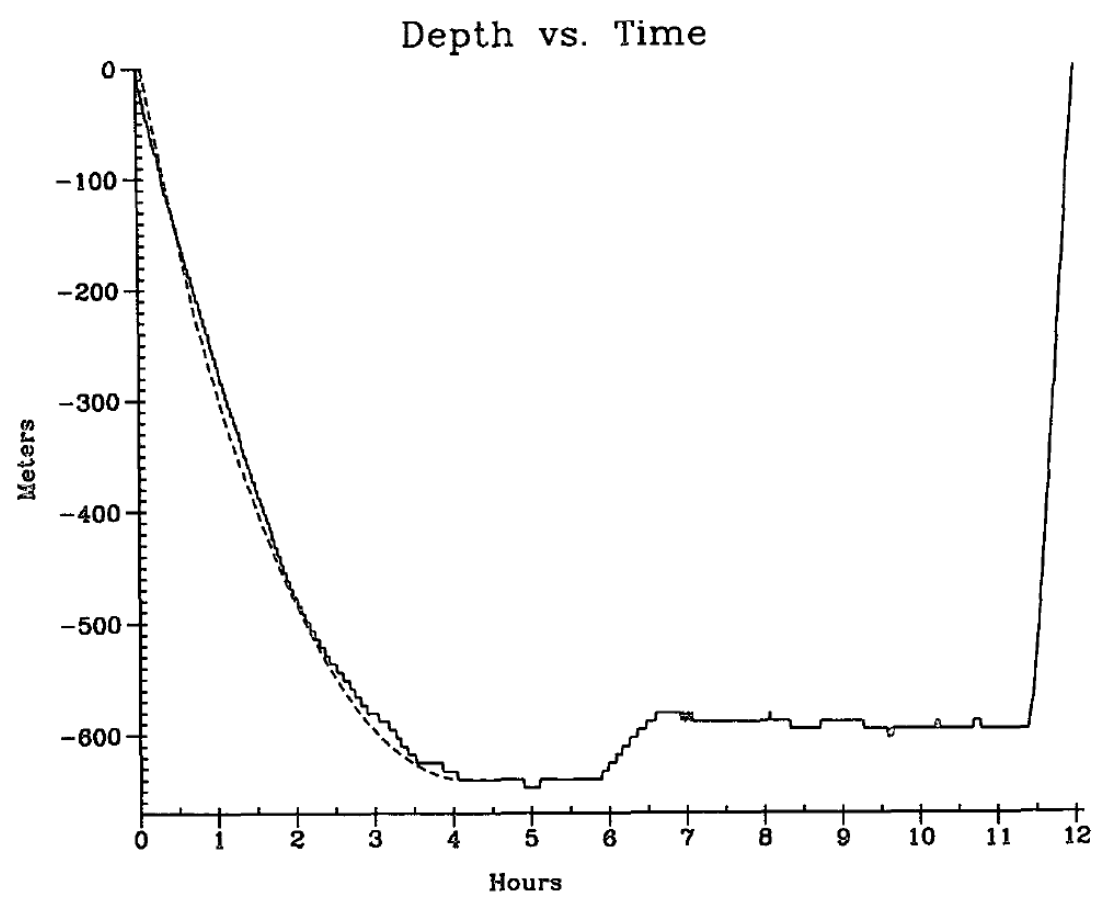

Figure D.2: Depth against time profile of an ALACE float during a test mission, from Davis and Regier [47]. The solid curve traces the measured depth of the float over time. The bladder on the float is first expanded to ballast the device to a drifting depth of $650 \mathrm{~m}$ while it is on the surface. The dashed line marks the predicted descent of the node to its initial cruising depth, assuming that the float is moving at each instant at terminal velocity. The velocity is calculated at each point using the climatological average density profile for the mission area and the measured compressibility of the float to determine the density of the water and volume displaced by the instrument, and thus the buoyant force acting on the float. The terminal velocity is then found that balances the buoyant, weight and quadratic drag forces.

achievable values of $w_{C D \max } \leq w_{C}(t) \leq w_{C U \max }$ are derived. From these relationships, realistic velocity limits can be employed in the simulations.

\section{D.2.1 Forces acting on an ALACE profiling float}

Profiling floats are scientific instruments that are used to measure underwater currents. They are able to move vertically by dynamically adjusting their buoyancy. All profiling floats possess an active buoyancy control element or volume changer (VOCHA) [150], such as an extensible piston [24,45,88,161] or inflatable external bladder [47], that allows the device to modify its volume.

Let $V_{\mathrm{F}}=V_{\mathrm{H}}+V_{\mathrm{B}}$ denote the overall volume of the float, which has been separated into the volume of its body, $V_{\mathrm{H}}$, over which the float has no control, and the volume added by the active buoyancy element, $V_{\mathrm{B}}$, which the float can vary. In the ALACE 
float, a hydraulic system is employed where the VOCHA is an external bladder protruding from the bottom of the device, as shown in Figure D.3. The bladder is filled with oil to expand it or deflated by having oil removed from it. The bladder has limited capacity where $V_{\mathrm{B}} \in\left[V_{B \min }, V_{B \max }\right]=[0,750] \mathrm{cm}^{3}[47]$.
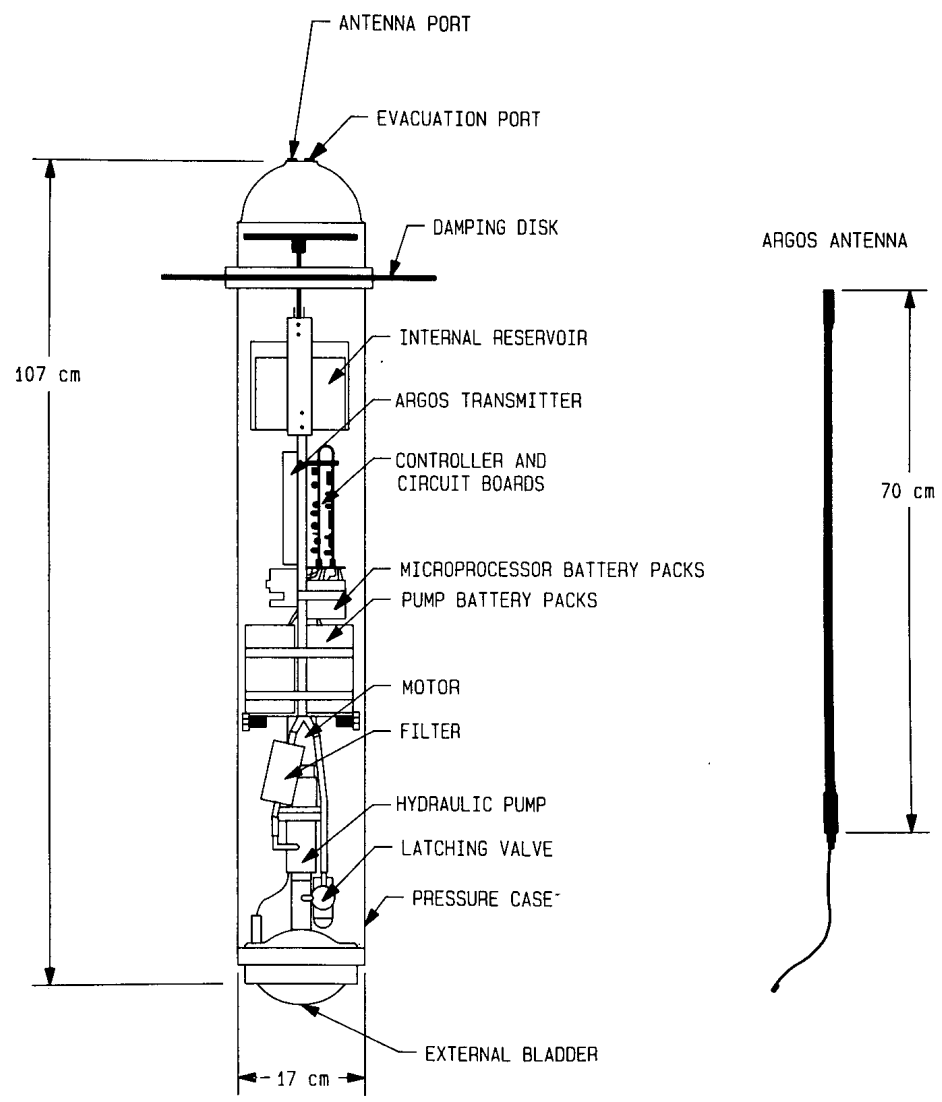

Figure D.3: Schematic of the Autonomous Lagrangian Circulation Explorer (ALACE) float, from Davis and Regier [47]. The float is housed within a cylindrical aluminium pressure case. The buoyancy control system runs the length of the float body, from the internal reservoir to the external bladder.

Figure D.4 shows the forces in the local Cartesian frame acting on the float. The downwards weight force is given by $\mathbf{F}_{\mathrm{w}}=-m g$, where $m$ is the mass of the float and $g$ is the gravity of Earth. The upwards buoyancy force is $\mathbf{F}_{\mathrm{b}}=\rho_{\mathrm{W}}(z) g\left(V_{\mathrm{H}}+V_{\mathrm{B}}\right)$, where $\rho_{\mathrm{W}}(z)$ is the density of seawater at the height above sea level $z \leq 0$ the float is located at. Archimedes' Principle states that an object displaced in a fluid will experience an upward buoyant force equal in magnitude to the weight of the fluid it displaces [173, p. 424].

Any mismatch between the two forces produces an acceleration. The motion 


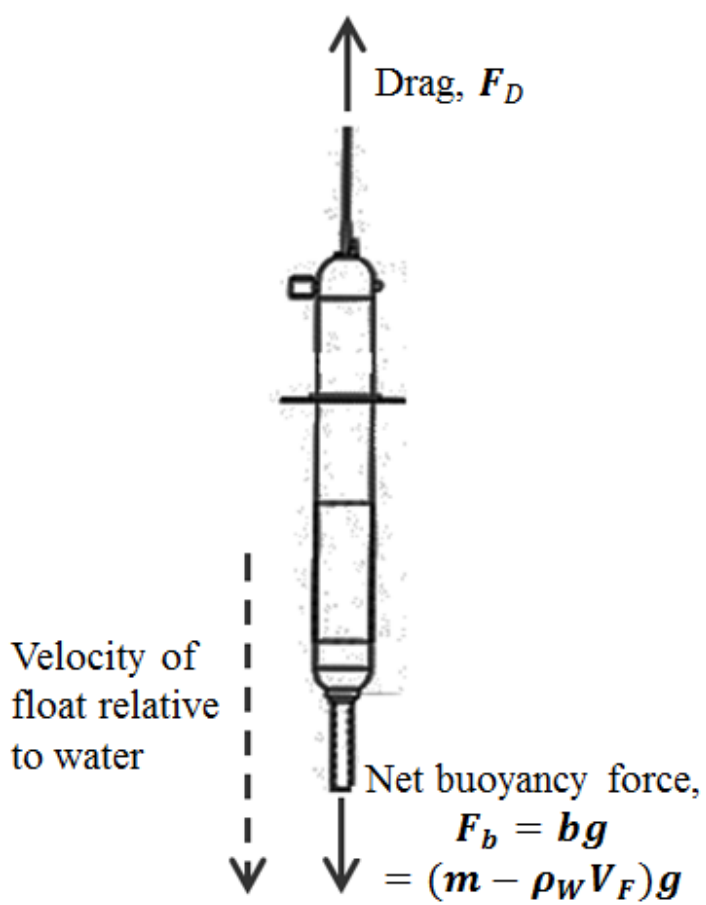

Figure D.4: Forces acting on a float as it profiles. The float experiences a downwards weight force $\mathbf{F}_{w}$ and an upwards buoyancy $\mathbf{F}_{b}$. Relative motion through the water is opposed by a drag force $\mathbf{F}_{\mathrm{D}}$.

generates an opposing resistive drag force $[45,47]$

$$
\mathbf{F}_{\mathrm{D}}=-\frac{1}{2} \rho_{\mathrm{W}}(z)\left\|\mathbf{v}_{r}\right\|^{2} C_{\mathrm{D}} A \frac{\mathbf{v}_{r}}{\left\|\mathbf{v}_{r}\right\|}
$$

where $\mathbf{v}_{r}$ is the velocity of the object relative to the water, $A$ is the area presented by the object to the motion and $C_{\mathrm{D}}$ is a dimensionless drag coefficient that depends on the shape and surface of the device. Together, $C_{\mathrm{D}} A$ is sometimes called the effective drag area of the object.

The seawater density is a function of height above the sea surface, as seawater density $\rho_{W}(z)=\rho_{W}(S, T, p)$ is influenced by three properties that vary along the water column: salinity $S$, temperature $T$ and pressure $p$. The density may be considered as monotonically increasing with depth in the open ocean [140, p. 59]. Density increases with rising salinity and pressure and falls with increasing temperature. The variation of these quantities with depth leads to the shape of the density profile. Salinity fluctuates by only a few psu (practical salinity units) and can be considered to remain fairly constant along the entire water column [146]. The temperature is highest at the surface, which is warmed by the Sun, and falls with depth. Pressure grows steadily as one descends, due to the increasing weight of the overlying water 
layers. Together, the temperature and pressure trend cause density to rise with depth. Figure D.5 shows representative open ocean profiles for density.

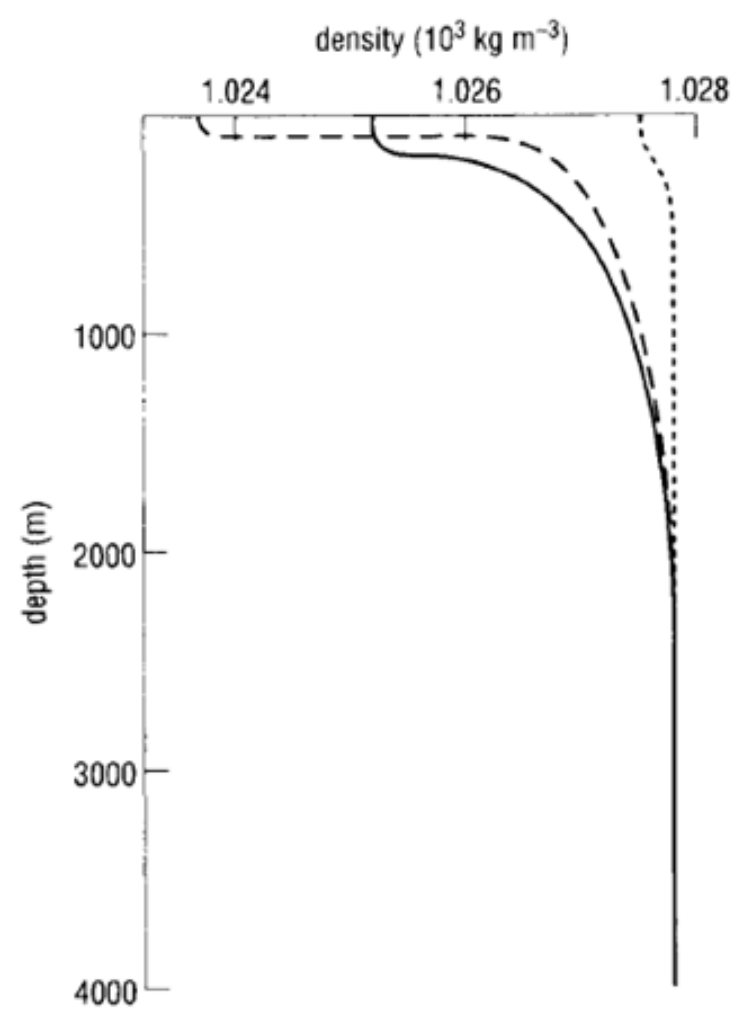

Figure D.5: Representative deep ocean density profile for different latitudes, from Brown et al [140]. The dashed line corresponds to equatorial latitudes, the solid line to tropical latitudes and the dotted line to high latitudes.

\section{D.2.2 Terminal velocity}

The total force acting on the float is

$$
\begin{aligned}
\mathbf{F} & =\mathbf{F}_{w}+\mathbf{F}_{b}+\mathbf{F}_{D} \\
& =\left(\rho_{\mathrm{W}}(z)\left(V_{\mathrm{H}}+V_{\mathrm{B}}\right)-m\right) g \hat{\mathbf{k}}-\frac{1}{2} \rho_{\mathrm{W}}(z)\left\|\mathbf{v}_{r}\right\|^{2} C_{\mathrm{D}} A \frac{\mathbf{v}_{r}}{\left\|\mathbf{v}_{r}\right\|}
\end{aligned}
$$

The float accelerates until the drag force is balanced by the buoyancy and weight forces,

$$
\mathbf{F}=\mathbf{F}_{w}+\mathbf{F}_{b}+\mathbf{F}_{D}=0
$$


The vertical velocity of the float relative to the water at this point can be found by solving (D.5) for $\mathbf{v}_{r}$,

$$
\begin{array}{r}
\left(\rho_{\mathrm{W}}(z)\left(V_{\mathrm{H}}+V_{\mathrm{B}}\right)-m\right) g \hat{\mathbf{k}}=\frac{1}{2} \rho_{\mathrm{W}}(z)\left\|\mathbf{v}_{r}\right\|^{2} C_{\mathrm{D}} A \frac{\mathbf{v}_{r}}{\left\|\mathbf{v}_{r}\right\|} \\
\mathbf{v}_{r}= \begin{cases}\sqrt{\frac{2\left[\rho_{\mathrm{W}}(z)\left(V_{\mathrm{H}}+V_{\mathrm{B}}\right)-m\right] g}{\rho_{\mathrm{W}}(z) C_{\mathrm{D}} A}} \hat{\mathbf{k}} & : \rho_{\mathrm{W}}(z)\left(V_{\mathrm{H}}+V_{\mathrm{B}}\right) \geq m \\
-\sqrt{\frac{2\left[m-\rho_{\mathrm{W}}(z)\left(V_{\mathrm{H}}+V_{\mathrm{B}}\right)\right] g}{\rho_{\mathrm{W}}(z) C_{\mathrm{D}} A}} \hat{\mathbf{k}} & : \rho_{\mathrm{W}}(z)\left(V_{\mathrm{H}}+V_{\mathrm{B}}\right)<m\end{cases}
\end{array}
$$

The velocity in (D.6) is known as the terminal velocity.

The above expression does not take into account compressibility and thermal expansion effects. The volume of the float changes as it moves between depths because it is affected by the varying temperature and pressure. The fractional change in volume of a material as the pressure changes at constant temperature is given by the substance's isothermal compressibility coefficient,

$$
\gamma=\left(\frac{1}{V} \frac{\partial V}{\partial p}\right)_{\mathrm{T}}
$$

The coefficient of thermal expansion

$$
\alpha=\left(\frac{1}{V} \frac{\partial V}{\partial T}\right)_{p}
$$

describes the material's fractional change in volume with temperature, while being held at constant pressure. Typically $\gamma>0$ and $\alpha<0$, so the volume of the float will decrease as the device descends, since temperature and pressure decrease and increase respectively monotonically with depth. Assuming constant coefficients, the volume of an object with volume $V_{b}$ in water of temperature $T_{b}$ and pressure $p_{b}$ will be $[128]$

$$
V_{m}=V_{b}\left[1+\gamma\left(p_{m}-p_{b}\right)+\alpha\left(T_{m}-T_{b}\right)\right]
$$

in water of temperature $T_{m}$ and pressure $p_{m}$.

By substituting (D.9) into the earlier float velocity equation (D.6), the actuated velocity of the float is more accurately given by

$$
w_{C}(z)= \begin{cases}\sqrt{\frac{2\left[\rho_{\mathrm{W}}(z)\left(V_{\mathrm{H}}(z)+V_{\mathrm{B}}(z)\right)-m\right] g}{\rho_{\mathrm{W}}(z) C_{\mathrm{D}} A}} & : \rho_{\mathrm{W}}(z)\left(V_{\mathrm{H}}(z)+V_{\mathrm{B}}(z)\right) \geq m \\ -\sqrt{\frac{2\left[m-\rho_{\mathrm{W}}(z)\left(V_{\mathrm{H}}(z)+V_{\mathrm{B}}(z)\right)\right] g}{\rho_{\mathrm{W}}(z) C_{\mathrm{D}} A}} & : \rho_{\mathrm{W}}(z)\left(V_{\mathrm{H}}(z)+V_{\mathrm{B}}(z)\right)<m\end{cases}
$$

where

$$
\begin{aligned}
& V_{\mathrm{H}}(z)=V_{\mathrm{H}_{0}}\left[1+\gamma_{H}\left(p-p_{0}\right)+\alpha_{H}\left(T-T_{0}\right)\right], \\
& V_{\mathrm{B}}(z)=V_{\mathrm{B}_{0}}\left[1+\gamma_{B}\left(p-p_{0}\right)+\alpha_{B}\left(T-T_{0}\right)\right]
\end{aligned}
$$


Here $\gamma_{B}, \gamma_{H}, \alpha_{B}$ and $\alpha_{H}$ are the compressibility and thermal expansion of the active buoyancy element and the body of the float respectively. The velocity being found is that of the float at sea surface height $z$. The variable $V_{H_{0}}$ is the volume of the float body on the surface, where the temperature and pressure are $T_{0}$ and $p_{0}$. The variable $V_{B_{0}}$ is the volume the VOCHA would have if the float, fixed in its current state, was moved from height $z$ to sea level.

The ALACE float's body is aluminium, with $\gamma_{H}=2.20 \times 10^{-6} \mathrm{db}^{-1}$ and $\alpha=$ $6.9 \times 10^{-5}{ }^{\circ} C^{-1}[47]$, and its VOCHA is a bladder filled with a variable quantity of hydraulic fluid, which should ideally be incompressible. The particular oil being used in the float is not given in Davis and Regier [47] but the compressibility of petroleum oils is typically very low and, as a rule of thumb, can be taken to be $\gamma=3.4 \times 10^{-8} \mathrm{db}^{-1}$ (0.5\% for each 1000 psi of pressure) [195].

The compressibility of the float body can have a significant effect on the overall volume of the ALACE float. The volume that the ALACE VOCHA can be varied between is only a small proportion of the profiler's total volume, around $3.09 \%$ for the ALACE float [47] (derived from the device dimensions given in that document). In other floats, this proportion is even less, such as $1.5 \%$ for the MLFII float [45]. The volume change due to pressure effects is often similar in order of magnitude to those affected by the active buoyancy element. For example, consider an operating depth range of $0-1000 \mathrm{~m}$. The change in volume due to pressure effects alone of the float body at $1000 \mathrm{~m}$ depth from its volume on the surface is

$$
\gamma_{H}\left(p-p_{0}\right)=2.20 \times 10^{-6} \times 1000=0.22 \%
$$

The changes to the float volume due to temperature is negligible in comparison. The typical temperature in the ocean only varies between $4-20^{\circ} \mathrm{C}$ [196], resulting in a temperature related volume change of at most

$$
\alpha_{H}\left(T-T_{0}\right)=6.9 \times 10^{-6} \times 16=0.01 \%
$$

This is much less than the $1-3 \%$ change by which the active buoyancy element can vary.

The compressibility of the VOCHA may also be ignored. The impact of pressure and temperature effects on the VOCHA is to alter the overall float volume by $\approx 0.01 \% \ll 3 \%(<1 \%$, the fractional change in the active buoyancy element's volume, of a $1 \%$, the proportion of the total float volume taken up by the VOCHA). Substituting in the compressibility of petroleum oil, over $1000 \mathrm{~m}$, the VOCHA volume, which can be no more than $1-3 \%$ of the total float volume, will change by $0.0034 \%$ from its value on the surface. 
Due to their contribution being slight, the effect of temperature and the compressibility of the VOCHA are neglected. In this thesis, the float's total volume takes into account the compressibility of the float body and the VOCHA volume, which can be actively varied, and is expressed by

$$
V_{F}=V_{\mathrm{B}}+V_{\mathrm{H}_{0}}\left[1+\gamma_{H}\left(p-p_{0}\right)\right]
$$

This results in the finalized equation for the terminal velocity of the float being

$$
\begin{aligned}
w_{C}(z) & =\sqrt{\frac{2\left[\rho_{\mathrm{W}}(z)\left(V_{\mathrm{B}}+V_{\mathrm{H}_{0}}\left[1+\gamma_{H}\left(p(z)-p_{0}\right)\right]\right)-m\right] g}{\rho_{\mathrm{W}}(z) C_{\mathrm{D}} A}} \\
& =\sqrt{\frac{2 g}{C_{\mathrm{D}} A}} \sqrt{\left(V_{\mathrm{B}}+V_{\mathrm{H}_{0}}\left[1+\gamma_{H}\left(p(z)-p_{0}\right)\right]\right)-\frac{m}{\rho_{\mathrm{W}}(z)}}
\end{aligned}
$$

where $\rho_{\mathrm{W}}(z)\left(V_{\mathrm{B}}+V_{\mathrm{H}_{0}}\left[1+\gamma_{H}\left(p(z)-p_{0}\right)\right]\right) \geq m$ and

$$
\begin{aligned}
w_{C}(z) & =-\sqrt{\frac{2\left[m-\rho_{\mathrm{W}}(z)\left(V_{\mathrm{B}}+V_{\mathrm{H}_{0}}\left[1+\gamma_{H}\left(p(z)-p_{0}\right)\right]\right)\right] g}{\rho_{\mathrm{W}}(z) C_{\mathrm{D}} A}} \\
& =-\sqrt{\frac{2 g}{C_{\mathrm{D}} A}} \sqrt{\frac{m}{\rho_{\mathrm{W}}(z)}-\left(V_{\mathrm{B}}+V_{\mathrm{H}_{0}}\left[1+\gamma_{H}\left(p(z)-p_{0}\right)\right]\right)}
\end{aligned}
$$

for $\rho_{\mathrm{W}}(z)\left(V_{\mathrm{B}}+V_{\mathrm{H}_{0}}\left[1+\gamma_{H}\left(p(z)-p_{0}\right)\right]\right)<m$.

\section{D.2.3 Velocity limits}

We derive the velocity limits of the float by first proving the following theorem.

Theorem D.2.1. The maximum upward and downwards velocity that the float can attain at a specific height are given by

$$
w_{C U}(z)=\sqrt{\frac{2 g}{C_{\mathrm{D}} A}} \sqrt{\left(V_{\mathrm{Bmax}}+V_{\mathrm{H}_{0}}\left[1+\gamma_{H}\left(p(z)-p_{0}\right)\right]\right)-\frac{m}{\rho_{\mathrm{W}}(z)}}
$$

and

$$
w_{C D}(z)=-\sqrt{\frac{2 g}{C_{\mathrm{D}} A}} \sqrt{\frac{m}{\rho_{\mathrm{W}}(z)}-\left(V_{\mathrm{Bmin}}+V_{\mathrm{H}_{0}}\left[1+\gamma_{H}\left(p(z)-p_{0}\right)\right]\right)}
$$

respectively. The first case corresponds to the VOCHA being inflated to its limit so that in (D.16) the (positive) term in the square root is made as large as possible. In the second case, the VOCHA is deflated as much as possible to minimize the term being subtracted, and so ensure that the (positive) square root value is made as large as possible. 
Since the density of seawater and pressure increase monotonically with depth, (D.16) tells us that, along the entire height range the float is constrained to operate $\left(z_{\min } \leq z \leq z_{\max }\right)$ the maximum upward actuated speed that the float can achieve at a particular value of $z$ is lowest when the device is at the height limit closest to the sea surface, $z_{\max }$. The smallest maximum downward controllable speed occurs when the float is at the deepest limit that it can move to, $z_{\min }$.

Proof. Assume that there exists a height $z_{e}<z_{\max }$ where $w_{C U}\left(z_{e}\right)<w_{C U}\left(z_{\max }\right)$. Since the other terms in (D.17) remain constant, for this to hold

$$
\begin{gathered}
\left(V_{\mathrm{Bmax}}+V_{\mathrm{H}_{0}}\left[1+\gamma_{H}\left(p\left(z_{e}\right)-p_{0}\right)\right]\right)-\frac{m}{\rho_{\mathrm{W}}\left(z_{e}\right)} \\
<\left(V_{\mathrm{Bmax}}+V_{\mathrm{H}_{0}}\left[1+\gamma_{H}\left(p\left(z_{\max }\right)-p_{0}\right)\right]\right)-\frac{m}{\rho_{\mathrm{W}}\left(z_{\max }\right)}
\end{gathered}
$$

must be true. As density and pressure increase monotonically with depth, $p\left(z_{e}\right)>$ $p\left(z_{\max }\right), \rho_{\mathrm{W}}\left(z_{e}\right)>\rho_{\mathrm{W}}\left(z_{\max }\right)$, then

$$
\begin{gathered}
\left(V_{\mathrm{Bmax}}+V_{\mathrm{H}_{0}}\left[1+\gamma_{H}\left(p\left(z_{e}\right)-p_{0}\right)\right]\right)-\frac{m}{\rho_{\mathrm{W}}\left(z_{e}\right)} \\
>\left(V_{\mathrm{Bmax}}+V_{\mathrm{H}_{0}}\left[1+\gamma_{H}\left(p\left(z_{\max }\right)-p_{0}\right)\right]\right)-\frac{m}{\rho_{\mathrm{W}}\left(z_{e}\right)} \\
>\left(V_{\mathrm{Bmax}}+V_{\mathrm{H}_{0}}\left[1+\gamma_{H}\left(p\left(z_{\max }\right)-p_{0}\right)\right]\right)-\frac{m}{\rho_{\mathrm{W}}\left(z_{\max }\right)}
\end{gathered}
$$

This contradicts (D.19) and so there is no height below $z_{\max }$ where the maximum upward speed of the float is less than $w_{C U}\left(z_{\max }\right)$, so within the operating range $z_{\min } \leq z \leq z_{\max }$

$$
\underset{z}{\arg \min } w_{C U}(z)=z_{\max }
$$

This proves the first part of the assertion.

A similar proof by contradiction is able to show that

$$
\underset{z}{\arg \max } w_{C D}(z)=z_{\min }
$$

This theorem allows us to find the maximum actuated velocities $w_{C D \max } \leq$ $w_{C}(t) \leq w_{C U \max }$ that the float can move across the height range $z_{\min } \leq z \leq z_{\max }$. These must be the smallest maximum downward and upward controllable speeds across the height range, which are given by the theorem. Otherwise, if a higher 
velocity limit was used, there will be some height within the range where the float cannot move fast enough. The velocity limits are thus given by

$$
\left[w_{C D \max }, w_{C U \max }\right]=\left[w_{C D}\left(z_{\min }\right), w_{C U}\left(z_{\max }\right)\right]
$$

where

$$
w_{C D}\left(z_{\min }\right)=-\sqrt{\frac{2 g}{C_{\mathrm{D}} A}} \sqrt{\frac{m}{\rho_{\mathrm{W}}\left(z_{\min }\right)}-\left(V_{\mathrm{Bmin}}+V_{\mathrm{H}_{0}}\left[1+\gamma_{H}\left(p\left(z_{\min }\right)-p_{0}\right)\right]\right)}
$$

and

$$
w_{C U \max }=\sqrt{\frac{2 g}{C_{\mathrm{D}} A}} \sqrt{\left(V_{\mathrm{Bmax}}+V_{\mathrm{H}_{0}}\left[1+\gamma_{H}\left(p\left(z_{\max }\right)-p_{0}\right)\right]\right)-\frac{m}{\rho_{\mathrm{W}}\left(z_{\max }\right)}}
$$

\section{D.3 Mobility electrical energy consumption}

The electrical energy consumed by an ALACE due to mobility is derived in this section.

\section{D.3.1 The energy cost of pumping}

Electrical energy is expended in mobility by ALACE floats to inflate their bladderbased VOCHAs. Figure D.6 displays a schematic of the ALACE VOCHA. To inflate the VOCHA (increasing the value of $V_{B}$ ), hydraulic oil is pumped from an internal reservoir held at $0.7 \mathrm{~atm}$ to the external bladder, which is at the pressure of the surrounding water. The amount of electrical energy depends on the efficiency of the float's pump. No energy is needed to deflate the bladder. Referring to the schematic, the latching valve is opened to reduce the latching valve is opened to lower $V_{B}$. Oil flows freely out of the bladder, at higher pressure, to the internal reservoir. Negligible electrical power is required to hold the solenoid-actuated latching valve in position [47]. It is assumed that the valve can be partially opened to achieve any desired rate of change in the VOCHA's volume.

The performance of the ALACE float pump has been characterized by Davis and Regier [47], who measured the volume of oil pumped per joule of electrical energy against pressure difference across the pump. The graph is reproduced here in Figure D.7. The efficiency has been measured with different voltages applied to the motor, with the pump speed being approximately proportional to voltage. The 


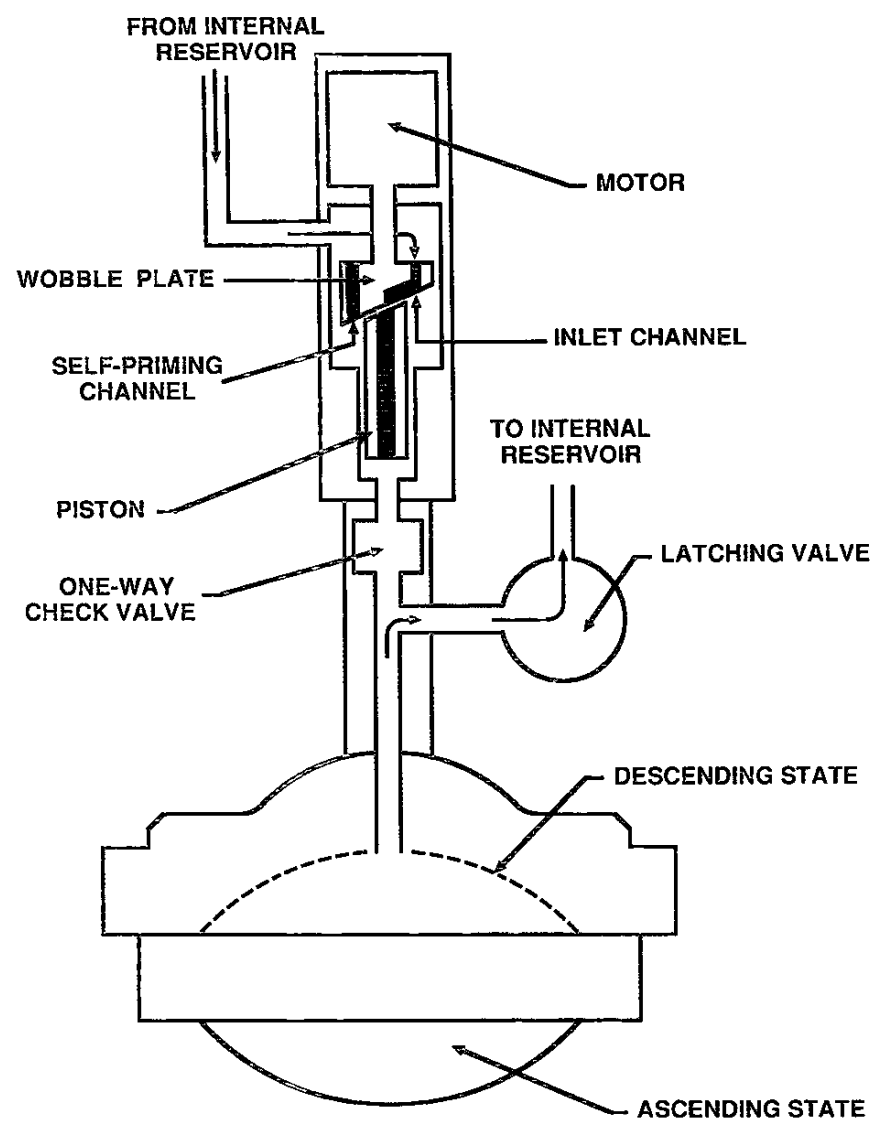

Figure D.6: Schematic of the ALACE float hydraulic system used in profiling, from Davis and Regier [47]. The volume of the float is the sum of the volume of the main float body, which is contained within its pressure case, and an external bladder that can be inflated or deflated.

value being plotted $1 / E$ is thus

$$
\begin{aligned}
d V_{B} & =\frac{1}{E\left(p, \frac{d V_{B}}{d t}\right)} d W, \\
\frac{d V_{B}}{d t}(p) & =g(p)
\end{aligned}
$$

where $W$ is the electrical energy, $p$ is the pressure difference and $g(p)$ is an unknown function, the pump speed having not been specified in Davis and Regier [47], giving the pumping rate at each pressure associated with a constant voltage.

Due to the closeness of the efficiencies among the differing supply voltages, with several values overlying each other such as the efficiencies for $12 \mathrm{~V}$ and $18 \mathrm{~V}$ at 0 dbar and $15 \mathrm{~V}$ and $18 \mathrm{~V}$ at $333 \mathrm{dbar}$, a simplification assumption is made that the efficiency is the same for all pumping rates, so

$$
d V_{B}=\frac{1}{E(z)} d W
$$




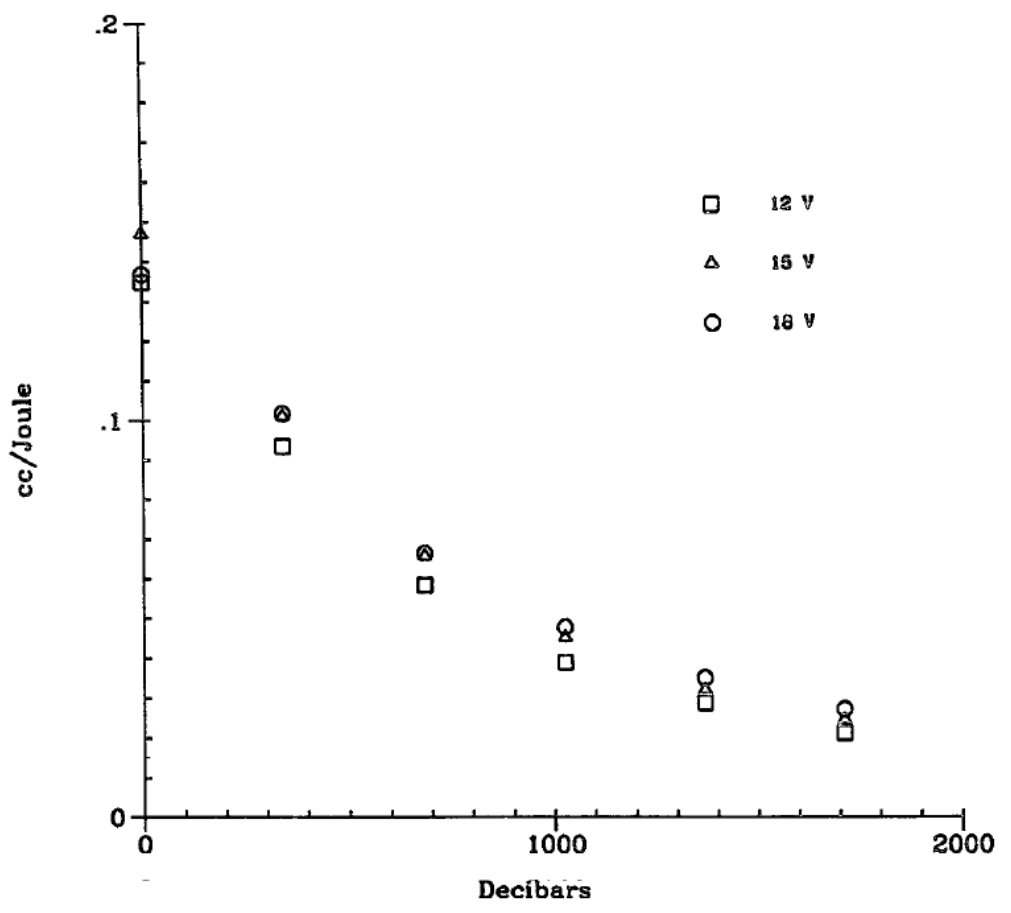

Figure D.7: ALACE float: volume of oil pumped per joule of electrical energy with various voltages applied to the motor against the pressure difference across the pump. Graph from Davis and Regier [47]

Here the pressure difference has also been converted to sea surface height:

$$
\begin{aligned}
p(z) & =10.1325-z-0.7 \times 10.1325 \\
& =3.0398-z
\end{aligned}
$$

The data points are converted so that depth is the dependant variable and presented in Figure D.8, where an exponential fit has been added. The efficiency as given by the fitted curve is

$$
\frac{1}{E(z)} \approx A e^{B z} \mathrm{~m}^{3} \mathrm{~J}^{-1}
$$

where $z$ is the height above sea level, $A=1.4507 \times 10^{-7} \mathrm{~m}^{3} \mathrm{~J}^{-1}$ and $B=0.0011 \mathrm{~m}^{-1}$.

\section{D.3.2 Electrical energy usage}

The electrical energy used by the float between times $t_{i}$ and $t_{f}$ can now be found. Every such timespan can be divided up into an alternating sequence of time intervals during which $V_{B}$ is monotonically increased and decreased

$$
t_{\mathrm{i}}=\leq t_{1 U i} \leq t_{1 U f}=t_{1 D i} \leq t_{1 D f} \ldots t_{m U i} \leq t_{m U f}=t_{p D i} \leq t_{p D f}=t_{\mathrm{f}}
$$




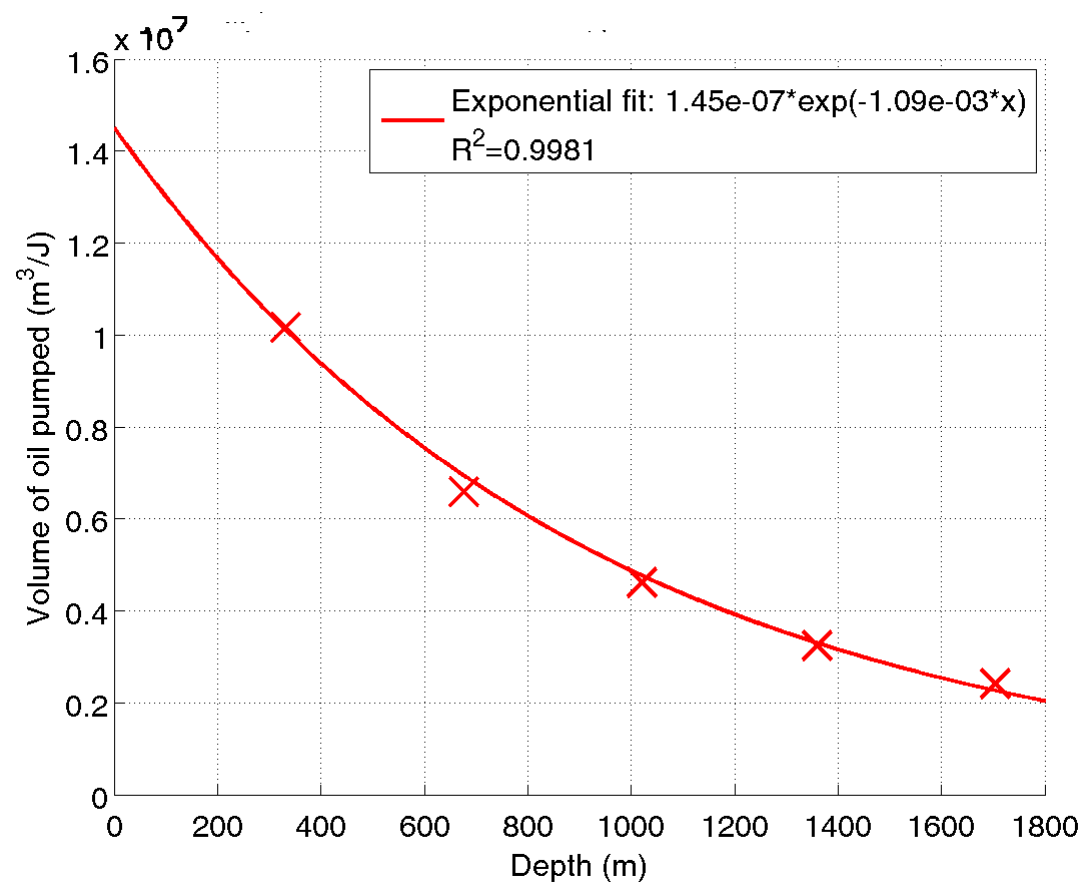

Figure D.8: ALACE float: volume of oil pumped per joule of electrical energy against depth. Graph obtained by reading data points from Davis and Regier [47]

where the subscripts $i$ and $f$ denote the start and end of each interval.

As no energy is required to deflate the VOCHA, the total energy expended by the float consists of the sum of the energy used in each interval in which the volume is monotonically increased,

$$
\begin{aligned}
& W=\int_{V_{B}\left(t_{1 U i}\right)}^{V_{B}\left(t_{1 U f}\right)} E(z(t)) \partial V_{\mathrm{B}}+\int_{V_{B}\left(t_{2 U i}\right)}^{V_{B}\left(t_{2 U f}\right)} E(z(t)) \partial V_{\mathrm{B}}+\ldots \\
& +\int_{V_{B}\left(t_{m U i}\right)}^{V_{B}\left(t_{m U f}\right)} E(z(t)) \partial V_{\mathrm{B}}
\end{aligned}
$$

where the energy used in pumping is found by integrating Equation D.27. The value of each interval can be related to the float's velocity by

$$
\begin{gathered}
W=\int_{V\left(t_{1}\right)}^{V\left(t_{2}\right)} E(z(t)) \partial V_{\mathrm{B}} \\
\stackrel{a}{=} \int_{t=t_{1}}^{t=t_{2}} E(z(t)) \frac{\partial V_{\mathrm{B}}}{\partial t} \mathrm{~d} t
\end{gathered}
$$

where (a) follows from integration by substitution, and (D.6)

$$
V_{\mathrm{B}}(t)=\frac{m}{\rho_{\mathrm{W}}(z(t))}-V_{\mathrm{H}_{0}}\left[1+\gamma_{H}\left(p(z(t))-p_{0}\right)\right]+\frac{1}{2 g}\left(w_{C}(t)\right)^{2} C_{\mathrm{D}} A \frac{w_{C}(t)}{\left\|w_{C}(t)\right\|}
$$


The total energy usage can also be written as a single definite integral of a piecewise function

$$
\begin{array}{r}
W=\int_{t=t_{1}}^{t=t_{2}} \mathcal{P}(t) d t \\
=\int_{t=t_{1}}^{t=t_{2}}\left\{\begin{array}{cc}
E(z(t)) \frac{\partial V_{\mathrm{B}}}{\partial t}, & \frac{\partial V_{\mathrm{B}}(t)}{\partial t}>0 \\
0, & \frac{\partial V_{\mathrm{B}}(t)}{\partial t} \leq 0
\end{array}\right\} d t
\end{array}
$$




\section{Appendix E}

\section{Supplementary mathematical results}

\section{E.1 Equivalence between Bower's current model and MCM}

In this section, we prove the equivalence of Bower's current mobility model [25] with the meandering current mobility model [32], by performing the necessary nondimensionalization. This allows us to use Bower's streamfunction in Chapter 3 when referring to MCM.

The current in MCM [32] is described by the non-dimensional streamfunction

$$
\begin{aligned}
\psi^{\prime}\left(x^{\prime}, y^{\prime}, t^{\prime}\right) & =-\tanh \left[\frac{y^{\prime}-B^{\prime}\left(t^{\prime}\right) \sin \left(k^{\prime}\left(x^{\prime}-c_{x}^{\prime} t^{\prime}\right)\right)}{\sqrt{1+\left(k^{\prime}\right)^{2}\left(B^{\prime}\left(t^{\prime}\right)\right)^{2} \cos ^{2}\left(k^{\prime}\left(x^{\prime}-c_{x}^{\prime} t^{\prime}\right)\right)}}\right] \\
& =-\tanh \left[\frac{y^{\prime}-\left(A^{\prime}+\epsilon^{\prime} \cos \left(\omega^{\prime} t^{\prime}\right)\right) \sin \left(k^{\prime}\left(x^{\prime}-c_{x}^{\prime} t^{\prime}\right)\right)}{\sqrt{1+\left(k^{\prime}\right)^{2}\left(A^{\prime}+\epsilon^{\prime} \cos \left(\omega^{\prime} t^{\prime}\right)\right)^{2} \cos ^{2}\left(k^{\prime}\left(x^{\prime}-c_{x}^{\prime} t^{\prime}\right)\right)}}\right]
\end{aligned}
$$

where, depending on the values that one non-dimensional unit of time $(\tau)$ and one non-dimensional unit of distance $(\xi)$ are taken to be, the physical time and spatial co-ordinates are related to their dimensionless quantities by

$$
\begin{aligned}
t^{\prime} & =t / \tau \\
x^{\prime} & =x / \xi \\
y^{\prime} & =y / \xi
\end{aligned}
$$


The streamfunction gives the current velocity in physical units as

$$
\begin{aligned}
u(x, y, t) & =-\left.\frac{\xi}{\tau} \frac{\partial \psi^{\prime}}{\partial y^{\prime}}\right|_{x^{\prime}=x / \xi, y^{\prime}=y / \xi, t^{\prime}=t / \tau} \\
v(x, y, t) & =\left.\frac{\xi}{\tau} \frac{\partial \psi^{\prime}}{\partial x^{\prime}}\right|_{x^{\prime}=x / \xi, y^{\prime}=y / \xi, t^{\prime}=t / \tau} \\
w(x, y, t) & =0
\end{aligned}
$$

In Bower's model [25], the current velocity field is defined by the dimensional streamfunction

$$
\begin{aligned}
\psi(x, y, t) & =\psi_{0}\left\{1-\tanh \left[\frac{y-y_{c}}{\lambda / \cos (\alpha)}\right]\right\} \\
& =\psi_{0}\left\{1-\tanh \left[\frac{y-B \sin \left[k\left(x-c_{x} t\right)\right]}{\lambda / \cos \left(\tan ^{-1}\left\{B k \cos \left[k\left(x-c_{x} t\right)\right]\right\}\right)}\right]\right\}
\end{aligned}
$$

that gives the current velocity as

$$
\begin{aligned}
u(x, y, t) & =-\frac{\partial \psi}{\partial y} \\
v(x, y, t) & =\frac{\partial \psi}{\partial x} \\
w(x, y, t) & =0
\end{aligned}
$$

Expanding the expression for the $u$-velocity field in Bower's model and substituting in the dimensionless quantities for space and time, $x^{\prime}=x / \xi, y^{\prime}=y / \xi$ and $t^{\prime}=t / \tau$,

$$
\begin{aligned}
u(x, y, t) & =-\frac{\partial \psi}{\partial y} \\
& =\frac{\partial}{\partial y}\left[\psi_{0}\left\{1-\tanh \left[\frac{y-B \sin \left[k\left(x-c_{x} t\right)\right]}{\lambda / \cos \left(\tan ^{-1}\left\{B k \cos \left[k\left(x-c_{x} t\right)\right]\right\}\right)}\right]\right\}\right] \\
& =\frac{\partial y^{\prime}}{\partial y} \frac{\partial}{\partial y^{\prime}}\left[\psi_{0} \tanh \left[\frac{\xi y^{\prime}-\left(A+\epsilon \cos \left(\omega \tau t^{\prime}\right)\right) \sin \left[k\left(\xi x^{\prime}-c_{x} \tau t^{\prime}\right)\right]}{\lambda / \cos \left(\tan ^{-1}\left\{\left(A+\epsilon \cos \left(\omega \tau t^{\prime}\right)\right) k \cos \left[k\left(\xi x^{\prime}-c_{x} \tau t^{\prime}\right)\right]\right\}\right)}\right]\right] \\
& =\frac{1}{\xi} \frac{\partial}{\partial y^{\prime}}\left[\psi_{0} \tanh \left[\frac{\xi y^{\prime}-\left(A+\epsilon \cos \left(\omega \tau t^{\prime}\right)\right) \sin \left[k\left(\xi x^{\prime}-c_{x} \tau t^{\prime}\right)\right]}{\lambda / \cos \left(\tan ^{-1}\left\{\left(A+\epsilon \cos \left(\omega \tau t^{\prime}\right)\right) k \cos \left[k\left(\xi x^{\prime}-c_{x} \tau t^{\prime}\right)\right]\right\}\right)}\right]\right]
\end{aligned}
$$


Now $\cos \left(\tan ^{-1}(x)\right)=1 / \sqrt{1+x^{2}}$ so

$$
\begin{aligned}
u(x, y, t) & =\frac{1}{\xi} \frac{\partial}{\partial y^{\prime}}\left[\psi_{0} \tanh \left[\frac{\xi y^{\prime}-\left(A+\epsilon \cos \left(\omega \tau t^{\prime}\right)\right) \sin \left[k\left(\xi x^{\prime}-c_{x} \tau t^{\prime}\right)\right]}{\lambda \sqrt{1+\left(A+\epsilon \cos \left(\omega \tau t^{\prime}\right)\right)^{2} k^{2} \cos ^{2}\left[k\left(\xi x^{\prime}-c_{x} \tau t^{\prime}\right)\right]}}\right]\right] \\
& =\frac{1}{\xi} \frac{\partial}{\partial y^{\prime}}\left[\psi_{0} \tanh \left[\frac{y^{\prime}-\left(\frac{A}{\xi}+\frac{\epsilon}{\xi} \cos \left(\omega \tau t^{\prime}\right)\right) \sin \left[\xi k\left(x^{\prime}-\frac{c_{x} \tau}{\xi} t^{\prime}\right)\right]}{\frac{\lambda}{\xi} \sqrt{1+\left(A+\epsilon \cos \left(\omega \tau t^{\prime}\right)\right)^{2} k^{2} \cos ^{2}\left[\xi k\left(x^{\prime}-\frac{c_{x} \tau}{\xi} t^{\prime}\right)\right]}}\right]\right] \\
& =\frac{\xi}{\tau} \frac{\partial}{\partial y^{\prime}}\left[\frac{\tau \psi_{0}}{\xi^{2}} \tanh \left[\frac{y^{\prime}-\left(\frac{A}{\xi}+\frac{\epsilon}{\xi} \cos \left(\omega \tau t^{\prime}\right)\right) \sin \left[\xi k\left(x^{\prime}-\frac{c_{x} \tau}{\xi} t^{\prime}\right)\right]}{\left.\frac{\lambda}{\xi} \sqrt{1+\left(\frac{\pi}{A} \xi+\frac{\epsilon}{\xi} \cos \left(\omega \tau t^{\prime}\right)\right)^{2}(\xi k)^{2} \cos ^{2}\left[\xi k\left(x^{\prime}-\frac{c_{x} \tau}{\xi} t^{\prime}\right)\right.}\right]}\right]\right]
\end{aligned}
$$

Defining new variables $A^{\prime \prime}=A / \xi, \epsilon^{\prime \prime}=\epsilon / \xi, \omega^{\prime \prime}=\tau \omega, k^{\prime \prime}=\tau k$ and $c_{x}^{\prime \prime}=\tau c_{x} / \xi$, we see that the $u$-velocity field is also equal to that of a MCM model, where $\xi=\lambda$ and $\tau=\lambda^{2} / \psi_{0}$

$$
\begin{aligned}
u(x, y, t) & =-\frac{\xi}{\tau} \frac{\partial}{\partial y^{\prime}}\left[-\tanh \left[\frac{y^{\prime}-\left(A^{\prime \prime}+\epsilon^{\prime \prime} \cos \left(\omega^{\prime \prime} t^{\prime}\right)\right) \sin \left[k^{\prime \prime}\left(x^{\prime}-c_{x}^{\prime \prime} t^{\prime}\right)\right]}{\sqrt{1+\left(A^{\prime \prime}+\epsilon^{\prime \prime} \cos \left(\omega^{\prime \prime} t^{\prime}\right)\right)^{2}\left(k^{\prime \prime}\right)^{2} \cos ^{2}\left[k^{\prime \prime}\left(x^{\prime}-c_{x}^{\prime \prime} t^{\prime}\right)\right]}}\right]\right] \\
& =-\left.\frac{\xi}{\tau} \frac{\partial \psi^{\prime}}{\partial y^{\prime}}\right|_{x^{\prime}=x / \xi, y^{\prime}=y / \xi, t^{\prime}=t / \tau}
\end{aligned}
$$

where $A^{\prime}=A^{\prime \prime}, \epsilon^{\prime}=\epsilon^{\prime \prime}, \omega^{\prime}=\omega^{\prime \prime}, k^{\prime \prime}=k^{\prime}, c_{x}^{\prime}=c_{x}^{\prime \prime}, \xi=\lambda$ and $\tau=\lambda^{2} / \psi_{0}$. The MCM model gives the same zonal current velocity at each point as Bower's model. Similar working can be used to show that the meridional velocities are also the same between models

$$
v(x, y, t)=\left.\frac{\xi}{\tau} \frac{\partial \psi^{\prime}}{\partial x^{\prime}}\right|_{x^{\prime}=x / \xi, y^{\prime}=y / \xi, t^{\prime}=t / \tau}
$$

In each current, the vertical velocity is zero everywhere.

$$
w(x, y, t)=0
$$

Thus, both models define equal current velocities.

\section{E.1.1 Physical significance of the MCM parameters}

We showed in Section E.1 that Bower's model expresses the same current as MCM. The relationships between the parameter settings of Bower's model and its equivalent MCM model are listed below. These can be alternatively thought of as the physical significance of the MCM parameters. 
$\xi=\lambda$, distance scaling factor.

$\tau=\lambda^{2} / \psi_{0}$, time scaling factor.

$A=\xi A^{\prime}$, average width of the meandering jet.

$\epsilon=\xi \epsilon^{\prime}$, amplitude of the modulation of the meander width.

$\omega=\omega^{\prime} / \tau$, angular frequency of the meander width amplitude modulation.

$k=k^{\prime} / \tau$, the wave number of the sinusoidal meandering jet.

$c_{x}=\xi c_{x}^{\prime} / \tau$, phase speed: the speed that the jet pattern is propagating eastward at.

\section{E.2 Density profile computation}

In this section, we describe how we computed the ocean density profile in Figure 6.3 from WOD13 depth, temperature and salinity measurements. Data from WOD13 accession number 20880 is utilized, the record of a ship sample that took measurements of the temperature and salinity at several depths at $74.55^{\circ} \mathrm{W}$ and $34.578^{\circ} \mathrm{N}$, on $23: 30$, 13 November 1970. Figure E.1 shows the geographical location of where this record was taken in the Ocean Data View utility provided for accessing the WOD data. The red cross marks the position of the selected ocean profile measurement, where each blue dot corresponds to a cast. The right "sample" panel inside the figure shows some of the physical quantities measured, which include the chlorophyll level, temperature and salinity at different depths.

We assume the ocean state throughout the simulation area is given by the recorded temperature and salinity at the single cast location. The density can then be derived using the United Nations Educational, Scientific and Cultural Organization (UNESCO) equation of state, which is accurate to $0.00035 \%$ to $10000 \mathrm{~m}$ depth [67]. The density of water with temperature $T$ (in ${ }^{\circ} C$ ), salinity $S$ (practical salinity in psu) and pressure $P$ (in bars) is given by

$$
\rho(S, T, P)=\frac{\rho(S, T, 0)}{1-P / K(S, T, P)}
$$

where $K$ is the secant bulk modulus. The values of $K$ and $\rho(S, T, 0)$ are found from 


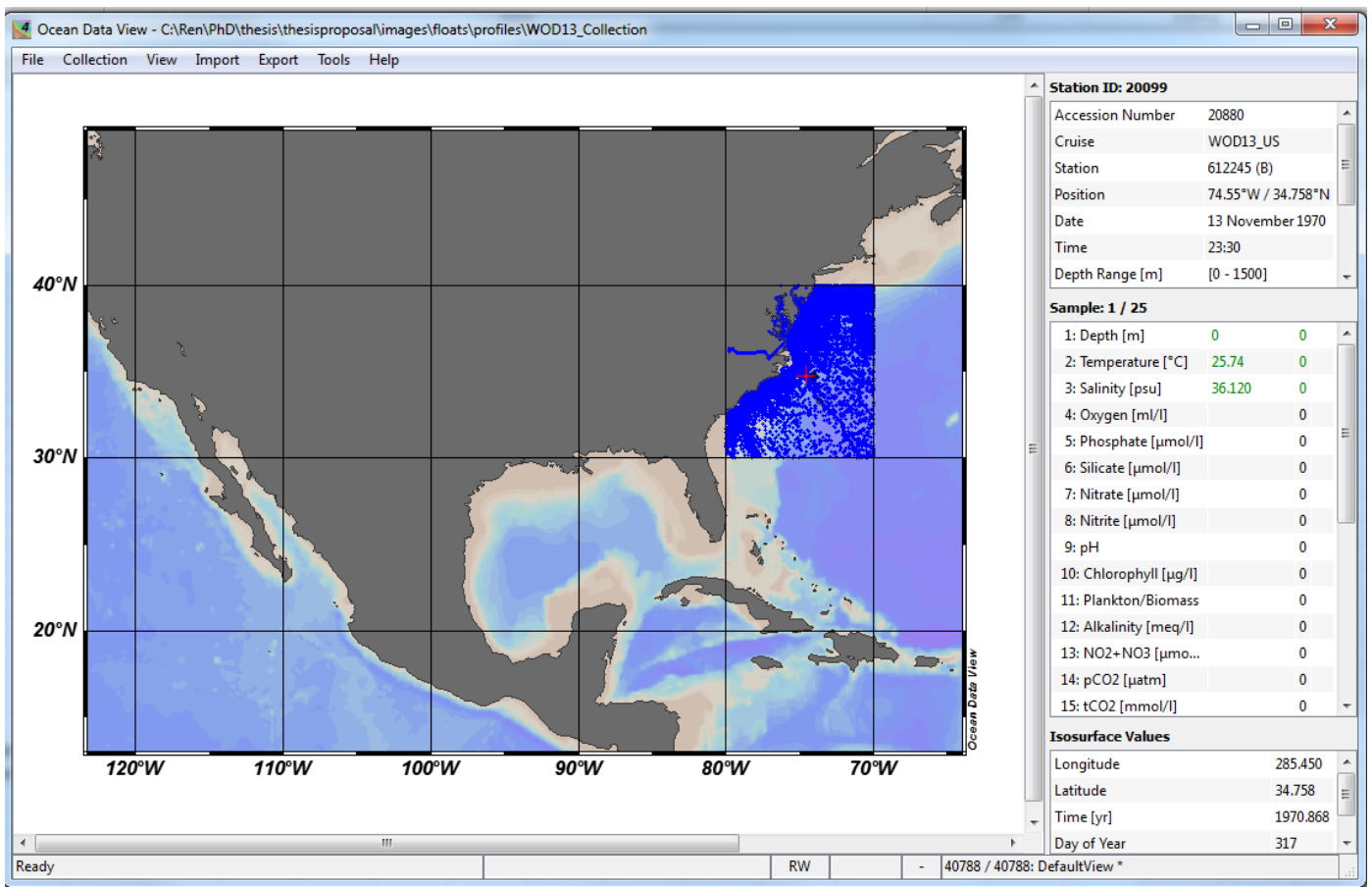

Figure E.1: Location of cast (red mark), whose ocean state samples are used to derive the density profile in 6.3 .

the density of pure water, $\rho_{\mathrm{W}}$ and the pure water secant bulk modulus, $K_{\mathrm{w}}$, where

$$
\begin{aligned}
\rho(S, T, 0) & =\rho_{\mathrm{w}} \\
& +S\left(0.824493-4.0899 \times 10^{-3} T+7.6437 \times 10^{-5} T^{2}\right. \\
& \left.-8.2467 \times 10^{-7} T^{3}+5.3875 \times 10^{-9} T^{4}\right) \\
& +S^{3 / 2}\left(-5.72466 \times 10^{-3}+1.0227 \times 10^{-4} T-1.6546 \times 10^{-6} T^{2}\right) \\
& +4.8314 \times 10^{-4} S^{2}
\end{aligned}
$$

$$
\begin{aligned}
& K(S, T, 0)=K_{\mathrm{w}}+S\left(54.6746-0.603459 T+1.09987 \times 10^{-2} T^{2}-6.1670 \times 10^{-5} T^{3}\right) \\
& \quad+S^{3 / 2}\left(7.944 \times 10^{-2}+1.6483 \times 10^{-2} T-5.3009 \times 10^{-4} T^{2}\right) \\
& K(S, T, P)=K(S, T, 0) \\
& \quad+P\left(3.239908+1.43713 \times 10^{-3} T+1.16092 \times 10^{-4} T^{2}-5.77905 \times 10^{-7} T^{3}\right) \\
& \quad+P S\left(2.2838 \times 10^{-3}-1.0981 \times 10^{-5} T-1.6078 \times 10^{-6} T^{2}\right) \\
& \quad+1.91075 \times 10^{-4} P S^{3 / 2} \\
& \quad+P^{2}\left(8.50935 \times 10^{-5}-6.12293 \times 10^{-6} T+5.2787 \times 10^{-8} T^{2}\right) \\
& \quad+P^{2} S\left(-9.9348 \times 10^{-7}+2.0816 \times 10^{-8} T+9.1697 \times 10^{-10} T^{2}\right)
\end{aligned}
$$




$$
\begin{gathered}
\rho_{\mathrm{w}}=999.842594+6.793952 \times 10^{-2} T-9.095290 \times 10^{-3} T^{2} \\
+1.001685 \times 10^{-4} T^{3}-1.120083 \times 10^{-6} T^{4}+6.536332 \times 10^{-9} T^{5} \\
K_{\mathrm{w}}=19652.21+148.4206 T-2.327105 T^{2}+1.360477 \times 10^{-2} T^{3}-5.155288 \times 10^{-5} T^{4}
\end{gathered}
$$

The pressure, which was not measured, is approximated by [140],

$$
p(z)=p_{0}-\rho_{c} g z=10.1-1.01 z
$$

where a nominal seawater density value of $\rho_{c}=1025 \mathrm{kgm}^{-3}$ is utilized and this is accurate to $< \pm 0.195 \%$ for the typical profile.

Table E.1 lists the recorded temperatures and salinities and derived pressures and densities at the corresponding depths. The cubic spline interpolated curve formed from these density values, as shown in Figure 6.3, is used for the density profile $\rho_{\mathrm{W}}(z)$ in the simulation scenario in Chapter 6 . 
Table E.1: Temperature and salinity profile of cast \#20880 taken off Cape Hatteras and derived pressure and density

\begin{tabular}{|c|c|c|c|c|}
\hline $\begin{array}{l}\text { Depth } \\
(\mathrm{m})\end{array}$ & $\begin{array}{c}\text { Temperature } \\
\left({ }^{\circ} \mathrm{C}\right)\end{array}$ & $\begin{array}{l}\text { Salinity } \\
\text { (psu) }\end{array}$ & $\begin{array}{l}\text { Pressure } \\
\text { (dbar) }\end{array}$ & $\begin{array}{r}\text { Density } \\
\left(\mathrm{kgm}^{-3}\right)\end{array}$ \\
\hline 0 & 25.74 & 36.12 & 10.132 & 1024.0 \\
\hline 10 & 25.73 & 36.13 & 20.188 & 1024.1 \\
\hline 20 & 25.73 & 36.13 & 30.243 & 1024.1 \\
\hline 30 & 25.73 & 36.13 & 40.298 & 1024.1 \\
\hline 50 & 25.71 & 36.13 & 60.409 & 1024.2 \\
\hline 75 & 24.94 & 36.14 & 85.547 & 1024.6 \\
\hline 92 & 24.83 & 36.19 & 102.641 & 1024.7 \\
\hline 100 & 24.91 & 36.41 & 110.685 & 1024.9 \\
\hline 125 & 22.82 & 36.63 & 135.823 & 1025.8 \\
\hline 150 & 20.47 & 36.52 & 160.961 & 1026.5 \\
\hline 200 & 18.64 & 36.5 & 211.237 & 1027.2 \\
\hline 250 & 18.05 & 36.5 & 261.514 & 1027.6 \\
\hline 300 & 17.78 & 36.48 & 311.790 & 1027.8 \\
\hline 400 & 17.36 & 36.45 & 412.342 & 1028.3 \\
\hline 500 & 16.29 & 36.18 & 512.895 & 1028.8 \\
\hline 600 & 14.31 & 35.84 & 613.447 & 1029.5 \\
\hline 700 & 11.97 & 35.5 & 714.000 & 1030.2 \\
\hline 800 & 9.17 & 35.17 & 814.552 & 1030.9 \\
\hline 900 & 6.81 & 35.05 & 915.105 & 1031.7 \\
\hline 1000 & 5.84 & 35.03 & 1015.657 & 1032.2 \\
\hline 1100 & 4.97 & 35.01 & 1116.210 & 1032.8 \\
\hline 1200 & 4.67 & 35.02 & 1216.762 & 1033.3 \\
\hline 1300 & 4.4 & 35.01 & 1317.315 & 1033.8 \\
\hline 1400 & 4.3 & 35.01 & 1417.868 & 1034.3 \\
\hline 1500 & 4.16 & 35.01 & 1518.420 & 1034.7 \\
\hline
\end{tabular}




\section{Bibliography}

[1] A Milestone for Ocean Observation: International Argo Program Reaches One-Millionth Profile. http://www.dfo-mpo.gc.ca/science/publications/ article/2012/12-13-12-eng.html. [Online; accessed 20-August-2015].

[2] Multi-Parametric Toolbox 3. http://www.link-quest.com/html/uwm1000. htm. [Online; last accessed 31-July-2015].

[3] Munkres' Assignment Algorithm. http://csclab.murraystate.edu/bob. pilgrim/445/munkres.html. [Online; last accessed 31-July-2015].

[4] Parallel Curve. http://xahlee.info/SpecialPlaneCurves_dir/Parallel_ dir/parallel.html. [Online; last accessed 31-July-2015].

[5] Teledyne RD Instruments Products Portal Page. http://www.rdinstruments . com/products . aspx. [Online; accessed 20-August-2015].

[6] World Ocean Database 2013. http://www.nodc.noaa.gov/OC5/WOD13/.

[7] Southern California Coastal Ocean Observing System: Surface Current Mapping. http://www.sccoos.org/data/hfrnet/, 2014. [Online; last accessed 31-July-2015].

[8] quadprog - Matlab R2015b function documentation. http://au.mathworks . com/help/optim/ug/quadprog.html, 2015. [Online; last accessed 31-July2015].

[9] Abate, R. Climate change impacts on ocean and coastal law : U.S. and international perspectives. Oxford University Press, Oxford, UK New York, NY, 2015.

[10] Akkaya, K., And Younis, M. A survey on routing protocols for wireless sensor networks. Ad Hoc Networks 3, 3 (May 2005), 325-349. 
[11] Akyildiz, I., Pompili, D., And Melodia, T. Underwater acoustic sensor networks: research challenges. Ad hoc networks 3, 3 (May 2005), 257-279.

[12] Akyildiz, I. F., And Vuran, M. C. Wireless Sensor Networks. Ian F. Akyildiz Series in Communications and Networking. John Wiley \& Sons Ltd., 2010.

[13] Alam, S., And HaAs, Z. Coverage and connectivity in three-dimensional underwater sensor networks. Wireless Communications and Mobile Computing 8, 8 (2008), 995-1009.

[14] Alvarez, A., Caiti, A., And Onken, R. Evolutionary Path Planning for Autonomous Underwater Vehicles in a Variable Ocean. IEEE Journal of Oceanic Engineering 29, 2 (Apr. 2004), 418-429.

[15] André, X., Le Reste, S., And Rolin, J.-F. Arvor-c: A coastal autonomous profiling float. Sea Technology 51, 2 (2010), 10-13.

[16] Anton, H. Elementary linear algebra. John Wiley, New York, 1994.

[17] Avis, D., Bhattacharya, B., and Imai, H. Computing the volume of the union of spheres. The Visual Computer, n 2 (1988), 323-328.

[18] Ayaz, M., Baig, I., Abdullah, A., And Faye, I. A survey on routing techniques in underwater wireless sensor networks. Journal of Network and Computer Applications 34, 6 (Nov. 2011), 1908-1927.

[19] Bayrakdar, Y., Meratnia, N., And Kantarci, A. A comparative view of routing protocols for underwater wireless sensor networks. In OCEANS, 2011 IEEE-Spain (2011), IEEE, pp. 1-5.

[20] Beerens, S., Ridderinkhof, H., And Zimmerman, J. An analytical study of chaotic stirring in tidal areas. Chaos, Solitons 86 Fractals 4, 6 (1994), 1011-1029.

[21] Bellingham, J., And Hobson, B. Future developments and applications of marine robotics. In Proceedings of the IFAC Conference (2006), MCM.

[22] Bettstetter, C., And Resta, G. The node distribution of the random waypoint mobility model for wireless ad hoc networks. Mobile Computing, IEEE 2, 3 (2003), 257-269. 
[23] Bickley, W. Lxxiii. the plane jet. The London, Edinburgh, and Dublin Philosophical Magazine and Journal of Science 23, 156 (1937), 727-731.

[24] Bokser, V., Oberg, C., Sukhatme, G., and Requicha, A. A small submarine robot for experiments in underwater sensor networks. In Symposium on Intelligent Autonomous Vehicles (2004), no. Figure 1, Citeseer, pp. 1-6.

[25] Bower, A. A simple kinematic mechanism for mixing fluid parcels across a meandering jet. Journal of Physical Oceanography (1991).

[26] Bower, A., And Rossby, T. Evidence of cross-frontal exchange processes in the Gulf Stream based on isopycnal RAFOS float data. Journal of Physical Oceanography (1989).

[27] Bower, A., And Rossby, T. Evidence of cross-frontal exchange processes in the Gulf Stream based on isopycnal RAFOS float data. Journal of Physical Oceanography (1989).

[28] Boyd, S. Convex optimization. Cambridge University Press, Cambridge, UK New York, 2004.

[29] Camilli, R., Reddy, C. M., Yoerger, D. R., Van Mooy, B. A. S., Jakuba, M. V., Kinsey, J. C., McIntyre, C. P., Sylva, S. P., And MAlONEY, J. V. Tracking hydrocarbon plume transport and biodegradation at Deepwater Horizon. Science (New York, N.Y.) 330, 6001 (Oct. 2010), 201-4.

[30] Camp, T., Boleng, J., And Davies, V. A survey of mobility models for ad hoc network research. Wireless communications and mobile computing 2, 5 (July 2002), 483-502.

[31] CAO, Y. Munkres Assignment Algorithm. http://www.mathworks.com/ matlabcentral/fileexchange/20328-munkres-assignment-algorithm, 2008. [Online; last accessed 31-July-2015].

[32] Caruso, a., Paparella, F., Vieira, L. F. M., Erol, M., and Gerla, M. The Meandering Current Mobility Model and its Impact on Underwater Mobile Sensor Networks. In 2008 IEEE INFOCOM - The 27th Conference on Computer Communications (Apr. 2008), Ieee, pp. 221-225.

[33] Cencini, M., Lacorata, G., And Vulpiani, A. Mixing in a meandering jet: a markovian approximation. Journal of Physical Oceanography, 1991 (1999), $2578-2594$. 
[34] Che, X., Wells, I., Dickers, G., Kear, P., and Gong, X. Reevaluation of RF electromagnetic communication in underwater sensor networks. Communications Magazine, IEEE 48, 12 (2010), 143-151.

[35] Cherkaoui, E., Toni, L., Rossi, L., Fontaine, J., and Agoulmine, $\mathrm{N}$. On the effects of node mobility on energy efficiency and delay sensitive applications in underwater sensor network. In OCEANS, 2011 IEEE-Spain (2011), IEEE, pp. 1-6.

[36] Chirdchoo, N., Soh, W.-S., And Chua, K. C. Sector-Based Routing with Destination Location Prediction for Underwater Mobile Networks. 2009 International Conference on Advanced Information Networking and Applications Workshops (May 2009), 1148-1153.

[37] Chiswell, S. M., And Rickard, G. J. Larval connectivity of harbours via ocean currents: A New Zealand study. Continental Shelf Research 31, 10 (July 2011), 1057-1074.

[38] Chitre, M., Shahabudeen, S., And Stojanovic, M. Underwater acoustic communications and networking: Recent advances and future challenges. Marine Technology Society Journal 42, 1 (2008), 103-116.

[39] Choset, H. Principles of Robot Motion: Theory, Algorithms, and Implementation. A Bradford book. MIT Press, 2005.

[40] Csanady, G. Circulation in the Coastal Ocean. Environmental Fluid Mechanics. Springer Netherlands, 2013.

[41] Cui, J., Kong, J., And Gerla, M. The challenges of building mobile underwater wireless networks for aquatic applications. Network, IEEE, June (2006), 12-18.

[42] Dahl, K. P., Thompson, D. R., Mclaren, D., and Chien, S. Currentsensitive path planning for an underactuated free-floating ocean Sensorweb. 2011 IEEE/RSJ International Conference on Intelligent Robots and Systems (Sept. 2011), 3140-3146.

[43] Das, J., Py, F., Maughan, T., Reilly, T. O., Messié, M., Ryan, J., Rajan, K., And Sukhatme, G. S. Simultaneous Tracking and Sampling of Dynamic Oceanographic Features with Autonomous Underwater Vehicles and Lagrangian Drifters. In Experimental Robotics, vol. 79. 2014, pp. 541-555. 
[44] D'Asaro, E., And Farmer, D. A Lagrangian float. Journal of Atmospheric ... (1996).

[45] D'Asaro, E. A. Performance of Autonomous Lagrangian Floats. Journal of Atmospheric and Oceanic Technology 20, 6 (June 2003), 896-911.

[46] D’Asaro, E. A., Farmer, D. M., Osse, J. T., and Dairiki, G. T. A lagrangian float. Journal of Atmospheric and Oceanic Technology 13, 6 (1996), $1230-1246$.

[47] Davis, R., Regier, L., Dufour, J., And Webb, D. The autonomous lagrangian circulation explorer (alace). Journal of atmospheric and oceanic technology 9, 3 (1992), 264-285.

[48] Defant, A. Quantitative Untersuchungen zur Statik und Dynamik des Atlantischen Ozeans: die absolute Topographie des physikalischen Meeresniveaus und der Druckflächen, sowie die Wasserbewegungen im Atlantischen Ozean. De Gruyter, 1941.

[49] Deif, D. S., and Gadallah, Y. Classification of wireless sensor networks deployment techniques. Communications Surveys \& Tutorials, IEEE 16, 2 (2014), 834-855.

[50] Detweiler, C., Doniec, M., Vasilescu, I., And Rus, D. Autonomous Depth Adjustment for Underwater Sensor Networks: Design and Applications. IEEE/ASME Transactions on Mechatronics 17, 1 (Feb. 2012), 16-24.

[51] Diamant, R., Wolff, L. M., and Lampe, L. Using spatial correlation of ocean current for velocity estimate of underwater drifting nodes. In Communications Workshops (ICC), 2013 IEEE International Conference on (2013), IEEE, pp. 22-26.

[52] Dietrich, I., And Dressler, F. On the lifetime of wireless sensor networks. ACM Transactions on Sensor Networks (TOSN) 5, 1 (2009), 5.

[53] Dongkyun, K., And Wahid, A. Analyzing Routing Protocols for Underwater Wireless Sensor Networks. International Journal of Communication Networks and Information Security (IJCNIS) 2, 3 (2011), 253-261.

[54] Dunbabin, M., Roberts, J., Usher, K., Winstanley, G., and Corke, P. A hybrid auv design for shallow water reef navigation. In Robotics and 
Automation, 2005. ICRA 2005. Proceedings of the 2005 IEEE International Conference on (2005), IEEE, pp. 2105-2110.

[55] Dutkiewicz, S., Griffa, A., And Olson, D. B. Particle diffusion in a meandering jet. Journal of Geophysical Research: Oceans (1978-2012) 98, C9 (1993), 16487-16500.

[56] Eidsvik, K. J. Wind-wave generation of ocean current: on model identification. Oceanic Engineering, IEEE Journal of 13, 2 (1988), 43-49.

[57] Erol, M., Vieira, L. F. M., Caruso, A., Paparella, F., Gerla, M., And OKtug, S. Multi Stage Underwater Sensor Localization Using Mobile Beacons. 2008 Second International Conference on Sensor Technologies and Applications (sensorcomm 2008) (2008), 710-714.

[58] Erol-Kantarci, M., And Mouftah, H. A Survey of Architectures and Localization Techniques for Underwater Acoustic Sensor Networks. Communications Surveys Eamp; (2011), 1-16.

[59] Erol-Kantarci, M., Oktug, S., Vieira, L., and Gerla, M. Performance evaluation of distributed localization techniques for mobile underwater acoustic sensor networks. Ad Hoc Networks 9, 1 (Jan. 2011), 61-72.

[60] Fathpour, N., Member, S., Blackmore, L., Kuwata, Y., Assad, C., Wolf, M. T., Newman, C., Elfes, A., And Reh, K. Feasibility Studies on Guidance and Global Path Planning for Wind-Assisted Montgolfière in Titan. 1112-1125.

[61] Fiorelli, E. Cooperative vehicle control, feature tracking and ocean sampling. PhD thesis, Princeton University, 2005.

[62] Fiorelli, E., Bhatta, P., Leonard, N. E., And Shulman, I. Adaptive sampling using feedback control of an autonomous underwater glider fleet. In Proc. 13th Int. Symp. on Unmanned Untethered Submersible Technology (UUST) (august 2003).

[63] Fofonoff, N., And Millard JR, R. Unesco technical papers in marine science 44. Algorithms for computation of fundamental properties of seawater (1983), 53. 
[64] Fowler, M. Viscosity. Course notes, http://galileo.phys.virginia.edu/ classes/152.mf1i.spring02/Viscosity.htm, 2007.

[65] Ghosh, A., And Das, S. K. Coverage and connectivity issues in wireless sensor networks: A survey. Pervasive and Mobile Computing 4, 3 (June 2008), 303-334.

[66] Gibson, R. Go with the flow: tidal migration in marine animals. In Migrations and Dispersal of Marine Organisms. Springer, 2003, pp. 153-161.

[67] Gill, A. Atmosphere-Ocean Dynamics. International Geophysics. Elsevier Science, 1982.

[68] Gillis, J. Giant plumes of oil forming under the Gulf. New York Times (2010), 1-4.

[69] Godfrey, M. Red algal bloom at Leigh, near Cape Rodney. http://www . niwa.co.nz/pubs/wa/13-2/images/bloom1_large.jpg/view. [Online; last accessed 31-July-2015].

[70] Guerra, F., Casari, P., And Zorzi, M. A performance comparison of MAC protocols for underwater networks using a realistic channel simulator. In OCEANS 2009, MTS/IEEE Biloxi-Marine Technology for Our Future: Global and Local Challenges (2009), IEEE, pp. 1-8.

[71] Guo, Z., Wang, B., And Cui, J. Prediction assisted singlecopy routing in underwater delay tolerant networks. In Proceedings of IEEE Globecom (2010), pp. 1-6.

[72] Halkin, D., And Rossby, T. The structure and transport of the Gulf Stream at 73 W. Journal of Physical Oceanography (1985).

[73] Hall, M. M. Horizontal and vertical structure of the gulf stream velocity field at 68 w. Journal of physical oceanography 16, 11 (1986), 1814-1828.

[74] Hall, M. M., And Bryden, H. L. Profiling the gulf stream with a current meter mooring. Geophysical research letters 12, 4 (1985), 203-206.

[75] Hallegraeff, G. M. A review of harmful algal blooms and their apparent global increase*. Phycologia 32, 2 (1993), 79-99. 
[76] Hannesson, R. Aquaculture and fisheries. Marine policy 27, 2 (2003), $169-178$.

[77] Hernández-Martínez, E. G., And Aranda-Bricaire, E. Convergence and collision avoidance in formation control: A survey of the artificial potential functions approach. INTECH Open Access Publisher, 2011.

[78] Holton, J., And Hakim, G. An Introduction to Dynamic Meteorology. Academic Press. Elsevier, 2013.

[79] Howard, A., Mataric, M., and Sukhatme, G. Mobile sensor network deployment using potential fields: A distributed, scalable solution to the area coverage problem. In Proceedings of the 6th International Symposium on Distributed Autonomous Robotics Systems (DARS02) (2002), Citeseer, pp. 299308.

[80] Howden, S. D., And Watts, D. R. Jet streaks in the gulf stream. Journal of physical oceanography 29, 8 (1999), 1910-1924.

[81] Huang, C.-F., And Tseng, Y.-C. The Coverage Problem in a Wireless Sensor Network. Mobile Networks and Applications 10, 4 (Aug. 2005), 519-528.

[82] Hutchings, J. A., And Reynolds, J. D. Marine fish population collapses: consequences for recovery and extinction risk. BioScience 54, 4 (2004), 297-309.

[83] I. E. Leonard, J. E. L. Geometry of Convex Sets, 1 ed. Wiley, 2015, p. 154.

[84] IERLEy, G. R. Boundary layers in the general ocean circulation. Annual review of fluid mechanics 22, 1 (1990), 111-140.

[85] Intanagonwiwat, C., Govindan, R., and Estrin, D. Directed diffusion: a scalable and robust communication paradigm for sensor networks. In Proceedings of the 6th annual international conference on Mobile computing and networking (2000), ACM, pp. 56-67.

[86] Iowa Department of Public Health. Harmful Algal Blooms. http: //www.idph.state.ia.us/eh/algal_blooms.asp. [Online; last accessed 31July-2015].

[87] ISLAM, M. S., AND TANAKA, M. Impacts of pollution on coastal and marine ecosystems including coastal and marine fisheries and approach for management: a review and synthesis. Marine pollution bulletin 48, 7 (2004), 624-649. 
[88] Jaffe, J., And Schurgers, C. Sensor networks of freely drifting autonomous underwater explorers. In Proceedings of the 1st ACM international workshop on Underwater networks (2006), ACM, pp. 93-96.

[89] Jamail, D. Gulf seafood deformities alarm scientists. Al Jazeera (2012).

[90] James, F. Monte carlo theory and practice. Reports on Progress in Physics 43, 9 (1980), 1145.

[91] JazAr, R. Theory of Applied Robotics: Kinematics, Dynamics, and Control. Springer, 2010.

[92] Johnson, D. B., And Maltz, D. A. Dynamic source routing in ad hoc wireless networks. In Mobile computing. Springer, 1996, pp. 153-181.

[93] Jouffroy, J., Zhou, Q., And Zielinski, O. On active current selection for lagrangian profilers. Modeling, Identification and Control (Online) 34, 1 (2013).

[94] Kämpke, T. Optimal motion paths in ambient fields. Informatica 36, 3 (2012).

[95] Kantha, L., And Clayson, C. Numerical Models of Oceans and Oceanic Processes. International Geophysics. Elsevier Science, 2000.

[96] Kantha, L., And Clayson, C. Numerical Models of Oceans and Oceanic Processes. International Geophysics. Elsevier Science, 2000.

[97] Kebkal, O., Komar, M., Kebkal, K., and Bannasch, R. D-MaC: Media access control architecture for underwater acoustic sensor networks. OCEANS 2011 IEEE - Spain (June 2011), 1-8.

[98] Kershner, R. The number of circles covering a set. American Journal of Mathematics 61, 3 (1939), 665-671.

[99] Kilfoyle, D., And A.B. Baggeroer. The state of the art in underwater acoustic telemetry. IEEE Journal of Oceanic Engineering 25, 1 (2000), 4-27.

[100] Kinsey, J. C., Eustice, R. M., And Whitcomb, L. L. A survey of underwater vehicle navigation: Recent advances and new challenges. In IFAC Conference of Manoeuvering and Control of Marine Craft (2006), vol. 88. 
[101] Klemas, V. Remote Sensing of Coastal and Ocean Currents: An Overview. Journal of Coastal Research (2012).

[102] Koenl, P., Levitt, M., And Edelsbrunner, H. ProShape Understanding the Shapes of Protein Structures. http://csb.stanford.edu/ koehl/ ProShape/overview.php, 2003. [Online; last accessed 26-August-2015].

[103] Kone, Z., Rowe, E., And Wettergren, T. Sensor repositioning to improve undersea sensor field coverage. In OCEANS (2007).

[104] Kongsberg Maritime. Data sheet - REMUS 600. http://www . km. kongsberg. com/ks/web/nokbg0397.nsf/AllWeb/ 33A681D7DB7745B4C1257A2B00286580/\$file/REMUS-600-Specs .pdf? OpenElement, 2012. [Online; accessed 20-August-2015].

[105] Korn, G. A., And Korn, T. M. Mathematical handbook for scientists and engineers: Definitions, theorems, and formulas for reference and review. Courier Corporation, 2000.

[106] Kozlov, M. K., Tarasov, S. P., and Khachiyan, L. G. The polynomial solvability of convex quadratic programming. USSR Computational Mathematics and Mathematical Physics 20, 5 (1980), 223-228.

[107] Kujawinski, E. B., Kido Soule, M. C., Valentine, D. L., Boysen, A. K., Longnecker, K., And Redmond, M. C. Fate of Dispersants Associated with the Deepwater Horizon Oil Spill. Environmental science \& technology (Jan. 2011), 1298-1306.

[108] Kuwata, Y., Blackmore, L., Wolf, M., Fathpour, N., Newman, C., AND Elfes, A. Decomposition algorithm for global reachability analysis on a time-varying graph with an application to planetary exploration. 2009 IEEE/RSJ International Conference on Intelligent Robots and Systems, IROS 2009, September (2009), 3955-3960.

[109] Kwok, A., And Martinez, S. A coverage algorithm for drifters in a river environment. In American Control Conference (ACC), 2010 (2010), IEEE, pp. 6436-6441.

[110] LaValle, S. M. Planning Algorithms. Cambridge University Press, Cambridge, U.K., 2006. Available at http://planning.cs.uiuc.edu/. 
[111] LEE, U. Personal communication.

[112] Lee, U., Kong, J., Gerla, M., Park, J., and Magistretti, E. Timecritical underwater sensor diffusion with no proactive exchanges and negligible reactive floods. Ad Hoc Networks 5, 6 (Aug. 2007), 943-958.

[113] Lee, U., Wang, P., Noh, Y., Vieira, L. F. M., Gerla, M., and Cui, J.-H. Pressure Routing for Underwater Sensor Networks. 2010 Proceedings IEEE INFOCOM (Mar. 2010), 1-9.

[114] Lemmon, C., Lui, S. M., And Lee, I. Geographic Forwarding and Routing for Ad-hoc Wireless Network: A Survey. 2009 Fifth International Joint Conference on INC, IMS and IDC (2009), 188-195.

[115] LeOnARD, N. E., AND Fiorelli, E. Virtual leaders, artificial potentials and coordinated control of groups. In Decision and Control, 2001. Proceedings of the 40th IEEE Conference on (2001), vol. 3, IEEE, pp. 2968-2973.

[116] LEwIS, E. V. Principles of naval architecture second revision. Jersey: SNAME (1988).

[117] Li, Z., Guo, Z., Hong, F., And Hong, L. E2DTS: An energy efficiency distributed time synchronization algorithm for underwater acoustic mobile sensor networks. Ad Hoc Networks (Mar. 2011).

[118] LinkQuest InC. LinkQuest Underwater Acoustic Modems UWM1000 Specifications. http://www.link-quest.com/html/uwm1000.htm. [Online; accessed 21-January-2013].

[119] Liu, B., Ren, F., Lin, C., And Yang, Y. The Redeployment Issue in Underwater Sensor Networks. IEEE GLOBECOM 2008 (2008), 1-6.

[120] Luo, J., Wang, D., And Zhang, Q. Double Mobility: Coverage of the Sea Surface with Mobile Sensor Networks. IEEE INFOCOM 2009 - The 28th Conference on Computer Communications (Apr. 2009), 118-126.

[121] Luo, J., Wang, D., And Zhang, Q. On the double mobility problem for water surface coverage with mobile sensor networks. Parallel and Distributed Systems, IEEE Transactions on 23, 1 (2012), 146-159. 
[122] Luther, M. E., Gilbert, S. A., And Tamburri, M. Status of sensors for physical oceanographic measurements. Marine Technology Society Journal 42, 1 (2008), 84-92.

[123] Mandal, A. K., Misra, S., Dash, M. K., and Ojha, T. Performance analysis of distributed underwater wireless acoustic sensor networks in the presence of internal solitons. International Journal of Communication Systems (2014).

[124] Mason, M. Mechanics of robotic manipulation. MIT Press, Cambridge, Mass, 2001.

[125] Mason, M. Mechanics of robotic manipulation. MIT Press, Cambridge, Mass, 2001, p. 101.

[126] MathWorks. Ordinary Differential Equations - Matlab R2015b function documentation. http://au.mathworks.com/help/matlab/math/ ordinary-differential-equations.html, 2015. [Online; last accessed 31July-2015].

[127] McGilvray, B., And Roman, C. Control system performance and efficiency for a mid-depth Lagrangian profiling float. OCEANS'10 IEEE Sydney, OCEANSSYD 2010 (2010).

[128] Menn, M. Instrumentation and Metrology in Oceanography. ISTE. Wiley, 2013.

[129] MIT OpenCourseWare. Kinematics of moving frames. http://ocw.mit.edu/courses/mechanical-engineering/ 2-017j-design-of-electromechanical-robotic-systems-fall-2009/ course-text/MIT2_017JF09_ch09.pdf, 2009. [Online; accessed 20-August2015].

[130] Molchanov, A., Breitenmoser, A., And Sukhatme, G. S. Active drifters: Towards a practical multi-robot system for ocean monitoring. In Robotics and Automation (ICRA), 2015 IEEE International Conference on (2015), IEEE, pp. 545-552.

[131] Neumann, G. Ocean currents, vol. 4. Elsevier, 2014. 
[132] Nicholson, J., And Healey, A. The Present State of Autonomous Underwater Vehicle (AUV) Applications and Technologies. Marine Technology Society Journal 42, 1 (2008), 44-51.

[133] Noble, M. A. Current patterns over the continental shelf and slope. Beyond the Golden Gate e Oceanography, Geology, Biology and Environment Issues in the Gulf of the Farallones. US Geological Survey Circular 1198 (2001), 67e89.

[134] Noh, Y., Lee, U., Wang, P., And Choi, B. VAPR: Void Aware Pressure Routing for Underwater Sensor Networks. IEEE Transactions on Mobile Computing (2012), 1-14.

[135] Norvig, P., And Russell, S. Artificial Intelligence: A Modern Approach. Pearson Education, 2011.

[136] NOS EDUCATION DISCOVERY KITS. Welcome to Currents Tutorial. http://oceanservice.noaa.gov/education/tutorial_currents/ lessons/currents_tutorial.pdf. [Online; accessed 20-August-2014].

[137] Oke, P., Schiller, A., Griffin, D., And Brassington, G. Ensemble data assimilation for an eddy-resolving ocean model of the australian region. Quarterly Journal of the Royal Meteorological Society 131, 613 (2005), 33013312 .

[138] Old, C., And Vennell, R. Acoustic doppler current profiler measurements of the velocity field of an ebb tidal jet. Journal of Geophysical Research: Oceans (1978-2012) 106, C4 (2001), 7037-7049.

[139] Oliver, P. J. Introduction to Partial Differential Equations. Springer, 2014, ch. Nonlinear Ordinary Differential Equations.

[140] Open University. Ocean Circulation, Second Edition, 2 ed. ButterworthHeinemann, Aug. 2001.

[141] Ott, E., And Antonsen JR, T. M. Fractal measures of passively convected vector fields and scalar gradients in chaotic fluid flows. Physical Review A 39, 7 (1989), 3660 .

[142] Paparella, F. Personal communication. 
[143] Partan, J., Kurose, J., and Levine, B. A survey of practical issues in underwater networks. ACM SIGMOBILE Mobile Computing and Communications Review 11, 4 (2007), 23-33.

[144] Paterson, A. R. A first course in fluid dynamics. Cambridge university press, 1983.

[145] Pathan, A.-S. K., And Hong, C. S. Routing in mobile ad hoc networks. In Guide to Wireless Ad Hoc Networks, S. Misra, I. Woungang, and S. Chandra Misra, Eds., Computer Communications and Networks. Springer London, London, 2009, pp. 59-96.

[146] Payne, C. M. Principles of naval weapon systems. Naval Institute Press, 2006.

[147] Perkins, C., And Royer, E. Ad-hoc on-demand distance vector routing. In Mobile Computing Systems and Applications, 1999. Proceedings. WMCSA'99. Second IEEE Workshop on (1999), IEEE, pp. 90-100.

[148] Pompili, D., Melodia, T., And Akyildiz, I. Distributed routing algorithms for underwater acoustic sensor networks. Wireless Communications, IEEE Transactions on 9, 9 (2010), 2934-2944.

[149] Pond, S. Introductory dynamical oceanography. Butterworth-Heinemann, Oxford, 1991.

[150] Prater, M. D. Eddies in the labrador sea as observed by profiling rafos floats and remote sensing. Journal of Physical Oceanography 32, 2 (2002), 411-427.

[151] Preisig, J. Acoustic propagation considerations for underwater acoustic communications network development. ACM SIGMOBILE Mobile Computing and Communications Review 11, 4 (Oct. 2007), 2.

[152] Price, J. F. Wind-driven ocean currents and Ekman transport. Deep Sea Research Part B. Oceanographic Literature Review 35, 6 (Jan. 1988), 517.

[153] Proakis, J., Sozer, E., Rice, J., And Stojanovic, M. Shallow water acoustic networks. Communications Magazine, IEEE 39, 11 (2001), 114-119.

[154] Rajagopalan, R., And Varshney, P. Data-aggregation techniques in sensor networks: a survey. IEEE Communications Surveys \& Tutorials 8, 4 (2006), 48-63. 
[155] Ravelomanana, V. Extremal properties of three-dimensional sensor networks with applications. IEEE Transactions on Mobile Computing 3, 3 (July 2004), 246-257.

[156] Reynolds, C. W. Flocks, herds and schools: A distributed behavioral model. In ACM Siggraph Computer Graphics (1987), vol. 21, ACM, pp. 25-34.

[157] Rickard, G. Personal communication.

[158] Rio, M.-H. High-frequency response of wind-driven currents measured by drifting buoys and altimetry over the world ocean. Journal of Geophysical Research 108, C8 (2003), 3283.

[159] Robinson, S. Simulation: the practice of model development and use. Palgrave Macmillan, 2014.

[160] Roemmich, D., Johnson, G. C., Riser, S., Davis, R. E., Gilson, J., Owens, W. B., Garzoli, S. L., Schmid, C., and Ignaszewski, M. The argo program: Observing the global ocean with profiling floats.

[161] Roman, C., Inglis, G., And McGilvray, B. Lagrangian floats as sea floor imaging platforms. Continental Shelf Research 31, 15 (Oct. 2011), 1592-1598.

[162] Rudin, W. Principles of Mathematical Analysis, 3rd ed. Undergraduate Texts in Mathematics. McGraw-Hill Science/Engineering/Math, 1976.

[163] Rudin, W. Principles of Mathematical Analysis, 3rd ed. Undergraduate Texts in Mathematics. McGraw-Hill Science/Engineering/Math, 1976, p. 36.

[164] Rushe, D. BP set to pay largest environmental fine in US history for Gulf oil spill. The Guardian (2015).

[165] Samelson, R. Fluid exchange across a meandering jet. Journal of physical oceanography (1992).

[166] Scalable Network Technologies, Inc. QualNet 5.0.2 Programmer's Guide. 6100 Center Drive, Suite 1250, Los Angeles, CA 90045, Mar. 2010.

[167] Schiller, A., And Smith, N. Bluelink: Large-to-coastal scale operational oceanography in the southern hemisphere. In Ocean Weather Forecasting. Springer, 2006, pp. 427-439. 
[168] Scripps Institution of Oceanography, UCSD. Scripps Scientists to Develop 'Swarms' of Miniature Robotic Ocean Explorers. https://scripps . ucsd.edu/news/2282, 2009. [Online; last accessed 31-July-2015].

[169] Seah, W. K., And Tan, H.-X. Multipath Virtual Sink Architecture for Underwater Sensor Networks. OCEANS 2006 - Asia Pacific (May 2006), 1-6.

[170] Segar, D. A., AND Segar, E. S. Introduction to ocean sciences. Wadsworth Pub., 1998.

[171] Sekula-Wood, E., Schnetzer, A., Benitez-Nelson, C. R., Anderson, C., Berelson, W. M., Brzezinski, M. A., Burns, J. M., Caron, D. A., Cetinic, I., Ferry, J. L., ET AL. Rapid downward transport of the neurotoxin domoic acid in coastal waters. Nature Geoscience 2, 4 (2009), $272-275$.

[172] Senel, F., Akkaya, K., Erol-Kantarci, M., and Yilmaz, T. Selfdeployment of mobile underwater acoustic sensor networks for maximized coverage and guaranteed connectivity. Ad Hoc Networks (Oct. 2014), 1-14.

[173] Serway, R. A., And Jewett, J. W. Physics for Scientists and Engineers with Modern Physics, 9 ed. Cengage Learning, 12013.

[174] Shaffer, G., Salinas, S., Pizarro, O., Vega, A., and Hormazabal, S. Currents in the deep ocean off chile (30 s). Deep Sea Research Part I: Oceanographic Research Papers 42, 4 (1995), 425-436.

[175] Shat, G. A. A Survey on Medium Access Control in Underwater Acoustic Sensor Networks. 2009 International Conference on Advanced Information Networking and Applications Workshops (May 2009), 1178-1183.

[176] Sharma, N., Brickley, P., Owen, G., and Coholan, P. Use of airdeployed drogued drifting buoys for oil spill tracking. Oceans $2010 \mathrm{Mts} /$ Ieee Seattle (Sept. 2010), 1-9.

[177] Shcherbina, A. Y., And Gawarkiewicz, G. G. A coastal current in winter: Autonomous underwater vehicle observations of the coastal current east of cape cod. Journal of Geophysical Research: Oceans (1978-2012) 113, C7 (2008). 
[178] Siedler, G., Church, J. A., And Gould, J. Ocean circulation and climate: observing and modelling the global ocean, vol. 77. Academic Press, 2001.

[179] Smith, R., Pereira, A., Chao, Y., And Li, P. Autonomous underwater vehicle trajectory design coupled with predictive ocean models: A case study. ICRA (2010), 4770-4777.

[180] Smith, R. N., Das, J., Chao, Y., Caron, D. A., Jones, B. H., And Sukhatme, G. S. Cooperative Multi-AUV Tracking of Phytoplankton Blooms based on Ocean Model Predictions. Physical Oceanography (2010).

[181] Smith, R. N., And Dunbabin, M. Controlled drift: An investigation into the controllability of underwater vehicles with minimal actuation. In Proceedings of the Australasian Conference on Robotics and Automation 2011 (2011), Australian Robotics \& Automation Association, pp. 1-10.

[182] Smith, R. N., And Huynh, V. T. Controlling buoyancy-driven profiling floats for applications in ocean observation. Oceanic Engineering, IEEE Journal of 39,3 (2014), 571-586.

[183] Stanway, M. J. Water profile navigation with an acoustic doppler current profiler. In OCEANS 2010 IEEE-Sydney (2010), IEEE, pp. 1-5.

[184] Steele, J. H., Thorpe, S. A., And Turekian, K. K. Measurement Techniques, Sensors and Platforms - A Derivative of Encyclopedia of Ocean Sciences (2nd Edition). Elsevier, 2009.

[185] Stewart, R. Introduction to Physical Oceanography. University Press of Florida, 2009.

[186] Stewart, R. H. The oceanic mixed layer and thermocline. http://oceanworld.tamu.edu/resources/ocng_textbook/chapter06/ chapter06_04.htm, Sept. 2006. [Online; last accessed 31-July-2015].

[187] Stojanovic, M. Underwater acoustic communication. Wiley Encyclopedia of Electrical and Electronics Engineering (1999), vol.22,pp.688-698.

[188] Swaters, G. E. On the evolution of near-singular modes of the bickley jet. Physics of Fluids (1994-present) 11, 9 (1999), 2546-2555.

[189] Syed, A. A., Heidemann, J. S., ET Al. Time synchronization for high latency acoustic networks. In INFOCOM (2006). 
[190] Tan, H.-P., Diamant, R., Seah, W. K., and Waldmeyer, M. A survey of techniques and challenges in underwater localization. Ocean Engineering 38, 14 (2011), 1663-1676.

[191] The Atlantic. The Gulf Oil Disaster: One Year Later. http://www.theatlantic.com/photo/2011/04/ the-gulf-oil-disaster-one-year-later/100049/, 2011. [Online; last accessed 31-July-2015].

[192] The Ocean Instruments Web site. AlaCe, PAlaCE and SOlO Floats. http://www . whoi.edu/instruments/viewInstrument.do?id=9706, 2014. [Online; accessed 20-August-2015].

[193] Thibodeaux, L. J., Valsaraj, K. T., John, V. T., Papadopoulos, K. D., Pratt, L. R., And Pesika, N. S. Marine Oil Fate: Knowledge Gaps, Basic Research, and Development Needs; A Perspective Based on the Deepwater Horizon Spill. Environmental Engineering Science 28, 2 (Feb. 2011), $87-93$.

[194] Thomson, W. S. On the division of space with minimum partitional area. Acta Mathematica 11, 1-4 (1887), 121-134.

[195] Totten, G. Fuels and lubricants handbook technology, properties, performance, and testing. ASTM International, West Conshohocken, PA, 2003.

[196] University of Rhode Island. Discovery of souund in the sea: How fast does sound travel? http://www.dosits.org/science/soundmovement/ speedof sound/?CFID=1342658\&CFTOKEN=55885787, 2014. [Online; accessed 20-August-2015].

[197] URICK, R. Principles of underwater sound. Peninsula Pub, Los Altos, CA, 1983.

[198] USA TODAY. Oil spill growing in the Gulf. http://usatoday30.usatoday . com/news/nation/oil-spill-map.htm, 2010. [Online; last accessed 31-July2015].

[199] VieirA, L. F. M. Underwater SEA Swarm. PhD thesis, University of California, 2009. 
[200] Wahid, A., Lee, S., And Kim, D. An energy-efficient routing protocol for UWSNs using physical distance and residual energy. In OCEANS, 2011 IEEE-Spain (2011), IEEE, pp. 1-6.

[201] Weisstein, E. W. Disk point packing. http://mathworld.wolfram.com/ DiskPointPicking.html. [Online; last accessed 31-July-2015].

[202] Whitehead, A., Dubansky, B., Bodinier, C., Garcia, T. I., Miles, S., Pilley, C., Raghunathan, V., Roach, J. L., Walker, N., Walter, R. B., ET AL. Genomic and physiological footprint of the deepwater horizon oil spill on resident marsh fishes. Proceedings of the National Academy of Sciences 109, 50 (2012), 20298-20302.

[203] Williams, M. Personal communication.

[204] Xie, P., Cui, J., And LAO, L. VBF: vector-based forwarding protocol for underwater sensor networks. Networking 2006. Networking Technologies, Services, and Protocols; Performance of Computer and Communication Networks; Mobile and Wireless Communications Systems (2006), 1216-1221.

[205] YAN, H., AND SHI, Z. DBR: depth-based routing for underwater sensor networks. Ad Hoc and Sensor Networks, Wireless Networks, (2008), 72-86.

[206] Yick, J., Mukherjee, B., And Ghosal, D. Wireless sensor network survey. Computer Networks 52, 12 (Aug. 2008), 2292-2330.

[207] Younis, M., And Akkaya, K. Strategies and techniques for node placement in wireless sensor networks: A survey. Ad Hoc Networks 6 (2008), 621-655. 